\title{
IVF culture medium and long-term child development
}

Citation for published version (APA):

Zandstra, H. (2019). IVF culture medium and long-term child development. [Doctoral Thesis, Maastricht University]. ProefschriftMaken Maastricht. https://doi.org/10.26481/dis.20190201hz

Document status and date:

Published: 01/01/2019

DOI:

10.26481/dis.20190201hz

Document Version:

Publisher's PDF, also known as Version of record

\section{Please check the document version of this publication:}

- A submitted manuscript is the version of the article upon submission and before peer-review. There can be important differences between the submitted version and the official published version of record.

People interested in the research are advised to contact the author for the final version of the publication, or visit the DOI to the publisher's website.

- The final author version and the galley proof are versions of the publication after peer review.

- The final published version features the final layout of the paper including the volume, issue and page numbers.

Link to publication

\footnotetext{
General rights rights.

- You may freely distribute the URL identifying the publication in the public portal. please follow below link for the End User Agreement:

www.umlib.nl/taverne-license

Take down policy

If you believe that this document breaches copyright please contact us at:

repository@maastrichtuniversity.nl

providing details and we will investigate your claim.
}

Copyright and moral rights for the publications made accessible in the public portal are retained by the authors and/or other copyright owners and it is a condition of accessing publications that users recognise and abide by the legal requirements associated with these

- Users may download and print one copy of any publication from the public portal for the purpose of private study or research.

- You may not further distribute the material or use it for any profit-making activity or commercial gain

If the publication is distributed under the terms of Article $25 \mathrm{fa}$ of the Dutch Copyright Act, indicated by the "Taverne" license above, 



\section{IVF CULTURE MEDIUM \\ AND LONG-TERM CHILD DEVELOPMENT}

Heleen Zandstra 
(c) H. Zandstra, Maastricht, 2018

ISBN: 978-94-6380-096-9

Cover design and layout: (c) evelienjagtman.com

Production: ProefschriftMaken || www.proefschriftmaken.nl

The research presented in this thesis was performed within GROW, School for Oncology and Developmental Biology at Maastricht University.

Performing and reporting this study was made possible by Grant No \#6- FY13-153 by March of Dimes, USA awarded to Dr. Aafke van Montfoort in 2013.

Printing of this thesis was aided by Vitrolife, Origio, Goodlife, Merck, Ferring and Birr. Their financial support was unconditional and was assigned after the thesis had been completed. 


\title{
IVF CULTURE MEDIUM \\ AND LONG-TERM CHILD DEVELOPMENT
}

\author{
PROEFSCHRIFT \\ ter verkrijging van de graad van doctor aan de Universiteit Maastricht, \\ op gezag van de Rector Magnificus, Prof. dr. Rianne M. Letschert \\ volgens het besluit van het College van Decanen, \\ in het openbaar te verdedigen op vrijdag 1 februari 2019 om 12.00 uur
}

door

Heleen Zandstra 


\section{Promotor}

Prof. dr. J.L.H. Evers

\section{Copromotores}

Dr. A.P.A. van Montfoort

Dr. J.C.M. Dumoulin

\section{Beoordelingscommissie}

Prof. dr. M.E.A. Spaanderman (voorzitter)

Prof. dr. D. Brison (University of Manchester, United Kingdom)

Prof. dr. C.D. Dirksen

Dr. W.J.M. Gerver

Prof. dr. S. Repping (Universiteit van Amsterdam) 


\section{TABLE OF CONTENTS}

Chapter 1 General introduction and outline of the thesis 7

Chapter 2 Does the type of culture medium used influence birthweight 19

of children born after IVF?

Human Reproduction, 2015 March;30(3):530-42

Chapter 3 IVF culture medium still affects weight and body composition

of child at age 9

Human Reproduction, 2018 Sep 1;33(9):1645-1656*

Chapter 4 Effect of culture medium on cardiovascular development of

9-year-old IVF children

Human Reproduction, 2018 Sep 1;33(9):1645-1656*

Chapter 5 No effect of culture medium on cognitive development of

9-year-old children

Human Reproduction Open, 2018 Sep; Issue 4, pp 1-8

Chapter 6 Growth and weight trajectories of IVF children compared to naturally conceived children

Submitted September 2018

Chapter 7 General Discussion and future prospects 133

Chapter 8 Valorisation 155

Chapter 9 English summary 163

Chapter 10 Nederlandse samenvatting 171

Chapter 11 Appendices

$\begin{array}{ll}\text { Co-authors and their affiliations } & 181\end{array}$

List of publications 183

Dankwoord 187

$\begin{array}{ll}\text { Curriculum Vitae } & 197\end{array}$

*) Chapters 3 and 4 published in combination: "Association of culture medium with growth, weight and cardiovascular development of IVF children at the age of 9 years." Human Reproduction, 2018 Sep 1;33(9):1645-1656 


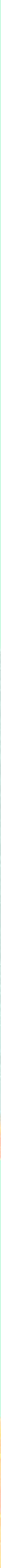




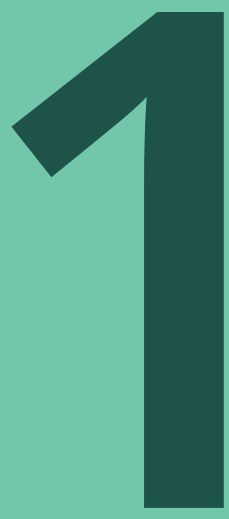

General introduction and outline of the thesis 



\section{GENERAL INTRODUCTION}

Subfertility has been described as the failure to conceive despite at least one year of regular intercourse focused on conception (Evers, 2002). It affects about $9 \%$ of all couples of reproductive age worldwide (Boivin et al., 2007). Treatment options for couples suffering from subfertility have improved over the past decades. Since the birth of the first child conceived by in vitro fertilization (IVF) in 1978 (Steptoe and Edwards, 1978), the use of assisted reproduction techniques (ART) has increased significantly. With the introduction of intracytoplasmatic sperm injection (ICSI) in 1992, this treatment could also be offered to couples dealing with severe male infertility (Palermo et al., 1992). It is estimated that around 400.000 babies are born annually through ART, worldwide (Faddy et al., 2018). After 40 years of experience with IVF/ICSI, the European Society of Human Reproduction and Embryology recently reported a total of 8 million babies conceived through this technique at its annual meeting in Barcelona (July 2018).

\section{Treatment procedures}

During an IVF treatment, the woman receives high doses of follicle stimulating hormone (FSH), to promote the development of multiple follicles within her ovaries. After an average of 14 days of FSH injections, ovulation is triggered by human Chorion Gonadotrophin (hCG) and oocytes can be collected by performing an ultrasound-guided transvaginal pick-up. Depending on the semen quality, fertilization of the oocyte will either be performed by conventional IVF (co-incubation of the oocyte and spermatozoa in a culture dish) or by ICSI (injection of a single spermatozoon directly into the oocyte). Embryos are then cultured in culture medium for several days. After 2-6 days of culture, dependent on the clinics' policy, one or more of the best quality embryos will be transferred into the uterus. Remaining embryos of good quality can be frozen and stored for later use.

\section{Effects of culture medium on IVF offspring}

Although initial research only focused on refining the techniques and improving the success rates, more recently it has come to include evaluation of perinatal and long-term health outcome of the offspring as well. In 2010, we published the first study showing that IVF culture medium might be able to induce perinatal changes in children born after IVF/ICSI (Dumoulin et al., 2010). In the period of July 2003 until December 2006, we used two commercially available culture media in an alternating way, as part of our standard care for quality control reasons. After this period, it appeared that not only there was a difference in live birth rate between the two groups, but surprisingly the children born as a result of IVF culture in Vitrolife G1, version 3 (Vitrolife AB, Göteborg, Sweden) culture medium were significantly heavier at birth than the children born after embryo culture in K-SICM (Cook Medical, Brisbane Australia) culture medium. The difference was 112 
grams after correction for possible confounders (Dumoulin et al., 2010). This was the first study to show that only a short 2-3 day period of human embryo culture can affect important perinatal outcomes like birthweight. Although not perfect as a study design, the strictly alternating use of the culture media eliminated possible confounding by changes in other culture conditions that occur over time. To analyse the observed differences in further detail, we analysed the growth during pregnancy and in the first 2 years of life. Ultrasound examinations during pregnancy showed that the growth difference between the two groups was already present at a gestational age of 20 weeks (Nelissen et al., 2013). Follow-up of these children showed that the difference was still significant at the age of 2 years (Kleijkers et al., 2014). Research on the effect of culture medium on birthweight of the IVF offspring so far is limited and not all culture media comparisons lead to the same results. The first aim of the current thesis was therefore to perform a literature review about the available evidence regarding this relationship in both human and animal.

\section{The vulnerable embryonic stage}

Comparable studies in human and animal research support the suggestion that changes during the early fetal stage may affect long-term health and development. It is well documented from epidemiological data that the nutritional status of the mother in early pregnancy may have lasting effects on the health of her offspring (Roseboom et al., 2011). Studies following the Dutch Famine during World War II showed that individuals who, as a fetus, have been exposed to the famine during early gestation, have a higher risk of adverse cardiovascular events and obesity in adult life, and show a more disadvantageous lipid profile, a disturbed blood coagulation and an increased stress response (Roseboom et al., 2006). These studies show that the fetal period is very important in determining an individual's future health.

However, animal studies have shown that, besides the fetal period, also changes in the periconceptional window can have long-term effects. It has been shown that poor nutrition solely during the periconceptional period (first 3 days after conception) followed by a normal diet during the remainder of the pregnancy can lead to increased weight, sustained hypertension and abnormal behaviour in the offspring (Watkins et al., 2011, Watkins et al., 2007). This effect was found to be induced by an altered composition of the human oviductal environment in reaction to a suboptimal diet, which is then sensed by the embryo, with long-lasting consequences. Since in vitro culture of the embryos occurs in this same (preimplantation) period, this might affect developmental programming as well (Eckert et al., 2012, Market-Velker et al., 2010). Indeed, it has been reported in mice that culture media can irreversibly affect long-term postnatal development, growth and metabolism with changes in blood pressure, glucose metabolism and altered behaviour, besides significant changes in birthweight (Velazquez et al., 2017, Watkins et al., 2007). 
Although studies on long-term effects in humans are not available, our before mentioned study showing a significant effect on birthweight induced by culture medium suggests that the same mechanisms are operative in humans (Dumoulin et al., 2010).

\section{Embryo culture medium}

The oviductal and uterine fluids surround the embryo developing in vivo on its way to implantation. Their composition is mostly determined by the maternal diet (Eckert et al., 2012). During in vitro culture, the IVF embryo relies on the culture medium for its nutrients during the first days of development. Although this short period might not appear crucial for further development, it can have long-lasting consequences since all cells within the embryo are fully exposed to the culture medium and these cells are the founding cells of all cells in the future child. The development of culture media has gone through countless steps of improvements, mostly based on animal research. In the first decade of human IVF, most embryo culture media were based on simple salt solutions supplemented with proteins, sometimes also with blood serum (Chronopoulou and Harper, 2015, Sunde et al., 2016). To improve the embryo culture, several adjustments were tested. Research focused on co-culture with fallopian tube cells and evaluating the effects of omitting or adding proteins, whole blood serum and human amniotic fluid (Chronopoulou and Harper, 2015).

Later, different approaches led to two different principles in the further improvement of the culture media; the "back-to-nature" principle and the "let-the-embryo-choose" (simplex) principle (Gardner and Leese, 1990). The "back-to-nature" principle mimics the changing needs of the developing zygote and embryo and tries to approximate the concentration of nutrients to which the embryo is naturally exposed in the female genital tract. As a foundation for the development of these culture media, reproductive fluids were studied. The recognition that the environment of the embryo changes naturally during the first few days, led to the development of sequential media which contain different ingredients during different days of culture (Gardner and Lane, 1997, Hentemann and Bertheussen, 2009). The culture media based on the "let-the-embryo-choose" principle were developed by determining the optimal concentration of each component using simplex optimization. (Chronopoulou and Harper, 2015, Lawitts and Biggers, 1991).

Nowadays, a wide variety of embryo culture media is commercially available. The compositions vary from simple solutions with "only" 8-10 different salts and sugars to media containing up to 80 different components (Chronopoulou and Harper, 2015). These usually include a mixture of salts, energy sources (e.g. glucose, lactate and pyruvate), proteins (e.g. albumin), antibiotics, amino acids, and vitamins. In the more complex media sometimes even trace ions, bioactive molecules such as hormones, growth factors and expression modulators are added. 
It is still not clear which of the two principles is preferable. It is known that the early embryo shows a more simplistic physiology with only low levels of oxidative metabolism, preferring pyruvate and lactate as primary energy sources. However as the embryo develops into a morula and reaches the compaction stage, it utilizes a wider spectrum of nutrients, the biosynthetic rates are then increasing and the embryo becomes capable of using glucose as an energy source. This shift in energy requirements coincides with the time at which the embryonic genome is activated at the post-compaction stage (Gruber and Klein, 2011).

\section{Perinatal outcome and postnatal development of children born after IVF/ICSI}

There is an increasing body of evidence showing that children born after IVF/ICSI have an increased risk of adverse perinatal outcome, such as preterm birth (PTB), low birthweight (LBW) and intrauterine growth restriction (IUGR) compared to children born after natural conception (Bergh and Wennerholm, 2012, Pinborg et al., 2013). The health outcome of the offspring and the number of congenital abnormalities is generally reassuring (Barbuscia and Mills, 2017, Hart and Norman, 2013, Hart and Norman, 2013). However, several studies have found a disadvantageous cardiovascular and metabolic outcome when IVF children were compared with naturally conceived controls. It was observed that, besides lower birthweight, IVF children also had higher systolic and diastolic blood pressure levels, higher fasting glucose levels, a higher percentage of peripheral body fat and altered endothelial function during childhood and puberty (Ceelen et al., 2009, Ceelen et al., 2007, Chen et al., 2014, Guo et al., 2017, Hart and Norman, 2013, Sakka et al., 2010, Scherrer et al., 2012). It has also been observed by some that growth of ART children lags behind that of non-ART children during the first 3 years of life (Koivurova et al., 2003). However, other studies found evidence of early catch-up growth in the first years of life with height and weight at 2-4 years being comparable to natural conception children despite a significantly lower birthweight (Lee et al., 2010, Woldringh et al., 2011, Yeung et al., 2016). Also, there is some evidence that cognitive development of IVF children is altered. In 2017, a systematic review showed a possible negative effect on cognitive development caused by fertility treatments (Rumbold et al., 2017). Most high quality studies did not find cognition differences if IVF children were compared with naturally conceived children, but some studies showed lower intelligence quotients specifically among ICSI children (Rumbold et al., 2017).

The aetiology behind the observed differences in postnatal development remains unclear and is obscured by a combination of multiple factors that might influence the outcome. It may be explained by patient factors related to their subfertile condition as such, or to technical factors involved with the treatment such as the hormonal hyperstimulation (Pontesilli et al., 2015), to culture medium composition, oxygen concentration during the culture, duration of embryo culture, the IVF/ICSI technique and freezing/thawing of the 
embryos (Henningsen and Pinborg, 2014). Especially the latter are important as, in contrast to patient factors, these offer an opportunity to improve outcome of IVF offspring. A review by Pinborg et al. (2013) demonstrated that both patient characteristics related to the subfertility as well as technical aspects involved in the ART procedure contribute to the observed differences (Pinborg et al., 2013). To which extent the separate factors are involved is still to be elucidated.

Long-term health effect of altered growth and weight

Although a difference of 112 grams in birthweight might not sound like a disturbingly large effect, we know that birthweight is an important measure, affecting future health and cardiovascular development. Barker et al. (2006) found that birthweight is strongly and inversely associated with the risk of cardiovascular diseases and diabetes at adult age (Barker, 2006). Barker and his colleagues showed that only a slight decrease in birthweight can lead to an increase in risk of cardiovascular diseases (Barker, 1999). The concept that early life factors can impact on later health has been termed the Barker hypothesis, or "the Developmental Origin of Health and Disease" (DOHaD) hypothesis. Research in this field showed that lower birthweight and poor perinatal outcome might affect postnatal growth, glucose metabolism, fat distribution and vascular function (Barker, 1999, Feuer and Rinaudo, 2012, Krishnaveni et al., 2010). Therefore, the second aim of this thesis was to analyse the effects of culture medium on long-term postnatal development of the IVF offspring. Several studies have shown that not only low birthweight per se, but the subsequent accelerated catch-up growth has long-term detrimental effects (Leunissen et al., 2009). This underlines the importance of gaining more insight in the growth trajectories of children born after ART. This led to the third aim of this thesis: to analyse whether early childhood growth velocity is associated with cardiovascular disease in IVF children. 


\section{OUTLINE OF THE THESIS}

The aims of this thesis are:

1. To review the literature on culture medium effects on birthweight;

2. To study the long-term health effects of culture medium in IVF children;

3. To analyse impact of accelerated early (infant) growth on later cardiovascular development.

The thesis focuses on culture medium effects on long-term health and development of IVF/ICSI offspring. The first aim of the thesis is to investigate whether culture medium can affect birthweight. In Chapter 2, we will present a structured literature review on the effect of embryo culture medium on birthweight of the resulting IVF offspring. The second aim of the thesis is to analyse the long-term health and development of children born after IVF/ICSI using different culture media. We will present a prospective follow-up study of children at 9 years of age who as an embryo were either cultured in K-SICM (Cook Medical, Brisbane, Australia) or Vitrolife G1, version 3 (Vitrolife, Göteborg, Sweden) culture medium. These children were asked after their ninth birthday to come to the hospital for an extensive follow-up investigation (MEDIUM-KIDS study). In Chapter 3 we will present the results of this study focusing on weight and body composition of these children to evaluate whether their difference in birthweight still persisted at 9 years of age. Since it was observed that IVF children show a more disadvantageous cardiovascular risk profile and since we know that birthweight is associated with cardiovascular health in adult life, in Chapter 4, the results on the cardiovascular development of these children will be presented. Chapter $\mathbf{5}$ will focus on the cognitive development and school performance of the children from the two culture media groups and will compare their performance with that of the average Dutch child.

Accelerated early (childhood) growth is a known risk factor for developing cardiovascular disease. To evaluate whether this was also present in the IVF children in our cohort, in Chapter 6 we analyse growth velocity in the first months of life and childhood growth velocity in relation to cardiovascular outcome and obesity in our children at 9 years of age. 


\section{REFERENCES}

Barbuscia A, Mills MC. Cognitive development in children up to age 11 years born after ART-a longitudinal cohort study. Hum Reprod 2017: 1-7.

Barker DJ. Fetal origins of cardiovascular disease. Ann Med 1999;31 Suppl 1: 3-6.

Barker DJ. Adult consequences of fetal growth restriction. Clin Obstet Gyneco/ 2006;49: 270-283.

Bergh C, Wennerholm UB. Obstetric outcome and long-term follow up of children conceived through assisted reproduction. Best Pract Res Clin Obstet Gynaecol 2012;26: 841-852.

Boivin J, Bunting L, Collins JA, Nygren KG. International estimates of infertility prevalence and treatment-seeking: potential need and demand for infertility medical care. Hum Reprod 2007;22: 1506-1512.

Ceelen M, van Weissenbruch MM, Prein J, Smit J, Vermeiden JP, Spreeuwenberg M, van Leeuwen FE, Delemarre-van de Waal HA. Growth during infancy and early childhood in relation to blood pressure and body fat measures at age 8-18 years of IVF children and spontaneously conceived controls born to subfertile parents. Hum Reprod 2009;24: 2788-2795.

Ceelen M, van Weissenbruch MM, Roos JC, Vermeiden JP, van Leeuwen FE, Delemarre-van de Waal HA. Body composition in children and adolescents born after in vitro fertilization or spontaneous conception. J Clin Endocrinol Metab 2007;92: 3417-3423.

Chen M, Wu L, Zhao J, Wu F, Davies MJ, Wittert GA, Norman RJ, Robker RL, Heilbronn LK. Altered glucose metabolism in mouse and humans conceived by IVF. Diabetes 2014;63: 3189-3198.

Chronopoulou E, Harper JC. IVF culture media: past, present and future. Hum Reprod Update 2015;21: 39-55.

Dumoulin JC, Land JA, Van Montfoort AP, Nelissen EC, Coonen E, Derhaag JG, Schreurs IL, Dunselman GA, Kester $A D$, Geraedts JP et al. Effect of in vitro culture of human embryos on birthweight of newborns. Hum Reprod 2010;25: 605-612.

Eckert J, Porter R, Watkins AJ, Burt E, Brooks S, Leese HJ, Humpherson PG, Cameron IT, Fleming TP. Metabolic induction and early responses of mouse blastocyst developmental programming following maternal low protein diet affecting life-long health. PLoS One 2012;7: e52791.

Evers JL. Female subfertility. Lancet 2002;360: 151-159.

Faddy MJ, Gosden MD, Gosden RG. A demographic projection of the contribution of assisted reproductive technologies to world population growth. Reprod Biomed Online 2018;36: 455-458.

Feuer S, Rinaudo P. Preimplantation stress and development. Birth Defects Res C Embryo Today 2012;96: 299-314.

Gardner DK, Lane M. Culture and selection of viable blastocysts: a feasible proposition for human IVF? Hum Reprod Update 1997;3: 367-382.

Gardner DK, Leese HJ. Concentrations of nutrients in mouse oviduct fluid and their effects on embryo development and metabolism in vitro. J Reprod Fertil 1990;88: 361-368.

Gruber I, Klein M. Embryo culture media for human IVF: which possibilities exist? J Turk Ger Gynecol Assoc 2011;12: 110-117.

Guo XY, Liu XM, Jin L, Wang T, Ullah K, Sheng JZ, Huang HF. Cardiovascular and metabolic profiles of offspring conceived by assisted reproductive technologies: a systematic review and meta-analysis. Fertil Steril 2017;107: 622-631 e625.

Hart R, Norman RJ. The longer-term health outcomes for children born as a result of IVF treatment: Part I--General health outcomes. Hum Reprod Update 2013;19: 232-243.

Hart R, Norman RJ. The longer-term health outcomes for children born as a result of IVF treatment. Part II--Mental health and development outcomes. Hum Reprod Update 2013;19: 244-250.

Henningsen AK, Pinborg A. Birth and perinatal outcomes and complications for babies conceived following ART. Semin Fetal Neonatal Med 2014;19: 234-238. 
Hentemann M, Bertheussen K. New media for culture to blastocyst. Fertil Steril 2009;91: 878-883.

Huang C, Li Z, Narayan KM, Williamson DF, Martorell R. Bigger babies born to women survivors of the 1959-1961 Chinese famine: a puzzle due to survival selection? J Dev Orig Health Dis 2010;1: 412-418.

Huang C, Li Z, Wang M, Martorell R. Early life exposure to the 1959-1961 Chinese famine has long-term health consequences. J Nutr 2010;140: 1874-1878.

Kleijkers SH, van Montfoort AP, Smits LJ, Viechtbauer W, Roseboom TJ, Nelissen EC, Coonen E, Derhaag JG, Bastings $L$, Schreurs IE et al. IVF culture medium affects post-natal weight in humans during the first 2 years of life. Hum Reprod 2014;29: 661-669.

Koivurova S, Hartikainen AL, Sovio U, Gissler M, Hemminki E, Jarvelin MR. Growth, psychomotor development and morbidity up to 3 years of age in children born after IVF. Hum Reprod 2003;18: 2328-2336.

Krishnaveni GV, Veena SR, Wills AK, Hill JC, Karat SC, Fall CH. Adiposity, insulin resistance and cardiovascular risk factors in 9-10-year-old Indian children: relationships with birth size and postnatal growth. J Dev Orig Health Dis 2010;1: 403-411.

Lawitts JA, Biggers JD. Optimization of mouse embryo culture media using simplex methods. J Reprod Fertil 1991;91: 543-556.

Lee SH, Lee MY, Chiang TL, Lee MS, Lee MC. Child growth from birth to 18 months old born after assisted reproductive technology--results of a national birth cohort study. Int J Nurs Stud 2010;47: 1159-1166.

Leunissen RW, Kerkhof GF, Stijnen T, Hokken-Koelega A. Timing and tempo of first-year rapid growth in relation to cardiovascular and metabolic risk profile in early adulthood. JAMA 2009;301: 2234-2242.

Market-Velker BA, Fernandes AD, Mann MR. Side-by-side comparison of five commercial media systems in a mouse model: suboptimal in vitro culture interferes with imprint maintenance. Biol Reprod 2010;83: 938-950.

Nelissen EC, Van Montfoort AP, Smits LJ, Menheere PP, Evers JL, Coonen E, Derhaag JG, Peeters LL, Coumans AB, Dumoulin JC. IVF culture medium affects human intrauterine growth as early as the second trimester of pregnancy. Hum Reprod 2013;28: 2067-2074.

Palermo G, Joris H, Devroey P, Van Steirteghem AC. Pregnancies after intracytoplasmic injection of single spermatozoon into an oocyte. Lancet 1992;340: 17-18.

Pinborg A, Wennerholm UB, Romundstad LB, Loft A, Aittomaki K, Söderström-Anttila V, Nygren KG, Hazekamp J, Bergh C. Why do singletons conceived after assisted reproduction technology have adverse perinatal outcome? Systematic review and meta-analysis. Hum Reprod Update 2013;19: 87-104.

Pontesilli M, Painter RC, Grooten IJ, van der Post JA, Mol BW, Vrijkotte TG, Repping S, Roseboom TJ. Subfertility and assisted reproduction techniques are associated with poorer cardiometabolic profiles in childhood. Reprod Biomed Online 2015;30: 258-267.

Roseboom T, de Rooij S, Painter R. The Dutch famine and its long-term consequences for adult health. Early Hum Dev 2006;82: 485-491.

Roseboom TJ, Painter RC, van Abeelen AF, Veenendaal MV, de Rooij SR. Hungry in the womb: what are the consequences? Lessons from the Dutch famine. Maturitas 2011;70: 141-145.

Rumbold AR, Moore VM, Whitrow MJ, Oswald TK, Moran LJ, Fernandez RC, Barnhart KT, Davies MJ. The impact of specific fertility treatments on cognitive development in childhood and adolescence: a systematic review. Hum Reprod 2017;32: 1489-1507.

Sakka SD, Loutradis D, Kanaka-Gantenbein C, Margeli A, Papastamataki M, Papassotiriou I, Chrousos GP. Absence of insulin resistance and low-grade inflammation despite early metabolic syndrome manifestations in children born after in vitro fertilization. Fertil Steril 2010;94: 1693-1699.

Scherrer U, Rimoldi SF, Rexhaj E, Stuber T, Duplain H, Garcin S, de Marchi SF, Nicod P, Germond M, Allemann Y et al. Systemic and pulmonary vascular dysfunction in children conceived by assisted reproductive technologies. Circulation 2012;125: 1890-1896.

Steptoe PC, Edwards RG. Birth after the reimplantation of a human embryo. Lancet 1978;2: 366. 
Sunde A, Brison D, Dumoulin J, Harper J, Lundin K, Magli MC, Van den Abbeel E, Veiga A. Time to take human embryo culture seriously. Hum Reprod 2016;31: 2174-2182.

Velazquez MA, Sheth B, Smith SJ, Eckert JJ, Osmond C, Fleming TP. Insulin and branched-chain amino acid depletion during mouse preimplantation embryo culture programmes body weight gain and raised blood pressure during early postnatal life. Biochim Biophys Acta 2017;1864: 590-600.

Watkins AJ, Lucas ES, Wilkins A, Cagampang FR, Fleming TP. Maternal periconceptional and gestational low protein diet affects mouse offspring growth, cardiovascular and adipose phenotype at 1 year of age. PLoS One 2011;6: e28745.

Watkins AJ, Platt D, Papenbrock T, Wilkins A, Eckert J, Kwong WY, Osmond C, Hanson M, Fleming TP. Mouse embryo culture induces changes in postnatal phenotype including raised systolic blood pressure. Proc Natl Acad Sci US A 2007;104: 5449-5454.

Woldringh GH, Hendriks JC, van Klingeren J, van Buuren S, Kollee LA, Zielhuis GA, Kremer JA. Weight of in vitro fertilization and intracytoplasmic sperm injection singletons in early childhood. Fertil Steril 2011;95: 2775-2777.

Yeung EH, Sundaram R, Bell EM, Druschel C, Kus C, Xie Y, Buck Louis GM. Infertility treatment and children's longitudinal growth between birth and 3 years of age. Hum Reprod 2016;31: 1621-1628. 


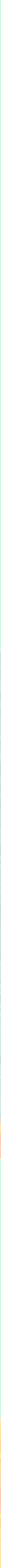




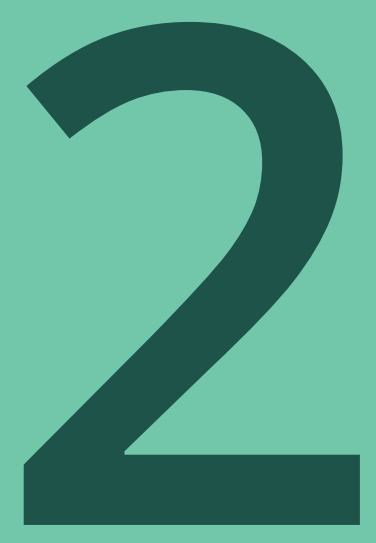

\section{Does the type of culture medium used influence birthweight of chil- dren born after IVF?}

Zandstra H, Van Montfoort APA, Dumoulin JCM

Human Reproduction, 2015 March; 30(3):530-42 


\section{ABSTRACT}

\section{Study question}

Do culture media influence birthweight of children born after IVF?

\section{Summary answer}

Some studies observed a significant effect of culture media on birthweight, while others did not but since most studies compared different culture media conventional meta-analysis was not possible.

\section{What is known already?}

Animal studies suggest that in vitro culture of embryos can have a significant effect on birthweight of offspring when compared with in vivo developed embryos. The type of culture medium (or certain components of the media) used is one of the causal factors.

\section{Study design, size, and duration}

We reviewed all available literature reporting on a relation between culture medium and birthweight in human and a selection of animal studies.

\section{Participants/materials, setting, methods}

An extensive literature search on Pubmed and Medline was performed with relevant search criteria relating to IVF, birthweight and culture medium.

\section{Main results and the role of chance}

Eleven studies reporting on a relationship between culture medium and birthweight in human were included in this review. Five of these found significant differences in birthweight when offspring born after culture in different culture media were compared. The remaining studies did not find differences in birthweight after changing culture medium.

\section{Limitations, reasons for caution}

The number of human studies is limited and different culture media with different composition are compared which makes comparison between the studies difficult if not impossible. Furthermore, most study designs were retrospective with consecutive use of different culture media and limited sample sizes, which makes bias of the results likely.

\section{Wider implications of the findings}

If it would be confirmed that the type of culture medium used does indeed influence phenotypic characteristics (such as birthweight) of children born after IVF it would underline the importance of monitoring the health of IVF children in relation with aspects of the laboratory techniques used during embryo culture. 


\section{INTRODUCTION}

There is substantial evidence that singletons born after assisted reproductive techniques (ART) are at increased risk of adverse perinatal outcome when compared to naturally conceived children. Women who conceived after ART are more likely to have a preterm ( $<37$ weeks) and very preterm birth (PTB) ( $<32$ weeks), to have their child admitted to the neonatal intensive care unit and also there is a higher risk of perinatal mortality, and congenital abnormalities (Helmerhorst et al., 2004, Jackson et al., 2004, Marino et al., 2014, McDonald et al., 2009, Pandey et al., 2012, Pinborg et al., 2013, Schieve et al., 2002). Differences in growth and development are also observed in singletons born after IVF, as they have a lower birthweight and are more likely to have a low (<2500gr) or very low birthweight (LBW) (<1500grams) and to be small for gestational age (SGA) (Helmerhorst et al., 2004, Marino et al., 2014, Pandey et al., 2012, Schieve et al., 2002). The Developmental Origins of Health and Disease (DOHaD) hypothesis underlines the relevance of evaluating the effects of ART on growth and development of IVF children, as it is described that a lower birth weight in non-IVF children is associated with an increased risk for a number of adult diseases (Barker, 2007, Gillman et al., 2007, Godfrey et al., 2010). Although it is too early to state that the LBW after IVF will have the same consequences, the DOHaD theory shows that changes in the early developmental environment can have long-lasting effects, which obligates researchers to explore these effects in the IVF offspring (Bloise et al., 2014).

The exact mechanisms behind the observed perinatal differences are still to be elucidated. Several factors associated with ART have been suggested to be involved, including maternal factors associated with infertility. It has been shown in many studies that subfertility itself is associated with adverse outcome. In a study conducted by Romundstad et al. (2008) comparing pregnancy outcomes from 2546 women who had at least one child after ART and a second child after spontaneous conception, no differences in perinatal outcome of ART versus non-ART siblings were found suggesting that parental factors rather than the ART technique are responsible for the adverse outcomes reported in ART offspring (Romundstad et al., 2008). Also in a meta-analysis of 14 studies it was concluded that children conceived after spontaneous conception from subfertile women are associated with a moderate increase in the risk of LBW and SGA (Messerlian et al., 2013). These results were confirmed in a large nationwide perinatal registry studies from Australia (Marino et al., 2014). 
However there is growing evidence that IVF procedures (ovarian stimulation, ovum pick up and embryo transfer) and culture (medium) have a share in the observed differences as well. A systematic review of sibling-studies comparing ART children with their naturally conceived sibling found, in contrast to the above mentioned study by Romundstad et al. (2008), that ART children have a higher risk of preterm birth than their non-ART siblings (Pinborg et al., 2013). These results could indicate that ART-procedures at least partly explain the differences in perinatal outcome. Several authors explored the relationship between ovarian hyperstimulation and the perinatal differences (Imudia et al., 2013, Nakashima et al., 2013, Pelinck et al., 2010). Pelinck et al. (2010) found that birthweight was not altered by stimulation protocol and Griesinger et al. (2008) also found that dose of FSH and days of stimulation was not related to birthweight. Nakashima et al. (2013) however showed in a regression analysis that birthweight was significantly correlated with the stimulation protocol after comparison of outcome after modified natural cycle IVF, clomiphene citrate and conventional IVF with different stimulation protocols. The influence of ART technology on perinatal characteristics such as birthweight is clearly demonstrated in studies that compare children born after fresh transfer with those of children born after cryopreservation. In these comparisons, subfertility is ruled out as determining factor as children from both groups are born from the same population of subfertile women. Several sibling- and large cohort studies showed that singletons born after FET have a higher birthweight and that the chances of having LBW, very PTB and SGA are reduced when compared to singletons born after fresh embryo transfer (Henningsen et al., 2011, Marino et al., 2014, Nakashima et al., 2013, Pelinck et al., 2010, Wennerholm et al., 2013). Although it is described that parity can influence birthweight, sibling comparisons corrected for order of birth showed that the differences in birthweight remain after correction (Henningsen et al., 2011). The observed differences between outcome after FET compared to fresh transfer are evident, however the question which scenario is the most favorable is yet to be answered. For example, some studies report a higher risk for large for gestational age (LGA) and macrosomia in the cryopreservation group (Pinborg et al., 2014, Sazonova et al., 2012, Wennerholm et al., 2013). It is however unclear from these studies which aspects of the ART technology are responsible for the observed differences. It could be speculated that either the minimal hormonal stimulation that is often used in FET cycles or aspects related to the cryopreservation technique could be involved. The theory that cryopreservation might be responsible is supported by results after fresh transfer in oocyte donation cycles where the uterine environment is identical to FET cycles. In several studies, even a higher risk for LBW and PTB was observed, also after correction for the higher prevalence of preeclampsia (Gibbons et al., 2011, Malchau et al., 2013, Zegers-Hochschild et al., 2010). 
Recently, more attention was given to the effect of culture medium on perinatal outcomes, especially since the publications from our group, where we found a relationship between culture medium and birthweight and altered growth patterns starting as early as the second trimester of pregnancy and even being present at two years of age (Dumoulin et al., 2010, Kleijkers et al., 2014, Nelissen et al., 2012, Nelissen et al., 2013). Several studies with conflicting results on this topic have been published since then. The aim of this review is to review available literature on the influence of culture (medium) on birthweight and growth of IVF offspring in human studies and also discuss a selection of animal studies with a special focus on culture medium effects. Answering the question whether or not there is a true relationship between culture conditions and perinatal outcome is extremely relevant because elucidating these mechanisms could be helpful in reducing perinatal risks such as LBW. 


\section{MATERIALS AND METHODS}

For the present review, we identified articles eligible for this review through extensive searches on Pubmed and Medline using the following medical subject headings (MESH) and text words: ((IVF or ART or "in vitro fertilization" or "in vitro fertilization" or "in-vitro fertilization" or "in-vitro fertilisation" or "assisted reproductive technique" or "assisted reproductive technic" or ICSI or "Intracytoplasmic sperm injection" or "embryo transfer") and (birthweight or "birth weight") and ("culture medium" or "culture media" or "embryo culture media" or "embryo culture medium" or "culture-media" or "culture-medium")). The result was checked by hand search of references of included articles and personal literature collections of the authors. We included all articles reporting on a relationship between culture medium and human birthweight and written in English published before 15 October 2014. We also present a selection of animal studies reporting on effect of culture media on birthweight. As birthweight was a side analysis in most animal studies, most were not traceable with the above mentioned search strategy. Therefore we do not pretend to give an exhaustive enumeration of all studies but present all relevant papers to the best of our knowledge. As we aimed to report only on techniques and procedures comparable to the human situation, we left out all studies reporting on more extreme techniques such as nuclear transfers, in vitro maturation and co-culture with somatic cells. 


\section{RESULTS}

\section{Impact of culture medium on animal birthweight}

Numerous studies have shown that in vitro culture and manipulation procedures affect phenotypic characteristics such as fetal or birthweight in different mammalian species and even result in fetuses weighing several standard deviations more than corresponding control fetuses a phenomenon known as the Large Offspring Syndrome (LOS) in ruminants (see reviews by (Khosla et al., 2001, Young et al., 2001). Many of these studies however, especially those that involve the bovine or ovine model, typically use embryos that are produced in vitro from oocytes recovered from ovaries of slaughtered animals and matured in vitro (IVM). Also embryos produced after pronuclear or somatic cell nuclear transfer or cloning are often used. It is difficult in these studies to distinguish effects that result from in vitro culture from those that result from other procedures. Therefore, in Table I we present only those that used methods similar to the most widely used methods in human IVF thereby excluding studies that used in vitro matured oocytes and nuclear transfer.

Many studies have shown that in vitro culture (IVC) conditions itself in which embryos are cultured during the pre-implantation embryo stages can lead to aberrant fetal growth and development in different mammalian species (see Table I). This effect starts already early in development as it is observed that IVF-embryos reach the blastocyst stage with several hours of delay if compared with in vivo control embryos (Giritharan et al., 2007, Thouas et al., 2003) and ART-embryos of rodents initially implant with significant fewer inner cell mass (ICM) and remain smaller throughout the pregnancy (Bloise et al., 2012, Delle Piane et al., 2010). As early as 1970, differences in birthweight after culture were already reported (Bowman and McLaren, 1970). Following this first publication, several reports appeared on the effects of manipulating the environment of developing embryos. Some studied IVC as a whole, while others compared different culture media with or without supplements such as amino acids, growth factors or serum. Significant changes in birthweight are observed in almost all animal studies, but the direction of impact is depending on the species involved and the type of culture medium or added supplements (Table I). For instance the supplementation of serum to the culture medium causes an increase in fetal weight in sheep (Rooke et al., 2007, Sinclair et al., 1998, Thompson et al., 1995) while in mice the presence of serum leads to a decreased fetal weight (Han and Kiessling, 1988, Khosla et al., 2001). Altogether, results of these animal studies provide growing evidence that the periconceptional period is a window of enhanced sensitivity to environmental conditions like IVC in general, culture media or specific supplements. Although one must be cautious with extrapolating data from animal studies to the human situation, animal studies can give valuable and important information. These findings can support results of human studies, since bias induced by the heterogeneity of a (human) population is prevented. 
Search results of human studies

Fourty-two abstracts were identified by our search strategy. After reading the titles and abstracts, eightteen abstracts were excluded because they were reporting on animals instead of humans and above all not reporting on conventional IVF procedures. Three were excluded for reporting on culture conditions in general, three on postnatal development after ART in general and six were on other topics not related to our search strategy. Eleven articles were relevant and included for our review and will be discussed in this review.

Impact of embryo culture medium on human birthweight

In 2010 our group published the results of the first study comparing birthweight of human offspring born after IVF and as an embryo cultured in two different culture media. In our prospective study, subjects were allocated to culture in either Vitrolife G1.3 PLUS or Cook K-SICM medium by alternating culture media (Dumoulin et al., 2010) (Table II). The allocation procedure was blind to the clinicians who planned ovum pick-ups. All media from both suppliers were ready to use and included $5 \mathrm{mg} / \mathrm{ml}$ pharmaceutical grade human serum albumin (HSA). Assignment was performed by strictly alternating consecutive IVF treatments between the two media types on the day before the ovum pick up by laboratory technicians who were unaware of characteristics of the patients or treatment cycles. In this article, only results of singleton children born after a first IVF treatment cycle were analyzed. Mean birthweight of all live born singletons was significantly lower in the Cook group (Table II). Also the Z-scores, with which birthweight is corrected for gestational age and gender, were significantly different with mean Z-scores being $0.13 \pm 0.09$ for Vitrolife versus $-0.31 \pm 0.10$ for $\operatorname{Cook}(P=0.001)$. After correction for parental weight and height in a regression analysis, culture medium remained a significant predictor of birthweight. In 2012, we expanded the analyses and published results on the outcome of all children (singletons and twins) born after an IVF treatment with fresh or frozen-thawed embryo transfer in any cycle, in the abovementioned period. Similar results were observed with singleton children born after fresh embryo transfer when all cycle ranks are taken into account, with children in the Cook group having a significantly lower mean birthweight than in the Vitrolife group. Also higher intra-twin mean birthweight disparity and birthweight discordance was found in the Cook group if compared to the Vitrolife group. Also a very small group of children born after FET was analyzed, but the observed differences were not statistically significant (Table II) (Nelissen et al., 2012). By examining the data from the ultrasound examinations during pregnancy it appeared that the differences in fetal growth were already present as early as the second trimester of pregnancy (Nelissen et al., 2013). The growth difference persisted at least until the age of 2 years (Kleijkers et al., 2014). 
Recently (2013), the same Vitrolife and Cook culture media (Vitrolife G1.3 and Cook K-SICM) were subject of study in a small randomized study (Carrasco et al., 2013). Sample size calculations were established for comparing clinical pregnancy rates. The birthweight comparison was a post-hoc analysis. A total of 98 live births (49 in each group) were included. Z-scores for birthweight were 0.08 (Vitrolife) and -0.19 (Cook) (Table II). This difference was not significant, which was to be expected with the mentioned sample size. The same effect was also observed by Wunder et al. (2014) in a very recent publication. This retrospective study comparing Cook K-SICM or Vitrolife IVF medium has several limitation including the retrospective design and the fact that the two study groups were of unequal sizes (Cook K-SICM, 173; Vitrolife IVF, 352). The most important limitation of this study is the fact that the two different media were used in two different laboratory sites and the criteria used for patients to be addressed to one of the two sites are unclear. It is also unclear which particular Vitrolife medium was used, as it is only described as Vitrolife IVF. Birthweight of children born after culture in Vitrolife IVF was 127grams [95\%Cl -0.15-253] heavier than children born after culture in Cook K-SICM. Although the differences are not all statistically significant, it is remarkable that the same trends are observed in the studies by Carrasco et al. (2013) and Wunder et al. (2014) with Vitrolife children being heavier than Cook children (Carrasco et al., 2013, Wunder et al., 2014). Carrasco et al. (2013) also performed a retrospective analysis including all cycles within the period of October 2006 - December 2010 where Medicult ISM1, Cook K-SICM and Vitrolife G1.3 were used in consecutive time periods. A total of 523 singleton live births were included in the study. No significant differences between the different culture media were observed for pregnancy rates, live birth rate, miscarriage rates or birthweight.

The same comparison of Cook (Cook K-SICM, $n=974$ ) and Medicult (Medicult embryo Assist, $n=351$ ) was also evaluated in a recent retrospective study published by Lemmen et al. (2014). The culture media were used over a consecutive period of time and during the study period the time of embryo transfer was changed from transfer at day 2 to transfer at day 3. Also the data on the day 2 groups were derived from a single center study and the day 3 groups from a multicenter study. The subgroup of Medicult with transfer at day 3 was also subdivided in a group with and without added granulocyte-macrophage colony-stimulating factor (GM-CSF) making the sizes of the subgroups very small. For the last groups (with and without GM-CSF) the data of a randomized multicenter study by Ziebe et al. (2013) were used (see below). The results of these 4 different groups were compared with outcome of 106842 children from the Danish birth registry. No significant effect on birthweight was observed between the two different culture media, which is in line with the results found by Carrasco et al. (2013). However there was a significant effect of culture medium on birth length with the shortest children in the Medicult D2-group, significantly different from all other groups. Birthweight of all IVF/ICSI children compared to 
the control group was lower as it was to be expected $(P<0.001)$. The subgroup of children born after culture in Medicult with and without GM-CSF was separately analyzed by Ziebe et al. (2013) In a multicenter placebo-controlled, double-blind, prospective randomized study the effect of addition of GM-CSF to culture medium was evaluated. A total of 1332 women were randomized and 304 children (GM-CSF $n=163$, control $n=141$ ) were included for analysis. Ongoing pregnancy rates and live birth rates were significantly higher in the GM-CSF group if compared to the control group (28,9\% (GM-CSF) versus 24.1\% [OR 1.35 $95 \% \mathrm{Cl} 1.03-1.78]$ ). Birthweight and neonatal abnormalities were similar in both groups. These findings are in line with findings from animal studies, suggesting a protective effect of GM-CSF on culture induced embryo stress (Ziebe et al., 2013).

Another recent publication reported results of a comparison between culture media of the same manufacturers as compared by Carrasco et al. in a retrospective analysis. With a large study cohort, significant changes in birthweight were observed after changing the culture medium from Medicult ISM1 to Vitrolife G1.5 PLUS (Eskild et al., 2013). In this large retrospective population based cohort study birthweight of 2435 live born IVF singletons was compared with birthweight of all singletons born after spontaneous conception in Norway during the same years ( $n=698.359$ ). Three different culture media (Medicult Universal, Medicult ISM1, and Vitrolife G1.5 PLUS) were compared after being used consecutively during the study period (1999-2011). The trends for IVF offspring showed in line with the spontaneous population a significant increase in mean placental weight, but in contrast with the spontaneous population, no decrease in mean birthweight was observed (Table II). After the first change of culture medium (Medicult Universal to Medicult ISM1), the crude birthweight in the IVF population decreased more than the birthweight after spontaneous conception during the same period ( $P=0.06$ ) (Table II). After the second change of culture medium (Medicult ISM1 to Vitrolife G1.5 PLUS) the birthweight in IVF pregnancies increased 93 grams more than the birthweight of children born after spontaneous conception $(P<0.05)$. Even after correction for possible confounders the attenuated difference was 79.9 grams between the two IVF groups $(P<0.05)$ (Table II) These results are in contrast with the results reported by Carrasco et al. (2013) (no difference) and Hassani et al. (Hassani et al., 2013) (lower birthweight in Vitrolife group, see below). If overall results are compared, placental weight was significantly higher and birthweight significantly lower after IVF if compared to spontaneous conception (Eskild et al., 2013).

The same media (Medicult ISM1 and Vitrolife G1.5) were also compared by Hassani et al. (2013) in a small prospective randomized study with numerous methodological limitations. They found, in contrast with the findings of Eskild et al., that IVF offspring cultured in Medicult ISM1 was heavier than babies born after culture in Vitrolife G1.5, whereas Eskild et al. reported Vitrolife offspring to be heavier. Randomization protocols and the study 
protocol were not clearly described, also there are inconsistencies in the presented data and results were not corrected for possible confounding factors. Also a different fertilization medium was used as well as another type of oil, which might explain the inconsistent results compared to Eskild et al. (2013). Similar embryo cleavage rates were observed, but the rate of good quality embryos was significantly higher in Medicult ISM1 (42.7\% versus 39\%; $P<0,05$ ). Babies born after culture in Medicult were heavier (3.03 versus $2.66 \mathrm{~kg}$ ) and taller $(48.8 \mathrm{~cm}$ versus $46.0 \mathrm{~cm}$ ) with a P-value of $<0.001$ for both (Hassani et al., 2013).

Eaton et al. (2012) performed a retrospective analysis to evaluate the perinatal outcome after culture in Vitrolife G1.3 and G1.5 and Global (Eaton et al., 2012). The media were used consecutively. Vitrolife G1.3 was utilized from 2003 through 2005 (102 singletons and 172 twins), Global medium from 2005 through 2007 (53 singletons, 58 twins), and Vitrolife G1.5 medium during 2007 and 2008 (43 singletons, 73 twins) with some small overlap. Significant differences between the three groups were found for parity, where women in the Vitrolife G1.3 group were significantly more likely to be nulliparous than women in the other groups. No significant differences in birthweight were observed between the different patient groups, also after correction for possible confounding factors (Table II). The same comparison was made in a relatively large retrospective analysis, comparing the effect of Vitrolife G1.5 ( $n=596$ ) and Global ( $n=460)$, on birthweight and length of newborns, also comparing it with Sage Quinn's advantage ( $n=145)$ (Lin et al., 2013). All culture media were supplemented with corresponding albumin solutions: HSA solution (Vitrolife), HSA solution (IVF online, Global) and Quinn's advantage Serum Protein Subsitute (SPS, Sage). Strength of this study is the large sample size, but the interpretation of the results is complicated by lack of information about the method of allocation. The results of 1201 singletons and 445 sets of twins were analyzed, and no significant differences in birthweight were found (Table II). This is in line with the results of the G1.5-Global comparison of Eaton et al. (2012). Multiple regression analysis showed that maternal weight, maternal length, gestational age and gender of the baby were significantly associated with birthweight. No association between culture medium and birthweight was observed. Paternal length, gestational age and the occurrence of complications were significantly associated with length.

Vergouw et al. (2012) published results of a retrospective cohort study where Human Tubal Fluid (HTF) with 4 mg/ml HSA and Sage Quinn's advantage protein plus medium were compared (Vergouw et al., 2012). In this study again the two media were used in different consecutive time periods (one year HTF followed by three years Sage). Only data from single embryo transfers were included, resulting in 99 and 259 singletons born after culture in HTF and Sage with fresh embryo transfer, respectively, and 32 and 159 singletons respectively born after frozen embryo transfers. In both comparisons no significant 
differences in birthweight were observed, neither after adjustment for gestational age and gender or gestational age and parity (z-scores)(Table II). Significant differences in birthweight were observed if children born after fresh embryo transfer were compared to children born after FET. Mean birthweight was significantly higher in the cryopreservation group, also after adjustment for gestational age, gender and parity.

The effect of addition of certain components to the culture medium was explored by Zhu et al. (2014) and Ziebe et al. (2013) (already discussed). Zhu et al. performed a retrospective study where the effect of addition of human serum albumin (HSA) on birthweight was evaluated. Outcome of 1097 singletons born after fresh embryo transfer was compared after culture in either Vitrolife G1.5 or Vitrolife G1.5PLUS (with HSA added). Although absolute birthweight was not different between the two groups, z-scores were significantly higher for children born after being cultured in Vitrolife G1.5 PLUS if compared to Vitrolife G1.5 $(0.28+1.12$ versus $0.09+1.15$, respectively; $P=0.04)$. Also more LGA babies were born in the Vitrolife G1.5PLUS group (16.8 versus 12.1\%, respectively; $P=0.03$ ) indicating a significant effect of HSA on birthweight of children born after IVF (Zhu et al., 2014) 


\section{DISCUSSION}

Answering the question whether or not there is a true relationship between culture conditions and perinatal outcome is extremely important. It would mean that the environment of the embryo during the first few days after fertilization induces changes in development that have long term impact which is in line with the DOHaD theory.

Several animal studies evaluating the influence of culture (medium) on birthweight have shown that culture (medium) can alter growth and development in animal IVF offspring. However, evidence for an association in human IVF offspring is at this point in time not conclusive. Five out of eleven publications (from 10 different groups) about effect of culture medium on birthweight in human IVF offspring reported an effect (Dumoulin et al., 2010, Eskild et al., 2013, Hassani et al., 2013, Nelissen et al., 2012, Zhu et al., 2014), while others did not. Conventional comparison (meta-analysis) of studies was not possible due to the comparison of different media with other compositions, which makes it hard to compare all different results. In the eleven studies 22 media comparisons were done, with 19 different media couples. Regardless of several methodological issues with some of the reported studies, the diverse composition of the culture media complicates the comparison of studies that compared different media. Most culture media share the same basic components such as a buffer system, mineral salts, carbohydrates and amino acids and most of them have albumin, and antioxidants supplemented (see supplementary data). However, the addition of extra components, alterations in amino acids or carbohydrate compositions and the lack of information on the exact amount of each component in the culture medium complicates the comparison of studies.

For example Vitrolife and Cook are very much alike and comparable with regard to composition. However, an important difference between these media is the fact that in G1.3 medium a stabilized glutamine dipeptide was added, while in Cook instable glutamine was added. Instable glutamine is known to spontaneously break down into ammonium, which is detrimental to human embryos (Gardner et al., 2013, Lane and Gardner, 2003) This could possibly partly explain the lower birthweight observed in children born after culture in Cook if compared to Vitrolife (Carrasco et al., 2013, Dumoulin et al., 2010, Nelissen et al., 2012, Wunder et al., 2014). A comparable difference is also shown by Eskild et al. (2013) where children born after culture in Medicult (instable glutamine) have a lower birthweight than children cultured in Vitrolife (stabilized glutamine). Hassani et al. (2013) found the opposite (Medicult higher BW), but this study has several shortcomings like differences in fertilization medium and mineral oil between the two groups and no correction for any confounding factor. Other media comparisons did not show a difference in the type of glutamine added and no differences in birthweight were observed. 
Another striking difference between Medicult and G1.5 in Eskild study is the amino acids composition. Amino acids play an important role in the homeostatic regulation of embryo development and a wide variety is observed within the different culture media with regard to the amount and the type of amino acids added (Chronopoulou and Harper, 2015). For example Medicult adds a variety of essential amino acids (Histidine, Isoleucine, Lysine, Methionine, Phenylalanine, Tyrosine, Valine), whereas Vitrolife G1.5 only adds Methionine to some of their media. However the culture media compared by Eaton et al. (2012) and Lin et al. (2013) also differed regarding their non-essential amino acids, and no difference in birth weight was found in these studies. Another difference in composition of media that led to birth weight differences was the addition of nucleotides, cholesterol and malic acid in Origio Medicult. It is not stated by the manufacturers what the function of these components could be and literature on this topic is scarce.

The albumin source and the way of supplementation (ready to use media with HSA already added versus addition of HSA prior to usage) could also play an important role in the observed differences as was clearly shown by Zhu et al. (2014).

There are some additional difficulties with the analysis in human studies if compared to animal studies. The often consecutive use of the different culture media is one of the most important limitations. As mentioned, except for the Dumoulin study (2010), the small prospective study of Carrasco et al. (2013) and Hassani et al. (2013) all studies compared the different culture media after consecutive use of the different media. This makes it difficult to exclude the influence of other factors that might have changed over time such as changed culture conditions (e.g. oxygen concentration and temperature), technical procedures, stimulation protocols and altered population characteristics. The influence of time on population characteristics and altered growth patterns in the general population was clearly shown by Eskild et al. (2013). By comparing the trends for birthweight and placental weight in the IVF population with these trends in the spontaneous population, they were able to correct for time-associated changes in population characteristics and found significant associations between birthweight and culture medium used. Most studies used a retrospective design where different culture media were used over consecutive periods of time, which makes bias likely because it is not possible to correct for all changes in treatment protocols and population characteristics.

Next to the retrospective design and use of culture media over different time periods, most studies had relatively small sample sizes, which makes it hard, to detect smaller differences. To detect a difference in birthweight of 100 grams, with a standard deviation of 500grams, $a \leq 0.05$ and a power of $80 \%, 393$ children in each group are required. Thus with a hypothesized live birth rate of 25\%, 1600 cycles resulting in 400 children in each 
group are required to show a significant difference in birthweight (www.openepi.com). Of the reported studies, only Lin et al. (2013) and Eskild et al. (2013) met these sample sizes. All other studies had sample sizes that allowed only larger differences in birthweight to be detected (e.g. for 200 grams 99 children in each group are needed and for 250 grams 63 children).

Apart from the sample sizes being too small, not all results were easy to compare. Not all studies used z-scores to correct for gestational age and gender, making interpretation of the observed differences difficult. Also correction for other confounding factors was limited (probably due to the post-hoc study design) and not described structurally in all studies. For example, the studies of Carrasco et al. (2013), Vergouw et al. (2012) and Eaton et al. (2012) only mentioned which possible confounders were included in the regression analysis, but only gave results for culture medium and not the actual results of the regression analysis which would make clear which other factors affect birthweight (Table II). Also the number of confounders corrected for and the type of confounders varied among the different studies. Smoking, parity, socio-economic status, sex distribution and gestational age could be important confounding factors for birthweight, but these data were missing in most studies (Table II). Maternal age, parity, childs' gender and gestational age were significant confounders in several studies, but not all studies included these in their regression analysis.

When culture or culture medium indeed affects perinatal outcome, this puts into question the safety of culture length and blastocyst culture. Zhu et al. showed that absolute mean birthweight of singletons born after blastocyst transfer was significantly higher than that of singletons born from day 3 embryo transfer (Zhu et al., 2014). These findings are in line with earlier studies showing differences in perinatal outcome if length of culture is compared. For example also the study of Makinen et al. (2013) showed a significant increase in the proportion of large for gestational age (LGA) babies after blastocyst transfer if compared with day 2 transfer (Makinen et al., 2013).

If a relation between culture medium and fetal growth and development would be confirmed, the elucidation of the mechanisms behind this association is of high interest. Well known is the Barker Hypothesis (Barker, 2006), describing the inverse correlation between birthweight and the development of chronic diseases in later life. The in vitro culture can, next to an effect on fetal growth and birthweight (Table I), also have an effect on a variety of other important parameters like glucose metabolism and leptin levels (Feuer et al., 2014, Scott et al., 2010), changes in blood pressure and vascular problems (Rexhaj et al., 2013, Watkins et al., 2007), changes in gene expression (Calle et al., 2012, Giritharan et al., 2007) and abnormal imprinting marks (Doherty et al., 2000). It can also cause altered pregnancy 
chances, have effects on perinatal outcome and mice born after ART have been shown to exhibit abnormal behavior (Ecker et al., 2004, Fernández-Gonzalez et al., 2004, Feuer et al., 2014, Rexhaj et al., 2013). Regarding the young age of human IVF progeny yet, it is not clear whether such a relation also holds true for the IVF population. In human studies evidence on long-term effects of IVC is more limited but postnatal effects in line with animals observations have already been observed. Children born after ART are more likely to have behavioral problems (Zhan et al., 2013), alterations in cardio metabolic parameters like a higher blood pressure, altered lipid profile or impaired glucose tolerance (Ceelen et al., 2008, Scherrer et al., 2012) and moreover an altered expression of imprinted genes and occurrence of imprinting disorders is observed (Lim et al., 2009, Vermeiden and Bernardus, 2013). In this respect it is also interesting that different human culture media can induce different gene expressions and DNA methylation patterns in various animal models (Market-Velker et al., 2010, Schwarzer et al., 2012).

Results of the Dutch Famine studies underline that even without any effect on birthweight, exposure to suboptimal conditions such as poor nutrition during early development leads to large and long-term negative consequences for both mental and physical health (Roseboom et al., 2011). The observations of a lower birthweight in IVF children (either or not induced by culture medium) therefore raises concerns on the health of the IVF children and requires careful monitoring of this population. 


\section{CONCLUSION}

From animal studies, there is extensive evidence confirming the hypothesis that not only infertility, stimulation protocols and patient characteristics can influence pregnancy outcome and birthweight, but also that the embryo culture medium with its own particular composition has an important effect on perinatal outcome and birthweight. Although extreme differences in birthweight as observed in animal studies have not been observed in humans, the current evidence indicates a possible similar relationship between certain culture medium types and birthweight in human. Up until today, there are eleven human studies published on this topic of which five studies reported a significant difference. Although these five studies have among the highest sample sizes and the best study designs, all reported studies have methodological limitations. Results of all studies are hard to compare because different culture media with its own composition are compared, making it an uneven comparison. As a result, the actual effect of culture medium on growth of IVF offspring is still not definitive elucidated and more research might have to focus on different ingredients of the culture media. Further research on this relationship is indispensable because of the possible far-reaching post-natal health consequences for IVF offspring. Therefore, parallel with more prospective randomized controlled trials to confirm the effect of culture medium on growth, more research is required investigating effects and long-term safety of the IVF technique and culture medium on health and epigenetic (de)regulation of IVF offspring. 
Table I - Selection of animal studies reporting on effect of culture (medium) on birthweight

\begin{tabular}{|c|c|c|c|}
\hline Study & Species & Study group & Culture media used \\
\hline \multicolumn{4}{|l|}{ Mouse Studies } \\
\hline Le et al. 2013 & Mouse & OS+IVF & HTF \\
\hline Hemkemeyer et al. 2013 & Mouse & OS+IVD 1-C & KSOM(AA), WM, ISM1/2, HTF/Multiblast \\
\hline Delle Piane et al. 2010 & Mouse & OS+IVF & WM or $\mathrm{KSOM}(\mathrm{AA})$ \\
\hline Scott et al. 2010 & Mouse & OS+IVF & CZB \\
\hline Zander et al. 2006 & Mouse & OS+IVD 1-C & G1.2/G2.2 w/wo added ammonium \\
\hline Sjöblom et al. 2005 & Mouse & $\mathrm{NO}+$ IVD $2-\mathrm{C}$ & SQC w/WO GM-CSF \\
\hline Thouas et al. 2003 & Mouse & OS+IVD 1-C & $\mathrm{MTF}+\mathrm{BSA}$ \\
\hline Khosla et al. 2001 & Mouse & OS+IVD 1-C & M16 w/wo serum \\
\hline Lane \& Gardner 1997 & Mouse & OS+IVD 1-C & mMTF w/wo AA \\
\hline Gardner \& Lane 1996 & Mouse & OS+IVD 1-C & CZB or DM1/DM2 \\
\hline Mehta \& Kiessling, 1990 & Mouse & OS+IVF & EBSS w/wo EDTA, AA, serum \\
\hline Han \& Kiessling, 1988 & Mouse & OS+IVF & EBSS or Ham's F10 w/wo serum \\
\hline Bowman \& McLaren, 1970 & Mouse & NS+IVD 8-C & Brinster medium wo AA \\
\hline \multicolumn{4}{|l|}{ Ovine studies } \\
\hline Sinclair et al. 1999 & Ovine & OS+IVD 1-C & SOF w/wo serum \\
\hline Rooke et al. 2007 & Ovine & OS+IVD 1-C & SOF w/wo serum \\
\hline Walker et al. 1992 & Ovine & OS+IVD 1-C & - \\
\hline Thompson et al. 1995 & Ovine & OS+IVD 1-C & SOF w/wo AA and serum or BSA \\
\hline
\end{tabular}

$B W=$ birthweight, $F W=$ fetal weight, $I V C=$ in vitro culture, $I V D=$ in vivo developed, $O S=$ ovarian stimulation 


\begin{tabular}{|c|c|c|}
\hline Control group & Transferred at & Effect of IVC vs control \\
\hline OS+IVD 2-cell & 2-cell & Increased BW \\
\hline OS+IVD to fetus & B stage & Increased BW \\
\hline OS+IVD B'S & B stage & Reduced FW, less pronounced in KSOM-AA \\
\hline IVD to fetus & 2-cell & Increased BW \\
\hline OS+IVD B'S & B stage & Reduced FW, only in the presence of ammonium \\
\hline $\mathrm{NO}+$ IVD B'S & B stage & Reduced FW, less pronounced in presence of GM-CSF \\
\hline OS+IVD B'S & B stage & Increased FW \\
\hline OS+IVD B'S & B stage & Reduced FW in presence of serum \\
\hline OS+IVD D6 embryos & B stage & Reduced FW \\
\hline OS+IVD D6 embryos & B stage & Reduced FW dependent on medium type \\
\hline OS+IVD 2-cell & 4-cell (IVC), 2-cell (control) & Reduced FW dependent on EDTA concentration and AA \\
\hline OS+IVD 2-cell & 4-cell (IVC), 2-cell (control) & Reduced FW in presence of serum \\
\hline NS+IVD B'S & B stage & Reduced FW dependent on time in culture \\
\hline OS+IVD D6 embryos & B stage & Increased FW \\
\hline OS+IVD B'S & B stage & Increased FW in presence of serum during the first $48 \mathrm{~h}$ of IVC \\
\hline OS+IVD B'S & B stage & Increased BW \\
\hline OS+IVD to birth & B stage & Increased BW in presence of serum \\
\hline
\end{tabular}


Tabel II - Characteristics and results of studies investigating the effect of human embryo culture media on birthweight*

\begin{tabular}{llllll}
\hline Study & Design & Medium & CL & Period & NLB \\
\hline $\begin{array}{l}\text { Dumoulin et } \\
\text { al. } 2010\end{array}$ & Prospective with random allocation & Vitrolife G1.3 & 2-3 & july '03-dec '06 & 110 \\
& & & &
\end{tabular}

Cook K-SICM 2-3 july '03-dec '06 78

Nelissen et al. $\quad$ Prospective with random allocation Vitrolife G1.3 $\quad 2-3 \quad$ july '03-dec '06 168 2012

Cook K-SICM 2-3 july '03-dec '06 126

\begin{tabular}{|c|c|c|c|c|c|}
\hline \multirow{5}{*}{$\begin{array}{l}\text { Carrasco et al. } \\
2013\end{array}$} & Prospective with randomisation & Vitrolife G1.3 & 2 & aug-dec '08 & 49 \\
\hline & & Cook K-SICM & 2 & & 49 \\
\hline & Retrospective Cohort & Medicult ISM1 & 2 & oct ‘06 - mei ‘07 & 172 \\
\hline & & Cook K-SICM & 2 & July '07 -dec'07 & 154 \\
\hline & & Vitrolife G1.3 & 2 & Feb '09 - dec'10 & 197 \\
\hline
\end{tabular}




\begin{tabular}{|c|c|c|c|c|c|}
\hline BW (g) (SD) & P-value BW & Z-score (SD) & $\begin{array}{l}\text { P-value } \\
\text { z-score }\end{array}$ & Regression Analysis & $\begin{array}{l}\text { Result regression } \\
\text { analysis }\end{array}$ \\
\hline \multirow[t]{5}{*}{$3453(556)$} & 0.003 & $0.13(0,09)$ & 0.001 & Culture medium & $S(P<0.01)$ \\
\hline & & & & Maternal age & $\mathrm{S}(P<0.0001)$ \\
\hline & & & & Parity & $\mathrm{S}(P<0.005)$ \\
\hline & & & & Childs' gender & $\mathrm{S}(P<0.03)$ \\
\hline & & & & Gestational age & $\mathrm{S}(P<0.03)$ \\
\hline \multirow[t]{6}{*}{3208 (539) } & & $-0.31(0.10)$ & & Parental weight & NS \\
\hline & & & & Parental height & NS \\
\hline & & & & Smoking habits & NS \\
\hline & & & & Duration of subfertility & NS \\
\hline & & & & Time of ET & NS \\
\hline & & & & No of embryos transferred & NS \\
\hline \multirow[t]{7}{*}{$3436(570)$} & 0.006 & $0.05(0.08)$ & 0.007 & Culture medium & $S(P=0.03)$ \\
\hline & & & & Gestational age & $S(P<0.0001)$ \\
\hline & & & & Childs' gender & $S(P<0.001)$ \\
\hline & & & & Secondary subfertility & $S(P<0.001)$ \\
\hline & & & & Maternal height & $\mathrm{S}(P<0.008)$ \\
\hline & & & & Fertilization method & NS \\
\hline & & & & Maternal weight & NS \\
\hline \multirow[t]{7}{*}{$3253(561)$} & & $0.265(0.08)$ & & Height and weight of father & NS \\
\hline & & & & Smoking & NS \\
\hline & & & & Age & NS \\
\hline & & & & $\begin{array}{l}\text { Number of transferred } \\
\text { embryos, day of ET }\end{array}$ & NS \\
\hline & & & & Duration of subfertility & NS \\
\hline & & & & Cycle number & NS \\
\hline & & & & Pregnancy complications & NS \\
\hline \multirow[t]{2}{*}{3215.5} & 0.98 & $0.08(1.40)$ & 0.23 & Culture medium & NS $(P>0.05)$ \\
\hline & & & & Maternal age & NS $(P>0.05)$ \\
\hline 3154.7 & & $-0.19(0.85)$ & & BMI & NS $(P>0.05)$ \\
\hline 3155.8 & 0.68 & $0.06(1.13)$ & 0.68 & Culture medium & NS $(P>0.05)$ \\
\hline 3127.9 & & $-0.14(0.96)$ & & Maternal age & NS $(P>0.05)$ \\
\hline 3105.6 & & $0.03(1.05)$ & & BMI & NS $(P>0.05)$ \\
\hline
\end{tabular}


Tabel II - Continued

\begin{tabular}{|c|c|c|c|c|c|}
\hline Study & Design & Medium & $\mathrm{CL}$ & Period & NLB \\
\hline \multirow{3}{*}{$\begin{array}{l}\text { Lemmen et al. } \\
2014\end{array}$} & \multirow{3}{*}{$\begin{array}{l}\text { Retrospective Cohort compared } \\
\text { with naturally conceived singletons }\end{array}$} & Cook K-SICM & 2 & 04-'09 & 977 \\
\hline & & $\begin{array}{l}\text { Medicult } \\
\text { Embryo Assist }\end{array}$ & 2 & 10 & 147 \\
\hline & & $\begin{array}{l}\text { Medicult } \\
\text { Embryo Assist } \\
\text { with and } \\
\text { without GM- } \\
\text { CSF }\end{array}$ & 3 & 07-'10 & 204 \\
\hline \multirow[t]{2}{*}{ Ziebe et al. } & \multirow[t]{2}{*}{$\begin{array}{l}\text { Multicenter randomized } \\
\text { placebo-controlled double-blind } \\
\text { prospective }\end{array}$} & $\begin{array}{l}\text { Medicult } \\
\text { Embryo Assist } \\
\text { with GM-CSF }\end{array}$ & 3 & nov'07 - march '11 & 163 \\
\hline & & $\begin{array}{l}\text { Medicult } \\
\text { Embryo Assist } \\
\text { without GM- } \\
\text { CSF (control) }\end{array}$ & 3 & & 141 \\
\hline \multirow[t]{3}{*}{$\begin{array}{l}\text { Eskild et al. } \\
2013\end{array}$} & \multirow[t]{3}{*}{$\begin{array}{l}\text { Retrospective cohort with matched } \\
\text { controls }\end{array}$} & $\begin{array}{l}\text { Medicult } \\
\text { Universal }\end{array}$ & $1-2$ & '99- dec '07 & 1584 \\
\hline & & Medicult ISM1 & $1-2$ & Jan '08 - sept '09 & 402 \\
\hline & & Vitrolife G1.5 & $1-2$ & sept ‘09- '11 & 449 \\
\hline \multirow[t]{3}{*}{$\begin{array}{l}\text { Eaton et al. } \\
2012\end{array}$} & \multirow[t]{3}{*}{ Retrospective cohort } & Vitrolife G1.3 & - & ‘03-05 & 102 \\
\hline & & Global & - & '05-'07 & 53 \\
\hline & & Vitrolife G1.5 & - & '07-'08 & 43 \\
\hline \multirow{2}{*}{$\begin{array}{l}\text { Hassani et al. } \\
2013\end{array}$} & \multirow[t]{2}{*}{ Randomized prospective } & Medicult ISM1 & $2-3$ & Feb-june '09 & 86 \\
\hline & & Vitrolife G1.5 & $2-3$ & Feb-june '09 & 78 \\
\hline
\end{tabular}




\begin{tabular}{|c|c|c|c|c|c|}
\hline BW (g) (SD) & P-value BW & Z-score (SD) & $\begin{array}{l}\text { P-value } \\
z \text {-score }\end{array}$ & Regression Analysis & $\begin{array}{l}\text { Result regression } \\
\text { analysis }\end{array}$ \\
\hline $3367.6(610)$ & 0.302 & $20.2(1.14)$ & 0.402 & Culture medium & NS $(P=0.426)$ \\
\hline $3312.5(552)$ & & $20.3(0.99)$ & & Gestational length & $S(P<0.001)$ \\
\hline \multirow[t]{3}{*}{3351.9 (637) } & 0.547 & $20.3(1.14)$ & 0.605 & Childs' gender & $S(P<0.001)$ \\
\hline & & & & Maternal age & NS $(P=0.697)$ \\
\hline & & & & $\begin{array}{l}\text { No. Of embryos } \\
\text { transferred }\end{array}$ & NS $(P=0.107)$ \\
\hline 3275 (936) & 0.54 & - & - & - & NS \\
\hline \multicolumn{6}{|l|}{3297 (587) } \\
\hline \multirow[t]{3}{*}{$3448(611)$} & 0.02 & - & - & Culture medium & $S(P=0.020)$ \\
\hline & & & & Maternal age & s \\
\hline & & & & Parity & s \\
\hline \multirow[t]{2}{*}{3352 (631) } & & & & Gestational age & s \\
\hline & & & & Year of birth & NS \\
\hline \multirow[t]{2}{*}{3444 (637) } & & & & $\begin{array}{l}\text { number of transferred } \\
\text { embryos }\end{array}$ & NS \\
\hline & & & & Maternal BMI & NS \\
\hline \multirow[t]{3}{*}{3368 (569) } & 0.69 & - & - & Culture medium & ND \\
\hline & & & & Maternal age & ND \\
\hline & & & & Parity & ND \\
\hline \multirow[t]{3}{*}{3331 (441) } & & & & Childs'gender & ND \\
\hline & & & & Maternal age & ND \\
\hline & & & & Gestational age & ND \\
\hline \multirow[t]{3}{*}{$3273(534)$} & & & & Fertilization method & ND \\
\hline & & & & Use of assisted hatching & ND \\
\hline & & & & Embryo quality & ND \\
\hline $3030(70)$ & 0.001 & - & - & - & ND \\
\hline $2660(80)$ & & - & & & \\
\hline
\end{tabular}


Tabel II - Continued

\begin{tabular}{|c|c|c|c|c|c|}
\hline Study & Design & Medium & $\mathrm{CL}$ & Period & NLB \\
\hline \multirow[t]{3}{*}{ Lin et al. 2013} & Retrospective cohort & $\begin{array}{l}\text { Vitrolife G1.5 } \\
\text { supplemented } \\
\text { with VL HSA } \\
\text { solution }\end{array}$ & 3 & unclear '08-'10 & 596 \\
\hline & & $\begin{array}{l}\text { Global } \\
\text { supplemented } \\
\text { with Global HSA }\end{array}$ & 3 & unclear '08-'10 & 460 \\
\hline & & $\begin{array}{l}\text { Sage Quinn's } \\
\text { advantage, } \\
\text { supplemented } \\
\text { with Quinn's } \\
\text { advantage SPS }\end{array}$ & 3 & unclear '08-'10 & 145 \\
\hline
\end{tabular}

Vergouw et al. Retrospective cohort 2012

HTF

3 '08-'09

99

$\begin{array}{llll}\text { Sage Quinn's } & 3 & \text { '09-11 }\end{array}$

advantage

protein plus

\begin{tabular}{|c|c|c|c|c|}
\hline $\begin{array}{l}\text { Zhu et al. } \\
(2014)\end{array}$ & Retrospective cohort & $\begin{array}{l}\text { Vitrolife G1.5 } \\
\text { supplemented } \\
\text { with VL HSA } \\
\text { solution }\end{array}$ & 3 & jan '11 - aug '12 \\
\hline
\end{tabular}

Vitrolife

G1.5PLUS

\footnotetext{
*Only results of singletons born after fresh embryo transfer are reported in this table.

CL - culture length; NLB - number of live births; BW - birthweight; SD - standard deviation; S - significant; NS - not significant; ND - not described.
} 


\begin{tabular}{|c|c|c|c|c|c|}
\hline BW (g) (SD) & P-value BW & Z-score (SD) & $\begin{array}{l}\text { P-value } \\
z \text {-score }\end{array}$ & Regression Analysis & $\begin{array}{l}\text { Result regression } \\
\text { analysis }\end{array}$ \\
\hline \multirow[t]{4}{*}{$3246(540)$} & 0.327 & - & - & Culture Medium & NS \\
\hline & & & & Maternal height & $S(P=0.013)$ \\
\hline & & & & Maternal weight & $S(P=0.000)$ \\
\hline & & & & Gestational age & $\mathrm{S}(P=0.000)$ \\
\hline \multirow[t]{3}{*}{3294 (564) } & & & & Childs' Gender & $\mathrm{S}(P=0.002)$ \\
\hline & & & & Fertilization method & NS \\
\hline & & & & Paternal weight and height & NS \\
\hline \multirow[t]{4}{*}{3291 (524) } & & & & $\begin{array}{l}\text { Type, duration and cause of } \\
\text { subfertility }\end{array}$ & NS \\
\hline & & & & $\begin{array}{l}\text { Number of transferred } \\
\text { embryos }\end{array}$ & NS \\
\hline & & & & Number of cycles & NS \\
\hline & & & & Newborn complication & NS \\
\hline \multirow[t]{4}{*}{3446 (508) } & 0.11 & $0.04(0.87)$ & 0.67 & Culture medium & NS \\
\hline & & & & Gestational age & s \\
\hline & & & & Parity & s \\
\hline & & & & Childs' gender & s \\
\hline \multirow[t]{3}{*}{$3341(575)$} & & $0.09(0.99)$ & & maternal age & NS \\
\hline & & & & BMI & NS \\
\hline & & & & Smoking & NS \\
\hline \multirow[t]{7}{*}{ 3333.2(491) } & NS & $0.09(1.15)$ & 0.04 & Culture medium & $S(P=0.04)$ \\
\hline & & & & Male height & $S(P=0.04)$ \\
\hline & & & & Female weight & $S(P=0.00)$ \\
\hline & & & & Gestational age (weeks) & $\mathrm{S}(P=0.00)$ \\
\hline & & & & Childs' gender & $S(P=0.00)$ \\
\hline & & & & Female age & NS \\
\hline & & & & Male age & NS \\
\hline \multirow[t]{6}{*}{$3375.9(479)$} & & $0.28(1.12)$ & & Female height & NS \\
\hline & & & & Duration of infertility & NS \\
\hline & & & & Type of infertility & NS \\
\hline & & & & Male weight & NS \\
\hline & & & & Cycle number & NS \\
\hline & & & & $\begin{array}{l}\text { Ovarian stimulation } \\
\text { (agonist versus antagonist) }\end{array}$ & NS \\
\hline
\end{tabular}




\section{REFERENCES}

Barker DJ. Adult consequences of fetal growth restriction. Clinical obstetrics and gynecology 2006; 49:270-283.

Barker DJP. The origins of the developmental origins theory. J Intern Med 2007; 261:412-417.

Bloise E, Feuer SK and Rinaudo PF. Comparative intrauterine development and placental function of ART concepti: implications for human reproductive medicine and animal breeding. Human reproduction update 2014 20:822-839.

Bloise E, Lin W, Liu X, Simbulan R, Kolahi KS, Petraglia F, Maltepe E, Donjacour A and Rinaudo P. Impaired placental nutrient transport in mice generated by in vitro fertilization. Endocrinology 2012; 153:3457-3467.

Bowman P and McLaren A. Viability and growth of mouse embryos after in vitro culture and fusion. Journal of embryology and experimental morphology 1970; 23:693-704.

Calle A, Fernandez-Gonzalez R, Ramos-Ibeas P, Laguna-Barraza R, Perez-Cerezales S, Bermejo-Alvarez P, Ramirez MA and Gutierrez-Adan A. Long-term and transgenerational effects of in vitro culture on mouse embryos. Theriogenology 2012; 77:785-793.

Carrasco B, Boada M, Rodriguez I, Coroleu B, Barri PN and Veiga A. Does culture medium influence offspring birth weight? Fertility and sterility 2013; 100:1283-1288.

Ceelen M, van Weissenbruch MM, Vermeiden JP, van Leeuwen FE and Delemarre-van de Waal HA. Growth and development of children born after in vitro fertilization. Fertility and sterility 2008; 90:1662-1673.

Chronopoulou E and Harper JC. IVF culture media: past, present and future. Human reproduction update 2015; 21:39-55.

Delle Piane L, Lin W, Liu X, Donjacour A, Minasi P, Revelli A, Maltepe E and Rinaudo PF. Effect of the method of conception and embryo transfer procedure on mid-gestation placenta and fetal development in an IVF mouse model. Human reproduction 2010; 25:2039-2046.

Doherty AS, Mann MRW, Tremblay KD, Bartolomei MS and Schultz RM. Differential effects of culture on imprinted H19 expression in the preimplantation mouse embryo. Biol Reprod 2000; 62:1526-1535.

Dumoulin JC, Land JA, Van Montfoort AP, Nelissen EC, Coonen E, Derhaag JG, Schreurs IL, Dunselman GA, Kester $A D$, Geraedts JP et al. Effect of in vitro culture of human embryos on birthweight of newborns. Human reproduction 2010; 25:605-612.

Eaton JL, Lieberman ES, Stearns C, Chinchilla M and Racowsky C. Embryo culture media and neonatal birthweight following IVF. Human reproduction 2012; 27:375-379.

Ecker DJ, Stein P, Xu Z, Williams CJ, Kopf GS, Bilker WB, Abel T and Schultz RM. Long-term effects of culture of preimplantation mouse embryos on behavior. Proceedings of the National Academy of Sciences of the United States of America 2004; 101:1595-1600.

Eskild A, Monkerud L and Tanbo T. Birthweight and placental weight; do changes in culture media used for IVF matter? Comparisons with spontaneous pregnancies in the corresponding time periods. Human reproduction 2013; 28:3207-3214.

Fernandez-Gonzalez R, Moreira P, Bilbao A, Jimenez A, Perez-Crespo M, Ramirez MA, Rodriguez De Fonseca F, Pintado B and Gutierrez-Adan A. Long-term effect of in vitro culture of mouse embryos with serum on mRNA expression of imprinting genes, development, and behavior. Proceedings of the National Academy of Sciences of the United States of America 2004; 101:5880-5885.

Feuer SK, Liu X, Donjacour A, Lin W, Simbulan RK, Giritharan G, Piane LD, Kolahi K, Ameri K, Maltepe E et al. Use of a Mouse in Vitro Fertilization Model to Understand the Developmental Origins of Health and Disease Hypothesis. Endocrinology 2014:en20132081.

Gardner DK, Hamilton R, McCallie B, Schoolcraft WB and Katz-Jaffe MG. Human and mouse embryonic development, metabolism and gene expression are altered by an ammonium gradient in vitro. Reproduction 2013; 146:49-61. 
Gardner DK and Lane M. Alleviation of the '2-cell block' and development to the blastocyst of CF1 mouse embryos: role of amino acids, EDTA and physical parameters. Human reproduction 1996; 11:2703-2712.

Gibbons WE, Cedars M, Ness RB and Society for Assisted Reproductive Technologies Writing G. Toward understanding obstetrical outcome in advanced assisted reproduction: varying sperm, oocyte, and uterine source and diagnosis. Fertility and sterility 2011; 95:1645-1649 e1641.

Gillman MW, Barker D, Bier D, Cagampang F, Challis J, Fall C, Godfrey K, Gluckman P, Hanson M, Kuh D et al. Meeting report on the 3rd International Congress on Developmental Origins of Health and Disease (DOHaD). Pediatric research 2007; 61:625-629.

Giritharan G, Talbi S, Donjacour A, Di Sebastiano F, Dobson AT and Rinaudo PF. Effect of in vitro fertilization on gene expression and development of mouse preimplantation embryos. Reproduction 2007; 134:63-72.

Godfrey KM, Gluckman PD and Hanson MA. Developmental origins of metabolic disease: life course and intergenerational perspectives. Trends in endocrinology and metabolism: TEM 2010; 21:199-205.

Griesinger G, Kolibianakis EM, Diedrich K and Ludwig M. Ovarian stimulation for IVF has no quantitative association with birthweight: a registry study. Human reproduction 2008; 23:2549-2554.

Han HD and Kiessling AA. In vivo development of transferred mouse embryos conceived in vitro in simple and complex media. Fertility and sterility 1988; 50:159-163.

Hassani F, Eftekhari-Yazdi P, Karimian L, Rezazadeh Valojerdi M, Movaghar B, Fazel M, Fouladi HR, Shabani F and Johansson L. The Effects of ISM1 Medium on Embryo Quality and Outcomes of IVF/ICSI Cycles. International journal of fertility \& sterility 2013; 7:108-115.

Helmerhorst FM, Perquin DA, Donker D and Keirse MJ. Perinatal outcome of singletons and twins after assisted conception: a systematic review of controlled studies. Bmj 2004; 328:261.

Hemkemeyer SA, Schwarzer C, Boiani M, Ehmcke J, Le Gac S, Schlatt S and Nordhoff V. Effects of embryo culture media do not persist after implantation: a histological study in mice. Human reproduction 2014; 29:220-233.

Henningsen AK, Pinborg A, Lidegaard O, Vestergaard C, Forman JL and Andersen AN. Perinatal outcome of singleton siblings born after assisted reproductive technology and spontaneous conception: Danish national sibling-cohort study. Fertility and sterility 2011; 95:959-963.

Imudia AN, Awonuga AO, Kaimal AJ, Wright DL, Styer AK and Toth TL. Elective cryopreservation of all embryos with subsequent cryothaw embryo transfer in patients at risk for ovarian hyperstimulation syndrome reduces the risk of adverse obstetric outcomes: a preliminary study. Fertility and sterility 2013; 99:168-173.

Jackson RA, Gibson KA, Wu YW and Croughan MS. Perinatal outcomes in singletons following in vitro fertilization: a meta-analysis. Obstetrics and gynecology 2004; 103:551-563.

Khosla S, Dean W, Reik W and Feil R. Culture of preimplantation embryos and its long-term effects on gene expression and phenotype. Human reproduction update 2001; 7:419-427.

Kleijkers SH, van Montfoort AP, Smits LJ, Viechtbauer W, Roseboom TJ, Nelissen EC, Coonen E, Derhaag JG, Bastings $L$, Schreurs IE et al. IVF culture medium affects post-natal weight in humans during the first 2 years of life. Human reproduction 2014; 29:661-669.

Lane M and Gardner DK. Nonessential amino acids and glutamine decrease the time of the first three cleavage divisions and increase compaction of mouse zygotes in vitro. Journal of assisted reproduction and genetics 1997; 14:398-403.

Lane M and Gardner DK. Ammonium induces aberrant blastocyst differentiation, metabolism, pH regulation, gene expression and subsequently alters fetal development in the mouse. Biol Reprod 2003; 69:1109-1117.

Le F, Wang LY, Wang N, Li L, Li le J, Zheng YM, Lou HY, Liu XZ, Xu XR, Sheng JZ et al. In vitro fertilization alters growth and expression of Igf2/H19 and their epigenetic mechanisms in the liver and skeletal muscle of newborn and elder mice. Biol Reprod 2013; 88:75. 
Lemmen JG, Pinborg A, Rasmussen S and Ziebe S. Birthweight distribution in ART singletons resulting from embryo culture in two different culture media compared with the national population. Human reproduction 2014; 29:2326-2332.

Lim D, Bowdin SC, Tee L, Kirby GA, Blair E, Fryer A, Lam W, Oley C, Cole T, Brueton LA et al. Clinical and molecular genetic features of Beckwith-Wiedemann syndrome associated with assisted reproductive technologies. Human reproduction 2009; 24:741-747.

Lin S, Li M, Lian Y, Chen L and Liu P. No effect of embryo culture media on birthweight and length of newborns. Human reproduction 2013; 28:1762-1767

Makinen S, Soderstrom-Anttila V, Vainio J, Suikkari AM and Tuuri T. Does long in vitro culture promote large for gestational age babies? Human reproduction 2013; 28:828-834.

Malchau SS, Loft A, Larsen EC, Aaris Henningsen AK, Rasmussen S, Andersen AN and Pinborg A. Perinatal outcomes in 375 children born after oocyte donation: a Danish national cohort study. Fertility and sterility 2013; 99:1637-1643.

Marino JL, Moore VM, Willson KJ, Rumbold A, Whitrow MJ, Giles LC and Davies MJ. Perinatal outcomes by mode of assisted conception and sub-fertility in an Australian data linkage cohort. PloS one 2014; 9:e80398.

Market-Velker BA, Fernandes AD and Mann MR. Side-by-side comparison of five commercial media systems in a mouse model: suboptimal in vitro culture interferes with imprint maintenance. Biol Reprod 2010; 83:938-950.

McDonald SD, Han Z, Mulla S, Murphy KE, Beyene J, Ohlsson A and Knowledge Synthesis G. Preterm birth and low birth weight among in vitro fertilization singletons: a systematic review and meta-analyses. European journal of obstetrics, gynecology, and reproductive biology 2009; 146:138-148.

Mehta TS and Kiessling AA. Development potential of mouse embryos conceived in vitro and cultured in ethylenediaminetetraacetic acid with or without amino acids or serum. Biol Reprod 1990; 43:600-606.

Messerlian C, Maclagan L and Basso $O$. Infertility and the risk of adverse pregnancy outcomes: a systematic review and meta-analysis. Human reproduction 2013; 28:125-137.

Nakashima A, Araki R, Tani H, Ishihara O, Kuwahara A, Irahara M, Yoshimura Y, Kuramoto T, Saito H, Nakaza A et al. Implications of assisted reproductive technologies on term singleton birth weight: an analysis of 25,777 children in the national assisted reproduction registry of Japan. Fertility and sterility 2013; 99:450-455.

Nelissen EC, Van Montfoort AP, Coonen E, Derhaag JG, Geraedts JP, Smits LJ, Land JA, Evers JL and Dumoulin JC. Further evidence that culture media affect perinatal outcome: findings after transfer of fresh and cryopreserved embryos. Human reproduction 2012; 27:1966-1976.

Nelissen EC, Van Montfoort AP, Smits LJ, Menheere PP, Evers JL, Coonen E, Derhaag JG, Peeters LL, Coumans AB and Dumoulin JC. IVF culture medium affects human intrauterine growth as early as the second trimester of pregnancy. Human reproduction 2013; 28:2067-2074.

Pandey S, Shetty A, Hamilton M, Bhattacharya S and Maheshwari A. Obstetric and perinatal outcomes in singleton pregnancies resulting from IVF/ICSI: a systematic review and meta-analysis. Human reproduction update 2012; 18:485-503.

Pelinck MJ, Hadders-Algra M, Haadsma ML, Nijhuis WL, Kiewiet SM, Hoek A, Heineman MJ and Middleburg KJ. Is the birthweight of singletons born after IVF reduced by ovarian stimulation or by IVF laboratory procedures? Reproductive biomedicine online 2010; 21:245-251.

Pinborg A, Henningsen AA, Loft A, Malchau SS, Forman J and Andersen AN. Large baby syndrome in singletons born after frozen embryo transfer (FET): is it due to maternal factors or the cryotechnique? Human reproduction $2014 ; 29: 618-627$.

Pinborg A, Wennerholm UB, Romundstad LB, Loft A, Aittomaki K, Soderstrom-Anttila V, Nygren KG, Hazekamp J and Bergh C. Why do singletons conceived after assisted reproduction technology have adverse perinatal outcome? Systematic review and meta-analysis. Human reproduction update 2013; 19:87-104. 
Rexhaj E, Paoloni-Giacobino A, Rimoldi SF, Fuster DG, Anderegg M, Somm E, Bouillet E, Allemann Y, Sartori C and Scherrer $U$. Mice generated by in vitro fertilization exhibit vascular dysfunction and shortened life span. The Journal of clinical investigation 2013; 123:5052-5060.

Romundstad LB, Romundstad PR, Sunde A, von During V, Skjaerven R, Gunnell D and Vatten LJ. Effects of technology or maternal factors on perinatal outcome after assisted fertilisation: a population-based cohort study. Lancet 2008; 372:737-743.

Rooke JA, McEvoy TG, Ashworth CJ, Robinson J, Wilmut I, Young LE and Sinclair KD. Ovine fetal development is more sensitive to perturbation by the presence of serum in embryo culture before rather than after compaction. Theriogenology 2007; 67:639-647.

Roseboom TJ, Painter RC, van Abeelen AF, Veenendaal MV and de Rooij SR. Hungry in the womb: what are the consequences? Lessons from the Dutch famine. Maturitas 2011; 70:141-145.

Sazonova A, Kallen K, Thurin-Kjellberg A, Wennerholm UB and Bergh C. Obstetric outcome in singletons after in vitro fertilization with cryopreserved/thawed embryos. Human reproduction 2012; 27:1343-1350.

Scherrer U, Rimoldi SF, Rexhaj E, Stuber T, Duplain H, Garcin S, de Marchi SF, Nicod P, Germond M, Allemann Y et al. Systemic and pulmonary vascular dysfunction in children conceived by assisted reproductive technologies. Circulation 2012; 125:1890-1896.

Schieve LA, Meikle SF, Ferre C, Peterson HB, Jeng G and Wilcox LS. Low and very low birth weight in infants conceived with use of assisted reproductive technology. The New England journal of medicine 2002; 346:731-737.

Schwarzer C, Esteves TC, Arauzo-Bravo MJ, Le Gac S, Nordhoff V, Schlatt S and Boiani M. ART culture conditions change the probability of mouse embryo gestation through defined cellular and molecular responses. Human reproduction 2012; 27:2627-2640.

Scott KA, Yamazaki Y, Yamamoto M, Lin Y, Melhorn SJ, Krause EG, Woods SC, Yanagimachi R, Sakai RR and Tamashiro KL. Glucose parameters are altered in mouse offspring produced by assisted reproductive technologies and somatic cell nuclear transfer. Biol Reprod 2010; 83:220-227.

Sinclair KD, McEvoy TG, Carolan C, Maxfield EK, Maltin CA, Young LE, Wilmut I, Robinson JJ and Broadbent PJ. Conceptus growth and development following in vitro culture of ovine embryos in media supplemented with bovine sera. Theriogenology 1998; 49:218-218.

Sinclair KD, McEvoy TG, Maxfield EK, Maltin CA, Young LE, Wilmut I, Broadbent PJ and Robinson Jj. Aberrant fetal growth and development after in vitro culture of sheep zygotes. Journal of reproduction and fertility 1999; 116:177-186.

Sjoblom C, Roberts CT, Wikland M and Robertson SA. Granulocyte-macrophage colony-stimulating factor alleviates adverse consequences of embryo culture on fetal growth trajectory and placental morphogenesis. Endocrinology 2005; 146:2142-2153.

Thompson JG, Gardner DK, Pugh PA, McMillan WH and Tervit HR. Lamb birth weight is affected by culture system utilized during in vitro pre-elongation development of ovine embryos. Biol Reprod 1995; 53:1385-1391.

Thouas GA, Jones GM and Trounson AO. The 'GO' system--a novel method of microculture for in vitro development of mouse zygotes to the blastocyst stage. Reproduction 2003; 126:161-169.

Vergouw CG, Kostelijk EH, Doejaaren E, Hompes PG, Lambalk CB and Schats R. The influence of the type of embryo culture medium on neonatal birthweight after single embryo transfer in IVF. Human reproduction 2012; 27:2619-2626.

Vermeiden JP and Bernardus RE. Are imprinting disorders more prevalent after human in vitro fertilization or intracytoplasmic sperm injection? Fertility and sterility 2013; 99:642-651.

Walker SK, Hartwich KM and Seamark RF. The production of unusually large offspring following embryo manipulation: Concepts and challenges. Theriogenology 1996; 45:111-120.

Watkins AJ, Platt D, Papenbrock T, Wilkins A, Eckert J, Kwong WY, Osmond C, Hanson M and Fleming TP. Mouse embryo culture induces changes in postnatal phenotype including raised systolic blood pressure. Proceedings 


\section{Chapter 2}

of the National Academy of Sciences of the United States of America 2007; 104:5449-5454.

Wennerholm UB, Henningsen AK, Romundstad LB, Bergh C, Pinborg A, Skjaerven R, Forman J, Gissler M, Nygren KG and Tiitinen A. Perinatal outcomes of children born after frozen-thawed embryo transfer: a Nordic cohort study from the CoNARTaS group. Human reproduction 2013; 28:2545-2553.

Wunder D, Ballabeni P, Roth-Kleiner M, Primi MP, Senn A, Chanson A, Germond M and Leyvraz C. Effect of embryo culture media on birthweight and length in singleton term infants after IVF-ICSI. Swiss medical weekly 2014 144:w14038.

www.openepi.com.

Young LE, Fernandes K, McEvoy TG, Butterwith SC, Gutierrez CG, Carolan C, Broadbent PJ, Robinson J, Wilmut I and Sinclair KD. Epigenetic change in IGF2R is associated with fetal overgrowth after sheep embryo culture. Nature genetics 2001; 27:153-154.

Zander DL, Thompson JG and Lane M. Perturbations in mouse embryo development and viability caused by ammonium are more severe after exposure at the cleavage stages. Biol Reprod 2006; 74:288-294.

Zegers-Hochschild F, Masoli D, Schwarze JE, laconelli A, Borges E and Pacheco IM. Reproductive performance in oocyte donors and their recipients: comparative analysis from implantation to birth and lactation. Fertility and sterility 2010; 93:2210-2215.

Zhan QT, Pan PP, Xu XR, Lou HY, Lou YY and Jin F. An overview of studies on psychological well-being in children born following assisted reproductive technologies. Journal of Zhejiang University Science B 2013; 14:947-960.

Zhu J, Li M, Chen L, Liu P and Qiao J. The protein source in embryo culture media influences birthweight: a comparative study between G1 v5 and G1-PLUS v5. Human reproduction 2014; 29:1387-1392.

Zhu J, Lin S, Li M, Chen L, Lian Y, Liu P and Qiao J. Effect of in vitro culture period on birthweight of singleton newborns. Human reproduction 2014; 29:448-454.

Ziebe S, Loft A, Povlsen BB, Erb K, Agerholm I, Aasted M, Gabrielsen A, Hnida C, Zobel DP, Munding B et al. A randomized clinical trial to evaluate the effect of granulocyte-macrophage colony-stimulating factor (GM-CSF) in embryo culture medium for in vitro fertilization. Fertility and sterility 2013; 99:1600-1609. 



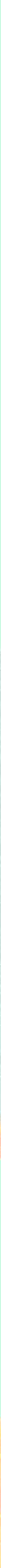




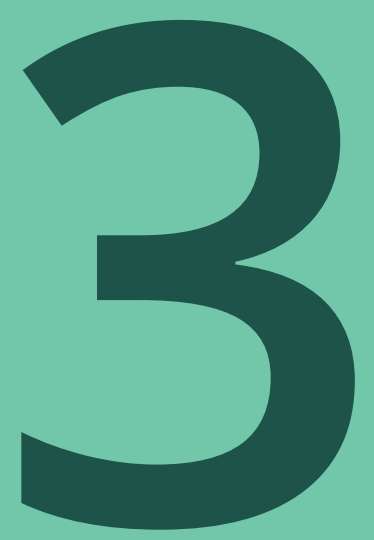

\section{IVF culture medium still affects weight and body composition of child at age 9}

Zandstra H, Spauwen B, Brentjens LBPM, Smits LJM, van der Hoeven MAHBM, van Golde RJT, Evers JLH, Dumoulin JCM, van Montfoort APA

Chapters 3 and 4 published in combination: "Association of culture medium with growth, weight and cardiovascular development of IVF children at the age of 9 years." Human Reproduction, $2018 \operatorname{Sep}$ 1;33(9):1645-1656 


\section{ABSTRACT}

\section{Study Question}

Is embryo culture media used during an IVF/ICSI treatment associated with growth and body composition of 9-year-old singleton IVF children?

\section{Summary Answer}

The choice of in vitro culture medium for human embryos is associated with differences in body weight, BMI, truncal adiposity, waist circumference and waist/hip ratio at the age of 9.

\section{What is known already?}

After alternating assignment to embryo culture in either K-SICM (Cook) or G1 $1^{\text {TM }}$ Version3 (Vitrolife), birthweight of the resulting children was significantly higher in the Vitrolife group compared to the Cook group. This weight difference persisted during the first 2 years of life (Kleijkers et al. 2014).

\section{Study Design, Size and Duration}

In this observational cohort follow-up study (MEDIUM-KIDS), parents of all singletons from a previous study were approached after the ninth birthday of their child to participate in a follow up study. The singletons were born after fresh embryo transfer of cleavage stage embryos resulting from an IVF/ICSI treatment performed between Juli 2003 and December 2006 in our clinic, when two different culture media were used alternately: either either K-SICM (Cook) or G1 TiM Version3 (Vitrolife). Follow-up measurements were performed between March 2014 and December 2016.

\section{Participants/Materials, Settings and Methods}

Parents were invited to attend our clinic with their child for a single visit lasting approximately 2.5 hours. Two experienced clinicians performed the anthropometric measurements as a part of the MEDIUM-KIDS study. Two experienced clinicians performed the following anthropometric measurements in a standardized way: height and weight of the child using calibrated scales, 4-point skinfold thickness measurements in threefold and waist and hip circumference. Data (mean \pm SD) were compared using the Student's t-test and effects of confounders were analyzed using multivariable regression analysis. 


\section{Main results and the role of chance}

Of the 294 eligible children (168 Vitrolife and 126 Cook), 136 children (75 Vitrolife and 61 Cook) participated in the study. Baseline characteristics between the participating children from the Vitrolife and Cook group were similar (mean age 9.5 years in both groups). Birthweight was higher in the Vitrolife group, in keeping with the full cohort. After correction for confounders, the difference in weight and BMI attributable to culture medium was $1.58 \mathrm{~kg}$ [95\% Cl 0.01 to 3.14$]$ and $0.84 \mathrm{~kg} / \mathrm{m}^{2}[95 \% \mathrm{Cl} 0.02$ to 1.67$]$ respectively with the Vitrolife children being heavier. Height and height corrected for age and gender (SDS scores) were similar in both groups. Furthermore, waist circumference was significantly higher in the Vitrolife group with a corrected difference of $3.21 \mathrm{~cm}$ [95\% Cl 0.60 to 5.81 leading to a 0.03 increase [95\% Cl 0.01 to 0.05] in waist/hip ratio. Subscapular skinfolds combined with suprailiacal skinfolds, (defined as truncal adiposity) was also significantly higher in Vitrolife children (adjusted difference $3.44 \mathrm{~cm}$ [95\% Cl 0.27 to 6.62]).

\section{Limitations, reasons for caution}

A limitation of the original study was its pseudo-randomised design. This and the dwindling enthusiasm of families for participation (47.7\% after 9 years) prevent us from drawing robust causal conclusions from the observed association. Nevertheless, to date this is oldest cohort of IVF/ICSI children where culture medium was allocated alternatingly and used in a blinded setting, to be studied. We believe that our participants are representative for the full cohort.

\section{Wider implications of the findings}

This study underlines the importance of structured follow-up of IVF/ICSI children to further elucidate possible long-term health effects. Health professionals and culture medium manufacturers should be aware that small changes in culture conditions and culture medium composition for the early embryo can have long-term health effects. Prolonged follow-up and structured investigations up until adulthood are necessary to gain more insight and reassurance in the cardiovascular development of IVF offspring, although longterm follow-up will become more complicated by confounding life-style and environmental factors possibly influencing development. 


\section{INTRODUCTION}

There is growing evidence that culture medium can affect birthweight of children born after ART (Zandstra et al., 2015). Although not all media comparisons lead to differences in birthweight (Lin et al., 2013, Vergouw et al., 2012), several studies found significant effects of culture medium on birthweight (Dumoulin et al., 2010, Eskild et al., 2013, Zhu et al., 2014). In 2010, we published the results of our study where a corrected difference of 112 grams in birthweight was observed in children born after IVF/ICSI, if two different culture media were used alternatingly (Dumoulin et al., 2010). In 2016, we published the results of a multicenter randomized controlled trial, where we confirmed the potential of culture medium to affect birthweight. Children born after embryo culture in Vitrolife G5 culture medium weighted 158 grams less if compared to children born after culture in human tubal fluid (HTF, Quinn) culture medium, which was a significant difference (Kleijkers et al., 2016).

Children born after IVF/ICSI are known to have an increased risk of adverse perinatal outcomes, such as low birthweight, compared to children born after natural conception (Bergh and Wennerholm, 2012, Jackson et al., 2004, Pinborg et al., 2013). Lower birthweight as a marker of poor prenatal circumstances such as poor nutrition, is related to a higher risk for non-communicable diseases in adult life like obesity (Barker, 1997, Barker, 2000). This effect is continuous, meaning that the risk increase covers the whole spectrum of birthweight. The exposure to an adverse environment in utero may induce structural and functional adaptations that can have an effect later in life. Whereas the fetal period is a critically sensitive time, it is most likely that the embryonic period is at least as precarious.

However, studies on long-term effects on health and development of IVF children are still scarce. The only study reporting on effects of culture medium on postnatal growth is a follow-up study of our first cohort, showing that the differences in birthweight persisted at least up to 2 years of age (Kleijkers et al., 2014). Some studies compared birthweight and weight during childhood between IVF/ICSI children and naturally conceived controls and found that the birthweight difference (lower in the IVF/ICSI children) disappeared at 4-5 years depending on the follow-up time. This is suggestive of catch up growth (Basatemur et al., 2010, Bay et al., 2014, Woldringh et al., 2011). In addition, also longer-term effects are reported with children born after IVF/ICSI showing more peripheral fat and an altered body composition compared to controls (Ceelen et al., 2007, Ceelen et al., 2008). This might be a gender specific effect, as Belva et al. found similar results only in girls and not in boys born after ICSI at 14 years of age (Belva et al., 2012). It is still not clear which part of the IVF treatment is responsible for the observed differences in postnatal development. 
In our review on the association of culture medium and birthweight, published in 2015, we elaborate on other possible causes for the observed differences in birthweight, including patient characteristics, fresh versus frozen embryo transfer and stimulation protocols (Zandstra et al., 2015).

Further follow-up of our initial cohort showed that the growth difference between the two culture medium groups was already present at a gestational age of 20 weeks (Nelissen et al., 2013). Evaluating possible association of culture medium with growth in human is extremely valuable, since knowledge about effects of culture conditions on long-term health of children born after IVF/ICSI is scarce. In this study, we investigated the long-term effects of culture medium on growth and body composition of 9-year-old children born after IVF/ICSI by extending the follow-up period of the children from our before mentioned culture medium study. 


\section{MATERIALS AND METHOD}

\section{Study Design}

In this prospective observational cohort study, parents of all singletons from our first culture medium study (Dumoulin et al., 2010) were approached, after the ninth birthday of their child, to participate in a follow-up study (MEDIUM-KIDS). Children resulting from a twin pregnancy were excluded from the study. It concerned all singletons born after fresh embryo transfer of cleavage stage embryos resulting from an IVF/ICSI treatment in our laboratory between July 2003-December 2006, when two culture media (either G1 version 3 (Vitrolife AB, Goteborg, Sweden) or K-SICM (Cook Medical, Brisbane, Australia) were used in alternate IVF/ICSI attempts. This protocol was strictly adhered to. The media from both suppliers were ready to use and supplemented with $5 \mathrm{mg} / \mathrm{ml}$ human serum albumin. Except for the media, all other IVF procedures (clinical as well as laboratory) were similar in both groups. Detailed information on ovarian stimulation, fertilization, culture and embryo characteristics has been described previously (Dumoulin et al., 2010).

\section{Ethical Approval}

The study was registered in the Dutch Trial register (NTR4220) and the local ethics committee approved the study. Both parents of all children gave written informed consent.

\section{Setting}

All follow-up measurements were performed between March 2014 and December 2016. Parents and their child were invited to attend our clinic for a single visit, lasting approximately 2.5 hours. Two experienced clinicians (H.Z. or B.S.) performed the measurements as a part of the MEDIUM-KIDS study for which also several cardiovascular parameters (data presented in a companion paper) were collected. The researchers as well as the children and their parents were blinded for the culture medium group the child had originally belonged to. Information on possibly confounding factors, such as lifestyle, demographic data and medical history of the child and parents were recorded by means of a questionnaire, completed by the parents at the day of examination.

\section{Outcomes}

\section{Growth}

Height (to the nearest $0.1 \mathrm{~cm}$ ) and body weight (to the nearest $0.1 \mathrm{~kg}$ ) were measured using a stadiometer (DGI 250D, The Netherlands) and scale (Seca 704, Germany) with calibrated electronic scale, while the children were dressed in light trousers and undershirt. From these measurements, BMI was calculated. Height, weight and BMI of the children were compared with the general Dutch population by calculating Standard Deviation Scores (SDS). These scores are calculated based on the value of the individual child, the 
mean value of a reference population of children of the same age and gender and the standard deviation (SD) of the reference population (https://groeiweb.pgdata.nl/calculator. asp). Reference values for height are derived from the Dutch fifth National Growth Study (2009) in which growth of 12,005 Dutch children aged 0 to 21 years was measured (Schonbeck et al., 2013). SD scores for predicted height (target height SDS) of the children were calculated based on the height of both parents. The reference values for weight and BMI are based on normal weight children from the Dutch $3^{\text {rd }}$ National Growth study in 1980 (Schönbeck et al., 2011). For classification of being overweight or obese, the International Obesity Task Force (IOTF) cut-off values were used (Cole and Lobstein, 2012).

\section{Body composition}

Skinfold thickness measurements (triceps, biceps, subscapular and suprailiac) were measured in triplicate on the non-dominant side of the body using a Harpenden skinfold caliper (Baty International, United Kingdom). The sum of the four averaged skinfold thicknesses reflects total adiposity. The sum of triceps and biceps skinfolds indicates peripheral adiposity and the sum of the subscapular and suprailiac skinfold is an index for truncal adiposity. The subscapular to triceps skinfold ratio is used as a measure of truncal to peripheral adiposity. Waist and hip circumference were measured using a calibrated tape measure and a waist/hip ratio was calculated.

\section{Statistical Analysis}

All analyses were conducted in SPSS version 24.0 (IBM-SPSS, Chicago, IL, USA). Histograms, normal Q-Q plots, and box plots were used to visualize distribution of the data. Because all data were within 4SDs from the mean, the linear regression was considered suitable for between-group comparison of continuous variables (Skovlund and Fenstad, 2001). Differences in categorical outcomes were analyzed using logistic regression. Possible confounders (mode of conception (IVF/ICSI), gender of the child, amount of physical exercise of the child and the parents, weight, (duration of) breastfeeding, height and BMI of the parents, level of education of the parents, smoking behavior of the parents, parity of the mother, family history of cardiac disease, diabetes or hypertension) were controlled for by use of multivariable linear regression. For each outcome measure, only confounders that altered the beta for culture medium $>10 \%$ in a bivariable analysis were retained and introduced in the multivariable model. In all cases, P-values represent two-sided P-values after correction for confounders using multivariable regression analysis. Testing for homogeneity of variances was performed using Levene's test of equality. Differences in baseline characteristics between the participants and non-participants were analyzed using a logistic regression analysis. P-values of $<0.05$ were considered to reflect statistical significance. 


\section{RESULTS}

A total of 294 singletons were eligible to participate in the study, 126 children born after embryo culture in Cook medium and 168 after culture in Vitrolife medium. Out of the 294 parent couples, 9 were lost to follow-up because of living abroad or failure to trace them. Of the remaining 285, a total of 136 (47.7\%) parent couples agreed for their child to participate and signed written informed consent; 61 (50.8\%) in the Cook group, and 75 (45.5\%) in the Vitrolife group ( $P=0.523)$. The baseline characteristics of the participating children were comparable to those of the children not participating in the study (Table I). Neonatal outcome and baseline characteristics of the participating children were compared, showing a similar difference in birthweight in keeping with the full cohort (Table II). We also checked for the presence of major or minor congenital abnormalities. A major congenital abnormality was reported in only 2 children (1 mild hip dysplasia and 1 unilateral duplicated ureter). Baseline characteristics of the parents were compared for the two groups (Table III). Some small differences were seen in level of education, smoking behavior, weight and height of the parents. If necessary according to the bivariable analysis, these possible confounders were included in the multivariable analysis.

Age at the day of measurement was 9.5 years (range 9.1 to 10.3 years) for the Cook children and 9.5 years (range 9.1 to 10.5) for the Vitrolife children. Height, weight and BMI outcomes at 9 years are presented in Table IV. Although height was similar between the two groups, weight and BMI and their values corrected for age and gender (SDS) differed significantly. SD scores for height in both Cook (-0.28, SD 1.20) and Vitrolife (-0.22, SD 0.86) children were close to the target height SDS based on the height of their parents (target height SDS -0.29, SD 0.62 for Cook and -0.16, SD 0.61 for Vitrolife). Weight and BMI of the Cook children was significantly lower than the average weight and BMI of the Vitrolife children. The distribution of values of these outcomes is shown in Figure 1. Multivariable regression analysis with correction for potential confounders showed a significant association between culture medium and weight. The adjusted difference in weight and BMI associated with culture medium was $1.58 \mathrm{~kg}$ [95\% Cl 0.01 to 3.14$]$ and $0.84 \mathrm{~kg} / \mathrm{m}^{2}[95 \% \mathrm{Cl}$ 0.02 to 1.67 ] respectively with Vitrolife children being heavier. Based on the IOTF criteria for overweight, $6.6 \%$ of the Cook children were classified as being overweight, compared to $18 \%$ of the Vitrolife children, however this difference was not significant $(P=0.095)$. In the Vitrolife group all four skinfolds were thicker although none statistically significant (Table V). Truncal adiposity was significantly higher in the Vitrolife group with an adjusted difference of $3.44 \mathrm{~cm}$ [95\% Cl 0.27 to 6.62$]$. In line with these findings, the adjusted waist circumference was $3.21 \mathrm{~cm}$ [95\% Cl 0.60 to 5.81$]$ higher in Vitrolife than in Cook, leading to a 0.03 increase $[95 \% \mathrm{Cl} 0.01$ to 0.05$]$ in waist/hip ratio. Results are presented in Table 
IV and Table $V$. When analyzing boys and girls separately to rule out possible effects of impending puberty, comparable results were obtained. Results are not presented, as sample sizes are small.

Table I - Parental baseline characteristics participants versus non-participants

\begin{tabular}{|c|c|c|c|c|}
\hline Characteristics & $\begin{array}{l}\text { Participants } \\
(n=136)\end{array}$ & $\begin{array}{l}\text { Non-participants } \\
(n=158)\end{array}$ & OR $(95 \% \mathrm{Cl})^{\mathrm{b}}$ & P-value ${ }^{b}$ \\
\hline Duration of infertility (yr) & $3.44(1.84)$ & $3.38(1.78)$ & $1.02(0.89-1.18)$ & 0.742 \\
\hline $\begin{array}{l}\text { Type of treatment } \\
\text { - ICSI } \\
\text { - IVF }\end{array}$ & $\begin{array}{l}81(64.3 \%) \\
45(35.7 \%)\end{array}$ & $\begin{array}{l}109(64.9 \%) \\
59(35.1 \%)\end{array}$ & $0.68(0.04-12.31)$ & 0.762 \\
\hline \multicolumn{5}{|c|}{ Maternal characteristics (at ovum pick up) } \\
\hline - Age (yr) & $32.9(3.7)$ & $32.1(3.9)$ & $1.10(1.02-1.19)$ & 0.017 \\
\hline - Height (cm) & $168.7(6.5)$ & $168.5(6.6)$ & $1.09(0.96-1.24)$ & 0.183 \\
\hline - Weight (kg) & $69.6(10.3)$ & $68.1(10.5)$ & $0.90(0.77-1.05)$ & 0.177 \\
\hline - $\mathrm{BMI}(\mathrm{kg} / \mathrm{m} 2)$ & $24.4(3.2)$ & $23.8(3.3)$ & $1.44(0.92-2.26)$ & 0.114 \\
\hline \multicolumn{5}{|c|}{ Paternal characteristics (at ovum pick up) } \\
\hline - Age (yr) & $35.6(5.0)$ & $35.5(5.7)$ & $0.98(0.93-1.03)$ & 0.462 \\
\hline - Height (cm) & $181.9(8.0)$ & $181.3(7.4)$ & $1.02(0.96-1.08)$ & 0.456 \\
\hline - Weight (kg) & $85.8(12.4)$ & $84.0(13.2)$ & $0.99(0.93-1.04)$ & 0.595 \\
\hline - $\mathrm{BMI}(\mathrm{kg} / \mathrm{m} 2)$ & $25.9(3.0)$ & $25.3(4.5)$ & $1.09(0.91-1.29)$ & 0.358 \\
\hline \multicolumn{5}{|l|}{ Neonatal characteristics } \\
\hline Gestational age (wk) & $39.6(1.7)$ & $39.2(3.7)$ & $1.08(0.96-1.21)$ & 0.222 \\
\hline Birthweight (g) & $3382.0(511.0)$ & $3336.6(619.7)$ & $1.00(1.00-1.00)$ & 0.908 \\
\hline Gender & & & $1.00(0.61-1.63)$ & 0.993 \\
\hline - Boy & $65(47.8 \%)$ & $76(48.1 \%)$ & & \\
\hline - Girl & $71(52.2 \%)$ & $82(51.9 \%)$ & & \\
\hline
\end{tabular}

a. Data presented as mean (SD) and N(\%)

b. Expected Beta $(O R)$ and P-value calculated by logistic regression analysis with "participated" as outcome variable. 
Table II - Characteristics of participating children

\begin{tabular}{lll}
\hline Characteristics & Cook (n=61) (ab) & Vitrolife (n=75) (ab) \\
\hline Boys (n) & $32(53 \%)$ & $33(44 \%)$ \\
Method of conception & & \\
- ICSI & $37(61 \%)$ & $25(67 \%)$ \\
- IVF & $24(39 \%)$ & \\
Embryotransfer & & $38(51 \%)$ \\
- SET & $35(57 \%)$ & $37(49 \%)$ \\
- DET & $26(43 \%)$ & $39.6(1.4)$ \\
Gestational age at birth (weeks) & $39.6(2.0)$ & $3(4 \%)$ \\
- Preterm birth (<37 weeks) & $2(3 \%)$ & $0(0 \%)$ \\
- Very preterm birth (<32 weeks) & $1(2 \%)$ & $3440.0(510)$ \\
Birthweight (grams) & $3310.7(506)$ & $1(1 \%)$ \\
- Low birthweight (<2500 g) & $4(7 \%)$ & $0(0 \%)$ \\
- Very low birthweight (<1500 g) & $1(2 \%)$ & $42(56 \%)$ \\
Breastfeeding (Yes) & $35(57 \%)$ & $3.6(7.6)$ \\
- Duration of breastfeeding (months) & $69(92 \%)$ \\
Performing sports (9 years of age) & $2.4(3.2)$ & $2.9(2.0)$ \\
- Duration of sports/ week (hrs) & $54(89 \%)$ & $6(8 \%)$ \\
Medical history (9 years of age) & $2.6(1.8)$ & $11(5 \%)$ \\
- Autism related disorders & & \\
- Allergic problems (including asthma, eczema) & $5(8 \%)$ & \\
\hline a. Categorical data presented as n(\%) = Number of cases (percentage of cases) & \\
b. Continuous data presented as mean (SD) & &
\end{tabular}

Table III - Parental baseline characteristics of parents of participating children

\begin{tabular}{|c|c|c|}
\hline Characteristics & Cook $(n=61)^{(b)}$ & Vitrolife $(n=75)^{(b)}$ \\
\hline \multicolumn{3}{|l|}{ Maternal characteristics } \\
\hline - Age (years) & $42.8(3.3)$ & $42.5(4.1)$ \\
\hline - Height (cm) & $168.1(7.1)$ & $169.3(6.2)$ \\
\hline - Weight (kg) & $70.6(11.7)$ & $73.2(6.2)$ \\
\hline - $\mathrm{BMI}\left(\mathrm{kg} / \mathrm{m}^{2}\right)$ & $24.9(3.7)$ & $25.5(3.8)$ \\
\hline - Smoking $\geq 10$ cigarettes/day & $11(18 \%)$ & $7(9 \%)$ \\
\hline - Higher Education & $28(46 \%)$ & $22(29 \%)$ \\
\hline - Caucasian ethnicity & $57(93 \%)$ & $70(93 \%)$ \\
\hline - Exercising regularly & $35(57 \%)$ & $43(57 \%)$ \\
\hline - Primary infertility & $47(77 \%)$ & $58(77 \%)$ \\
\hline \multicolumn{3}{|l|}{ Paternal characteristics } \\
\hline - Age (years) & $45.7(5.4)$ & $45.0(4.6)$ \\
\hline - Height (cm) & $180.9(7.9)$ & $182.5(8.0)$ \\
\hline - Weight (kg) & $86.7(11.8)$ & $90.5(13.9)$ \\
\hline - $\mathrm{BMI}\left(\mathrm{kg} / \mathrm{m}^{2}\right)$ & $26.5(3.1)$ & $27.1(3.4)$ \\
\hline - Smoking $\geq 10$ cigarettes/day & $6(10 \%)$ & $12(16 \%)$ \\
\hline - Higher Education & $21(34 \%)$ & $34(45 \%)$ \\
\hline - Caucasian ethnicity & $59(97 \%)$ & $72(96 \%)$ \\
\hline - Exercising regularly & $34(56 \%)$ & $39(52 \%)$ \\
\hline
\end{tabular}


Table IV - Anthropometrics at the day of measurement

\begin{tabular}{|c|c|c|c|c|}
\hline & Cook $(n=61)^{(a)}$ & Vitrolife $(n=75)^{(a)}$ & Adj.Beta (bc) & P-value $(b c)$ \\
\hline Height $[\mathrm{cm}]^{(\mathrm{ci})}$ & $138.8(7.8)$ & $139.3(6.0)$ & $-0.38[-2.38$ to 1.62$]$ & 0.705 \\
\hline SDS ${ }^{d}$ Height (ci) & $-0.28(1.2)$ & $-0.22(0.86)$ & $-0.08[-0.37$ to 0.22$]$ & 0.614 \\
\hline Weight [Kg] ${ }^{(\mathrm{cii})}$ & $32.1(5.9)$ & $34.2(6.6)$ & $1.58[0.01$ to 3.14$]$ & $0.049 *$ \\
\hline SDS $^{d}$ Weight ${ }^{\text {(cii) }}$ & $0.11(1.2)$ & $0.57(1.0)$ & 0.40 [0.11 to 0.69] & $0.007^{\star}$ \\
\hline BMI $\left[\mathrm{kg} / \mathrm{m}^{2}\right]^{\text {(ciii) }}$ & $16.6(2.3)$ & $17.5(2.5)$ & 0.84 [0.02 to 1.67] & $0.046 *$ \\
\hline SDS $^{d} B M I^{\text {(ciii) }}$ & $0.17(1.2)$ & $0.67(1.2)$ & $0.47[0.07$ to 0.86$]$ & 0.021 * \\
\hline
\end{tabular}

a) Data presented as mean (SD)

b) Adjusted beta reflects the effect size and direction of the association of culture medium with the parameter [95\% Cl]

c) Adjusted using a multivariable regression model for potential confounders having a $>10 \%$ effect on adjusted Beta for culture medium in a bivariable model.

i. Height of the mother, height of the father, (duration of) breastfeeding, level of education of the mother

ii. Smoking behaviour of the mother, level of education of the mother, height at the day of measurement

iii. Smoking behaviour of mother and father, level of education of the mother and father

d) SDS = Standard Deviation Score (number of population standard deviations an individual differs from the population mean)

- Indicates a significant association with $P<0.05$

Table V - Skinfold thickness and hip/waist circumference

\begin{tabular}{|c|c|c|c|c|}
\hline Characteristics & Cook $(n=61)^{(a)}$ & Vitrolife $(n=75)^{\text {(a) }}$ & Adj.Beta (bc) & P-Value (bc) \\
\hline \multicolumn{5}{|l|}{ Skinfold thickness [mm] } \\
\hline - Triceps ${ }^{(\mathrm{i} i)}$ & $11.1(4.4)$ & $12.1(4.6)$ & $0.50[-1.09$ to 2.09$]$ & 0.533 \\
\hline - Biceps ${ }^{(\mathrm{c} i)}$ & $7.7(3.8)$ & $9.2(4.9)$ & $0.99[-0.54$ to 2.52$]$ & 0.202 \\
\hline - Subscapular (ci) & $6.6(3.1)$ & $7.9(3.5)$ & $1.06[-0.12$ to 2.48$]$ & 0.056 \\
\hline - Suprailiac (ci) & $9.5(6.0)$ & $11.5(7.0)$ & $1.27[-1.00$ to 3.55$]$ & 0.270 \\
\hline Peripheral Adiposity [mm] ${ }^{(\mathrm{cii})}$ & $18.8(7.6)$ & $21.2(8.8)$ & $1.84[-1.00$ to 4.68$]$ & 0.201 \\
\hline Truncal Adiposity [mm] (ciii) & $16.2(8.7)$ & $19.4(9.9)$ & $3.44[0.27$ to 6.62$]$ & $0.034 *$ \\
\hline Central to peripheral adiposity (cii) & $0.62(0.20)$ & $0.72(0.48)$ & $0.08[-0.04$ to 0.22$]$ & 0.238 \\
\hline Total Adiposity [mm] (cii) & $35.0(15.4)$ & $40.7(17.6)$ & $4.70[-0.97$ to 10.38$]$ & 0.104 \\
\hline Waist [cm] (civ) & $58.0(6.1)$ & $61.4(8.4)$ & $3.21[0.60$ to 5.81$]$ & $0.016 *$ \\
\hline Hip $[\mathrm{cm}]^{(\mathrm{cv})}$ & $69.7(6.5)$ & $71.2(7.1)$ & $1.62[-0.66$ to 3.90$]$ & 0.162 \\
\hline Waist/Hip ratio (cvi) & $0.83(0.06)$ & $0.86(0.07)$ & 0.03 [0.01 to 0.05$]$ & 0.022 \\
\hline
\end{tabular}

a) Data presented as mean (SD)

b) Adjusted beta reflects the effect size and direction of the association of culture medium with the parameter [95\% CI]

c) Adjusted when necessary (>10\% effect on adjusted Beta for culture medium) for relevant confounders using a multivariable regression model

i. Weight of the father, level of education of father and mother, gender of the child, breastfeeding

ii. Weight of the father, level of education of father and mother, gender of the child, smoking behaviour of the mother, weekly amount of physical exercise

iii. Weight of the father, level of education of the mother, gender of the child, weekly amount of physical exercise

iv. Level of education of father and mother, weekly amount of physical exercise, breastfeeding

$v$. Level of education of the father, smoking behaviour of the mother, weekly amount of physical exercise, breastfeeding, weight of father and mother, height of father and mother.

vi. Gender of the child, parity of the mother, level of education of the mother

- Indicates a significant association with $P<0.05$ 


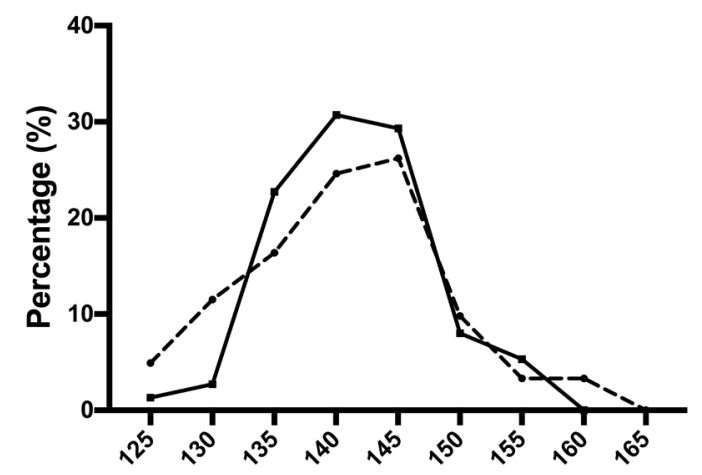

Height (cm)
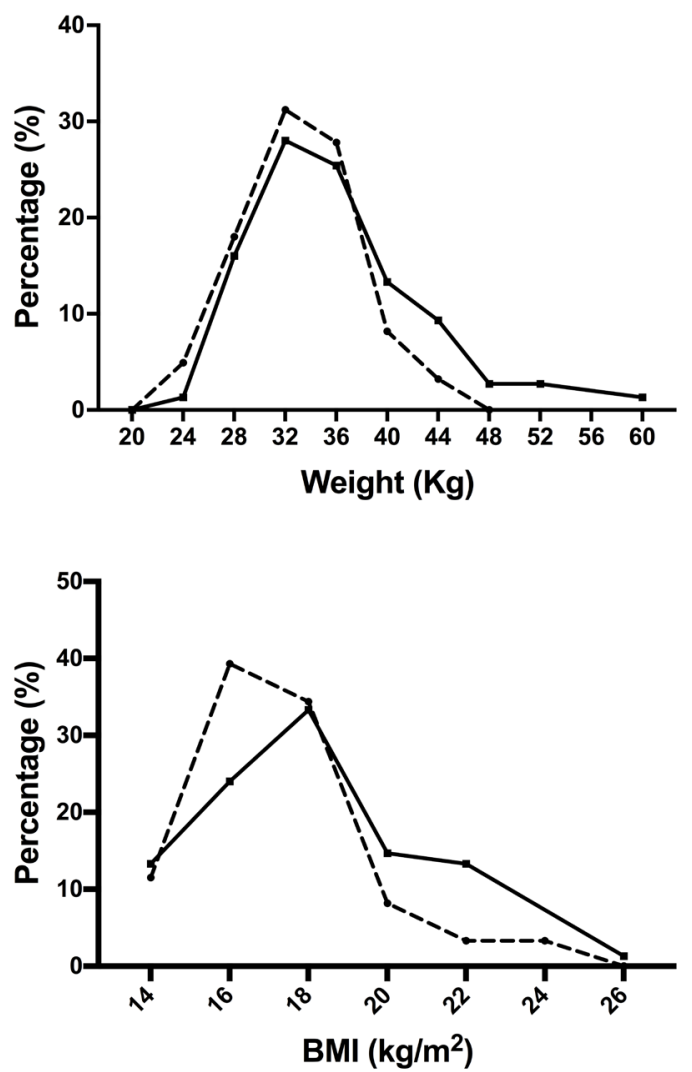

n. Cook

$\rightarrow$ Vitrolife

Cook

$\rightarrow$ Vitrolife

-a. Cook

$\rightarrow$ Vitrolife

The dashed line represents the distribution for the Cook children and the continuous line represents the distribution for the Vitrolife children. 


\section{DISCUSSION}

The results of our study suggest that culture medium affects weight, BMI and body composition of IVF children at the age of nine years. This includes waist circumference, waist/ hip ratio and truncal adiposity. After correction for confounders, the association with culture medium persists. The difference in height, present at 2 years of age (Kleijkers et al., 2014) was no longer present at 9 years, which indicates different growth trajectories for the two groups.

Could it be possible to relate the differences found in anthropometric parameters between both media groups to differences in the composition of the media used in our study? It is important to realize that both types of media have not been marketed for some time and the exact composition is not publicly available. Both manufacturers provide information in their product inserts about the composition of the media in terms of which constituents are present, but do not state the exact concentration. However, this information in product inserts and certificates of analysis, as well as chemical analysis that were performed in our hospital at that time indicate that there may be both similarities and differences. Both media are so-called sequential media types designed specifically for IVF and embryo culture. The most important differences in the media compositions are the osmolality ( $254 \pm 5 \mathrm{mOsm} / \mathrm{kg}$ in the Vitrolife medium and $285-295 \pm 5 \mathrm{mOsm} / \mathrm{kg}$ in the Cook medium), the presence of hyaluronan only in the Vitrolife medium, glucose content $(0.5 \mathrm{mM} / \mathrm{L}$ in the Vitrolife medium and no glucose at all in the Cook medium), and different lactate and pyruvate content resulting in a higher lactate/pyruvate ratio in the Vitrolife medium as compared to the Cook medium. It has been shown that changing the lactate/pyruvate ratio in the culture medium during the pronuclear stage can result in different post-natal growth rates in the mouse (Banrezes et al., 2011). Also the type of glutamine differed between the two culture media. The version of the Cook medium that was used in our study in the period of 2003 to 2006 contained, according to the product insert, the free L-glutamine which is known to decompose spontaneously, releasing ammonium, as opposed to Vitrolife medium that contained the more stable dipeptide alanyl-glutamine (Kleijkers et al., 2016). Several studies in animal models have shown that ammonium has a negative effect on fetal development (Zander et al., 2006). Finally, the media from both suppliers were supplemented with $5 \mathrm{mg} / \mathrm{ml}$ human serum albumin. This is important, since it is known that different protein supplements used for human clinical embryo culture can possibly influence embryo development mice (Morbeck et al., 2014). It will be clear that with all these differences between the two culture media that were used in our study, some of which have been shown to affect fetal development and even postnatal development, it is not possible to pinpoint the differences found between both media groups to specific differences in the composition of the media used in our study. 
Effects of fertility treatments on growth have been reported before. A study by Bay et al. analyzed birthweight and weight at the age of 5 years in children born after fertility treatment and found a significantly lower birthweight in the children born after ART if compared to natural conception (Bay et al., 2014). However at the age of 5 years no significant differences were observed between the groups. Another study by Basatemur et al. did not find any differences in growth and weight when comparing IVF/ICSI children from birth until 12 years of age with naturally conceived matched controls (Basatemur et al., 2010).

The increased weight in the children from the Vitrolife group compared to Cook is likely to be explained by an increase in adipose tissue mass, as this group had a higher truncal adiposity and waist circumference. The group of Ceelen and co-workers reported that IVF could affect not only catch-up growth, but also body composition. They found that children born after IVF have more peripheral body fat than children from subfertile couples who conceived naturally, suggesting that body fat distribution might be disturbed not only by the patient characteristics, but also by the techniques used in IVF (Ceelen et al., 2009, Ceelen et al., 2007).

The increased weight and adiposity might indicate a very early onset of a disadvantageous cardiovascular profile, which warrants close follow-up of IVF children. Several studies have shown, that childhood overweight and obesity significantly increases the chances for Type 2 Diabetes, obesity and coronary heart disease in later life (Eriksson et al., 2001, Eriksson et al., 2003, veena et al., 2014).

Several animal studies have shown that culture conditions can affect postnatal development. In a study by Watkins et al. the effect of embryo culture and transfer was investigated by comparing postnatal growth and physiological activity in mice born after embryo culture and ovarian hyperstimulation with mice born after only ovarian hyperstimulation and embryo transfer (no embryo culture) and also with mice born after in vivo embryo development. They found minor effects of embryo culture on postnatal growth, but did find higher systolic blood pressure at 21 weeks compared with in vivo development (Watkins et al., 2007). In another study by Donjacour et al. postnatal effects of two different culture media were analyzed. If results of these mice pups are compared, the male mice showed a difference in body size and glucose intolerance between the two groups (Donjacour et al., 2014).

From studies on follow up of children born after being in the womb during the Dutch WWII famine, it was already known that intrauterine stress can alter postnatal health (Roseboom et al., 2011, Roseboom et al., 2001). Our study shows that also alterations in only the early embryonic stage can have long-lasting effects. 
The strength of our study is the alternating allocation of culture media. Although not as perfect as randomization, this is the oldest cohort of children where culture medium was allocated alternatingly and used in a blinded setting, in the same period, with all other conditions identical. Also, the current study was performed in a standardized way by two experienced investigators who were blinded - as were the children and their parents - for the culture medium group the child belonged to. However, this study also has limitations. One of the limitations is the relatively low participation rate of $47.7 \%$, although characteristics at the time of the IVF treatment of participants and non-participants were similar. Since we had no permission to contact the non-participants, it was not possible to determine whether all baseline characteristics were still the same at the time of measurement.

Our study suggests that at nine years of age, children who, as an embryo, were cultured in Vitrolife culture medium have a significantly higher weight, BMI, waist circumference, waist/hip ratio and truncal adiposity compared to the children who as an embryo were cultured in Cook culture medium. It is not yet clear which of the groups is to be considered the healthiest or which of the groups will have a greater chance of developing (cardiovascular) disease in later life. Therefore it is not possible to state which medium should be preferred. The observed differences may predispose to future (cardiovascular) health risks. This underlines the importance of structured long-term follow-up of IVF/ICSI children. 


\section{REFERENCES}

Banrezes B, Sainte-Beuve T, Canon E, Schultz RM, Cancela J, Ozil JP. Adult body weight is programmed by a redox-regulated and energy-dependent process during the pronuclear stage in mouse. PLoS One 2011;6: e29388.

Barker DJ. Maternal nutrition, fetal nutrition, and disease in later life. Nutrition 1997;13: 807-813.

Barker DJ. In utero programming of cardiovascular disease. Theriogenology 2000;53: 555-574.

Basatemur E, Shevlin M, Sutcliffe A. Growth of children conceived by IVF and ICSI up to 12years of age. Reprod Biomed Online 2010;20: 144-149.

Bay B, Mortensen EL, Kesmodel US. Is subfertility or fertility treatment associated with long-term growth in the offspring? A cohort study. Fertil Steril 2014;102: 1117-1123.

Belva F, Painter R, Bonduelle M, Roelants M, Devroey P, De Schepper J. Are ICSI adolescents at risk for increased adiposity? Hum Reprod 2012;27: 257-264

Bergh C, Wennerholm UB. Obstetric outcome and long-term follow up of children conceived through assisted reproduction. Best Pract Res Clin Obstet Gynaecol 2012;26: 841-852.

Ceelen M, van Weissenbruch MM, Prein J, Smit J, Vermeiden JP, Spreeuwenberg M, van Leeuwen FE, Delemarre-van de Waal HA. Growth during infancy and early childhood in relation to blood pressure and body fat measures at age 8-18 years of IVF children and spontaneously conceived controls born to subfertile parents. Hum Reprod 2009;24: 2788-2795.

Ceelen M, van Weissenbruch MM, Roos JC, Vermeiden JP, van Leeuwen FE, Delemarre-van de Waal HA. Body composition in children and adolescents born after in vitro fertilization or spontaneous conception. J Clin Endocrinol Metab 2007;92: 3417-3423.

Ceelen M, van Weissenbruch MM, Vermeiden JP, van Leeuwen FE, Delemarre-van de Waal HA. Growth and development of children born after in vitro fertilization. Fertil Steril 2008;90: 1662-1673.

Cole TJ, Lobstein T. Extended international (IOTF) body mass index cut-offs for thinness, overweight and obesity. Pediatr Obes 2012;7: 284-294.

Delle Piane L, Lin W, Liu X, Donjacour A, Minasi P, Revelli A, Maltepe E, Rinaudo PF. Effect of the method of conception and embryo transfer procedure on mid-gestation placenta and fetal development in an IVF mouse model. Hum Reprod 2010;25: 2039-2046.

Donjacour A, Liu X, Lin W, Simbulan R, Rinaudo PF. In vitro fertilization affects growth and glucose metabolism in a sex-specific manner in an outbred mouse model. Biol Reprod 2014;90: 80.

Dumoulin JC, Land JA, Van Montfoort AP, Nelissen EC, Coonen E, Derhaag JG, Schreurs IL, Dunselman GA, Kester $A D$, Geraedts JP et al. Effect of in vitro culture of human embryos on birthweight of newborns. Hum Reprod 2010;25: 605-612.

Eriksson J, Forsén T, Tuomilehto J, Osmond C, Barker D. Size at birth, childhood growth and obesity in adult life. Int J Obes Relat Metab Disord 2001;25: 735-740.

Eriksson JG, Forsén T, Tuomilehto J, Osmond C, Barker DJ. Early adiposity rebound in childhood and risk of Type 2 diabetes in adult life. Diabetologia 2003;46: 190-194.

Eskild A, Monkerud L, Tanbo T. Birthweight and placental weight; do changes in culture media used for IVF matter? Comparisons with spontaneous pregnancies in the corresponding time periods. Hum Reprod 2013;28: $3207-$ 3214.

Feuer S, Rinaudo P. Preimplantation stress and development. Birth Defects Res C Embryo Today 2012;96: 299-314.

Feuer S, Rinaudo P. From Embryos to Adults: A DOHaD Perspective on In Vitro Fertilization and Other Assisted Reproductive Technologies. Healthcare (Basel) 2016;4.

Fleming TP, Velazquez MA, Eckert JJ. Embryos, DOHaD and David Barker. J Dev Orig Health Dis 2015;6: 377-383. 
Jackson RA, Gibson KA, Wu YW, Croughan MS. Perinatal outcomes in singletons following in vitro fertilization: a meta-analysis. Obstet Gynecol 2004;103: 551-563.

Kleijkers SH, Mantikou E, Slappendel E, Consten D, van Echten-Arends J, Wetzels AM, van Wely M, Smits LJ, van Montfoort AP, Repping S et al. Influence of embryo culture medium (G5 and HTF) on pregnancy and perinatal outcome after IVF: a multicenter RCT. Hum Reprod 2016;31: 2219-2230.

Kleijkers SH, van Montfoort AP, Smits LJ, Viechtbauer W, Roseboom TJ, Nelissen EC, Coonen E, Derhaag JG, Bastings $L$, Schreurs IE et al. IVF culture medium affects post-natal weight in humans during the first 2 years of life. Hum Reprod 2014;29: 661-669.

Lin S, Li M, Lian Y, Chen L, Liu P. No effect of embryo culture media on birthweight and length of newborns. Hum Reprod 2013;28: 1762-1767.

Morbeck DE, Paczkowski M, Fredrickson JR, Krisher RL, Hoff HS, Baumann NA, Moyer T, Matern D. Composition of protein supplements used for human embryo culture. J Assist Reprod Genet 2014;31: 1703-1711.

Pinborg A, Wennerholm UB, Romundstad LB, Loft A, Aittomaki K, Söderström-Anttila V, Nygren KG, Hazekamp J, Bergh C. Why do singletons conceived after assisted reproduction technology have adverse perinatal outcome? Systematic review and meta-analysis. Hum Reprod Update 2013;19: 87-104.

Roseboom TJ, Painter RC, van Abeelen AF, Veenendaal MV, de Rooij SR. Hungry in the womb: what are the consequences? Lessons from the Dutch famine. Maturitas 2011;70: 141-145.

Roseboom TJ, van der Meulen JH, van Montfrans GA, Ravelli AC, Osmond C, Barker DJ, Bleker OP. Maternal nutrition during gestation and blood pressure in later life. J Hypertens 2001;19: 29-34.

Schonbeck Y, Talma H, van Dommelen P, Bakker B, Buitendijk SE, HiraSing RA, van Buuren S. The world's tallest nation has stopped growing taller: the height of Dutch children from 1955 to 2009. Pediatr Res 2013;73: 371-377.

Schönbeck Y, Talma H, van Dommelen P, Bakker B, Buitendijk SE, Hirasing RA, van Buuren S. Increase in prevalence of overweight in Dutch children and adolescents: a comparison of nationwide growth studies in 1980, 1997 and 2009. PLoS One 2011;6: e27608.

Skovlund E, Fenstad GU. Should we always choose a nonparametric test when comparing two apparently nonnormal distributions? J Clin Epidemiol 2001;54: 86-92.

Veena SR, Hegde BG, Ramachandraiah S, Krishnaveni GV, Fall CH, Srinivasan K. Relationship between adiposity and cognitive performance in 9-10-year-old children in South India. Arch Dis Child 2014;99: 126-134.

Vergouw CG, Kostelijk EH, Doejaaren E, Hompes PG, Lambalk CB, Schats R. The influence of the type of embryo culture medium on neonatal birthweight after single embryo transfer in IVF. Hum Reprod 2012;27: 2619-2626.

Watkins AJ, Platt D, Papenbrock T, Wilkins A, Eckert J, Kwong WY, Osmond C, Hanson M, Fleming TP. Mouse embryo culture induces changes in postnatal phenotype including raised systolic blood pressure. Proc Natl Acad Sci US A 2007;104: 5449-5454.

Woldringh GH, Hendriks JC, van Klingeren J, van Buuren S, Kollee LA, Zielhuis GA, Kremer JA. Weight of in vitro fertilization and intracytoplasmic sperm injection singletons in early childhood. Fertil Steril 2011;95: 2775-2777.

Zander DL, Thompson JG, Lane M. Perturbations in mouse embryo development and viability caused by ammonium are more severe after exposure at the cleavage stages. Biol Reprod 2006;74: 288-294.

Zandstra H, Van Montfoort AP, Dumoulin JC. Does the type of culture medium used influence birthweight of children born after IVF? Hum Reprod 2015;30: 2693.

Zhu J, Lin S, Li M, Chen L, Lian Y, Liu P, Qiao J. Effect of in vitro culture period on birthweight of singleton newborns. Hum Reprod 2014;29: 448-454. 


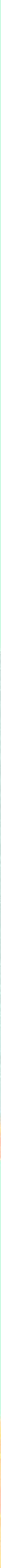




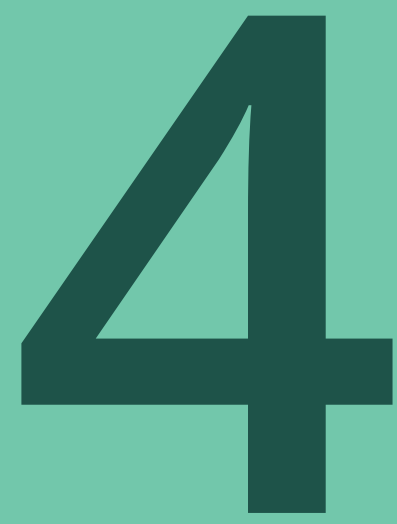

\section{Association of culture medium with cardiovascular development in 9-year-old IVF children}

Zandstra H, Spauwen B, Touwslager RNH, Bons JAP, Mulder AL, Smits LJM, van Golde RJT, Evers JLH, Dumoulin JCM, Van Montfoort APA

Chapters 3 and 4 published in combination: "Association of culture medium with growth, weight and cardiovascular development of IVF children at the age of 9 years." Human Reproduction, $2018 \operatorname{Sep~1;33(9):1645-1656~}$ 


\section{ABSTRACT}

\section{Study Question}

Is embryo culture medium associated with cardiovascular characteristics of 9-year old children born after an IVF/ICSI treatment?

\section{Summary Answer}

No significant differences were observed between the Cook and Vitrolife group for blood pressure, fasting glucose, insulin, cholesterol and TSH, nor for endothelial function.

\section{What is known already?}

Children born after IVF/ICSI are known to have an increased risk of having low birthweight which is correlated with a higher risk for cardiovascular disease (Developmental Origin of Health and Disease, DOHaD). Some studies show that IVF children exhibit a significantly higher systolic and diastolic blood pressure and higher fasting glucose levels compared to naturally conceived children. After alternating assignment to $\mathrm{G} 1^{\mathrm{TM}}$ Version3 (Vitrolife) or K-SICM (Cook) embryo culture media, birthweight of the resulting children was significantly higher in the Vitrolife group and they remained heavier during the first 2 years of life.

\section{Study Design, Size and Duration}

In this observational cohort study (MEDIUM-KIDS), parents of the singletons from our previous study were approached for further follow up after the ninth birthday of their child. The singletons were born after an IVF/ICSI treatment performed between July 2003 and December 2006 in our clinic, when two different culture media were used alternately: either G1 ${ }^{\mathrm{TM}}$ Version3 (Vitrolife) or K-SICM (Cook). Follow up measurements were performed between March 2014 and December 2016.

\section{Participant/Materials, Settings and Methods}

Parents were invited to attend our clinic with their child for a single visit lasting approximately 2.5 hours. Two experienced clinicians performed all measurements as part of the MEDIUM-KIDS study. The following cardiovascular parameters were measured in a standardized way: blood pressure, heart rate, endothelial function by skin laser-Doppler with iontophoresis using vasodilatory drugs and glycocalyx function using Glycocheck ${ }^{\mathrm{TM}}$. Cortisol and cortisone concentrations in hair were measured and compared. A blood sample was taken after an overnight fast for insulin, glucose, TSH, and lipid analysis. Blood samples of the IVF children were compared with a non-IVF control group. Data (mean \pm SD) were analyzed by Student's t-test and effects of confounders were analyzed using multivariable regression analysis. 


\section{Main Results and the role of chance}

Of the 294 eligible children, 136 children (75 Vitrolife and 61 Cook) participated in the study. Baseline characteristics between the participating children from the Vitrolife and Cook group were similar. Birthweight was higher in the Vitrolife group, but not significantly due to the smaller sample size. Both systolic (adj. beta 0.364 [95\% Cl -2.129 to 2.856],) and diastolic (adj. beta 0.275 [95\% Cl -2.105 to 2.654]) blood pressures ( $\mathrm{mmHg}$ ) were comparable for the two groups. After an overnight fast, cholesterol, glucose, insulin, low and high-density lipoprotein (LDL, HDL), triglycerides and TSH were normal and similar in the two groups. Endothelial function in the microcirculation was compared by using maximum perfusion units corrected for the baseline value as a measure for vasodilatory capacity. There were no significant differences between the two groups. Cortisol and cortisone concentration in hair samples were comparable.

\section{Limitations, reasons for caution.}

The most important limitation of this study is the relatively low participation rate, however no differences were seen in baseline characteristics between participants and non-participants. Nevertheless, to date this is oldest cohort of IVF/ICSI children where culture medium was allocated alternatingly and used in a blinded setting, to be studied. We believe that our participants are representative for the full cohort. The current number of participants was sufficient to rule out differences as little as $3 \mathrm{mmHg}$ in systolic and diastolic blood pressures.

\section{Wider implications of the findings}

The similar cardiovascular results for the two groups are reassuring but the children may still be too young to detect differences in cardiovascular development. Prolonged follow-up and structured investigations up until adulthood are necessary to gain more insight and reassurance in the cardiovascular development of IVF offspring, although longterm follow-up will become more complicated by confounding life-style and environmental factors possibly influencing development. 


\section{INTRODUCTION}

Children born after IVF/ICSI are known to have an increased risk of adverse perinatal outcomes, such as low birthweight, compared to children born after natural conception (Bergh and Wennerholm, 2012, Jackson et al., 2004, Pinborg et al., 2013). Barker et al. found that birthweight is strongly and inversely associated with the risk of cardiovascular diseases and diabetes at adult age (Barker, 2006). The Barker Hypothesis was further developed into the Developmental Origin of Health and Disease (DOHaD) hypothesis, which states that lower birthweight and poor perinatal outcome can affect postnatal growth, glucose metabolism, fat distribution and vascular function (Barker, 1999, Feuer and Rinaudo, 2012). This relation can be partly explained by altered (epigenetic) programming of the fetus in utero due to nutritional, hormonal or metabolic changes in the uterine environment that both affect fetal growth and susceptibility for disease in later life. Such adaptive developmental plasticity gives advantages in environments that change over several generations. However when environmental conditions during development change too much between conception and adulthood, a substantial mismatch will contribute to disease risk (Gluckman et al., 2009). The exposure to an adverse environment may induce structural and functional adaptations that have an effect later in life. In vitro culture of the embryos, as part of the IVF procedure, might influence developmental programming during a very early stage of development (Market-Velker et al., 2010, Zandstra et al., 2015).

In humans, research on long term health effects of in vitro culture is still scarce. The general health outcome as well as cognitive development of children born as a result of IVF treatment is positive (Barbuscia and Mills, 2017, Hart and Norman, 2013). However, several studies compared IVF children with naturally conceived controls and found that, besides lower birthweight, IVF children also had higher systolic and diastolic blood pressure levels, higher fasting glucose levels, a higher percentage of peripheral body fat and altered endothelial function (Belva et al., 2007, Ceelen et al., 2009, Ceelen et al., 2007, Chen et al., 2014, Sakka et al., 2010, Scherrer et al., 2012). It is still not clear which part of the IVF treatment is responsible for the observed differences in postnatal development.

In animal studies it has been reported that culture media can irreversibly affect long-term postnatal development, growth and physiology with changes in blood pressure, glucose metabolism and altered behaviour, besides significant changes in birthweight (Feuer et al., 2014, Watkins et al., 2007). A study by Fernandez-Gonzalez and co-workers reported that mRNA expression of some imprinted genes was significantly affected in blastocysts cultured in the presence of 10\% FCS. These results raise concerns and might indicate that long-term postnatal development, growth, and physiology can be altered during the 
preimplantation period of embryo development by suboptimal in vitro culture (Fernández-Gonzalez et al., 2004). Scott et al. compared the outcome of mice born after ART (IVF/ ICSI) with naturally conceived controls. They found glucose and insulin metabolism to be impaired in ART mice with significant differences in clearance of glucose after a bolus was administered. Furthermore the males required more insulin to clear the bolus (Scott et al., 2010).

In 2010, we published the results of our study where a corrected difference of 112 grams in birthweight was observed in children born after IVF/ICSI, if two different culture media were used alternatingly (Dumoulin et al., 2010). Further follow-up showed that the growth difference between the two culture medium groups was already present at a gestational age of 20 weeks (Nelissen et al., 2013) and that it was still present at the age of 2 years (Kleijkers et al., 2014). Evaluating possible effects of culture medium and the differences in birthweight on cardiovascular health is extremely valuable, since knowledge about effects of culture conditions on long-term health of children born after IVF/ICSI is scarce. The aim of our current study is to analyze and compare the cardiovascular development of the children from this cohort. 


\section{MATERIALS AND METHOD}

\section{Study design}

In this prospective observational cohort study, parents of all singletons from our first culture medium study (Dumoulin et al., 2010) were approached, after the ninth birthday of their child, to participate in a follow-up study (MEDIUM-KIDS). Children resulting from a twin pregnancy were excluded from the study. It concerned all singletons born after fresh embryo transfer of cleavage stage embryos resulting from an IVF/ ICSI treatment in our laboratory between July 2003-December 2006, when two culture media (either G1 version 3 (Vitrolife AB, Goteborg, Sweden) or K-SICM (Cook Medical, Brisbane, Australia) were used in alternate IVF/ICSI attempts. This protocol was strictly adhered to. The media from both suppliers were ready to use and supplemented with $5 \mathrm{mg} / \mathrm{ml}$ human serum albumin. Except for the media, all other IVF procedures (clinical as well as laboratory) were similar in both groups. Detailed information on ovarian stimulation, fertilization, culture and embryo characteristics has been described previously (Dumoulin et al., 2010).

\section{Ethical approval}

The study was registered in the Dutch Trial register (NTR4220) and the local ethics committee approved the study. Both parents of all children gave written informed consent.

\section{Setting}

All follow-up measurements were performed between March 2014 and December 2016. Parents and their child were invited to attend our clinic for a single visit, lasting approximately 2.5 hours. Two experienced clinicians (H.Z and B.S) performed all cardiovascular measurements as part of the MEDIUM-KIDS study for which also several anthropometric parameters (Chapter 3) were collected. The researchers as well as the children and their parents were blinded for the culture medium group the child belonged to. Information on possibly confounding factors, such as lifestyle, demographic data and medical history of the child and parents were recorded by means of a questionnaire, completed by the parents at the day of examination.

\section{Outcomes}

Blood pressure

Systolic and diastolic blood pressures as well as heart rate were measured in triplicate at the non-dominant arm while the child was in a sitting position after a minimum of 30 minutes rest (lying in a supine position on a bed). It was measured using a calibrated automatic device (Accutorr Plus, Datascope Inc., Montvale, New Jersey, USA). The mean of these three measurements was used in the analyses. For both systolic and diastolic blood 
pressure, we calculated SD-scores and percentiles to correct for age, height and gender. These were based on an American reference cohort (National, 2004).

Lipids, g/ucose and insulin level

Blood samples $(15 \mathrm{ml})$ were drawn in the morning at the start of the measurement session after an overnight fast. Fasting plasma glucose was measured using an enzymatic colorimetric assay (Cobas 8000 instrument, Roche Diagnostics, Mannheim, Germany). Fasting insulin was determined in serum using an immunometric assay (XPi instrument, Siemens Medical Solutions Diagnostics, LA). Haemoglobin A1c (HbA1c) was measured in heparin anti-coagulated whole blood by ion exchange chromatography on the BioRad Variant II instrument (Biorad Clinical Diagnostics, CA, USA). Serum thyroid stimulating hormone (TSH) was measured using an electrochemiluminescent immunometric assay (Cobas 8000 instrument, Roche Diagnostics, Mannheim, Germany). Total cholesterol, high density lipoprotein (HDL) cholesterol, and triglycerides were determined using Enzymatic colorimetric assay (Cobas 8000 instrument, Roche Diagnostics, Mannheim, Germany), low density lipoprotein (LDL) cholesterol was calculated using the Friedewald formula. To compare the blood values of the two culture media groups, a control group of healthy non-IVF children was added. Residual blood stored in our hospitals laboratory was used from children who had their blood tested for several benign reasons not related to TSH or lipid profiles. The blood was stored as surplus blood at $-80{ }^{\circ} \mathrm{C}$ and not thawed before we used it for our analysis. Since it was frozen blood it was only possible to analyse lipid profile and TSH. A computer-based selection of samples of possible eligible children of similar age as our study population (i.e. 9 years) was performed. The medical files of all possibly eligible children were manually screened by one physician (H.Z.) for suitability to be included in the control group. Children who were on medication or who were suffering from diabetes mellitus, morbid obesity, thyroid disorders, cancer, metabolic diseases or other conditions possibly influencing their metabolic profile were excluded. Data were used anonymously and only children whose parents agreed with storage and possible use of the residual blood were analysed. Included children had their blood drawn for example for allergy testing, fractured bones if surgery was needed, screening for benign cases of hair loss (alopecia) or screening for other benign conditions such as fatigue or abdominal pain without a clear cause. If the motive for the blood sample was unclear or if the medical record of the child was not available, the child was excluded.

\section{Endothelial function}

Endothelial dysfunction precedes the formation of atheromatous plaques. Evaluation of endothelial function is therefore a valuable indicator for detecting early stages of an adverse cardiovascular profile. Endothelial function was evaluated by means of two measurements: by using laser Doppler Iontophoresis and by using the Glycocheck (www. 
glycocheck.com). Local microvascular skin blood flow of the palmar side of the forearm was measured using single-point laser-Doppler flowmetry (Periflux 5000 system; Perimed A.B., Sweden) before and after pharmacologically induced vasodilatation. Blood perfusion was recorded in perfusion units (PU), which correlate with the number and velocity of passing blood cells in the microvasculature of the skin. Endothelium-dependent vasodilatation was induced by acetylcholine, which is known to release nitric oxide and prostanoids from the endothelium (Turner et al., 2008). Endothelium-independent vasodilatation was induced by nitroprusside, a direct nitric oxide donor (Feelisch, 1998). Administration of both drugs across the skin was realized by iontophoresis, a technique that uses a weak current to transport substances in ionic form across the skin. The drug delivery electrode was incorporated in the laser-Doppler probe. The study protocol prescribed multiple successive doses resulting in a cumulative dose response curve. For acetylcholine ( $1 \%$ solution, Miochol, Novartis Pharma) seven successive doses were administered with a 60-s interval, and for nitroprusside ( $25 \mathrm{mg} / \mathrm{ml}$ nitroprusside-sodium-dihydrate $0.1 \%$ solution, sodium nitroprusside) nine doses with a 90-s interval were used. Administration required 20 s of iontophoresis using a $0.10 \mathrm{~mA}$ anodal current (acetylcholine) or a $0.20 \mathrm{~mA}$ cathodal current (nitroprusside). For statistical analysis, the average perfusion values in a steady period of at least $30 \mathrm{~s}$ without significant movement artefacts were taken at baseline (before administration of the drugs) and at maximum attained perfusion after drug delivery, by use of specially designed software (Perisoft, Perimed A.B., Sweden) (Touwslager et al., 2012). All analyses of the laser-Doppler signal were conducted by one experienced researcher (H.Z.). The maximum value was corrected for the baseline value using regression analysis.

\section{Cortisol/cortisone levels}

As a marker of chronic stress, cortisol and cortisone levels in hair were analysed. It has been suggested that altered activity of the hypothalamus-pituitary-adrenal (HPA) axis leading to the secretion of glucocorticoid hormones may play an important role in the development of metabolic syndrome (Stalder et al., 2013). Hair strands ( $3 \mathrm{~mm}$ in diameter) were taken from the posterior vertex region, as close to the scalp as possible. Cortisol concentrations were examined using chromatography, from the $3 \mathrm{~cm}$ hair segment closest to the scalp. These parts are assumed to represent cumulative cortisol exposure (and thus HPA activity) over the past three months (Meyer and Novak, 2012, Sauvé et al., 2007). Hair specific characteristics were noted (i.e. washes per week and hair length). A hair sample was taken from all children whose parents consented and who had hair longer than $3 \mathrm{~cm}$.

\section{Statistical analysis}

All analyses were conducted in SPSS version 24.0 (IBM-SPSS, Chicago, IL, USA). Histograms, normal Q-Q plots, and box plots were used to visualize distribution of the data. Because all data were within 4SDs from the mean, the linear regression was considered suitable 
for between-group comparison of continuous variables (Skovlund and Fenstad, 2001). Differences in categorical outcomes were analyzed using logistic regression. Possible confounders (mode of conception (IVF/ICSI), gender of the child, amount of physical exercise of the child and the parents, weight, (duration of) breastfeeding, height and BMI of the parents, level of education of the parents, smoking behavior of the parents, parity of the mother, family history of cardiac disease, diabetes or hypertension) were controlled for by use of multivariable linear regression. For each outcome measure, only confounders that altered the beta for culture medium $>10 \%$ in a bivariable analysis were retained and introduced in the multivariable model. In all cases, P-values represent two-sided P-values after correction for confounders using multivariable regression analysis. To compare results of blood samples with the control group, a linear regression analysis was used, using dummy variables to allow for comparison between the three groups. Testing for homogeneity of variances was performed using Levene's test of equality. Differences in baseline characteristics between the participants and non-participants were analyzed using a logistic regression analysis. P-values of $<0.05$ were considered to reflect statistical significance. 


\section{RESULTS}

A total of 294 singletons were eligible to participate in the study, 126 children born after embryo culture in Cook medium and 168 after culture in Vitrolife medium. Out of the 294 parent couples, 9 were lost to follow-up because of living abroad or failure to trace them. Of the remaining 285, a total of 136 (47.7\%) parent couples agreed for their child to participate and signed written informed consent; 61 (50.8\%) in the Cook group, and 75 (45.5\%) in the Vitrolife group. The baseline characteristics at the time of birth of the participating children were comparable to those of the children not participating in the study (Table I, Chapter 3). Neonatal outcome and baseline characteristics of the participating children were compared between the culture medium groups, showing a difference in birthweight in keeping with the full cohort (Table I, Chapter 3). We also checked for the presence of major or minor congenital abnormalities. A major congenital abnormality was reported in only 2 children ( 1 mild hip dysplasia and 1 unilateral duplicated ureter). Baseline characteristics of the parents were compared for the two groups (Table II, Chapter 3). Some small differences were seen in level of education, smoking behavior, weight and height of the parents. If necessary according to the bivariable analysis, these possible confounders were included in the multivariable analysis.

Age at the day of measurement was 9.5 years (range 9.1 to 10.3 years) for the Cook children and 9.5 years (range 9.1 to 10.5) for the Vitrolife children.

\section{Blood pressure}

Differences in systolic (adj. beta 0.364 [95\% Cl -2.129 to 2.856]) and diastolic (adj. beta 0.275 [95\% Cl -2.105 to 2.654$]$ ) blood pressures ( $\mathrm{mmHg}$ ) between the two groups were small and not statistically significant. Also resting heart rate was comparable (Table I). In both groups SD-scores of blood pressure were close to 0 , indicating that the average blood pressures are comparable to the average blood pressures of the reference population. No significant differences were observed for SD-scores between the two groups. The multivariable regression analysis did not show significant association between culture medium and blood pressure values.

\section{Blood samples}

After an overnight fast, cholesterol, glucose, insulin, LDL, HDL and triglyceride levels were within the normal ranges for both groups. There were no statistically significant differences between the two groups. TSH ( $\mathrm{mU} / \mathrm{L})$ did not differ between the groups either. In both groups, only 3 children had a TSH higher than $5 \mathrm{mU} / \mathrm{L}$, all with normal T4 values. This is considered to reflect a subclinical hypothyroidism. The results are shown in table II. Results of both culture medium groups were also compared with a control group of 
healthy non-IVF children. There was a significant difference in triglyceride levels and HDL levels between both culture media groups and the control group. HDL was significantly lower in the control group and the triglycerides were significantly higher in the control group compared to the IVF/ICSI groups. Also LDL was lower in the Cook group compared to the control group. Results are shown in table III.

\section{Endothelial function}

Endothelial function in the microcirculation was analyzed by iontophoresis with maximum perfusion units corrected for the baseline value as a measure for vasodilation capacity; results are shown in table IV. There were no differences in baseline values between the two groups and between the consecutive measurements (Acetylcholine and Nitroprusside). After correction for confounders in a multivariable regression model, the results remained the same.

\section{Cortisol and cortisone concentration in hair}

Both cortisol and cortisone concentrations in the hair samples were comparable for the two groups (table V). After multivariable regression with correction for possible confounders no significant relation was found between culture medium and cortisol or cortisone levels (Cortisol: adj. beta -1.521 [95\%Cl - 4.66 to 1.63]); Cortisone: adj. beta 1.698 [95\%Cl -0.92 to 4.31$])$.

Table I - Blood pressure and heart rate

\begin{tabular}{|c|c|c|c|c|}
\hline & Cook $(n=61)^{(a)}$ & Vitrolife $(\mathrm{N}=75)^{(a)}$ & Adj Beta (bc) & P-value \\
\hline Systolic blood pressure [mmHg] ${ }^{(\mathrm{ci})}$ & $99.8(7.6)$ & $101.0(7.3)$ & $0.364[-2.129$ to 2.856$]$ & 0.773 \\
\hline Z-Score Systolic blood pressure (ci) & $-0.22(0.71)$ & $-0.13(0.71)$ & $0.034[-0.205$ to 0.273$]$ & 0.780 \\
\hline Diastolic blood pressure [mmHg] (cii) & $60.1(7.2)$ & $60.3(6.5)$ & $0.275[-2.105$ to 2.654$]$ & 0.820 \\
\hline Z-Score Diastolic Blood Pressure (cii) & $-0.08(0.65)$ & $-0.06(0.61)$ & $0.005[-0.213$ to 0.223$]$ & 0.965 \\
\hline Heart rate [bpm] ${ }^{\text {(ciii) }}$ & $77.0(8.9)$ & $79.0(9.3)$ & $2.473[-0.747$ to 5.694$]$ & 0.131 \\
\hline
\end{tabular}

a) Data presented as mean (SD)

b) Adjusted beta reflects the effect size and direction of the association of culture medium with the parameter [95\%Cl]

c) Adjusted when necessary (>10\% effect on adjusted Beta for culture medium) for relevant confounders using a multivariable regression model

i. Gender of the child, height of the child, smoking behavior of father and mother, family history of hypertension or cardiac disease of father and mother, level of education of father and mother.

ii. Gender of the child, smoking behavior of father and mother, level of education of father and mother

iii. Gender of the child, smoking behaviour of father and mother, level of education of mother, family history of hypertension of cardiac disease of mother 
Table II - Blood values of IVF children and control group

\begin{tabular}{|c|c|c|c|c|}
\hline & Cook $(n=54)^{(a)}$ & Vitrolife $(n=53)^{(a)}$ & Adj. Beta culture medium (bc) & P-value (bc) \\
\hline $\mathrm{HbA} 1 \mathrm{c}[\mathrm{mmol} / \mathrm{mol} \mathrm{Hb}]^{(\mathrm{ci})}$ & $32.34(2.95)$ & $32.19(2.76)$ & $-0.238[-1.428$ to 0.951$]$ & 0.692 \\
\hline Fasting glucose [mmol/L] (ci) & $4.74(0.36)$ & $4.70(0.33)$ & $-0.048[-0.181$ to 0.085$]$ & 0.472 \\
\hline Fasting insulin [pmol/L] ${ }^{(\mathrm{ci})}$ & $39.15(34.94)$ & $36.79(33.71)$ & -6.340 [-20.278 to 7.598$]$ & 0.369 \\
\hline Total cholesterol [mmol/L] (cii) & $4.27(0.63)$ & $4.33(0.76)$ & $0.139[-0.141$ to 0.418$]$ & 0.328 \\
\hline HDL cholesterol [mmol/L] (cii) & $1.67(0.34)$ & $1.65(0.43)$ & $0.036[-0.118$ to 0.190$]$ & 0.644 \\
\hline LDL cholesterol [mmol/L] (cii) & $2.26(0.48)$ & $2.35(0.59)$ & $0.117[-0.100$ to 0.335$]$ & 0.286 \\
\hline Triglycerides [mmol/L] (cii) & $0.82(0.65)$ & $0.78(0.39)$ & $-0.035[-0.255$ to 0.184$]$ & 0.751 \\
\hline TSH [mU/L] ${ }^{(\mathrm{ciii})}$ & $2.75(1.17)$ & $3.06(1.77)$ & $0.220[-0.371$ to 0.811$]$ & 0.462 \\
\hline
\end{tabular}

HbA1c=haemog/obin A1c; H/LDL = high / low density lipoprotein; TSH = thyroid stimulating hormone.

a. Data presented as mean (SD)

b. Adjusted beta reflects the effect size and direction of the association of culture medium with the parameter [95\% Cl]

c. Adjusted when necessary (>10\% effect on adjusted Beta for culture medium) for relevant confounders using a multivariable regression model

i. Gender, weight, BMI, waist/hip ratio, level of education of the parents, smoking behaviour of the father

ii. Gender, weight, waist/hip ratio

iii. Gender, weight, waist/hip ratio, level of education of parents, smoking behaviour of the father

Table III - Results of IVF children compared to control group

\begin{tabular}{|c|c|c|c|c|c|}
\hline & $\begin{array}{l}\text { Control } \\
(n=60)^{(a)}\end{array}$ & $\begin{array}{l}\text { Beta } \\
\text { Cook vs. Control (b) }\end{array}$ & P-value & $\begin{array}{l}\text { Beta } \\
\text { Vitrolife vs. Control (b) }\end{array}$ & P-value \\
\hline Total cholesterol [mmol/L] & $4.29(0.66)$ & -0.019 & 0.881 & 0.044 & 0.731 \\
\hline HDL cholesterol [mmol/L] & $1.33(0.30)$ & 0.346 & $<0.001$ & 0.323 & $<0.001$ * \\
\hline LDL cholesterol [mmol/L] & $2.49(0.57)$ & -0.217 & 0.036 & -0.133 & 0.200 \\
\hline $\begin{array}{l}\text { Triglycerides } \\
{[\mathrm{mmol} / \mathrm{L}]}\end{array}$ & $1.06(0.52)$ & -0.240 & 0.017 & -0.274 & $0.007 *$ \\
\hline $\mathrm{TSH}[\mathrm{mU} / \mathrm{L}]$ & $3.23(1.67)$ & -0.476 & 0.106 & -0.166 & 0.575 \\
\hline
\end{tabular}


Table IV - Endothelial function assessed by iontophoresis with laser- doppler

\begin{tabular}{|c|c|c|c|c|}
\hline & Cook $(n=57)^{(a)}$ & Vitrolife $(n=70)^{(a)}$ & Adj. Beta culture medium (de) & P-value (de) \\
\hline Acetylcholine baseline (bei) & $4.3(7.4)$ & $7.0(17.2)$ & $3.480[-2.084$ to 9.043$]$ & 0.218 \\
\hline $\begin{array}{l}\text { Acetylcholine max perfusion } \\
\text { (cei) }\end{array}$ & $91.4(50.4)$ & $93.9(48.9)$ & $7.404[-12.453$ to 27.261$]$ & 0.461 \\
\hline Nitroprusside baseline (beii) & $6.2(10.3)$ & $8.3(24.5)$ & $1.926[-5.296$ to 9.148$]$ & 0.598 \\
\hline $\begin{array}{l}\text { Nitroprusside max perfusion } \\
\text { (ceii) }\end{array}$ & $115.1(59.1)$ & $91.8(60.7)$ & $-7.366[-28.033$ to 13.301$]$ & 0.481 \\
\hline
\end{tabular}

a. Data presented as mean (SD)

b. Lowest value at the start of measurement, no medication administered

c. Highest value after maximum administration of drug

d. Adjusted beta reflects the effect size and direction of the association of culture medium with the parameter [95\% Cl]

e. Adjusted when necessary (>10\% effect on adjusted Beta for culture medium) for relevant confounders using a multivariable regression model

i. Age, gender, height, smoking behaviour of father and mother, family history of hypertension or cardiac disease, birthweight, growth velocity in the first month of life, level of education of the parents

ii. Age, gender, smoking behaviour of father and mother, family history of hypertension or cardiac disease, birthweight, growth velocity in the first month of life.

Table V - Cortisol and cortisone concentration in hair samples

\begin{tabular}{lllll}
\hline & Cook $(n=49){ }^{(a)}$ & Vitrolife $(n=58)^{(a)}$ & Adj.Beta culture medium ${ }^{(b c)}$ & P-value ${ }^{(b c)}$ \\
\hline $\begin{array}{l}\text { Cortisol Concentration [pg/ } \\
\text { mg hair] }\end{array}$ & $4.99(11.0)$ & $3.80(5.5)$ & $-1.521[-4.657$ to 1.632] & 0.341 \\
$\begin{array}{l}\text { Cortison Concentration [pg/ } \\
\text { mg hair] (ci) }\end{array}$ & $12.92(7.32)$ & $13.33(7.6)$ & $1.698[-0.917$ to 4.313] & 0.201 \\
\hline
\end{tabular}

a. Data presented as mean (SD)

b. Adjusted beta reflects the effect size and direction of the association of culture medium with the parameter [95\% Cl]

c. Adjusted when necessary (>10\% effect on adjusted Beta for culture medium) for relevant confounders using a multivariable regression model

i. Frequency of washing hairs, length of the hairs, gender, age, BMI 


\section{DISCUSSION}

This study evaluates the cardiovascular effects of IVF culture medium on IVF children at the age of nine years. We did not find significant differences in cardiovascular parameters between children born after IVF culture in 2 different commercially available culture media.

Blood pressure values were compared with an American reference (non-IVF) cohort by calculating percentiles and SD-scores (National, 2004). The average SD-scores in both culture medium groups were around the average of the reference population (zero) and no significant differences between the culture medium groups were observed. Our findings are in agreement with a recent study by Kuiper et al. who found that cardiovascular development (blood pressure and anthropometrics) for conventional IVF with hyperstimulation compared to IVF in the modified natural cycle and spontaneously conceived children at 9 years of age was comparable (Kuiper et al., 2017). Several other studies found differences in blood pressure in children born after IVF if compared with children born after natural conception (Belva et al., 2007, Ceelen et al., 2008, Guo et al., 2017, Sakka et al., 2010). The fact that we do not see a difference in blood pressure between our IVF groups and the reference population might indicate the inferior quality of a general reference population in comparison to a properly matched control group or a control group using different IVF protocols. Nevertheless, our results indicate that the blood pressure differences seen at early ages in other studies are not necessarily caused by the culture medium.

In our study we did not find differences in any of the blood sample measurements between the two culture medium groups at 9 years of age. Also, all values were within the normal ranges, which is reassuring for now. In a study by Pontesilli and co-workers (2015) significantly higher (although all within the normal ranges) fasting glucose levels at 5-6 years of age were found if IVF/ICSI and ovulation induction cycles were compared to natural conception cycles from fertile parents. They found an average value of $4.7 \mathrm{mmol} / \mathrm{L}$ for fasting glucose in the IVF/ICSI children, which was the same as in our study. No difference was observed if children born after natural conception (NC) from fertile parents were compared to children born after NC from subfertile parents (Pontesilli et al., 2015), suggesting that treatment factors associated with ART rather than patient factors are responsible for the observed differences. However, we did find significant differences in HDL, LDL and triglyceride levels between the control group of healthy non-IVF children and the two culture medium groups, with HDL levels being lower, LDL levels higher and triglyceride levels being higher in the control group. This might suggest a slightly favorable lipid profile in the IVF/ ICSI group, however, this was not confirmed by the other lipid measurements and total cholesterol level. Comparable results were presented in a study showing that children born after IVF with fresh embryo transfer had more favorable lipid profiles than the frozen 
embryo transfer group and the naturally conceived controls (Green et al., 2013). Also, in our study all levels were within the normal ranges. Also, this was an uncorrected analysis since information on confounding factors was not available for our control group. Most likely the differences in HDL and triglycerides concern a chance event that does not hold clinical relevance, however further research is necessary on this topic. TSH levels were normal for almost all IVF/ICSI children and were comparable to the non-IVF control group. This was in contrast with findings from several other studies where elevated TSH levels were observed in IVF children if compared to NC children (Lv et al., 2014, Onal et al., 2012).

As expected based on several other studies, the results of endothelial function analysis using iontophoresis showed a wide variance in the observed values. Despite the wide variance in observations, a high reproducibility is reported, resulting in valuable information on microcirculation reactivity (Cracowski et al., 2006). This makes the method a reliable and non-invasive way of assessing endothelial function. The reproducibility of our baseline data between arms was good. Both baseline and maximum values were comparable to data from other studies. In our study group there were no significant differences between the two groups. Earlier, it was reported that birthweight and also growth in the first month of life was inversily correlated with endothelial function (Singhal and Lucas, 2004, Touwslager et al., 2012). However, in our study groups, which differed in birth weight, no significant differences were seen at the age of 9 years. In a study by Scherrer and co-workers (2012) cardiovascular function as well as endothelial function was assessed at high altitude. They compared children born after IVF/ICSI with matched controls born after natural conception and with children born after ovulation induction with hormonal stimulation. They observed a significantly reduced cardiovascular function in IVF/ICSI children, including reduced endothelial function as assessed by flow mediated dilation (FMD) compared to both control groups (Scherrer et al., 2012).

The strength of our study is the alternating allocation of culture media. Although not as perfect as randomization, this is the first and therewith oldest cohort of children where culture medium was allocated alternatingly and used in a blinded setting, in the same period, with all other culture conditions identical. Also, the current study was performed in a standardized way by two experienced investigators who were blinded - as were the children and their parents - for the culture medium group the child belonged to. However, this study also has limitations. One of the limitations is the relatively low participation rate of $47.7 \%$, although characteristics of participants and non-participants were similar. Since we had no permission to contact the non-participants, it was not possible to determine whether all baseline characteristics were still the same at the time of measurement. With the current number of participants we were able to rule out differences as little as $3 \mathrm{mmHg}$ in systolic and diastolic blood pressures. It is questionable whether a smaller difference 
would have any clinical relevance. It is questionable whether a smaller difference would have any clinical relevance. Also, our study lacked a control group with naturally conceived children for blood pressure, endothelial function and glucose levels, making it impossible to draw conclusions on how the observed values relate to a comparable group of Western European naturally conceived children.

The observations in our study give no cause for concern regarding cardiovascular development of the children in our cohort at this moment. However, as weight and body composition differ between the groups (Chapter 3), it is important that long-term health will be closely monitored. Furthermore, the observations in animal studies and the available research on the DOHaD hypothesis still warrant close, structured long-term cardiovascular follow up of IVF children. Possibly, adverse cardiovascular effects might emerge later in adolescence or adulthood. 


\section{REFERENCES}

Barbuscia A, Mills MC. Cognitive development in children up to age 11 years born after ART-a longitudinal cohort study. Hum Reprod 2017: 1-7.

Barker DJ. Fetal origins of cardiovascular disease. Ann Med 1999;31 Suppl 1: 3-6.

Barker DJ. Adult consequences of fetal growth restriction. Clin Obstet Gyneco/ 2006;49: 270-283.

Belva F, Henriet S, Liebaers I, Van Steirteghem A, Celestin-Westreich S, Bonduelle M. Medical outcome of 8-year-old singleton ICSI children (born >or=32 weeks' gestation) and a spontaneously conceived comparison group. Hum Reprod 2007;22: 506-515.

Bergh C, Wennerholm UB. Obstetric outcome and long-term follow up of children conceived through assisted reproduction. Best Pract Res Clin Obstet Gynaecol 2012;26: 841-852.

Calle A, Fernandez-Gonzalez R, Ramos-Ibeas P, Laguna-Barraza R, Perez-Cerezales S, Bermejo-Alvarez P, Ramirez MA, Gutierrez-Adan A. Long-term and transgenerational effects of in vitro culture on mouse embryos. Theriogenology 2012;77: 785-793.

Ceelen M, van Weissenbruch MM, Prein J, Smit J, Vermeiden JP, Spreeuwenberg M, van Leeuwen FE, Delemarre-van de Waal HA. Growth during infancy and early childhood in relation to blood pressure and body fat measures at age 8-18 years of IVF children and spontaneously conceived controls born to subfertile parents. Hum Reprod 2009;24: 2788-2795.

Ceelen M, van Weissenbruch MM, Roos JC, Vermeiden JP, van Leeuwen FE, Delemarre-van de Waal HA. Body composition in children and adolescents born after in vitro fertilization or spontaneous conception. J Clin Endocrinol Metab 2007;92: 3417-3423.

Ceelen M, van Weissenbruch MM, Vermeiden JP, van Leeuwen FE, Delemarre-van de Waal HA. Cardiometabolic differences in children born after in vitro fertilization: follow-up study. J Clin Endocrinol Metab 2008;93: 1682-1688.

Chen M, Wu L, Zhao J, Wu F, Davies MJ, Wittert GA, Norman RJ, Robker RL, Heilbronn LK. Altered glucose metabolism in mouse and humans conceived by IVF. Diabetes 2014;63: 3189-3198.

Cracowski JL, Minson CT, Salvat-Melis M, Halliwill JR. Methodological issues in the assessment of skin microvascular endothelial function in humans. Trends Pharmacol Sci 2006;27: 503-508.

Dumoulin JC, Land JA, Van Montfoort AP, Nelissen EC, Coonen E, Derhaag JG, Schreurs IL, Dunselman GA, Kester $A D$, Geraedts JP et al. Effect of in vitro culture of human embryos on birthweight of newborns. Hum Reprod 2010;25: 605-612.

Feelisch M. The use of nitric oxide donors in pharmacological studies. Naunyn Schmiedebergs Arch Pharmacol 1998;358: 113-122.

Fernández-Gonzalez R, Moreira P, Bilbao A, Jiménez A, Pérez-Crespo M, Ramírez MA, Rodríguez De Fonseca F, Pintado B, Gutiérrez-Adán A. Long-term effect of in vitro culture of mouse embryos with serum on mRNA expression of imprinting genes, development, and behavior. Proc Natl Acad Sci U S A 2004;101: 5880-5885.

Feuer S, Rinaudo P. Preimplantation stress and development. Birth Defects Res C Embryo Today 2012;96: 299-314. Feuer SK, Liu X, Donjacour A, Lin W, Simbulan RK, Giritharan G, Piane LD, Kolahi K, Ameri K, Maltepe E et al. Use of a mouse in vitro fertilization model to understand the developmental origins of health and disease hypothesis. Endocrinology 2014;155: 1956-1969.

Gluckman PD, Hanson MA, Bateson P, Beedle AS, Law CM, Bhutta ZA, Anokhin KV, Bougneres P, Chandak GR, Dasgupta $\mathrm{P}$ et al. Towards a new developmental synthesis: adaptive developmental plasticity and human disease. Lancet 2009;373: 1654-1657.

Green MP, Mouat F, Miles HL, Hopkins SA, Derraik JG, Hofman PL, Peek JC, Cutfield WS. Phenotypic differences in children conceived from fresh and thawed embryos in in vitro fertilization compared with naturally conceived children. Fertil Steril 2013;99: 1898-1904. 
Guo XY, Liu XM, Jin L, Wang TT, Ullah K, Sheng JZ, Huang HF. Cardiovascular and metabolic profiles of offspring conceived by assisted reproductive technologies: a systematic review and meta-analysis. Fertil Steril 2017;107: 622-631 e625.

Hart R, Norman RJ. The longer-term health outcomes for children born as a result of IVF treatment: Part I--General health outcomes. Hum Reprod Update 2013;19: 232-243.

Jackson RA, Gibson KA, Wu YW, Croughan MS. Perinatal outcomes in singletons following in vitro fertilization: a meta-analysis. Obstet Gynecol 2004;103: 551-563.

Kleijkers SH, van Montfoort AP, Smits LJ, Viechtbauer W, Roseboom TJ, Nelissen EC, Coonen E, Derhaag JG, Bastings $\mathrm{L}$, Schreurs IE et al. IVF culture medium affects post-natal weight in humans during the first 2 years of life. Hum Reprod 2014;29: 661-669.

Kuiper D, Hoek A, la Bastide-van Gemert S, Seggers J, Mulder DJ, Haadsma M, Heineman MJ, Hadders-Algra M. Cardiovascular health of 9-year-old IVF offspring: no association with ovarian hyperstimulation and the in vitro procedure. Hum Reprod 2017;32: 2540-2548.

Lv PP, Meng Y, LV M, Feng C, Liu Y, Li JY, Yu DQ, Shen Y, Hu XL, Gao Q et al. Altered thyroid hormone profile in offspring after exposure to high estradiol environment during the first trimester of pregnancy: a cross-sectional study. BMC Med 2014;12: 240.

Market-Velker BA, Fernandes AD, Mann MR. Side-by-side comparison of five commercial media systems in a mouse model: suboptimal in vitro culture interferes with imprint maintenance. Biol Reprod 2010;83: 938-950.

Meyer JS, Novak MA. Minireview: Hair cortisol: a novel biomarker of hypothalamic-pituitary-adrenocortical activity. Endocrinology 2012;153: 4120-4127.

National. High Blood Pressure Education Program Working Group on High Blood Pressure in Children and Adolescents. The fourth report on the diagnosis, evaluation, and treatment of high blood pressure in children and adolescents. Pediatrics 2004;114: 555-576.

Nelissen EC, Van Montfoort AP, Smits LJ, Menheere PP, Evers JL, Coonen E, Derhaag JG, Peeters LL, Coumans AB, Dumoulin JC. IVF culture medium affects human intrauterine growth as early as the second trimester of pregnancy. Hum Reprod 2013;28: 2067-2074.

Onal H, Ercan O, Adal E, Ersen A, Onal Z. Subclinical hypothyroidism in in vitro fertilization babies. Acta Paediatr 2012;101: e248-252.

Pinborg A, Wennerholm UB, Romundstad LB, Loft A, Aittomaki K, Söderström-Anttila V, Nygren KG, Hazekamp J, Bergh C. Why do singletons conceived after assisted reproduction technology have adverse perinatal outcome? Systematic review and meta-analysis. Hum Reprod Update 2013;19: 87-104.

Pontesilli M, Painter RC, Grooten IJ, van der Post JA, Mol BW, Vrijkotte TG, Repping S, Roseboom TJ. Subfertility and assisted reproduction techniques are associated with poorer cardiometabolic profiles in childhood. Reprod Biomed Online 2015;30: 258-267.

Sakka SD, Loutradis D, Kanaka-Gantenbein C, Margeli A, Papastamataki M, Papassotiriou I, Chrousos GP. Absence of insulin resistance and low-grade inflammation despite early metabolic syndrome manifestations in children born after in vitro fertilization. Fertil Steril 2010;94: 1693-1699.

Sauvé B, Koren G, Walsh G, Tokmakejian S, Van Uum SH. Measurement of cortisol in human hair as a biomarker of systemic exposure. Clin Invest Med 2007;30: E183-191.

Scherrer U, Rimoldi SF, Rexhaj E, Stuber T, Duplain H, Garcin S, de Marchi SF, Nicod P, Germond M, Allemann Y et al. Systemic and pulmonary vascular dysfunction in children conceived by assisted reproductive technologies. Circulation 2012;125: 1890-1896

Scott KA, Yamazaki Y, Yamamoto M, Lin Y, Melhorn SJ, Krause EG, Woods SC, Yanagimachi R, Sakai RR, Tamashiro $\mathrm{KL}$. Glucose parameters are altered in mouse offspring produced by assisted reproductive technologies and somatic cell nuclear transfer. Biol Reprod 2010;83: 220-227. 
Singhal A, Lucas A. Early origins of cardiovascular disease: is there a unifying hypothesis? Lancet 2004;363: 16421645.

Skovlund E, Fenstad GU. Should we always choose a nonparametric test when comparing two apparently nonnormal distributions? J Clin Epidemiol 2001;54: 86-92.

Stalder T, Kirschbaum C, Alexander N, Bornstein SR, Gao W, Miller R, Stark S, Bosch JA, Fischer JE. Cortisol in hair and the metabolic syndrome. J Clin Endocrinol Metab 2013;98: 2573-2580.

Touwslager RN, Gerver WJ, Tan FE, Gielen M, Zeegers MP, Zimmermann LJ, Houben AJ, Blanco CE, Stehouwer CD, Mulder AL. Influence of growth during infancy on endothelium-dependent vasodilatation at the age of 6 months. Hypertension 2012;60: 1294-1300.

Touwslager RN, Houben AJ, Gielen M, Zeegers MP, Stehouwer CD, Zimmermann LJ, Kessels AG, Gerver WJ, Blanco CE, Mulder AL. Endothelial vasodilatation in newborns is related to body size and maternal hypertension. J Hypertens 2012;30: 124-131.

Turner J, Belch J, Khan F. Current concepts in assessment of microvascular endothelial function using laser Doppler imaging and iontophoresis. Trends Cardiovasc Med 2008;18: 109-116.

Watkins AJ, Platt D, Papenbrock T, Wilkins A, Eckert J, Kwong WY, Osmond C, Hanson M, Fleming TP. Mouse embryo culture induces changes in postnatal phenotype including raised systolic blood pressure. Proc Natl Acad Sci USA 2007;104: 5449-5454.

Zandstra H, Van Montfoort AP, Dumoulin JC. Does the type of culture medium used influence birthweight of children born after IVF? Hum Reprod 2015;30: 2693. 


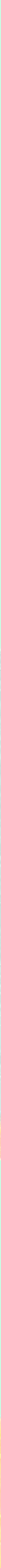




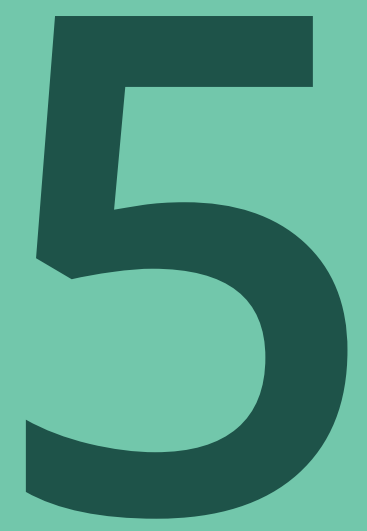

\section{No effect of IVF culture medium on cognitive development of 9-year-old children}

Zandstra H, Smits LJ, van Kuijk SMJ, van Golde RJT, Evers JLH, Dumoulin JCM, van Montfoort APA

Human Reproduction Open, 2018 Sep; Issue 4, pp 1-8 


\section{ABSTRACT}

\section{Study Question}

Do embryo culture media used during an IVF/ICSI treatment have an effect on cognitive development of 9-year-old singleton IVF children?

\section{Summary Answer}

Cognitive development of children born after culture in two different embryo culture media is comparable.

\section{What is known already?}

Previously, we have shown that the culture medium used in an IVF/ICSI treatment affects birthweight and weight at 2 years of age (Dumoulin et al., 2010, Kleijkers et al., 2014) after alternating assignment to embryo culture in either K-SCICM (Cook) or G1 ${ }^{\mathrm{TM}}$ Version 3 (Vitrolife). Children with low birthweight are known to have an increased risk for learning disabilities. Data on cognitive development in general of children born after ART is still conflicting, and the only study reporting on effects of culture medium on cognitive development shows significant differences in cognitive development between 2 culture medium groups (Bouillon et al., 2016).

\section{Study Design, Size and Duration}

In this observational cohort follow-up study (MEDIUM-KIDS), parents of all singletons from our abovementioned study were approached after the ninth birthday of their child to participate in an additional follow-up study. Of the 294 eligible children included in the original study, 119 children (70 Vitrolife and 49 Cook) participated in the current study.

\section{Participants/Materials, Settings and Methods}

All follow-up measurements were performed between March 2014 and December 2016. CITO (Dutch Central Institute for Test Development) developed the Dutch pupil monitoring system, which involves nationwide independent, standardized, academic achievement score-tests to monitor the child's school performance twice a year at fixed time points from $3^{\text {rd }}$ grade onwards. The tests include language skills (vocabulary and orthography), math and reading capability and comprehension. Results from the tests performed between $3^{\text {rd }}$ and $6^{\text {th }}$ grade, expressed as ability scores, were obtained from the school the child went to, after written informed consent from both parents. To investigate school performance development over the years, we used a mixed effects multilevel model. The least complex model with the best fit was selected to analyse whether culture medium affects cognitive development in our cohort. 


\section{Main results and the role of chance}

For all domains, the random intercept model was used. All analyses showed comparable results for the two culture medium groups. No significant differences were observed for any of the cognitive development domains, even after correction for potential confounders. Parental level of education was higher in the IVF group (45\%) if compared to the national average level of education (35\%), which most likely explains the higher CITO scores for the IVF children if compared to the National ability scores.

\section{Limitations, reasons for caution}

A limitation of the study was its pseudo-randomised design and the relatively low participation rate of $40.5 \%$. This and the number of missing data prevent us from drawing robust causal conclusions from the observed association. However, as this is the first and therewith oldest cohort of children where culture medium was allocated alternatingly and used in a blinded setting, in the same period, with all other conditions identical this study gives up until now the best available evidence. No differences were seen in baseline characteristics between participants and non-participants, the study had enough power to detect a difference in ability score that reflects a shift of at least one performance category between the 2 groups.

\section{Wider implications of the findings}

Our study analyzes effects of culture medium on school performance of children born after IVF/ICSI in a prospective cohort study. Although further research on long-term academic skills and also on behavior is essential, our results are reassuring and should make parents of children born after IVF/ICSI feel comfortable with their children's cognitive development. 


\section{INTRODUCTION}

Children born after IVF are known to have an increased risk of adverse perinatal outcomes, such as low birthweight and preterm birth, compared to children born after natural conception (Bergh and Wennerholm, 2012, Jackson et al., 2004, Pinborg et al., 2013). Studies on long-term outcome of the children born after IVF are scarce. There is growing evidence that culture medium can affect birthweight of children born after ART, however the comparison of studies analysing these effects is complicated by the fact that each study compares different culture media with different compositions (Zandstra et al., 2015).

It is known that adverse perinatal outcomes such as preterm birth and low birthweight are associated with a higher incidence of learning disabilities and attention deficit hyper activity disorder (Murray et al., 2016). Also, it is known that intelligence quotient (IQ) is related to birthweight and that the childhood IQ increases within the normal birthweight ranges (Matte et al., 2001). It is also known that children born preterm are at increased risk of poor academic outcome (Twilhaar et al., 2017).

Only a limited number of studies have been published on cognitive outcome of children born after IVF, with conflicting results. In 2013, a study published by Berry et al. (2013) found similar cognitive functions at 3 years of age if late preterm (LPT) children born after IVF were compared with LPT non-IVF children, suggesting that IVF itself at least not worsens the diminished cognitive development in late preterm born children (Berry et al., 2013). However, in children with normal perinatal outcome, a systematic review showed a possible negative effect on cognitive development caused by fertility treatments (Rumbold et al., 2017). Most high quality studies did not find differences if IVF children were compared with naturally conceived children, but some studies showed lower intelligence quotients (IQ) specifically among ICSI children (Rumbold et al., 2017). A recent study by Schendelaar et al. (2016) analyzed the effects of hormonal stimulation on cognitive outcome of the offspring. They found no causal relation between ovarian stimulation or the in-vitro procedure and the outcome. However, direct negative causal effects were found between severity of subfertility (defined by time to pregnancy) and cognition and behavior (Schendelaar et al., 2016). Only one study reporting on effects of culture medium on cognitive development has been published (Bouillon et al., 2016). They compared development of the offspring after a randomized study where they allocated IVF treatments to the use of one of two different culture media (SSM and Global). Neonatal and early childhood (up to 5 years) follow-up showed that the children in the Global group were significantly less likely than the children in the SSM group to show developmental problems (Bouillon et al., 2016). 
In 2010, we published the results of our study where a corrected difference of 112 grams in birthweight was observed in children born after IVF, if two different culture media were used alternatingly (Dumoulin et al., 2010). The children born after embryo culture in G1 version 3 (Vitrolife AB, Goteborg, Sweden) were heavier with an average birthweight of 3453 (SEM \pm 53 ) grams compared to the average birthweight of 3208 (SEM \pm 61 ) grams for the children born after embryo culture in K-SICM (Cook Medical, Brisbane, Australia)) $(P=0.003)$ (Dumoulin et al., 2010). Further follow-up showed that the weight difference between the two culture medium groups was still present at 9 years of age, likely to be caused by a higher adiposity (Zandstra et al., 2018). The difference in weight and BMI associated with culture medium was $1.58 \mathrm{~kg}$ [95\%Cl 0.01 to 3.14$] \mathrm{resp} .0 .84 \mathrm{~kg} / \mathrm{m}^{2}$ [95\%Cl 0.02 to 1.67 ] with Vitrolife children being heavier (Zandstra et al., 2018). Knowledge about effects of culture medium on cognitive development of children born after IVF is scarce and this is the oldest cohort with children born after embryo culture in two different culture media used alternatingly. Therefore, this population is extremely valuable for evaluating possible effects of culture medium on long-term cognitive development of children born after ART. Therefore, the aim of our study was to analyze the effect of culture medium on cognitive development of the children from this cohort 9 years after IVF. Furthermore we compared the IVF group with the average Dutch scores to gain insight in the cognitive performance of the IVF group compared to a non-IVF group 


\section{MATERIALS AND METHODS}

\section{Study design}

The current study is part of a prospective observational cohort study (MEDIUM-KIDS). The methods of the study are described in detail elsewhere (Zandstra et al., 2018). All children who participated in the MEDIUM-KIDS study, visited our hospital between March 2014 and December 2016. Parents and their child were invited to attend our clinic for a single visit, lasting approximately 2.5 hours. Information on possibly confounding factors, such as lifestyle, demographic data and medical history of the child and parents were recorded by means of a questionnaire, completed by the parents at the day of examination. As part of the follow up, school performance data were analysed.

\section{Ethical approval}

The study was registered in the Dutch Trial register (NTR4220) and the local ethics committee approved the study. Both parents of all children gave written informed consent.

\section{School performance}

To measure cognitive development of the children in our cohort, school performances were analyzed. In the Netherlands, primary school usually starts in September after the child turns 4 years old. The vast majority of schools (95\%) uses the test and monitor system developed by CITO (Centraal Instituut voor TestOntwikkeling, Dutch Central Institute for Test Development), i.e.the Dutch national pupil monitoring system, to give parents and teachers insight in the study progression of a child during primary school. Within this monitoring system regular testing is done. The first test starts in the third year of primary school at the age of 6 and tests are offered to all children twice a year on fixed time points. The test includes the following five domains: comprehensive reading, orthography, vocabulary, technical reading and mathematics. The test for comprehensive reading consists of a series of different texts with multiple-choice questions about the content of the text to test the child's understanding of the written text. Orthography is tested by a series of verbally presented words (read out loud by the teacher) that should be written down correctly by the child. Technical reading requires a Three-Minutes reading test which measures fluency and speed of word reading by counting the number of correctly read words from 3 cards containing separate words with an increasing level of difficulty. Vocabulary is tested by presenting separate words to the child with multiple-choice questions about the meaning of the word. Also, several mathematics skills including computational problems and problems on notion of time and the use of money are tested. 
The raw test scores on each domain are converted to a standardized individual ability score, which corrects for test content and difficulty and allows teachers to monitor progression in performance. It also allows a meaningful comparison between children, also in different classes and schools, which enables us to compare the two culture medium groups. Moreover, it allows a comparison with average results of all Dutch children. Average results for Dutch children were obtained from the CITO institution (CITO, 2016).

\section{Statistical analysis}

All analyses were conducted in SPSS version 23.0 (IBM-SPSS, Chicago, IL, USA). A Shapiro-Wilk test $(\mathrm{p}<0.05)$ and a visual inspection of the histograms, normal Q-Qplots, and box plots were used to test for normality of the data. For comparing baseline characteristics of the two groups, an unpaired Student t-test (for continuous variables) and logistic regression analysis were performed. To investigate school performance development over the years, we used a mixed effects multilevel model. This model enables us to compare the longitudinal school performance of all children while taking into account the dependency of data within one child. Furthermore it allows data to be unbalanced (i.e. participants do not have to be measured at the same timepoints). This analysis was conducted separately for each domain. The ability scores of all children were modelled against the number of months that the child received education at that particular test moment, expressed by "didactic age". The culture medium as well as several potentially confounding factors (parental education, gender of the child, age of the mother, smoking behaviour of the mother, ethnicity of the parents and the duration of being breastfed) were added to the model as a fixed factor and the children as a random factor to take into account the repeated measures. We used an unstructured variance-covariance matrix for the random effects and tested two covariance structures (unstructured and autoregressive 1) for the correlations of measures over time within children. The model fit was compared based on the restricted log likelihood (-2LL) and Akaike information criterion (AIC) using ChiSquare. Standardized ability scores were compared for all domains between both culture media groups and the Dutch reference population using a one sided T-test. 


\section{RESULTS}

The inclusion process is described in elsewhere (Zandstra et al., 2018). A total of 294 singletons were eligible to participate in the MEDIUM-KIDS study. Of the 136 children participating in the MEDIUM-KIDS study, we were able to retrieve the available CITO results of 119 children; 49 children in the Cook group and 70 in the Vitrolife group. The schools of the remaining 17 children did not use the CITO system or were using a different version of the system with results not comparable to the earlier versions. Not all children had results for all measuring moments since some measuring moments are optional and the schools can decide to skip those. Also, depending on the age at inclusion, not all children had already performed the mid $6^{\text {th }}$ grade test (M6). The baseline biomedical characteristics of the participating children were comparable to those of the children not participating in the study (table I). Neonatal outcome and baseline biomedical characteristics of the participating children were compared, showing a higher birthweight for the Vitrolife children, in keeping with the full cohort in the original study (Dumoulin et al., 2010). This was no longer significant due to the smaller subgroup sizes (table II). Baseline biomedical characteristics of the parents were compared for both groups (table III). Level of education of the fathers was significantly $(P=0.032)$ higher in the Vitrolife group. For the mothers, this was the opposite ( $P=0.089$ ). Next to other variables, having at least one higher educated parent or not was included in the models as a possible confounder.

For the linear mixed model we tested several models and covariance structures. The least complex model with the best fit was selected to analyse whether culture medium affects cognitive development in our cohort. For all domains, the unstructured matrix with random intercept model was chosen as the model with the best fit. All analysis showed comparable results for the two culture medium groups. No significant differences were observed for any of the cognitive development domains, even after correction for confounders; results are presented in figure 1.

The average ability scores of the IVF children were also compared cross-sectionally with the national average ability scores for all 5 domains. Both IVF groups performed significantly better compared to the average Dutch scores on almost all measuring moments on all 5 domains. The difference was significant on all measuring moments except for end of $3^{\text {rd }}$ grade for technical reading and mid $3^{\text {rd }}$ grade for vocabulary. The results for mid $5^{\text {th }}$ grade (=25 months didactic age) are presented in Figure 2 as an example. The presented differences were comparable for the other measuring moments (data not shown). 
Table I - Parental baseline characteristics participants versus non-participants ${ }^{(a)}$

\begin{tabular}{|c|c|c|c|}
\hline Characteristics & Participants $(n=119)^{(b c)}$ & Non-participants $(\mathrm{n}=175)^{(\mathrm{bc})}$ & P-value \\
\hline Duration of infertility (Yr) & $3.5(1.87)$ & $3.4(1.76)$ & 0.849 \\
\hline $\begin{array}{l}\text { Type of treatment } \\
\text { - ICSI } \\
\text { - IVF }\end{array}$ & $\begin{array}{l}79(66.4 \%) \\
40(33.6 \%)\end{array}$ & $\begin{array}{l}111(63.4 \%) \\
64(36.6 \%)\end{array}$ & 0.580 \\
\hline \multicolumn{4}{|c|}{ Maternal characteristics (at ovum pick up) } \\
\hline - Age (Yr) & $32.8(3.7)$ & $32.3(3.9)$ & 0.242 \\
\hline - Height (Cm) & $168.5(6.5)$ & $168.7(6.7)$ & 0.380 \\
\hline - Weight (Kg) & $69.3(10.0)$ & $68.5(10.7)$ & 0.551 \\
\hline - $\mathrm{BMI}(\mathrm{Kg} / \mathrm{m} 2)$ & $24.3(3.1)$ & $23.9(3.4)$ & 0.130 \\
\hline \multicolumn{4}{|c|}{ Paternal characteristics (at ovum pick up) } \\
\hline - Age (Yr) & $35.6(4.9)$ & $35.5(5.7)$ & 1.000 \\
\hline - Height (Cm) & $181.8(8.0)$ & $181.4(7.5)$ & 0.675 \\
\hline - Weight (Kg) & $86.3(11.8)$ & $83.8(13.5)$ & 0.122 \\
\hline - $\mathrm{BMI}(\mathrm{Kg} / \mathrm{m} 2)$ & $26.1(3.0)$ & $25.2(4.4)$ & 0.062 \\
\hline \multicolumn{4}{|l|}{ Neonatal characteristics } \\
\hline $\begin{array}{l}\text { Gestational age }(\mathbf{w k}) \\
\text { - Preterm birth }(<37 w k) \\
\text { - } \text { Very preterm birth }(<32 w k)\end{array}$ & $\begin{array}{l}39.6(1.8) \\
4(3.4 \%) \\
1(0.8 \%)\end{array}$ & $\begin{array}{l}39.2(3.5) \\
11(6.3 \%) \\
4(2.3 \%)\end{array}$ & $\begin{array}{l}0.280 \\
0.271 \\
0.367\end{array}$ \\
\hline $\begin{array}{l}\text { Birthweight (g) } \\
\text { - LBW }(<2500 \mathrm{gr}) \\
\text { - } \text { Very LBW }(<1500 \mathrm{gr})\end{array}$ & $\begin{array}{l}3403.2(531.5) \\
5(4.2 \%) \\
1(0.8 \%)\end{array}$ & $\begin{array}{l}3326.6(596.7) \\
11(6.3 \%) \\
4(2.3 \%)\end{array}$ & $\begin{array}{l}0.908 \\
0.442 \\
0.367\end{array}$ \\
\hline Gender (Boy) & 56 (47.1\%) & 85 (48.6\%) & 0.799 \\
\hline
\end{tabular}

a. Baseline characteristics in this table represent characteristics at the time of the IVF/ICSI treatment, since characteristics of non-responders could not be measured at the time of the study

b. Categorical data presented as $n(\%)=$ Number of cases (percentage of cases)

c. Continuous data presented as mean (SD) 
Table II - Baseline characteristics of participating children

\begin{tabular}{llll}
\hline Characteristics & Cook (n=49) (ab) & Vitrolife (n=70) (ab) & P-value \\
\hline Boys (n) & $24(49.0 \%)$ & $32(45.7 \%)$ & 0.725 \\
Method of conception & & & 0.604 \\
- ICSI & $31(63.3 \%)$ & $48(68.5 \%)$ & $22(31.4 \%)$ \\
- IVF & $18(36.7 \%)$ & & 0.693 \\
Embryotransfer & & $36(30.3 \%)$ & 0.979 \\
- SET & $27(55.1 \%)$ & $34(48.6 \%)$ & 0.504 \\
- DET & $22(44.9 \%)$ & $39.6(1.4)$ & 0.230 \\
Gestational age at birth (weeks) & $39.6(2.2)$ & $3(4.3 \%)$ & 0.166 \\
- Preterm birth (<37 weeks) & $1(2.0 \%)$ & $0(0 \%)$ & 0.072 \\
- Very preterm birth (<32 weeks) & $1(2.0 \%)$ & $3460.2(519.5)$ & 0.230 \\
Birthweight (grams) & $3301.4(529.5)$ & $1(1.4 \%)$ & 0.398 \\
- Low birthweight (<2500 g) & $4(8.2 \%)$ & $0(0 \%)$ & 0.339 \\
- Very low birthweight (<1500 g) & $1(2.0 \%)$ & $38(54.3 \%)$ & 0.351 \\
Breastfeeding (Yes) & $29(59.2 \%)$ & $3.8(7.8)$ & 0.693 \\
- Duration of breastfeeding (months) & $2.6(3.3)$ & $66(94.3 \%)$ & 0.501 \\
Performing sports (Yes) & $43(87.8 \%)$ & $2.9(1.9)$ & 0.253 \\
- Duration of sports/ week (hrs) & $2.8(1.8)$ & & \\
Medical history & & $5(7.1 \%)$ & $9(12.9 \%)$ \\
- Autism related disorders (c) & $2(4.1 \%)$ & & \\
- Allergic problems (including asthma, eczema) & $3(6.1 \%)$ & & \\
\hline
\end{tabular}

a. Categorical data presented as $n(\%)=$ Number of cases (percentage of cases)

b. Continuous data presented as mean (SD)

c. Autism related disorders: PDD-NOS (2x), ADD (1x), Autism (2x) and ADHD (2x). 
Table III - Baseline characteristics of parents of participating children (a)

\begin{tabular}{llll}
\hline Characteristics & Cook (n=49) ${ }^{(\mathrm{bc})}$ & Vitrolife $(\mathrm{n}=70)$ (bc) & P-value \\
\hline Maternal characteristics & & & \\
- Age (years) & $42.6(3.2)$ & $42.5(4.2)$ & 0.786 \\
- Height (cm) & $167.4(7.0)$ & $169.2(6.2)$ & 0.141 \\
- Weight (kg) & $68.8(10.5)$ & $73.1(12.4)$ & 0.129 \\
- BMl (kg/m²) & $24.5(3.6)$ & $25.5(3.8)$ & 0.309 \\
- Smoking (Yes) & $11(22.4 \%)$ & $6(8.6 \%)$ & 0.029 \\
- Higher Education & $21(42.9 \%)$ & $20(28.6 \%)$ & 0.089 \\
- Caucasian ethnicity & $45(91.8 \%)$ & $65(92.9 \%)$ & 0.613 \\
- Exercising regularly & $30(61.2 \%)$ & $40(57.1 \%)$ & 0.723 \\
- Primary infertility & $37(75.5 \%)$ & $54(77.1 \%)$ & 0.836 \\
Paternal characteristics & & & \\
- Age (years) & $45.8(5.2)$ & $45.0(4.6)$ & 0.285 \\
- Height (cm) & $180.3(7.8)$ & $182.8(7.8)$ & 0.089 \\
- Weight (kg) & $86.9(12.4)$ & $90.7(12.8)$ & 0.120 \\
- BMl (kg/m²) & $26.7(3.4)$ & $27.1(3.3)$ & 0.619 \\
- Smoking (Yes) & $6(12.2 \%)$ & $12(17 \%)$ & 0.307 \\
- Higher Education & $13(26.5 \%)$ & $31(44.3 \%)$ & 0.032 \\
- Caucasian ethnicity & $48(97.9 \%)$ & $68(97.1 \%)$ & 0.684 \\
- Exercising regularly & $28(57.1 \%)$ & $37(52.9 \%)$ & 0.557 \\
\hline
\end{tabular}

a. Baseline characteristics in this table represent characteristics at the time of the measurement (when the child was 9 years old).

b. Categorical data presented as $n(\%)=$ Number of cases (percentage of cases)

c. Continuous data presented as mean (SD) 
Figure 1: Results for effect of culture medium on cognitive development, using mixed effects multilevel analysis. For all domains, an unstructured matrix with random intercept was used. The graphs represent the results for the fixed effects of the multilevel analysis.
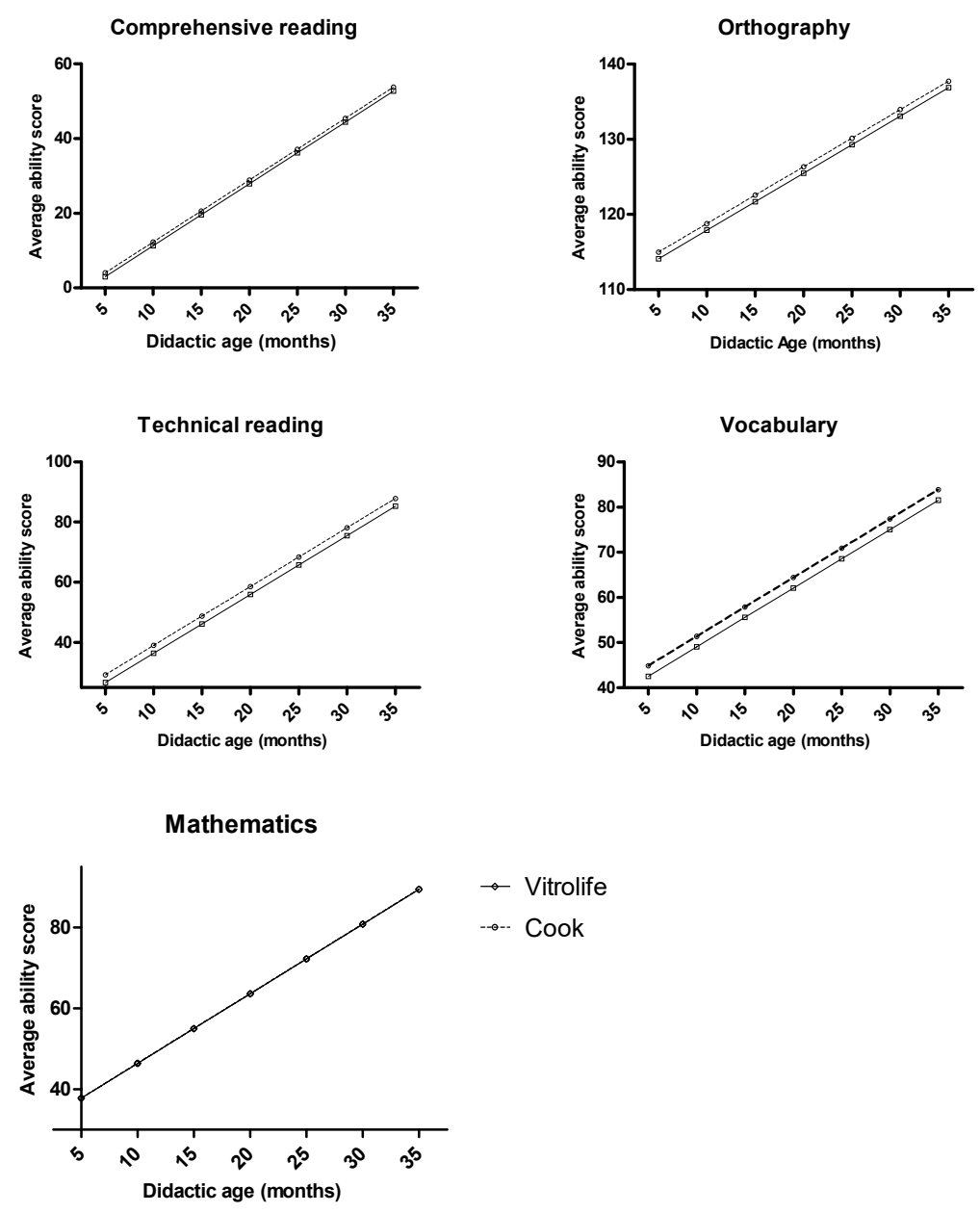

\begin{tabular}{|ll|l|l|}
\hline & $\begin{array}{l}\text { Adj. Beta Culture } \\
\text { Medium (ab) }\end{array}$ & $95 \% \mathrm{Cl}$ & Adj. P-value \\
\hline Comprehensive Reading & -1.328 & -5.60 to 2.94 & 0.539 \\
\hline Orthography & -0.861 & -2.94 to 1.22 & 0.413 \\
\hline Technical Reading & -1.900 & -6.94 to 3.14 & 0.456 \\
\hline Vocabulary & -2.586 & -8.48 to 3.31 & 0.318 \\
\hline Mathematics & 0.567 & -3.63 to 4.77 & 0.789 \\
\hline
\end{tabular}

a. Cook culture medium was used as the reference in this analysis. Therefore, the adjusted Beta reflects the estimated difference for the Vitrolife group compared to the Cook group. The presented confidence intervals reflect the $95 \% \mathrm{Cl}$ of the adjusted Beta.

b. Values are adjusted for educational level of the parents, gender of the child, age of the mother, smoking behavior of the mother, ethnicity of the parents and (duration of) being breastfed. 


\section{M5 Average compared to National average}

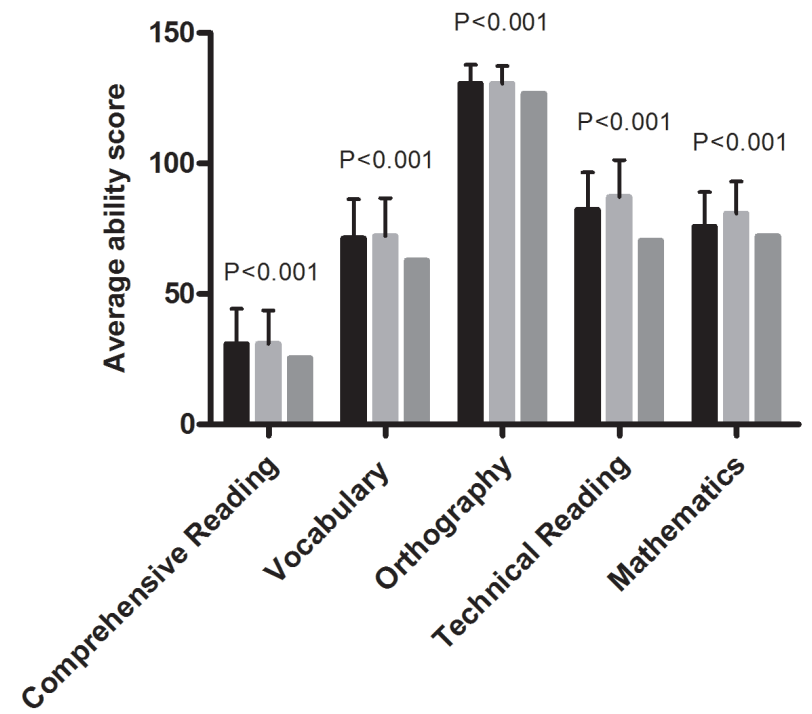

Cook

Vitrolife

National Average

Values are based on the true average value for the IVF groups compared to the true national average. Errorbars represent the standard deviation of the mean. P-values represent the statistical difference between the IVF children (both culture medium groups analysed as one group) and the national average. 


\section{DISCUSSION}

Comparison of all school performance (CITO) test scores of the children showed no significant differences between the two culture media groups. The results of our study are in contrast with the only other study published earlier on effects of culture medium on cognitive development. This study showed a lower general development and more developmental problems in children born after culture in SSM culture medium if compared to Global culture medium (Bouillon et al., 2016). However, the endpoint of our study is slightly different, since we focused specifically on school performance while the study by Bouillon et al. also compared motor development and social behavior. However, we checked for differences in major behavioral or developmental problems by asking the parents for the presence of attention deficit hyperactivity disorder or autism spectrum disorders, which was comparable between the two groups. Also the culture media used in the study of Bouillon and co-workers were different from the culture media we compared. Results of different studies focusing on cognitive development of IVF children compared to naturally conceived children have been published with conflicting results (Rumbold et al., 2017). Different study designs, the use of different test instruments and population compositions, and small population sizes have led to different outcomes in several studies comparing children born after ART with naturally conceived children.

A study by Spangmose et al. (2017) found lower cognitive scores for IVF singleton adolescents (15-16 years) if compared to naturally conceived controls using a large Danish administrative data register with standardized scores of school performance. This was not seen in twins (Spangmose et al., 2017). Another study performed by Barbuscia and Mills (2017) reported results of a comparable study, but found opposite results, with IVF children performing better than controls. At the ages of 3 and 5 years, children conceived with the aid of IVF had statistically higher verbal cognitive abilities than NC children. However, this difference consistently decreased over time and had disappeared by age 11 (Barbuscia and Mills, 2017).

Both groups in our study showed significantly higher academic performance if compared to the national average scores at the age of 9 years. Since it is known that parental education is a very important parameter determining the academic performance of the child (Chin-Lun Hung et al., 2015, Ruijsbroek et al., 2015, Spangmose et al., 2017), the most likely explanation for this difference is the higher level of parental education in our study population compared to the average Dutch population. The percentage of at least 1 higher educated parent was $45.9 \%$ for the Cook group and $45.3 \%$ for the Vitrolife group. This percentage was $35.2 \%$ for Dutch people from the same year of birth as the parents of the children from our cohort (CBS). Higher parental education in ART groups compared 
to a natural conception cohort is also described in several other studies (Barbuscia and Mills, 2017, Mains et al., 2010, Spangmose et al., 2017). Also our analyses showed that parental level of education was significantly associated with the ability scores of the child, which supports this theory.

Limitations of our study are the relatively low participation rate (47.7\%) (Zandstra et al. 2018) and hence the relatively small sample sizes. However, with the current sample size we were able to detect the clinically relevant difference in ability score between the 2 groups. The CITO tests are validated tests to monitor progression of children over time and to compare school performance with a child's peers. Therefore, it allows for an objective, validated comparison between the two culture medium groups and gave us the opportunity to compare these results with the average Dutch children in the same age groups. Although further research on long-term academic skills and behavior is essential, the results of this study are reassuring and should make parents of children born after IVF feel comfortable with their child's cognitive development.

In conclusion, our study did not show any significant differences in cognitive development if children born after culture as an embryo for 2-3 days in one of 2 different culture media were compared. Significant differences were observed if the scores of the IVF children were compared with the general Dutch population. The latter is most likely explained by a higher parental education level in the IVF group. 


\section{REFERENCES}

Barbuscia A, Mills MC. Cognitive development in children up to age 11 years born after ART-a longitudinal cohort study. Hum Reprod 2017;32: 1482-1488.

Barbuscia A, Mills MC. Cognitive development in children up to age 11 years born after ART-a longitudinal cohort study. Hum Reprod 2017: 1-7.

Bergh C, Wennerholm UB. Obstetric outcome and long-term follow up of children conceived through assisted reproduction. Best Pract Res Clin Obstet Gynaecol 2012;26: 841-852.

Berry KA, Baron IS, Weiss BA, Baker R, Ahronovich MD, Litman FR. In vitro fertilization and late preterm preschoolers' neuropsychological outcomes: the PETIT study. Am J Obstet Gynecol 2013;209: 356.e351-356.

Bouillon C, Léandri R, Desch L, Ernst A, Bruno C, Cerf C, Chiron A, Souchay C, Burguet A, Jimenez C et al. Does Embryo Culture Medium Influence the Health and Development of Children Born after In Vitro Fertilization? PLoS One 2016;11: e0150857.

CBS. https://www.onderwijsincijfers.nl/kengetallen/sectoroverstijgend/nederlands-onderwijsstelsel/hoogst-behaalde-onderwijsniveau.

Chin-Lun Hung G, Hahn J, Alamiri B, Buka SL, Goldstein JM, Laird N, Nelson CA, Smoller JW, Gilman SE. Socioeconomic disadvantage and neural development from infancy through early childhood. Int J Epidemiol 2015;44: 1889-1899.

CITO. 2016, http://www.onderwijsadvies.nl/media/889059/vaardigheidsscores-en-niveau-a-e-v8.pdf.

Dumoulin JC, Land JA, Van Montfoort AP, Nelissen EC, Coonen E, Derhaag JG, Schreurs IL, Dunselman GA, Kester $A D$, Geraedts JP et al. Effect of in vitro culture of human embryos on birthweight of newborns. Hum Reprod 2010;25: 605-612.

Jackson RA, Gibson KA, Wu YW, Croughan MS. Perinatal outcomes in singletons following in vitro fertilization: a meta-analysis. Obstet Gyneco/ 2004;103: 551-563.

Kleijkers SH, van Montfoort AP, Smits LJ, Viechtbauer W, Roseboom TJ, Nelissen EC, Coonen E, Derhaag JG, Bastings L, Schreurs IE et al. IVF culture medium affects post-natal weight in humans during the first 2 years of life. Hum Reprod 2014;29: 661-669.

Mains L, Zimmerman M, Blaine J, Stegmann B, Sparks A, Ansley T, Van Voorhis B. Achievement test performance in children conceived by IVF. Hum Reprod 2010;25: 2605-2611.

Matte TD, Bresnahan M, Begg MD, Susser E. Influence of variation in birth weight within normal range and within sibships on IQ at age 7 years: cohort study. BMJ 2001;323: 310-314.

Murray E, Pearson R, Fernandes M, Santos IS, Barros FC, Victora CG, Stein A, Matijasevich A. Are fetal growth impairment and preterm birth causally related to child attention problems and ADHD? Evidence from a comparison between high-income and middle-income cohorts. J Epidemiol Community Health 2016;70: 704-709.

Pinborg A, Wennerholm UB, Romundstad LB, Loft A, Aittomaki K, Söderström-Anttila V, Nygren KG, Hazekamp J, Bergh C. Why do singletons conceived after assisted reproduction technology have adverse perinatal outcome? Systematic review and meta-analysis. Hum Reprod Update 2013;19: 87-104.

Ruijsbroek A, Wijga AH, Gehring U, Kerkhof M, Droomers M. School Performance: A Matter of Health or Socio-Economic Background? Findings from the PIAMA Birth Cohort Study. PLoS One 2015;10: e0134780.

Rumbold AR, Moore VM, Whitrow MJ, Oswald TK, Moran LJ, Fernandez RC, Barnhart KT, Davies MJ. The impact of specific fertility treatments on cognitive development in childhood and adolescence: a systematic review. Hum Reprod 2017;32: 1489-1507.

Schendelaar P, La Bastide-Van Gemert S, Heineman MJ, Middelburg KJ, Seggers J, Van den Heuvel ER, Hadders-Algra M. Subfertility factors rather than assisted conception factors affect cognitive and behavioural development of 4-year-old singletons. Reprod Biomed Online 2016;33: 752-762. 
Spangmose AL, Malchau SS, Schmidt L, Vassard D, Rasmussen S, Loft A, Forman J, Pinborg A. Academic performance in adolescents born after ART-a nationwide registry-based cohort study. Hum Reprod 2017;32: 447-456.

Twilhaar ES, de Kieviet JF, Aarnoudse-Moens CS, van Elburg RM, Oosterlaan J. Academic performance of children born preterm: a meta-analysis and meta-regression. Arch Dis Child Fetal Neonatal Ed 2017.

Zandstra H, Brentjens L, Spauwen B, Touwslager RNH, Bons JAP, Mulder AL, Smits LJM, van der Hoeven M, van Golde RJT, Evers JLH et al. Association of culture medium with growth, weight and cardiovascular development of IVF children at the age of 9 years. Hum Reprod 2018.

Zandstra H, Van Montfoort AP, Dumoulin JC. Does the type of culture medium used influence birthweight of children born after IVF? Hum Reprod 2015;30: 2693. 


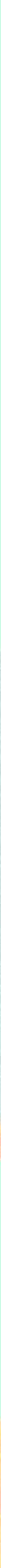




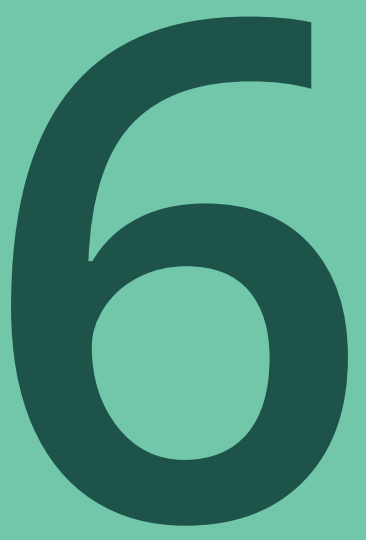

\section{Increased blood pressure and impaired endothelial function after accelerated growth in IVF/ICSI children}

Zandstra H, Van Montfoort APA, Dumoulin JCM, Zimmermann LJ, Touwslager RN Submitted September 2018 


\section{ABSTRACT}

\section{Study question}

In the present study, we aimed to analyze neonatal, infant and childhood growth simultaneously in a population of children born after assisted reproductive techniques. We aimed to study metabolic end-points; and to examine endothelial function. We hypothesized a harmful effect of accelerated growth on our cardiometabolic development.

\section{Summary answer}

Neonatal, infant and childhood growth are positively related to blood pressure in IVF/ ICSI offspring. Growth in childhood was negatively associated with endothelial function.

\section{What is known already?}

Offspring of pregnancies conceived after assisted reproductive techniques (ART) are at risk for later cardiometabolic risk factors. It is well established that early growth is related to numerous later cardiometabolic risk factors (DOHaD concept), for example high blood pressure.

\section{Study Design, Size and Duration}

The relationship between early growth and later cardiometabolic risk profile was studied in the MEDIUM-KIDS study131 children (48.1\% males) were studied at the average age of 9.4 years.

\section{Participants/Materials, Settings and Methods}

This is a prospective observational cohort study in children born after an in vitro fertilization/ intracytoplasmic sperm injection (IVF/ ICSI) treatment. All participants were healthy 9-year-old children, born at term. Growth was defined as z-score difference in weight. From one month onwards the models were always adjusted for difference in height z-score. The following growth windows were studied simultaneously: 0-1 month, 1-3 months, 3-6 months, 6-11 months, 11-24 months and 2-6 years. Blood pressure, skinfolds, lipid spectrum, hair cortisone, glucose and insulin were measured. Maximum skin perfusion after transdermal delivery of acetylcholine as a measure of endothelial function was collected. 


\section{Main results and the role of chance}

In multivariable linear regression analyses, multiple growth windows were positively associated with blood pressure, for example growth from 2-6 years: $B=4.13, P=0.005$. Maximum skin perfusion after acetylcholine was negatively associated with height-adjusted weight gain from 2-6 years: $B=-0.09$ (log scale), $P=0.03$. Several growth windows were positively linked with total adiposity. Lipids, glucose tolerance indices and cortisone were not related to growth. In conclusion, neonatal, infant and childhood growth are positively related to blood pressure in IVF/ ICSI offspring. Growth in childhood was negatively associated with endothelial function. For reasons of generalizability, we only included healthy children, born at term in this study.

\section{Limitations, reasons for caution}

This study is of modest size, of observational nature and we did not include a control group. Therefore we cannot assess whether the observed associations are causal. It is also not possible to analyze if our observations are specific for, or exacerbated in the ART population. Ideally, a control group of naturally conceived siblings of IVF/ ICSI children should simultaneously be studied to address the previous and to assess the impact of the ART procedure without the influence of parental (subfertility) characteristics.

\section{Wider implications of the findings}

This is an important step in understanding the reported increased risk for hypertension in ART-offspring. 


\section{INTRODUCTION}

Offspring of assisted reproductive techniques (ART) conceived pregnancies have an increased risk for several cardiometabolic risk factors. These associations probably originate through epigenetic mechanisms in the early embryonic stage (Fleming et al., 2018, Vrooman and Bartolomei, 2017). This fits into the developmental origins of health and disease (DOHaD) concept, which states that early life aspects have lifelong health implications. In in vitro fertilization (IVF) or intracytoplasmic sperm injection (ICSI) treatments, the fertilization of the oocyte and early embryo development occur outside the human body and therefore under non-natural circumstances. These processes coincide with epigenetic reprogramming events within the gametes and embryo. During the IVF/ ICSI procedure several environmental variables, such as embryo culture medium, may impact epigenetic reprogramming.

From a vascular perspective, children born after ART display endothelial dysfunction and vascular stiffness compared to controls of similar (and normal) gestational age and birth weight (Scherrer et al., 2012). Furthermore, persistent early cardiac remodeling (Valenzuela-Alcaraz et al., 2013) and increased blood pressure were identified (Ceelen et al., 2008, Sakka et al., 2010, Valenzuela-Alcaraz et al., 2013). Metabolic changes have also been found in ART children. Compared to naturally conceived controls fasting glucose levels were higher (Ceelen et al., 2008), as well as triglycerides (Sakka et al., 2010). A recent meta-analysis showed increased blood pressure, higher fasting insulin levels but a slightly more favorable lipid profile in ART offspring (Guo et al., 2017).

From a growth perspective, ART newborns have an increased risk for premature birth and for low birth weight (Pandey et al., 2012). One would expect that relatively low birth weight would be followed by relative fast infant growth (regression to the mean), like was shown in a study by Ceelen et al., at least in late infancy (Ceelen et al., 2009). On the contrary, in another study no rapid weight gain was seen in ART singletons in the first year and no differences were seen in any growth parameter in the first 3 years (Yeung et al., 2016). Also, normal growth in IVF and ICSI children up to 12 years old was reported in a paper by Basatemur and colleagues (Basatemur et al., 2010). Altogether, it remains unclear whether ART (the procedure itself, parental subfertility characteristics, or an indirect effect of the ART procedure on birth weight) predisposes for altered growth in infancy and childhood.

It is well established that early growth is related to numerous later cardiometabolic risk factors (DOHaD concept), for example high blood pressure (Kagura et al., 2016, Taine et al., 2016). There is an ongoing debate about the exact timing of these effects, but early postnatal growth (<3 months) has consistently been linked to later cardiometabolic risk 
factors (Fabricius-Bjerre et al., 2011, Khuc et al., 2012, Leunissen et al., 2009). Ceelen et al. analyzed the effects of postnatal growth in IVF children (Ceelen et al., 2009). They found early childhood growth, but not infant growth, to be positively related to later blood pressure (only in IVF) and skinfold thicknesses (both IVF and controls).

In the present study, we aimed to analyze neonatal, infant and childhood growth simultaneously; to study metabolic end-points; and to examine endothelial function. We hypothesized a harmful effect of accelerated growth on our outcome parameters. For reasons of generalizability, we only included healthy children, born at term. 


\section{METHODS}

\section{Participants}

Parents of all singletons born after an IVF treatment with fresh embryo transfer at Maastricht University Medical Centre between July 2003 and December 2006 were approached to participate in the MEDIUM-KIDS study. In this prospective observational cohort study 9-year-old children were investigated with regard to cardiometabolic risk profile in relation to IVF culture medium (Cook and Vitrolife) (Dumoulin et al., 2010). 47.7\% of the potential participants gave informed consent $(n=136)$. The baseline characteristics at the time of birth of the participating children were comparable to those of the children not participating in the study (Zandstra et al., 2018). Pregnancy and neonatal characteristics were retrieved from the hospital charts. Gestational age was determined by the following formula: (date of birth - oocyte retrieval date) + 14 days. Prematurely born children $(<37$ weeks gestational age) were excluded from the analysis ( $n=5)$. Two mothers had diabetes of pregnancy. Eight mothers had a hypertensive disorder during pregnancy (one HELLP -i.e., Hemolysis, Elevated Liver enzymes, Low Platelet count-, one pre-eclampsia and six gestational hypertension, defined as clinical diagnosis in the hospital charts). There were no relevant birth defects or later diagnosed illnesses.

Details of the IVF procedures were published previously, as the MEDIUM-KIDS study was part of an earlier study (Dumoulin et al., 2010). Only singletons born after transfer of fresh embryos were included.

\section{Setting}

Between March 2014 and December 2016 the participants were invited for a 2.5 hours visit to our hospital. Two experienced researchers performed the tests. Information on possible confounders was recorded by a questionnaire completed by the parents during the visit.

\section{Collection of independent variables}

Growth data were received electronically from child welfare centers, which offer free consultations and vaccinations to all Dutch children. At these centers length and weight are recorded. In the present study we analyzed the data obtained at the approximate ages of 1, 3, 6, 11, 24 and 72 months. The obtained values were converted into sex and age specific z-scores by an online calculator (https://groeiweb.pgdata.nl/calculator.asp), by use of data from the Dutch $3^{\text {rd }}$ national growth study (weight) and the Dutch $5^{\text {th }}$ national growth study (height) (Schonbeck et al., 2013, Schönbeck et al., 2011). Birth length was missing in $50.4 \%$ of the cases and was therefore not analyzed. Birth weight was collected from the hospital charts and converted into z-scores by use of sex and parity specific reference 
data accurate to a gestational age of one day (Visser et al., 2009). Growth was defined as difference in weight z-scores. The following growth windows were studied: 0-1 month, 1-3 months, 3-6 months, 6-11 months, 11-24 months and 2-6 years.

Maternal pre-pregnancy weight and height were retrieved from hospital charts and pre-pregnancy BMI was calculated. Highest completed maternal education level was dichotomized; high education level being defined as the completion of higher professional education or university. Breast feeding was reported by the parents and dichotomized (yes/ no). Maternal smoking during pregnancy was self-reported and dichotomized (yes/ no).

\section{Measurement of dependent variables}

Children were measured lightly clothed; height (to the nearest $0.1 \mathrm{~cm}$ ) and body weight (to the nearest $0.1 \mathrm{~kg}$ ) were measured using a stadiometer (DGI 250D, The Netherlands) and scale (Seca 704, Germany). Skinfold thicknesses (triceps, biceps, subscapular and suprailiac) were measured in triplicate on the non-dominant side of the body by a Harpenden skinfold caliper (Baty International, United Kingdom). Total adiposity was calculated as the sum of the four averaged skinfold thicknesses. The sum of triceps and biceps skinfolds represented peripheral adiposity and the sum of subscapular and suprailiac skinfolds represented truncal adiposity.

Systolic and diastolic blood pressures were measured in triplicate on the non-dominant arm while the child was sitting after a minimum of 30 minutes' rest in supine position on a bed. It was measured using a calibrated automatic device (Accutorr Plus, Datascope Inc., Montvale, New Jersey, USA) and averaged for analysis. All but 26 children gave permission for a venipuncture. Participants who did not fast overnight were excluded from the analyses in blood samples ( $n=6)$. Five participants had incomplete results because not enough blood could be drawn. Fasting plasma glucose was measured using an enzymatic colorimetric assay (Cobas 8000 instrument, Roche Diagnostics, Mannheim, Germany). Fasting insulin was measured in serum using an immunometric assay (XPi instrument, Siemens Medical Solutions Diagnostics, LA). HOMA-IR was calculated by the following formula: ((insulin (pmol/l)/ 6.94)*glucose (mmol/l))/ 22.5. Total cholesterol, HDL cholesterol and triglycerides were determined using an enzymatic colorimetric assay (Cobas 8000 instrument, Roche Diagnostics, Mannheim, Germany). LDL was calculated by the Friedewald formula.

Hair samples were collected when the child had hair $>3 \mathrm{~cm}$ and when parents consented. The hair strands ( $\sim 3 \mathrm{~mm}$ in diameter) were collected from the posterior vertex region, as close to the scalp as possible. Cortisone was measured by use of chromatography of the $3 \mathrm{~cm}$ hair segment closest to the scalp. 
Laser-Doppler flowmetry of the forearm was performed to assess vascular function (Periflux 5000 system, Perimed A.B., Sweden). By use of iontophoresis acetylcholine and nitroprusside were administered trans dermally (Perilont system; Perimed A.B., Sweden) and vasodilatation curves were constructed, as described previously (Touwslager et al., 2012). Acetylcholine induces endothelium-dependent vasodilatation via stimulation of the endothelium, while nitroprusside induces endothelium-independent vasodilatation as a direct nitric oxide donor. Maximum perfusion was recorded in perfusion units. The recordings were analyzed by H.Z. and R.T, who were blinded to the growth patterns and reached consensus about quality criteria such as movement artifacts.

\section{Ethical approval}

The study was registered in the Dutch Trial register (trial number NTR4220) and the local ethics committee approved the study. Both parents of all children gave written informed consent.

\section{Statistical analysis}

Insulin values below the detection limit of $12 \mathrm{pmol} / \mathrm{I}$ were analyzed as $12 \mathrm{pmol} / \mathrm{l}$. Hair cortisone values below the detection limit of $0.68 \mathrm{pmol} / \mathrm{mg}$ hair were similarly analyzed as $0.68 \mathrm{pmol} / \mathrm{mg}$ hair. In case of a significant Kolmogorov-Smirnov test, a visual inspection of a variable was performed and a 10 log transformation was performed when necessary. Linear regression analysis was used as the primary statistical analysis method. All growth windows were analyzed simultaneously in one model to take regression to the mean into account. Model 1 was constructed with only weight gain as the predicting variable. The model was adjusted for birth weight z-score and age at measurement of the outcome variable. Model 2 was additionally adjusted for maternal pre-pregnancy BMI, culture medium, maternal smoking during pregnancy, maternal hypertension during pregnancy, maternal education level and breast feeding. Model 3 equals model 2, but was further adjusted for height growth (starting from 1 month), so the results for weight gain can be interpreted as being independent of gain in height. In model 3, adjustments were made for height at day of measurement in the blood pressure models and for baseline perfusion in the maximum skin perfusion models. To avoid collider bias or over adjustment, we did not correct our models for current weight or current BMI (Chiolero et al., 2012, Woo, 2017). We considered a $P$-value $<0.05$ to be statistically significant. All analyses were performed by use of IBM SPSS statistics 23. 


\section{RESULTS}

The baseline characteristics of the study sample are shown in table I. Gestational age and birth weight were normal compared to the general population.

Growth from 0-1 month, 3-6 months and 2-6 years was positively associated with systolic blood pressure at 9 years in the final height-adjusted models, for example growth from 2-6 years: $B=4.13, P=0.005$ (table $I$ ), indicating a $4.13 \mathrm{mmHg}$ rise in blood pressure per one unit increase in weight z-score between two and six years. Growth in the first month was also positively related to diastolic blood pressure: $B=4.26, P=0.004$.

In a paired samples t-test, there was no difference between baseline perfusion in the acetylcholine and nitroprusside protocols $(P=0.96)$. The correlation between baseline perfusion in the acetylcholine and nitroprusside protocols was $0.71(P<0.001)$. Maximum perfusion in response to acetylcholine was negatively related to growth from 2-6 years: $\mathrm{B}=-0.09$ (log scale), $\mathrm{P}=0.03$. This effect size corresponds to a $19.5 \%$ decrease in maximum perfusion per one unit increase in weight z-score. There were no significant associations between growth and maximum perfusion after nitroprusside, e.g. model 3, growth from 2-6 years: $B=-0.03$ (log scale), $P=0.38$.

Total adiposity was positively related to growth in all age windows, except 0-1 month, for example growth from 11-24 months: $B=0.13$ (log scale), $P<0.001$. This effect size corresponds to a $40.3 \%$ increase in total adiposity per one unit increase in weight z-score. In model 1, truncal:peripheral adiposity ratio was positively related to growth from 11-24 months: $B=0.05$ (log scale), $P=0.04$, this association was no longer significant in model 2 and 3 (data not shown).

No significant relationships were identified between growth and cortisone, HOMA-IR, insulin, glucose, triglycerides, total cholesterol:HDL ratio or LDL (data not shown). 
Table I - Baseline characteristics of the study population

\begin{tabular}{|c|c|c|c|c|}
\hline & $\mathrm{n}$ & Mean (SD) & Median (IQR) & $\%$ \\
\hline \multicolumn{5}{|l|}{ General } \\
\hline Males & 131 & & & 48,1 \\
\hline High maternal education & 131 & & & 38,2 \\
\hline Maternal prepregnancy BMI & 131 & $24,47(3,15)$ & & \\
\hline \multicolumn{5}{|l|}{ Obstetric } \\
\hline Gestational age (weeks) & 131 & $39,83(1,20)$ & & \\
\hline Birth weight (SD) & 131 & $-0,02(0,99)$ & & \\
\hline Received breastfeeding & 131 & & & 55,7 \\
\hline Gestational hypertension & 131 & & & 6,1 \\
\hline Smoking during pregnancy & 131 & & & 5,3 \\
\hline \multicolumn{5}{|l|}{ ART } \\
\hline Cook medium & 131 & & & 45,0 \\
\hline Maternal age (years) & 131 & $32,92(3,74)$ & & \\
\hline ICSI & 131 & & & 64,9 \\
\hline \multicolumn{5}{|l|}{ Growth } \\
\hline Age at measurement '1 month' (days) & 123 & $29,46(5,67)$ & & \\
\hline Age at measurement ' 3 months' (days) & 123 & $93,19(10,77)$ & & \\
\hline Age at measurement ' 6 months' (days) & 122 & $181,02(12,76)$ & & \\
\hline Age at measurement '11 months' (days) & 127 & $345,11(18,28)$ & & \\
\hline Age at measurement '24 months' (years) & 123 & $2,09(0,11)$ & & \\
\hline Age at measurement '6 years' (years) & 121 & $5,88(0,52)$ & & \\
\hline 0-1 month ( $\Delta$ weight z-score) & 123 & $0,05(0,81)$ & & \\
\hline 1-3 months ( $\triangle$ weight z-score) & 123 & $0,37(0,75)$ & & \\
\hline 3-6 months ( $\Delta$ weight z-score) & 122 & $-0,08(0,63)$ & & \\
\hline 6-11 months ( $\triangle$ weight z-score) & 122 & $-0,18(0,61)$ & & \\
\hline 11-24 months ( $\Delta$ weight z-score) & 122 & $-0,28(0,66)$ & & \\
\hline 2-6 years ( $\Delta$ weight z-score) & 110 & $0,20(0,76)$ & & \\
\hline 1-3 months ( $\triangle$ length z-score) & 110 & $0,44(0,56)$ & & \\
\hline 3-6 months ( $\Delta$ length z-score) & 112 & $-0,03(0,50)$ & & \\
\hline 6-11 months ( $\triangle$ length z-score) & 117 & $0,02(0,51)$ & & \\
\hline 11-24 months ( $\Delta$ length z-score) & 119 & $-0,07(0,68)$ & & \\
\hline 2-6 years ( $\Delta$ length $z$-score) & 113 & $-0,24(0,54)$ & & \\
\hline \multicolumn{5}{|l|}{ Cardiometabolic } \\
\hline Age on measurement day (years) & 131 & & $9,43(0,33)$ & \\
\hline BMI on measurement day (kg/m2) & 131 & $17,14(2,45)$ & & \\
\hline Baseline perfusion acetylcholine protocol (PU) & 121 & & $3,73(8,60)$ & \\
\hline Maximum perfusion acetylcholine protocol (PU) & 121 & & $80,36(60,69)$ & \\
\hline Baseline perfusion nitroprusside protocol (PU) & 115 & & $3,65(8,22)$ & \\
\hline Maximum perfusion nitroprusside protocol (PU) & 115 & & $101,51(69,04)$ & \\
\hline
\end{tabular}


Table I - Continued

\begin{tabular}{|c|c|c|c|c|}
\hline & $\mathrm{n}$ & Mean (SD) & Median (IQR) & $\%$ \\
\hline \multicolumn{5}{|l|}{ Cardiometabolic } \\
\hline Systolic blood pressure (mmHg) & 131 & $100,38(7,43)$ & & \\
\hline Diastolic blood pressure $(\mathrm{mmHg})$ & 131 & $60,05(6,70)$ & & \\
\hline Sum of skinfolds (mm) & 131 & & $33,66(26,53)$ & \\
\hline Total: truncal adiposity ratio & 131 & & $0,60(0,22)$ & \\
\hline Insulin (pmol/l) & 96 & & $30,65(28,70)$ & \\
\hline Glucose (mmol/l) & 93 & $4,71(0,34)$ & & \\
\hline HOMA IR & 91 & & $0,94(0,94)$ & \\
\hline Total:HDL cholesterol ratio & 97 & & $2,47(0,86)$ & \\
\hline Triglycerides (mmol/l) & 97 & & $0,63(0,41)$ & \\
\hline $\operatorname{LDL}(\mathrm{mmol} / \mathrm{l})$ & 97 & $2,31(0,56)$ & & \\
\hline Cortisone (pg/mg hair) & 116 & $12,68(7,23)$ & & \\
\hline
\end{tabular}

$S D=$ standard deviation, $I Q R=$ interquartile range, $B M I=$ body mass index, $A R T=$ assisted reproductive technique, ICSI= intracytoplasmic sperm injection, $\Delta=$ delta, $P U=$ perfusion units, HOMA IR = homeostatic model assessment, insulin resistance, $H D L=$ high density lipoprotein, $L D L=$ low density lipoprotein 
Table II - Regression coefficients for growth

\begin{tabular}{|c|c|c|c|c|c|c|c|c|c|}
\hline & \multicolumn{3}{|c|}{ Model 1} & \multicolumn{3}{|c|}{ Model 2} & \multicolumn{3}{|c|}{ Model 3} \\
\hline & $\mathrm{n}$ & B & $P$ & $\mathrm{n}$ & B & $P$ & $\mathrm{n}$ & B & $P$ \\
\hline \multicolumn{10}{|l|}{ Systolic blood pressure (mmHg) } \\
\hline$\Delta$ weight z-score 0-1 month & 103 & 3,58 & $0,001 *$ & 103 & 3,54 & $0,002 *$ & 85 & 5,51 & 0,001 * \\
\hline$\Delta$ weight z-score 1-3 months & & 1,65 & 0,15 & & 1,91 & 0,12 & & 3,71 & 0,05 \\
\hline$\Delta$ weight z-score 3-6 months & & 3,58 & $0,006^{*}$ & & 3,26 & $0,02 *$ & & 4,69 & $0,01 *$ \\
\hline$\Delta$ weight z-score 6-11 months & & 3,53 & $0,02 *$ & & 3,79 & $0,01 *$ & & 3,78 & 0,07 \\
\hline$\Delta$ weight z-score 11-24 months & & 1,93 & 0,09 & & 2,03 & 0,08 & & 0,42 & 0,80 \\
\hline$\Delta$ weight z-score $2-6$ years & & 3,71 & $<0,001^{*}$ & & 3,62 & $0,001^{*}$ & & 4,13 & $0,005^{*}$ \\
\hline \multicolumn{10}{|l|}{ Diastolic blood pressure ( $\mathrm{mmHg}$ ) } \\
\hline$\Delta$ weight z-score 0-1 month & 103 & 1,62 & 0,11 & 103 & 1,41 & 0,18 & 85 & 4,26 & $0,004 *$ \\
\hline$\Delta$ weight z-score 1-3 months & & $-0,74$ & 0,50 & & $-1,17$ & 0,32 & & 0,06 & 0,97 \\
\hline$\Delta$ weight z-score 3-6 months & & 1,69 & 0,17 & & 1,13 & 0,38 & & 2,92 & 0,08 \\
\hline$\Delta$ weight z-score 6-11 months & & 0,65 & 0,65 & & 0,98 & 0,50 & & 1,13 & 0,54 \\
\hline$\Delta$ weight z-score $11-24$ months & & 0,11 & 0,92 & & 0,20 & 0,86 & & $-0,29$ & 0,84 \\
\hline$\Delta$ weight z-score $2-6$ years & & 0,51 & 0,60 & & 0,48 & 0,63 & & 1,13 & 0,37 \\
\hline \multicolumn{10}{|c|}{ Maximum perfusion after acetylcholine (log PU) } \\
\hline$\Delta$ weight z-score 0-1 month & 94 & 0,03 & 0,52 & 94 & 0,03 & 0,48 & 78 & 0,01 & 0,84 \\
\hline$\Delta$ weight z-score 1-3 months & & 0,01 & 0,88 & & 0,01 & 0,86 & & 0,00 & 0,97 \\
\hline$\Delta$ weight z-score 3-6 months & & 0,03 & 0,61 & & 0,03 & 0,60 & & $-0,01$ & 0,86 \\
\hline$\Delta$ weight z-score 6-11 months & & 0,01 & 0,92 & & 0,02 & 0,77 & & 0,04 & 0,53 \\
\hline$\Delta$ weight z-score 11-24 months & & 0,03 & 0,50 & & 0,04 & 0,40 & & $-0,01$ & 0,85 \\
\hline$\Delta$ weight z-score $2-6$ years & & $-0,02$ & 0,57 & & $-0,02$ & 0,64 & & $-0,09$ & $0,03 *$ \\
\hline \multicolumn{10}{|l|}{ Total adiposity (log mm) } \\
\hline$\Delta$ weight z-score 0-1 month & 103 & 0,03 & 0,27 & 103 & 0,03 & 0,24 & 85 & 0,03 & 0,35 \\
\hline$\Delta$ weight z-score 1-3 months & & 0,05 & $0,03 *$ & & 0,07 & $0,009 *$ & & 0,09 & $0,01 *$ \\
\hline$\Delta$ weight z-score 3-6 months & & 0,08 & $0,002^{*}$ & & 0,09 & $0,002 *$ & & 0,12 & $0,001 *$ \\
\hline$\Delta$ weight z-score 6-11 months & & 0,06 & 0,06 & & 0,06 & 0,06 & & 0,10 & $0,01 *$ \\
\hline$\Delta$ weight z-score 11-24 months & & 0,08 & $0,001 *$ & & 0,08 & $0,001 *$ & & 0,13 & $<0,001^{*}$ \\
\hline$\Delta$ weight z-score 2-6 years & & 0,13 & $<0,001 *$ & & 0,14 & $<0,001 *$ & & 0,15 & $<0,001 *$ \\
\hline
\end{tabular}

Model 1: adjusted for age at measurement day, birth weight z-score and weight gain in the other time windows

Model 2: model 1 further adjusted for maternal pre-pregnancy BMI, culture medium, maternal smoking during pregnancy, maternal hypertension during pregnancy, maternal education level and breast feeding

Model 3: model 2 further adjusted for height growth in all windows except 0-1 month. Blood pressure models were adjusted for height, the perfusion model was adjusted for baseline perfusion

$\mathrm{PU}=$ perfusion units

* Indicates a significant association with $P<0.05$ 


\section{DISCUSSION}

In this study, we showed that in 9-year-old children born after IVF/ ICSI, weight gain from 0-1 month was positively related to both systolic and diastolic blood pressure. Moreover, height-adjusted weight gain from 3-6 months and 2-6 years were positively related to systolic blood pressure. We report clinically relevant effect sizes of 4-5.5 $\mathrm{mmHg}$ per one unit increase in weight z-score. The association between growth in early childhood and systolic blood pressure is in line with a previous study in IVF children (Ceelen et al., 2009), although we now add the fact that also infant growth, particularly growth from 0-1 month, is an influential growth window. Therefore, we cannot support a healthy infant growth trajectory in IVF/ ICSI children, as postulated by Ceelen et al. Although, it should be noted that our population had somewhat higher (and normal) birth weight than the population analyzed by Ceelen et al. and that our population was born at term (Ceelen et al., 2009).

To our knowledge, we are the first to describe the inverse association between growth in childhood, and maximum perfusion in response to acetylcholine in ART offspring. No relationships were shown between growth and maximum perfusion in response to nitroprusside. Therefore, it can be concluded that our results reflect endothelial dysfunction. Endothelial dysfunction precedes atherosclerosis and can be tested by acetylcholine induced vasodilatation (Puissant et al., 2013). The identification of a relationship between growth and endothelial function is consistent with a study in non-ART individuals, although this study analyzed growth in the first two weeks (Singhal et al., 2004) and to a previous study in our hospital in non-ART children up to two years of age, although based on the latter study we expected early infant growth to be of importance (Touwslager et al., 2015). Perfusion measurement by laser-Doppler was used because of the suitability to use this technique in children. This can however be challenging in children because of movement artifacts and the high variability (Puissant et al., 2013) in these measurements. We have shown a good interobserver variability of the obtained signal earlier (Touwslager et al., 2012) and the high correlation and equality between baseline perfusion in both arms was reassuring. The exact clinical importance of maximum skin perfusion after acetylcholine at 9 years of age is not known, but a 19.5\% decrease per one unit increase in z-score appears to be a reasonably large effect size.

Offspring of ART pregnancies is at increased risk for high blood pressure (Guo et al., 2017), possibly through eNOS -endothelial nitric oxide synthase- methylation changes (Rimoldi et al., 2014). ART offspring also displays endothelial dysfunction (Scherrer et al., 2012). In turn, endothelial dysfunction plays a role in the development of hypertension (Brandes, 2014). Earlier it was shown that accelerated growth in early childhood in IVF children was associated with an increase in blood pressure, but this was not the case 
in spontaneously conceived controls (Ceelen et al., 2009). Combining the previous with our results, we speculate that early accelerated growth may be involved in the reported increased risk for hypertension in ART offspring, with endothelial dysfunction as a possible underlying mechanism. Accelerated growth could be a 'second' hit on an already increased risk for hypertension and endothelial dysfunction in ART offspring. Importantly, nearly none of the studies identifying increased blood pressure in ART offspring controlled for growth, so growth could also be the 'only' hit. Alternatively, ART offspring may be extra susceptible to the deleterious effects of accelerated growth. Based on our study and the existing literature we recommend cardiometabolic follow-up for fast growing individuals after ART conceived pregnancies: both for reasons of prevention as for possible pharmacological (for example antioxidants) interventions in the future (Rimoldi et al., 2015).

Growth in all windows except 0-1 month was positively related to total sum of skinfolds. This was not surprising since we defined growth as difference in weight z-score and adjusted the analyses for difference in height z-score. Our results were in agreement with a previous study in IVF children (Ceelen et al., 2009). Extensive evidence links early growth to later obesity in non-ART individuals (Baidal et al., 2016). We did not identify a relationship between growth and an unfavorable ratio of truncal to peripheral adiposity.

Importantly, the identified associations were shown in a term, healthy, normal birth weight group, which was prospectively collected. Cardiometabolic effects of prematurity, a complication of IVF, could therefore be excluded. We did not perform an analysis to assess individual accelerated growth, but from the average differences in z-score in each growth window can be expected that growth was likely normal compared to the Dutch population. This is in line with the study by Yeung et al. which did not observe any risk for accelerated growth in the first year in ART children, rapid weight gain being defined as $>0.50$ SD (Yeung et al., 2016). This apparently normal growth is important for reasons of generalizability (both towards the majority of ART conceived offspring as to the general population), which we consider to be good in our study. Given our simultaneous analyses of all infant growth windows and concurrent analysis of height gain in model 3, our growth measures can be interpreted independently of each other and as weight gain in excess of what can be expected based on height gain. Although fast growth has beneficial short term effects, such as resistance to infections, there are no clear long-term neurodevelopmental benefits of fast growth for healthy term children (Belfort and Gillman, 2013). However, small-for-gestational-age term children have been shown to possibly benefit from a certain degree of catch-up growth in terms of later IQ, although height was not accounted for in this study (Lei et al., 2015). Optimal growth trajectories remains to be elucidated 
(Singhal, 2017), especially for ART offspring. Promising interventions for prevention of fast infant growth include breastfeeding (Carling et al., 2015), modification of protein content of formula (Patro-Golab et al., 2016) and responsive parenting intervention programs (Savage et al., 2016).

This study is of modest size, of observational nature and we did not include a control group. Therefore we cannot assess whether the observed associations are causal. It is also not possible to analyze if our observations are specific for, or exacerbated in the ART population. Next, we are unable to report whether ART children have altered growth patterns. A possible increased risk for cardiovascular risk factors in ART offspring, regardless of growth, can furthermore not be concluded. Ideally, a control group of naturally conceived siblings of IVF/ ICSI children should simultaneously be studied to address the previous and to assess the impact of the ART procedure without the influence of parental (subfertility) characteristics. Birth length availability was poor, so we could not adjust weight gain in the first months for gain in length. However, it should be noted that birth length measurements are not reliable (Johnson et al., 1998).

Neonatal, infant and childhood growth are positively related to blood pressure in IVF/ ICSI offspring. Growth in childhood was negatively associated with endothelial function. This is an important step in understanding the increased risk for hypertension in ART-offspring. 


\section{REFERENCES}

Baidal JAW, Locks LM, Cheng ER, Blake-Lamb TL, Perkins ME, Taveras EM. Risk Factors for Childhood Obesity in the First 1,000 Days A Systematic Review. Am J Prev Med 2016;50: 761-779.

Banrezes B, Sainte-Beuve T, Canon E, Schultz RM, Cancela J, Ozil JP. Adult body weight is programmed by a redox-regulated and energy-dependent process during the pronuclear stage in mouse. PLoS One 2011;6: e29388.

Barbuscia A, Mills MC. Cognitive development in children up to age 11 years born after ART-a longitudinal cohort study. Hum Reprod 2017: 1-7.

Barker DJ. Maternal nutrition, fetal nutrition, and disease in later life. Nutrition 1997;13: 807-813.

Barker DJ. Fetal origins of cardiovascular disease. Ann Med 1999;31 Suppl 1: 3-6.

Barker DJ. In utero programming of cardiovascular disease. Theriogenology 2000;53: 555-574.

Barker DJ. Adult consequences of fetal growth restriction. Clin Obstet Gynecol 2006;49: 270-283.

Barker DJP. The origins of the developmental origins theory. J Intern Med 2007;261: 412-417.

Basatemur E, Shevlin M, Sutcliffe A. Growth of children conceived by IVF and ICSI up to 12years of age. Reprod Biomed Online 2010;20: 144-149.

Bay B, Mortensen EL, Kesmodel US. Is subfertility or fertility treatment associated with long-term growth in the offspring? A cohort study. Fertil Steril 2014;102: 1117-1123.

Belfort MB, Gillman MW. Healthy infant growth: what are the trade-offs in the developed world? Nestle Nutr Inst Workshop Ser 2013;71: 171-184.

Belva F, Henriet S, Liebaers I, Van Steirteghem A, Celestin-Westreich S, Bonduelle M. Medical outcome of 8-year-old singleton ICSI children (born >or=32 weeks' gestation) and a spontaneously conceived comparison group. Hum Reprod 2007;22: 506-515.

Belva F, Painter R, Bonduelle M, Roelants M, Devroey P, De Schepper J. Are ICSI adolescents at risk for increased adiposity? Hum Reprod 2012;27: 257-264.

Bergh C, Wennerholm UB. Obstetric outcome and long-term follow up of children conceived through assisted reproduction. Best Pract Res Clin Obstet Gynaecol 2012;26: 841-852.

Berry KA, Baron IS, Weiss BA, Baker R, Ahronovich MD, Litman FR. In vitro fertilization and late preterm preschoolers neuropsychological outcomes: the PETIT study. Am J Obstet Gynecol 2013;209: 356.e351-356.

Bloise E, Feuer SK, Rinaudo PF. Comparative intrauterine development and placental function of ART concepti: implications for human reproductive medicine and animal breeding. Human reproduction update 2014;20: 822-839.

Bloise E, Lin W, Liu X, Simbulan R, Kolahi KS, Petraglia F, Maltepe E, Donjacour A, Rinaudo P. Impaired placental nutrient transport in mice generated by in vitro fertilization. Endocrinology 2012;153: 3457-3467.

Boivin J, Bunting L, Collins JA, Nygren KG. International estimates of infertility prevalence and treatment-seeking: potential need and demand for infertility medical care. Hum Reprod 2007;22: 1506-1512.

Bouillon C, Léandri R, Desch L, Ernst A, Bruno C, Cerf C, Chiron A, Souchay C, Burguet A, Jimenez C et al. Does Embryo Culture Medium Influence the Health and Development of Children Born after In Vitro Fertilization? PLoS One 2016;11: e0150857

Bowman P, McLaren A. Viability and growth of mouse embryos after in vitro culture and fusion. Journal of embryology and experimental morphology 1970;23: 693-704

Brandes RP. Endothelial dysfunction and hypertension. Hypertension 2014;64: 924-928.

Calle A, Fernandez-Gonzalez R, Ramos-Ibeas P, Laguna-Barraza R, Perez-Cerezales S, Bermejo-Alvarez P, Ramirez MA, Gutierrez-Adan A. Long-term and transgenerational effects of in vitro culture on mouse embryos. Theriogenology 2012;77: 785-793.

Carling SJ, Demment MM, Kjolhede CL, Olson CM. Breastfeeding duration and weight gain trajectory in infancy. Pediatrics 2015;135: 111-119. 
Carrasco B, Boada M, Rodríguez I, Coroleu B, Barri PN, Veiga A. Does culture medium influence offspring birth weight? Fertil Steril 2013;100: 1283-1288.

Ceelen M, van Weissenbruch MM, Prein J, Smit J, Vermeiden JP, Spreeuwenberg M, van Leeuwen FE, Delemarre-van de Waal HA. Growth during infancy and early childhood in relation to blood pressure and body fat measures at age 8-18 years of IVF children and spontaneously conceived controls born to subfertile parents. Hum Reprod 2009;24: 2788-2795.

Ceelen M, van Weissenbruch MM, Roos JC, Vermeiden JP, van Leeuwen FE, Delemarre-van de Waal HA. Body composition in children and adolescents born after in vitro fertilization or spontaneous conception. J Clin Endocrinol Metab 2007;92: 3417-3423.

Ceelen M, van Weissenbruch MM, Vermeiden JP, van Leeuwen FE, Delemarre-van de Waal HA. Cardiometabolic differences in children born after in vitro fertilization: follow-up study. J Clin Endocrinol Metab 2008;93: 1682-1688.

Ceelen M, van Weissenbruch MM, Vermeiden JP, van Leeuwen FE, Delemarre-van de Waal HA. Growth and development of children born after in vitro fertilization. Fertil Steril 2008;90: 1662-1673.

Chen M, Wu L, Zhao J, Wu F, Davies MJ, Wittert GA, Norman RJ, Robker RL, Heilbronn LK. Altered glucose metabolism in mouse and humans conceived by IVF. Diabetes 2014;63: 3189-3198.

Chiolero A, Kaufman JS, Paradis G. Why adjustment for current weight can bias the estimate of the effect of birth weight on blood pressure: shedding light using causal graphs. J Hypertens 2012;30: 1042-1045.

Chronopoulou E, Harper JC. IVF culture media: past, present and future. Hum Reprod Update 2015;21: 39-55.

Cole TJ, Lobstein T. Extended international (IOTF) body mass index cut-offs for thinness, overweight and obesity. Pediatr Obes 2012;7: 284-294.

Cracowski JL, Minson CT, Salvat-Melis M, Halliwill JR. Methodological issues in the assessment of skin microvascular endothelial function in humans. Trends Pharmacol Sci 2006;27: 503-508.

De Vos A, Janssens R, Van de Velde H, Haentjens P, Bonduelle M, Tournaye H, Verheyen G. The type of culture medium and the duration of in vitro culture do not influence birthweight of ART singletons. Hum Reprod 2015;30: 20-27.

Delle Piane L, Lin W, Liu X, Donjacour A, Minasi P, Revelli A, Maltepe E, Rinaudo PF. Effect of the method of conception and embryo transfer procedure on mid-gestation placenta and fetal development in an IVF mouse model. Human reproduction 2010;25: 2039-2046.

Delle Piane L, Lin W, Liu X, Donjacour A, Minasi P, Revelli A, Maltepe E, Rinaudo PF. Effect of the method of conception and embryo transfer procedure on mid-gestation placenta and fetal development in an IVF mouse model. Hum Reprod 2010;25: 2039-2046.

Doherty AS, Mann MRW, Tremblay KD, Bartolomei MS, Schultz RM. Differential effects of culture on imprinted H19 expression in the preimplantation mouse embryo. Biol Reprod 2000;62: 1526-1535.

Donjacour A, Liu X, Lin W, Simbulan R, Rinaudo PF. In vitro fertilization affects growth and glucose metabolism in a sex-specific manner in an outbred mouse model. Biol Reprod 2014;90: 80.

Draper ES, KurinczukJ, Abrams KR, Clarke M. Assessment of separate contributions to perinatal mortality of infertility history and treatment: a case-control analysis. Lancet 1999;353: 1746-1749.

Dumoulin JC, Land JA, Van Montfoort AP, Nelissen EC, Coonen E, Derhaag JG, Schreurs IL, Dunselman GA, Kester $A D$, Geraedts JP et al. Effect of in vitro culture of human embryos on birthweight of newborns. Hum Reprod 2010;25: 605-612.

Dyrlund TF, Kirkegaard K, Poulsen ET, Sanggaard KW, Hindkjaer JJ, Kjems J, Enghild J, Ingerslev HJ. Unconditioned commercial embryo culture media contain a large variety of non-declared proteins: a comprehensive proteomics analysis. Hum Reprod 2014;29: 2421-2430.

Eaton JL, Lieberman ES, Stearns C, Chinchilla M, Racowsky C. Embryo culture media and neonatal birthweight following IVF. Hum Reprod 2012;27: 375-379. 
Ecker DJ, Stein P, Xu Z, Williams CJ, Kopf GS, Bilker WB, Abel T, Schultz RM. Long-term effects of culture of preimplantation mouse embryos on behavior. Proceedings of the National Academy of Sciences of the United States of America 2004;101: 1595-1600.

Eckert J], Porter R, Watkins AJ, Burt E, Brooks S, Leese HJ, Humpherson PG, Cameron IT, Fleming TP. Metabolic induction and early responses of mouse blastocyst developmental programming following maternal low protein diet affecting life-long health. PLoS One 2012;7: e52791.

Eriksson J, Forsén T, Tuomilehto J, Osmond C, Barker D. Size at birth, childhood growth and obesity in adult life. Int J Obes Relat Metab Disord 2001;25: 735-740.

Eriksson JG, Forsén T, Tuomilehto J, Osmond C, Barker DJ. Early adiposity rebound in childhood and risk of Type 2 diabetes in adult life. Diabetologia 2003;46: 190-194.

Eskild A, Monkerud L, Tanbo T. Birthweight and placental weight; do changes in culture media used for IVF matter? Comparisons with spontaneous pregnancies in the corresponding time periods. Hum Reprod 2013;28: 32073214.

Evers JL. Female subfertility. Lancet 2002;360: 151-159.

Fabricius-Bjerre S, Jensen RB, Faerch K, Larsen T, Molgaard C, Michaelsen KF, Vaag A, Greisen G. Impact of birth weight and early infant weight gain on insulin resistance and associated cardiovascular risk factors in adolescence. PLoS One 2011;6: e20595.

Faddy MJ, Gosden MD, Gosden RG. A demographic projection of the contribution of assisted reproductive technologies to world population growth. Reprod Biomed Online 2018;36: 455-458.

Feelisch M. The use of nitric oxide donors in pharmacological studies. Naunyn Schmiedebergs Arch Pharmacol 1998;358: 113-122.

Fernández-Gonzalez R, Moreira P, Bilbao A, Jiménez A, Pérez-Crespo M, Ramírez MA, Rodríguez De Fonseca F, Pintado B, Gutiérrez-Adán A. Long-term effect of in vitro culture of mouse embryos with serum on mRNA expression of imprinting genes, development, and behavior. Proc Natl Acad Sci U S A 2004;101: 5880-5885.

Feuer S, Rinaudo P. Preimplantation stress and development. Birth Defects Res C Embryo Today 2012;96: 299-314.

Feuer S, Rinaudo P. From Embryos to Adults: A DOHaD Perspective on In Vitro Fertilization and Other Assisted Reproductive Technologies. Healthcare (Basel) 2016;4

Feuer SK, Liu X, Donjacour A, Lin W, Simbulan RK, Giritharan G, Piane LD, Kolahi K, Ameri K, Maltepe E et al. Use of a Mouse in Vitro Fertilization Model to Understand the Developmental Origins of Health and Disease Hypothesis. Endocrinology 2014: en20132081.

Feuer SK, Liu X, Donjacour A, Lin W, Simbulan RK, Giritharan G, Piane LD, Kolahi K, Ameri K, Maltepe E et al. Use of a mouse in vitro fertilization model to understand the developmental origins of health and disease hypothesis. Endocrinology 2014;155: 1956-1969.

Fleming TP, Kwong WY, Porter R, Ursell E, Fesenko I, Wilkins A, Miller DJ, Watkins AJ, Eckert JJ. The embryo and its future. Biol Reprod 2004; 71: 1046-1054.

Fleming TP, Velazquez MA, Eckert JJ. Embryos, DOHaD and David Barker. J Dev Orig Health Dis 2015;6: 377-383.

Fleming TP, Watkins AJ, Velazquez MA, Mathers JC, Prentice AM, Stephenson J, Barker M, Saffery R, Yajnik CS, Eckert Jj et al. Origins of lifetime health around the time of conception: causes and consequences. Lancet 2018.

Gardner DK, Hamilton R, McCallie B, Schoolcraft WB, Katz-Jaffe MG. Human and mouse embryonic development, metabolism and gene expression are altered by an ammonium gradient in vitro. Reproduction 2013;146: 49-61.

Gardner DK, Kelley RL. Impact of the IVF laboratory environment on human preimplantation embryo phenotype. J Dev Orig Health Dis 2017;8: 418-435.

Gardner DK, Lane M. Culture and selection of viable blastocysts: a feasible proposition for human IVF? Hum Reprod Update 1997;3: 367-382.

Gardner DK, Leese HJ. Concentrations of nutrients in mouse oviduct fluid and their effects on embryo development and metabolism in vitro. J Reprod Fertil 1990;88: 361-368. 
Gibbons WE, Cedars M, Ness RB, Society for Assisted Reproductive Technologies Writing G. Toward understanding obstetrical outcome in advanced assisted reproduction: varying sperm, oocyte, and uterine source and diagnosis. Fertility and sterility 2011;95: 1645-1649 e1641.

Gillman MW, Barker D, Bier D, Cagampang F, Challis J, Fall C, Godfrey K, Gluckman P, Hanson M, Kuh D et al. Meeting report on the 3rd International Congress on Developmental Origins of Health and Disease (DOHaD). Pediatric research 2007;61: 625-629.

Giritharan G, Talbi S, Donjacour A, Di Sebastiano F, Dobson AT, Rinaudo PF. Effect of in vitro fertilization on gene expression and development of mouse preimplantation embryos. Reproduction 2007;134: 63-72.

Gluckman PD, Hanson MA, Bateson P, Beedle AS, Law CM, Bhutta ZA, Anokhin KV, Bougneres P, Chandak GR, Dasgupta $\mathrm{P}$ et al. Towards a new developmental synthesis: adaptive developmental plasticity and human disease. Lancet 2009;373: 1654-1657.

Godfrey KM, Gluckman PD, Hanson MA. Developmental origins of metabolic disease: life course and intergenerational perspectives. Trends in endocrinology and metabolism: TEM 2010;21: 199-205.

Green MP, Mouat F, Miles HL, Hopkins SA, Derraik JG, Hofman PL, Peek JC, Cutfield WS. Phenotypic differences in children conceived from fresh and thawed embryos in in vitro fertilization compared with naturally conceived children. Fertil Steril 2013;99: 1898-1904.

Griesinger G, Kolibianakis EM, Diedrich K, Ludwig M. Ovarian stimulation for IVF has no quantitative association with birthweight: a registry study. Human reproduction 2008;23: 2549-2554.

Gruber I, Klein M. Embryo culture media for human IVF: which possibilities exist? J Turk Ger Gynecol Assoc 2011;12: 110-117.

Gu F, Deng M, Gao J, Wang Z, Ding C, Xu Y, Zhou C. The effects of embryo culture media on the birthweight of singletons via fresh or frozen-thawed embryo transfer: a large-scale retrospective study. BMC Pregnancy Childbirth 2016;16: 270.

Guo XY, Liu XM, Jin L, Wang T, Ullah K, Sheng JZ, Huang HF. Cardiovascular and metabolic profiles of offspring conceived by assisted reproductive technologies: a systematic review and meta-analysis. Fertil Steril 2017;107: 622-631 e625.

Han HD, Kiessling AA. In vivo development of transferred mouse embryos conceived in vitro in simple and complex media. Fertility and sterility 1988;50: 159-163.

Hart R, Norman RJ. The longer-term health outcomes for children born as a result of IVF treatment: Part I--General health outcomes. Hum Reprod Update 2013;19: 232-243.

Hart R, Norman RJ. The longer-term health outcomes for children born as a result of IVF treatment. Part II--Mental health and development outcomes. Hum Reprod Update 2013;19: 244-250.

Hassani F, Eftekhari-Yazdi P, Karimian L, Rezazadeh Valojerdi M, Movaghar B, Fazel M, Fouladi HR, Shabani F, Johansson L. The Effects of ISM1 Medium on Embryo Quality and Outcomes of IVF/ICSI Cycles. International journal of fertility \& sterility 2013; 7: 108-115.

Helmerhorst FM, Perquin DA, Donker D, Keirse MJ. Perinatal outcome of singletons and twins after assisted conception: a systematic review of controlled studies. Bmj 2004;328: 261.

Henningsen AK, Pinborg A. Birth and perinatal outcomes and complications for babies conceived following ART. Semin Fetal Neonatal Med 2014;19: 234-238.

Henningsen AK, Pinborg A, Lidegaard O, Vestergaard C, Forman JL, Andersen AN. Perinatal outcome of singleton siblings born after assisted reproductive technology and spontaneous conception: Danish national sibling-cohort study. Fertil Steril 2011;95: 959-963.

Hentemann M, Bertheussen K. New media for culture to blastocyst. Fertil Steril 2009;91: 878-883.

Hokken-Koelega AC, De Ridder MA, Lemmen RJ, Den Hartog H, De Muinck Keizer-Schrama SM, Drop SL. Children born small for gestational age: do they catch up? Pediatr Res 1995;38: 267-271. 
Imudia AN, Awonuga AO, Kaimal AJ, Wright DL, Styer AK, Toth TL. Elective cryopreservation of all embryos with subsequent cryothaw embryo transfer in patients at risk for ovarian hyperstimulation syndrome reduces the risk of adverse obstetric outcomes: a preliminary study. Fertility and sterility 2013;99: 168-173.

Jackson RA, Gibson KA, Wu YW, Croughan MS. Perinatal outcomes in singletons following in vitro fertilization: a meta-analysis. Obstet Gynecol 2004;103: 551-563.

Johnson TS, Engstrom JL, Warda JA, Kabat M, Peters B. Reliability of length measurements in full-term neonates. J Obstet Gynecol Neonatal Nurs 1998;27: 270-276.

Kagura J, Adair LS, Munthali RJ, Pettifor JM, Norris SA. Association Between Early Life Growth and Blood Pressure Trajectories in Black South African Children. Hypertension 2016;68: 1123-1131.

Khosla S, Dean W, Reik W, Feil R. Culture of preimplantation embryos and its long-term effects on gene expression and phenotype. Human reproduction update 2001;7: 419-427.

Khuc K, Blanco E, Burrows R, Reyes M, Castillo M, Lozoff B, Gahagan S. Adolescent metabolic syndrome risk is increased with higher infancy weight gain and decreased with longer breast feeding. Int J Pediatr 2012;2012: 478610

Kleijkers SH, Mantikou E, Slappendel E, Consten D, van Echten-Arends J, Wetzels AM, van Wely M, Smits LJ, van Montfoort AP, Repping S et al. Influence of embryo culture medium (G5 and HTF) on pregnancy and perinatal outcome after IVF: a multicenter RCT. Hum Reprod 2016;31: 2219-2230.

Kleijkers SH, van Montfoort AP, Bekers O, Coonen E, Derhaag JG, Evers JL, Dumoulin JC. Ammonium accumulation in commercially available embryo culture media and protein supplements during storage at 2-8 degrees C and during incubation at 37 degrees C. Hum Reprod 2016;31: 1192-1199.

Kleijkers SH, van Montfoort AP, Smits LJ, Coonen E, Derhaag JG, Evers JL, Dumoulin JC. Age of G-1 PLUS v5 embryo culture medium is inversely associated with birthweight of the newborn. Hum Reprod 2015;30: 1352-1357.

Kleijkers SH, van Montfoort AP, Smits LJ, Viechtbauer W, Roseboom TJ, Nelissen EC, Coonen E, Derhaag JG, Bastings L, Schreurs IE et al. IVF culture medium affects post-natal weight in humans during the first 2 years of life. Hum Reprod 2014;29: 661-669.

Koivurova S, Hartikainen AL, Sovio U, Gissler M, Hemminki E, Jarvelin MR. Growth, psychomotor development and morbidity up to 3 years of age in children born after IVF. Hum Reprod 2003;18: 2328-2336.

Krishnaveni GV, Veena SR, Wills AK, Hill JC, Karat SC, Fall CH. Adiposity, insulin resistance and cardiovascular risk factors in 9-10-year-old Indian children: relationships with birth size and postnatal growth. J Dev Orig Health Dis 2010;1: 403-411.

Kuiper D, Hoek A, la Bastide-van Gemert S, Seggers J, Mulder DJ, Haadsma M, Heineman MJ, Hadders-Algra M. Cardiovascular health of 9-year-old IVF offspring: no association with ovarian hyperstimulation and the in vitro procedure. Hum Reprod 2017;32: 2540-2548.

Lane M, Gardner DK. Ammonium induces aberrant blastocyst differentiation, metabolism, pH regulation, gene expression and subsequently alters fetal development in the mouse. Biol Reprod 2003;69: 1109-1117.

Lawitts JA, Biggers JD. Optimization of mouse embryo culture media using simplex methods. J Reprod Fertil 1991;91: 543-556.

Lee SH, Lee MY, Chiang TL, Lee MS, Lee MC. Child growth from birth to 18 months old born after assisted reproductive technology--results of a national birth cohort study. Int J Nurs Stud 2010;47: 1159-1166.

Lei X, Chen Y, Ye J, Ouyang F, Jiang F, Zhang J. The optimal postnatal growth trajectory for term small for gestational age babies: a prospective cohort study. J Pediatr 2015;166: 54-58.

Lemmen JG, Pinborg A, Rasmussen S, Ziebe S. Birthweight distribution in ART singletons resulting from embryo culture in two different culture media compared with the national population. Human reproduction 2014;29: 2326-2332.

Leunissen RW, Kerkhof GF, Stijnen T, Hokken-Koelega A. Timing and tempo of first-year rapid growth in relation to cardiovascular and metabolic risk profile in early adulthood. JAMA 2009;301: 2234-2242. 
Leunissen RW, Stijnen T, Hokken-Koelega AC. Influence of birth size on body composition in early adulthood: the programming factors for growth and metabolism (PROGRAM)-study. Clin Endocrinol (Oxf) 2009;70: 245-251.

Lim D, Bowdin SC, Tee L, Kirby GA, Blair E, Fryer A, Lam W, Oley C, Cole T, Brueton LA et al. Clinical and molecular genetic features of Beckwith-Wiedemann syndrome associated with assisted reproductive technologies. Human reproduction 2009;24: 741-747.

Lin S, Li M, Lian Y, Chen L, Liu P. No effect of embryo culture media on birthweight and length of newborns. Hum Reprod 2013;28: 1762-1767.

Lv PP, Meng Y, Lv M, Feng C, Liu Y, Li JY, Yu DQ, Shen Y, Hu XL, Gao Q et al. Altered thyroid hormone profile in offspring after exposure to high estradiol environment during the first trimester of pregnancy: a cross-sectional study. BMC Med 2014;12: 240.

MacLaughlin SM, Walker SK, Roberts CT, Kleemann DO, McMillen IC. Periconceptional nutrition and the relationship between maternal body weight changes in the periconceptional period and feto-placental growth in the sheep. J Physiol 2005;565: 111-124.

Makinen S, Soderstrom-Anttila V, Vainio J, Suikkari AM, Tuuri T. Does long in vitro culture promote large for gestational age babies? Hum Reprod 2013;28: 828-834.

Malchau SS, Loft A, Larsen EC, Aaris Henningsen AK, Rasmussen S, Andersen AN, Pinborg A. Perinatal outcomes in 375 children born after oocyte donation: a Danish national cohort study. Fertility and sterility 2013;99: $1637-1643$.

Marino JL, Moore VM, Willson KJ, Rumbold A, Whitrow MJ, Giles LC, Davies MJ. Perinatal outcomes by mode of assisted conception and sub-fertility in an Australian data linkage cohort. PloS one 2014;9: e80398.

Market-Velker BA, Fernandes AD, Mann MR. Side-by-side comparison of five commercial media systems in a mouse model: suboptimal in vitro culture interferes with imprint maintenance. Biol Reprod 2010;83: 938-950.

McDonald SD, Han Z, Mulla S, Murphy KE, Beyene J, Ohlsson A, Knowledge Synthesis G. Preterm birth and low birth weight among in vitro fertilization singletons: a systematic review and meta-analyses. Eur J Obstet Gynecol Reprod Biol 2009;146: 138-148.

Messerlian C, Maclagan L, Basso O. Infertility and the risk of adverse pregnancy outcomes: a systematic review and meta-analysis. Human reproduction 2013;28: 125-137.

Meyer JS, Novak MA. Minireview: Hair cortisol: a novel biomarker of hypothalamic-pituitary-adrenocortical activity. Endocrinology 2012;153: 4120-4127.

Morbeck DE, Krisher RL, Herrick JR, Baumann NA, Matern D, Moyer T. Composition of commercial media used for human embryo culture. Fertil Steril 2014;102: 759-766 e759.

Morbeck DE, Paczkowski M, Fredrickson JR, Krisher RL, Hoff HS, Baumann NA, Moyer T, Matern D. Composition of protein supplements used for human embryo culture. J Assist Reprod Genet 2014;31: 1703-1711.

Nakashima A, Araki R, Tani H, Ishihara O, Kuwahara A, Irahara M, Yoshimura Y, Kuramoto T, Saito H, Nakaza A et al. Implications of assisted reproductive technologies on term singleton birth weight: an analysis of 25,777 children in the national assisted reproduction registry of Japan. Fertility and sterility 2013;99: 450-455.

National. High Blood Pressure Education Program Working Group on High Blood Pressure in Children and Adolescents. The fourth report on the diagnosis, evaluation, and treatment of high blood pressure in children and adolescents. Pediatrics 2004;114: 555-576.

Nelissen EC, Van Montfoort AP, Coonen E, Derhaag JG, Geraedts JP, Smits LJ, Land JA, Evers JL, Dumoulin JC. Further evidence that culture media affect perinatal outcome: findings after transfer of fresh and cryopreserved embryos. Human reproduction 2012;27: 1966-1976.

Nelissen EC, Van Montfoort AP, Smits LJ, Menheere PP, Evers JL, Coonen E, Derhaag JG, Peeters LL, Coumans AB, Dumoulin JC. IVF culture medium affects human intrauterine growth as early as the second trimester of pregnancy. Hum Reprod 2013;28: 2067-2074. 
Onal H, Ercan O, Adal E, Ersen A, Onal Z. Subclinical hypothyroidism in in vitro fertilization babies. Acta Paediatr 2012;101: e248-252.

Palermo G, Joris H, Devroey P, Van Steirteghem AC. Pregnancies after intracytoplasmic injection of single spermatozoon into an oocyte. Lancet 1992;340: 17-18.

Pandey S, Shetty A, Hamilton M, Bhattacharya S, Maheshwari A. Obstetric and perinatal outcomes in singleton pregnancies resulting from IVF/ICSI: a systematic review and meta-analysis. Hum Reprod Update 2012;18: 485-503.

Patro-Golab B, Zalewski BM, Kouwenhoven SM, Karas J, Koletzko B, Bernard van Goudoever J, Szajewska H. Protein Concentration in Milk Formula, Growth, and Later Risk of Obesity: A Systematic Review. J Nutr 2016;146: 551-564.

Pelinck MJ, Hadders-Algra M, Haadsma ML, Nijhuis WL, Kiewiet SM, Hoek A, Heineman MJ, Middleburg KJ. Is the birthweight of singletons born after IVF reduced by ovarian stimulation or by IVF laboratory procedures? Reproductive biomedicine online 2010;21: 245-251.

Pinborg A, Henningsen AA, Loft A, Malchau SS, Forman J, Andersen AN. Large baby syndrome in singletons born after frozen embryo transfer (FET): is it due to maternal factors or the cryotechnique? Hum Reprod 2014;29: 618-627.

Pinborg A, Wennerholm UB, Romundstad LB, Loft A, Aittomaki K, Söderström-Anttila V, Nygren KG, Hazekamp J, Bergh C. Why do singletons conceived after assisted reproduction technology have adverse perinatal outcome? Systematic review and meta-analysis. Hum Reprod Update 2013;19: 87-104.

Pontesilli M, Painter RC, Grooten IJ, van der Post JA, Mol BW, Vrijkotte TG, Repping S, Roseboom TJ. Subfertility and assisted reproduction techniques are associated with poorer cardiometabolic profiles in childhood. Reprod Biomed Online 2015;30: 258-267.

Pourteymour Fard Tabrizi F, Alipoor B, Mehrzad Sadaghiani M, Ostadrahimi A, Malek Mahdavi A. Metabolic Syndrome and Its Characteristics among Reproductive-Aged Women with Polycystic Ovary Syndrome: A Cross-sectional Study in Northwest Iran. Int J Fertil Steril 2013;6: 244-249.

Puissant C, Abraham P, Durand S, Humeau-Heurtier A, Faure S, Leftheriotis G, Rousseau P, Mahe G. Reproducibility of non-invasive assessment of skin endothelial function using laser Doppler flowmetry and laser speckle contrast imaging. PLoS One 2013;8: e61320.

Rexhaj E, Paoloni-Giacobino A, Rimoldi SF, Fuster DG, Anderegg M, Somm E, Bouillet E, Allemann Y, Sartori C, Scherrer $\cup$. Mice generated by in vitro fertilization exhibit vascular dysfunction and shortened life span. J Clin Invest 2013;123: 5052-5060.

Rimoldi SF, Sartori C, Rexhaj E, Bailey DM, de Marchi SF, McEneny J, Arx R, Cerny D, Duplain H, Germond M et al. Antioxidants improve vascular function in children conceived by assisted reproductive technologies: A randomized double-blind placebo-controlled trial. Eur J Prev Cardiol 2015;22: 1399-1407.

Rimoldi SF, Sartori C, Rexhaj E, Cerny D, Von Arx R, Soria R, Germond M, Allemann Y, Scherrer U. Vascular dysfunction in children conceived by assisted reproductive technologies: underlying mechanisms and future implications. Swiss Med Wkly 2014;144: w13973.

Roberts SA, Vail A. On the appropriate interpretation of evidence: the example of culture media and birth weight. Hum Reprod 2017;32: 1151-1154.

Romundstad LB, Romundstad PR, Sunde A, von During V, Skjaerven R, Gunnell D, Vatten LJ. Effects of technology or maternal factors on perinatal outcome after assisted fertilisation: a population-based cohort study. Lancet 2008;372: 737-743.

Rooke JA, McEvoy TG, Ashworth CJ, Robinson JJ, Wilmut I, Young LE, Sinclair KD. Ovine fetal development is more sensitive to perturbation by the presence of serum in embryo culture before rather than after compaction. Theriogenology 2007;67: 639-647.

Roseboom T, de Rooij S, Painter R. The Dutch famine and its long-term consequences for adult health. Early Hum Dev 2006;82: 485-491. 
Roseboom TJ, Painter RC, van Abeelen AF, Veenendaal MV, de Rooij SR. Hungry in the womb: what are the consequences? Lessons from the Dutch famine. Maturitas 2011;70: 141-145.

Roseboom TJ, van der Meulen JH, van Montfrans GA, Ravelli AC, Osmond C, Barker DJ, Bleker OP. Maternal nutrition during gestation and blood pressure in later life. J Hypertens 2001;19: 29-34.

Rumball CW, Bloomfield FH, Oliver MH, Harding JE. Different periods of periconceptional undernutrition have different effects on growth, metabolic and endocrine status in fetal sheep. Pediatr Res 2009;66: 605-613.

Rumbold AR, Moore VM, Whitrow MJ, Oswald TK, Moran LJ, Fernandez RC, Barnhart KT, Davies MJ. The impact of specific fertility treatments on cognitive development in childhood and adolescence: a systematic review. Hum Reprod 2017;32: 1489-1507.

Sakka SD, Loutradis D, Kanaka-Gantenbein C, Margeli A, Papastamataki M, Papassotiriou I, Chrousos GP. Absence of insulin resistance and low-grade inflammation despite early metabolic syndrome manifestations in children born after in vitro fertilization. Fertil Steril 2010;94: 1693-1699.

Sauvé B, Koren G, Walsh G, Tokmakejian S, Van Uum SH. Measurement of cortisol in human hair as a biomarker of systemic exposure. Clin Invest Med 2007;30: E183-191.

Savage JS, Birch LL, Marini M, Anzman-Frasca S, Paul IM. Effect of the INSIGHT Responsive Parenting Intervention on Rapid Infant Weight Gain and Overweight Status at Age 1 Year: A Randomized Clinical Trial. JAMA Pediatr 2016;170: 742-749.

Sazonova A, Kallen K, Thurin-Kjellberg A, Wennerholm UB, Bergh C. Obstetric outcome in singletons after in vitro fertilization with cryopreserved/thawed embryos. Human reproduction 2012;27: 1343-1350.

Schellong K, Schulz S, Harder T, Plagemann A. Birth weight and long-term overweight risk: systematic review and a meta-analysis including 643,902 persons from 66 studies and 26 countries globally. PLoS One 2012;7: e47776.

Schendelaar P, La Bastide-Van Gemert S, Heineman MJ, Middelburg KJ, Seggers J, Van den Heuvel ER, Hadders-Algra M. Subfertility factors rather than assisted conception factors affect cognitive and behavioural development of 4-year-old singletons. Reprod Biomed Online 2016;33: 752-762.

Scherrer U, Rimoldi SF, Rexhaj E, Stuber T, Duplain H, Garcin S, de Marchi SF, Nicod P, Germond M, Allemann Y et al. Systemic and pulmonary vascular dysfunction in children conceived by assisted reproductive technologies. Circulation 2012;125: 1890-1896.

Schieve LA, Meikle SF, Ferre C, Peterson HB, Jeng G, Wilcox LS. Low and very low birth weight in infants conceived with use of assisted reproductive technology. The New England journal of medicine 2002;346: 731-737.

Schonbeck Y, Talma H, van Dommelen P, Bakker B, Buitendijk SE, HiraSing RA, van Buuren S. The world's tallest nation has stopped growing taller: the height of Dutch children from 1955 to 2009. Pediatr Res 2013;73: 371-377.

Schönbeck Y, Talma H, van Dommelen P, Bakker B, Buitendijk SE, Hirasing RA, van Buuren S. Increase in prevalence of overweight in Dutch children and adolescents: a comparison of nationwide growth studies in 1980, 1997 and 2009. PLoS One 2011;6: e27608.

Schwarzer C, Esteves TC, Arauzo-Bravo MJ, Le Gac S, Nordhoff V, Schlatt S, Boiani M. ART culture conditions change the probability of mouse embryo gestation through defined cellular and molecular responses. Human reproduction 2012;27: 2627-2640.

Scott KA, Yamazaki Y, Yamamoto M, Lin Y, Melhorn SJ, Krause EG, Woods SC, Yanagimachi R, Sakai RR, Tamashiro KL. Glucose parameters are altered in mouse offspring produced by assisted reproductive technologies and somatic cell nuclear transfer. Biol Reprod 2010;83: 220-227.

Sinclair KD, McEvoy TG, Carolan C, Maxfield EK, Maltin CA, Young LE, Wilmut I, Robinson Jل, Broadbent PJ. Conceptus growth and development following in vitro culture of ovine embryos in media supplemented with bovine sera. Theriogenology 1998;49: 218-218.

Singhal A. Long-Term Adverse Effects of Early Growth Acceleration or Catch-Up Growth. Ann Nutr Metab 2017;70: 236-240. 


\section{Chapter 6}

Singhal A, Cole TJ, Fewtrell M, Deanfield J, Lucas A. Is slower early growth beneficial for long-term cardiovascular health? Circulation 2004;109: 1108-1113.

Singhal A, Lucas A. Early origins of cardiovascular disease: is there a unifying hypothesis? Lancet 2004;363: 16421645.

Skovlund E, Fenstad GU. Should we always choose a nonparametric test when comparing two apparently nonnormal distributions? J Clin Epidemio/ 2001;54: 86-92.

Stalder T, Kirschbaum C, Alexander N, Bornstein SR, Gao W, Miller R, Stark S, Bosch JA, Fischer JE. Cortisol in hair and the metabolic syndrome. J Clin Endocrinol Metab 2013;98: 2573-2580.

Steptoe PC, Edwards RG. Birth after the reimplantation of a human embryo. Lancet 1978;2: 366.

Summers MC, Biggers JD. Chemically defined media and the culture of mammalian preimplantation embryos: historical perspective and current issues. Hum Reprod Update 2003;9: 557-582.

Sunde A, Brison D, Dumoulin J, Harper J, Lundin K, Magli MC, Van den Abbeel E, Veiga A. Time to take human embryo culture seriously. Hum Reprod 2016;31: 2174-2182.

Taine M, Stengel B, Forhan A, Carles S, Botton J, Charles MA, Heude B, Group EM-CCS. Rapid Early Growth May Modulate the Association Between Birth Weight and Blood Pressure at 5 Years in the EDEN Cohort Study. Hypertension 2016;68: 859-865.

Thompson JG, Gardner DK, Pugh PA, McMillan WH, Tervit HR. Lamb birth weight is affected by culture system utilized during in vitro pre-elongation development of ovine embryos. Biol Reprod 1995;53: 1385-1391.

Thouas GA, Jones GM, Trounson AO. The 'GO' system--a novel method of microculture for in vitro development of mouse zygotes to the blastocyst stage. Reproduction 2003;126: 161-169.

Touwslager RN, Gerver WJ, Tan FE, Gielen M, Zeegers MP, Zimmermann LJ, Houben AJ, Blanco CE, Stehouwer CD, Mulder AL. Influence of growth during infancy on endothelium-dependent vasodilatation at the age of 6 months. Hypertension 2012;60: 1294-1300

Touwslager RN, Houben AJ, Gielen M, Zeegers MP, Stehouwer CD, Zimmermann LJ, Kessels AG, Gerver WJ, Blanco CE, Mulder AL. Endothelial vasodilatation in newborns is related to body size and maternal hypertension. $J$ Hypertens 2012;30: 124-131.

Touwslager RN, Houben AJ, Tan FE, Gielen M, Zeegers MP, Stehouwer CD, Gerver WJ, Westerterp KR, Wouters L, Blanco CE et al. Growth and endothelial function in the first 2 years of life. J Pediatr 2015;166: 666-671 e661.

Turner J, Belch J, Khan F. Current concepts in assessment of microvascular endothelial function using laser Doppler imaging and iontophoresis. Trends Cardiovasc Med 2008;18: 109-116.

Valenzuela-Alcaraz B, Crispi F, Bijnens B, Cruz-Lemini M, Creus M, Sitges M, Bartrons J, Civico S, Balasch J, Gratacos E. Assisted reproductive technologies are associated with cardiovascular remodeling in utero that persists postnatally. Circulation 2013;128: 1442-1450

Veena SR, Hegde BG, Ramachandraiah S, Krishnaveni GV, Fall CH, Srinivasan K. Relationship between adiposity and cognitive performance in 9-10-year-old children in South India. Arch Dis Child 2014;99: 126-134.

Velazquez MA, Sheth B, Smith SJ, Eckert JJ, Osmond C, Fleming TP. Insulin and branched-chain amino acid depletion during mouse preimplantation embryo culture programmes body weight gain and raised blood pressure during early postnatal life. Biochim Biophys Acta 2017;1864: 590-600.

Vergouw CG, Kostelijk EH, Doejaaren E, Hompes PG, Lambalk CB, Schats R. The influence of the type of embryo culture medium on neonatal birthweight after single embryo transfer in IVF. Hum Reprod 2012;27: 2619-2626.

Vermeiden JP, Bernardus RE. Are imprinting disorders more prevalent after human in vitro fertilization or intracytoplasmic sperm injection? Fertil Steril 2013;99: 642-651.

Vidal M, Vellve K, Gonzalez-Comadran M, Robles A, Prat M, Torne M, Carreras R, Checa MA. Perinatal outcomes in children born after fresh or frozen embryo transfer: a Catalan cohort study based on 14,262 newborns. Fertil Steril 2017;107: 940-947. 
Visser GH, Eilers PH, Elferink-Stinkens PM, Merkus HM, Wit JM. New Dutch reference curves for birthweight by gestational age. Early Hum Dev 2009;85: 737-744.

Vrooman LA, Bartolomei MS. Can assisted reproductive technologies cause adult-onset disease? Evidence from human and mouse. Reprod Toxicol 2017;68: 72-84.

Watkins AJ, Lucas ES, Wilkins A, Cagampang FR, Fleming TP. Maternal periconceptional and gestational low protein diet affects mouse offspring growth, cardiovascular and adipose phenotype at 1 year of age. PLoS One 2011;6: e28745.

Watkins AJ, Platt D, Papenbrock T, Wilkins A, Eckert J, Kwong WY, Osmond C, Hanson M, Fleming TP. Mouse embryo culture induces changes in postnatal phenotype including raised systolic blood pressure. Proc Natl Acad Sci U S A 2007;104: 5449-5454.

Wennerholm UB, Henningsen AK, Romundstad LB, Bergh C, Pinborg A, Skjaerven R, Forman J, Gissler M, Nygren KG, Tiitinen A. Perinatal outcomes of children born after frozen-thawed embryo transfer: a Nordic cohort study from the CONARTaS group. Human reproduction 2013;28: 2545-2553.

Woldringh GH, Hendriks JC, van Klingeren J, van Buuren S, Kollee LA, Zielhuis GA, Kremer JA. Weight of in vitro fertilization and intracytoplasmic sperm injection singletons in early childhood. Fertil Steril 2011;95: 2775-2777.

Woo JG. Fast, Slow, High, and Low: Infant and Childhood Growth as Predictors of Cardiometabolic Outcomes. J Pediatr 2017;186: 14-16.

Wunder D, Ballabeni P, Roth-Kleiner M, Primi MP, Senn A, Chanson A, Germond M, Leyvraz C. Effect of embryo culture media on birthweight and length in singleton term infants after IVF-ICSI. Swiss medical weekly 2014;144: w14038. http://www.openepi.com.

Yeung EH, Sundaram R, Bell EM, Druschel C, Kus C, Xie Y, Buck Louis GM. Infertility treatment and children's longitudinal growth between birth and 3 years of age. Hum Reprod 2016;31: 1621-1628,

Yin TL, Zhang Y, Li SJ, Zhao M, Ding JL, Xu WM, Yang J. Culture media influenced laboratory outcomes but not neonatal birth weight in assisted reproductive technology.J Huazhong Univ Sci Technolog Med Sci 2015;35: 932-937.

Young LE, Fernandes K, McEvoy TG, Butterwith SC, Gutierrez CG, Carolan C, Broadbent PJ, Robinson JJ, Wilmut I, Sinclair KD. Epigenetic change in IGF2R is associated with fetal overgrowth after sheep embryo culture. Nature genetics 2001;27: 153-154.

Zander DL, Thompson JG, Lane M. Perturbations in mouse embryo development and viability caused by ammonium are more severe after exposure at the cleavage stages. Biol Reprod 2006;74: 288-294.

Zandstra H, Van Montfoort AP, Dumoulin JC. Does the type of culture medium used influence birthweight of children born after IVF? Hum Reprod 2015;30: 2693.

Zegers-Hochschild F, Masoli D, Schwarze JE, laconelli A, Borges E, Pacheco IM. Reproductive performance in oocyte donors and their recipients: comparative analysis from implantation to birth and lactation. Fertility and sterility 2010;93: 2210-2215.

Zhan QT, Pan PP, Xu XR, Lou HY, Lou YY, Jin F. An overview of studies on psychological well-being in children born following assisted reproductive technologies. J Zhejiang Univ Sci B 2013;14: 947-960.

Zhu J, Li M, Chen L, Liu P, Qiao J. The protein source in embryo culture media influences birthweight: a comparative study between G1 v5 and G1-PLUS v5. Human reproduction 2014;29: 1387-1392.

Zhu J, Lin S, Li M, Chen L, Lian Y, Liu P, Qiao J. Effect of in vitro culture period on birthweight of singleton newborns. Hum Reprod 2014;29: 448-454.

Ziebe S, Loft A, Povlsen BB, Erb K, Agerholm I, Aasted M, Gabrielsen A, Hnida C, Zobel DP, Munding B et al. A randomized clinical trial to evaluate the effect of granulocyte-macrophage colony-stimulating factor (GM-CSF) in embryo culture medium for in vitro fertilization. Fertility and sterility 2013;99: 1600-1609. 


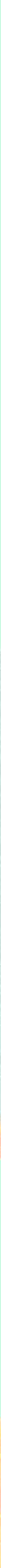




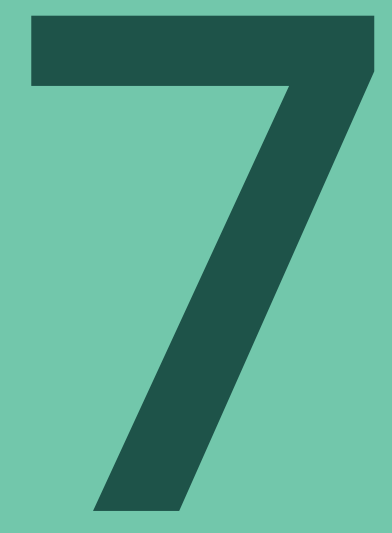

\section{General discussion and future prospects}





\section{GENERAL DISCUSSION AND FUTURE PROSPECTS}

Although an increased risk of an adverse perinatal outcome in IVF offspring has been shown in numerous studies, knowledge about the exact aetiology is still limited and multiple theories explaining the observed differences have been proposed. In this thesis we focused on the effects of culture medium on the health of the IVF offspring that can have possible long-lasting consequences.

\section{Effects of culture medium on perinatal outcome}

The existence of a possible association between culture medium and perinatal outcome in the human is still under debate and evidence is scarce. Therefore, we performed a structured literature review of the effects of culture medium on birthweight of the resulting IVF offspring in human and animals (Chapter 2). There was evidence in humans for a culture medium effect, but the evidence was of limited quantity and had methodological weaknesses related to the retrospective study designs of all studies. Also, hardly any trials compared the same culture media making a meta-analysis impossible. Since the publication of this review, an additional three retrospective studies and one large prospective multicenter randomized controlled trial (RCT) have been published. The retrospective studies all reported no difference in birthweight between their investigated culture media (De Vos et al., 2015, Gu et al., 2016, Yin et al., 2015). Yin and colleagues compared 673 singletons born as a result of embryo culture in either Quinn's Advantage medium (SAGE, CA, USA), Single Step Medium (SSM Irvine Scientific, USA) or Continuous Single Culture medium (CSC Irvine Scientific, USA) (Yin et al., 2015). Gu and colleagues compared 2370 singletons born after embryo culture in G5 (Vitrolife, Goteborg, Sweden) and Quinns Advantage (SAGE, CA, USA). This comparison was also performed by Lin et al (2013) who did not find an effect either. De Vos and colleagues compared the outcome of 2098 singletons that were born after embryo culture in either G3 or G5 culture medium (Vitrolife, Goteborg, Sweden) or Universal IVF Medium, EmbryoAssist and BlastAssist (Medicult, De Pinte, Belgium) (De Vos et al., 2015). Medicult is supplemented with synthetic serum replacement (De Vos et al., 2015). Vitrolife fertilization medium contains fructose, lactate, non-essential amino acids and EDTA, whereas Medicult fertilization medium does not. The cleavage medium of Medicult contains additional carbohydrates and different vitamins, where Vitrolife cleavage medium contains methionine, hyaluron and lipoic acid. Both blastocyst media are quite similar in their amino acid composition, except for arginine, which is present in Vitrolife but not in Medicult Blastocyst medium (De Vos et al., 2015, Eskild et al., 2013). Other studies comparing media with differences in the presence of essential amino acids did not show a difference in birthweight either (Eaton et al., 2012, Lin et al., 2013). Although the sample sizes of these three studies were large, they were all based on retrospective data, with their inherent limitations. 
In 2016, the results of our large prospective multicenter RCT comparing live birth rates and postnatal effects on the offspring of cycles with embryo culture in either G5 (Vitrolife, Goteborg, Sweden) or HTF (Lonza, Verviers, Belgium) showed that culture medium can have an effect on perinatal outcome (Kleijkers et al., 2016). A total of 300 live born singletons from 836 IVF cycles were compared. Although the difference in live birth rate was not significant (HTF 37.9\% vs G5 44.1\%, $P=0.08$ ), there was a clearly significant $(P=0.005)$ difference in birthweight between the two groups (mean difference 158gr 95\% Cl 42-275gr) (Kleijkers et al., 2016). One of the important differences is that the $\mathrm{G} 5$ medium contains specific amino acids, whereas the HTF medium completely lacks amino acids. Studies in human and animal embryos have shown that the embryo development in vitro can be improved by the addition of non-essential amino acids to the medium (Gardner and Lane, 1997, Summers and Biggers, 2003). Although the debate on culture media and their effect on perinatal outcome is still ongoing, this rigorously performed RCT, compared to all other, weaker designed, studies, provides robust evidence that culture medium can have an effect on perinatal outcome (Roberts and Vail, 2017).

Most studies comparing culture media outcome use a retrospective design comparing different culture media that were used in consecutive periods of time, which introduces bias since many other factors might have changed over time as well. Especially in IVF, numerous other factors such as stimulation protocols, oxygen concentration during embryo culture and number of embryos that are transferred can affect perinatal outcome, apart from the culture medium that was used (Gardner and Kelley, 2017). This strongly limits the value of using historical controls.

Although in human studies evidence is still limited, the association between culture medium and birthweight has been repeatedly confirmed in animal studies (Feuer et al., 2014, Velazquez et al., 2017). Also, effects on postnatal outcome by culture conditions or peri-implantation environment of the embryos have been repeatedly confirmed in animal studies (MacLaughlin et al., 2005, Rumball et al., 2009, Watkins et al., 2011, Watkins et al., 2007). In animal studies it has been observed that alterations in the culture medium can induce changes in long-term health parameters like glucose metabolism, leptin levels and blood pressure (Feuer and Rinaudo, 2012, Scott et al., 2010, Watkins et al., 2011). This knowledge implies that the embryo culture medium might be more influential on the future IVF offspring than we used to believe.

Long-term effects of culture medium

Regarding the still young age of human IVF progeny, it is not clear how the observed differences in birthweight of the IVF population relate to health in (late) adulthood. To gain more insight in effects of culture medium on long-term health of IVF offspring, we performed a 
prospective follow-up study that included all eligible singletons from our first study cohort, originating from IVF/ICSI treatments between 2003 and 2006 (Dumoulin et al., 2010). At birth, the children from the Vitrolife group were on average heavier than the children from the Cook group. We analysed anthropometrics, growth, body composition, cardiovascular development and cognitive development of children born after culture in one of two different culture media (G1 ${ }^{\text {TM }}$ Version 3 (Vitrolife group) or K-SICM (Cook group)) at 9 years of age. We showed that at 9 years of age the Vitrolife group was on average still heavier with a higher BMI and more subcutaneous truncal fat (Chapter 3). Cardiovascular development was similar for the two groups (Chapter 4). Children born after IVF have been described to exhibit more risk factors for cardiovascular disease, such as increased blood pressure and higher fasting glucose levels (Guo et al., 2017). It has also been reported that children born after IVF display endothelial dysfunction and vascular stiffness compared to controls (Scherrer et al., 2012). In our study we did not confirm these findings. Although we did not include a non-IVF control population, we compared the bloodpressure of the IVF children in our cohort with (non-IVF) reference values and found no differences. We also used a control cohort to compare the lipid profile and TSH values of the IVF cohort with a non-IVF cohort and found the lipid profile to be more favourable in the IVF children.

Although the cardiovascular results of our study are reassuring for now, the higher amount of adipose tissue and higher BMI in the Vitrolife group, might imply a future risk factor. Our results are in line with observations showing that higher birthweight will result in higher adult weight and more adiposity (Schellong et al., 2012), possibly contributing to a higher risk for cardiovascular disease in adult life.

Several studies suggest that the relation between lower BW and cardiovascular disease as observed by Barker (Barker, 2006), is more pronounced if the child displays an increased growth velocity during early postnatal life, i.e. so called catch-up growth (Eriksson et al., 2001, Hokken-Koelega et al., 1995, Khuc et al., 2012, Leunissen et al., 2009). Young adults born small for gestational age (SGA) that have shown rapid weigh gain between birth and adulthood had a higher risk for cardiovascular disease as compared to young adults without rapid weight gain (Leunissen et al., 2009, Singhal, 2017). A similar relation is seen in young adults with normal birthweight (Singhal, 2017). This suggests that it is not the BW per se that is related to CVD, but that growth velocity is even more important.

To analyse whether the relation with growth velocity was also present in our group, we analysed infant and childhood growth velocity in relation to cardiovascular outcome and obesity in our children at 9 years of age (Chapter 6). We showed that also in our cohort growth velocity was associated with endothelial dysfunction and both systolic and diastolic blood pressure. We reported clinically relevant effect sizes of 4-5.5 mmHg per one unit 
increase in weight z-score. There was however no difference in effect size between the two culture medium groups. Whether there is a difference in growth velocity between the two groups deserves to be further investigated.

We also analysed cognitive development in our prospective cohort study. The cognitive development was comparable between the two culture media groups at 9 years of age (Chapter 5). Only a limited amount of studies reporting on cognitive development in IVF children are available so far. While most studies show normal cognitive and behavioural development in IVF children (Berry et al., 2013, Schendelaar et al., 2016), negative effects on cognitive outcome of fertility treatments have also been reported (Rumbold et al., 2017). Only one previous study (Bouillon et al., 2016) compared the effect of different culture media on developmental domains including social and motor development, language comprehension and number knowledge. They found a significantly increased risk for developmental problems on all domains in children who were born after embryo culture in Single Step Medium (SSM, Irvine scientific) as compared to Global culture medium (LifeGlobal) (Bouillon et al., 2016).

\section{Culture media composition}

With regard to the culture media we used in our study (Cook and Vitrolife) there are several important differences in the ingredients used. The most important differences are the osmolality, the presence of hyaluron in the Vitrolife medium, glucose content, and different lactate and pyruvate content resulting in a higher lactate/pyruvate ratio in the Vitrolife medium as compared to the Cook medium. It has been shown that changing the lactate/ pyruvate ratio in the culture medium during the pronuclear stage can result in different post-natal growth rates in the mouse (Banrezes et al., 2011). Also the type of glutamin differed between the two culture media. The version of the Cook medium that was used in our study contained the free L-glutamin, which is known to decompose spontaneously, releasing ammonium. The Vitrolife medium however contained the more stable dipeptide alanyl-glutamine (Kleijkers et al., 2016). In 2015, our group showed that age of the G-1 PLUS v5 (Vitrolife AB, Goteborg, Sweden) culture medium was inversely associated with the birthweight of the newborns (Kleijkers et al., 2015). Also, we showed that ammonium accumulates during incubation and storage of the medium, which was attributable to unstable glutamin (even in the presence of the more stable form of glutamin) and the protein supplement that was used (Kleijkers et al., 2016).

Many different brands and types of culture media are available on the market, all with large differences in composition. More important than to focus on identifying superior "brands" for culture media, is to unravel the possible effects of specific ingredients on the embryo and the subsequent offspring. For example, it is known that amino acids such as 
methionine and vitamins taken up by the embryo can cause epigenetic changes (Gardner and Kelley, 2017). Also there is ongoing debate on the optimal concentration and composition of the protein source that is used in the culture medium (Morbeck et al., 2014). Historically, only human serum albumin (HSA) was used as a protein supplement, but nowadays also recombinant albumin or hyaluronic acid is used as a replacement for HSA (Morbeck et al., 2014). It is not yet clear whether the contaminants that are present in HSA can affect the embryo development in vitro, nor whether the recombinant albumin has the same (or possibly even better) positive effects on embryo culture as HSA without the possible negative effect of the contaminants (Dyrlund et al., 2014, Morbeck et al., 2014).

\section{Difficulties in culture medium research}

One of the most important limitations of research on culture medium effects in human IVF is the fact that the exact composition of the culture media is considered commercially confidential information. Most manufacturers do not disclose the exact composition of their culture media to clinicians, embryologists or researchers. The lack of knowledge about the exact composition deprives us from a chance to improve the culture media. To elucidate the relationship between embryo culture medium composition and birthweight and health outcomes, it is mandatory that this unjustifiable lack of transparency will be removed. Another limitation is that culture medium composition changes rapidly. Both types of media used in our study are not available any longer and have meanwhile been replaced by media with an, according to the manufacturers, "improved" composition.

Many ethical and practical challenges apply to human embryo research if compared to animal research. In The Netherlands, embryos cannot be created just for research purposes and most research is therefore performed on - scarce - surplus (and by definition low quality) embryos. Additionally, whereas in animal studies it is possible to keep nutritional, environmental and genetical conditions standardized, this is not possible in human research. Moreover, the shorter lifespan of mice makes it easier to study "longterm" effects of culture medium on the outcome. To be able to show a risk increase for long-term effects in humans induced by culture conditions, we would have to wait for 60 years, which is not a reasonable period in the field of ART with its rapidly changing culture conditions and technologies. Therefore, a need exists for identifying early markers of long-term health risks.

Although we believe that it is of vital importance that more studies will be conducted on long-term culture medium effects, we also acknowledge that studying "long-term" effects in human will get more complicated as the child grows older. Obviously, many other (confounding) nurture factors (such as nutrition, life style and exercise level) will play an increasing role in the child's or adolescent's weight, BMI and overall (cardiovascular) health. 
Also attrition bias plays a role in follow-up studies on culture medium effects on children born after ART. The first selection is of the patients who get a live born child versus the patients who do not, which might represent groups with different patient characteristics. second, not all parents and their children will participate in follow-up studies, which also may introduce selection bias. Drop-out of patients in follow-up studies as in our study (>50\% lost-to-follow-up) is not an exceptionally high percentage for long-term follow-up, but the risk of selective drop-out is present.

Other IVF-procedure related factors

It is known that subfertility in itself is an important determinant of adverse obstetric and perinatal outcome (Draper et al., 1999) such as pre-eclampsia, preterm birth and low birthweight (Bergh and Wennerholm, 2012). It is known that women suffering from polycystic ovary syndrome (PCOS) are subfertile and prone to develop metabolic syndrome (Pourteymour Fard Tabrizi et al., 2013). It might therefore be speculated that subfertile women constitute a subpopulation that is at greater risk of cardiovascular disease. Their subfertility might be "a symptom" of the underlying pathology such as poor vascularisation also present in the uterus. However, it has been reported that even in siblings from the same mother IVF children have poorer perinatal outcome than their non-IVF siblings (Pinborg et al., 2013). This suggests a role for the ART treatment as an origin for the observed differences.

Since a potential genetic risk increase for cardiovascular diseases (inherited from their mothers) cannot be altered, it is important to focus on preventing possibly additional 'hits' that will increase the offspring's lifetime cardiovascular risks by optimizing culture conditions. In this thesis, we focused on the culture medium effects on health of IVF children. Apart from culture medium variations, there are several other aspects of the IVF treatment that are of potential influence on the outcome such as the length of the embryo culture, number of embryos transferred and the optional cryopreservation of embryos. Also the hormonal stimulation used during a regular IVF procedure has been shown to affect the newborn's perinatal outcome negatively (Vidal et al., 2017).

\section{What is "normal"?}

Regarding the fact that IVF children have generally a lower weight at birth, an important question that remains is which of the two culture media groups we studied is to be considered closest to "normal" and which deviates. The answer depends on what group is considered the best reference group. Taking a population of non-IVF children as a control group might seem like a logical choice, although differences in population characteristics such as maternal age and underlying health problems might bias the outcomes. If an IVF-population is used as a control-group, this also induces a heterogenic population with 
patients treated for all kinds of different infertility causes and therefore also diverse patient characteristics. This is not a problem if an RCT is performed, but may induce problems in observational studies. The best non-RCT way therefore to analyse effects of embryo culture might be to compare IVF versus non-IVF siblings born from the same parents. It will depend on the research question which control group is the most appropriate to provide the desired answer in observational studies.

\section{Wider implications}

There is a growing concern about the safety of ART. From animal studies we know that exposures to an abnormal or suboptimal environment during preimplantation and pregnancy might predispose the individual to chronic diseases in later life. Comparable effects are suggested in human research. It is worrying that alterations in the culture medium, to which the embryo is exposed for only 2-3 days, can induce an increase in weight, BMI and truncal fat 9 years later. The normal and comparable cardiovascular results for the two culture medium groups are reassuring for this moment. However, the children may be too young to express measurable differences in cardiovascular development. The knowledge from animal studies and the increasing evidence in human studies should create awareness among health professionals. Furthermore, it underlines that culture conditions and culture medium composition should not be taken for granted. Robust evidence on which culture medium (ingredients) are superior for embryo culture is not available. Safety and attention for possible long-term health risks should prevail.

Although overall the health of IVF children is good and most children develop normally, prolonged follow-up and structured investigations up until adulthood are necessary to gain more insight and reassurance in the health and cardiovascular and cognitive development of IVF offspring.

Furthermore, knowledge from animal and human studies on long-term health effects of changes in the peri-implantation window should also become part of the regular preconception care of all women trying to conceive. They should be aware that specifically their nutrition, but also their behaviour (such as exposure to excessive stress) are already influencing their future child, possibly even before the embryo has implanted since also the oviductal fluid and uterine fluid compositions change with maternal nutrition (Fleming et al., 2004). 


\section{CONCLUSION AND FUTURE PROSPECTS}

It is important that the long-term safety of IVF is prioritized in research and further development of new techniques. While the development of embryo culture medium has focused mainly on increasing live birth rates and quality of embryos, knowledge about long-term health effects and possible risks is lacking, and the introduction of new media into clinical practice is often a chance event. It is of vital importance that culture media manufacturers make their media's exact composition publicly available for their end-users to make it possible to disentangle the mechanisms behind the differences induced by culture media. Both manufacturers and embryologists share the responsibility to carefully consider the safety and the possibility of life-long changes induced by the culture medium that is used. Clinicians and embryologists should make sure that the techniques not only increase the pregnancy chances of a couple, but they also should strive to document their effects on long-term health as well.

It is difficult to explain to patients the challenges clinicians and embryologists are facing, since it is still uncertain to what extend the risks for adult diseases are increased and what the etiology behind the observed differences is. Patients and (future) parents of IVF children may be reassured by the fact that IVF children are generally healthy and developing normally into young adults. However, they should also be informed about the fact that Louise Brown, the oldest IVF child on earth, just turned 40 years old and as a result it is impossible to predict what the lifelong health risks might imply.

Our group is currently performing another multicenter RCT in The Netherlands, comparing IVF results of culture using a sequential medium with a single-step medium. Furthermore, to analyse the possible epigenetic effects of culture medium, we are performing DNA methylation analysis on the saliva of the children from our prospective follow-up study. Also, we are performing DNA methylation analysis on cord blood from the children born from our first multicenter RCT comparing outcome of embryo culture in either HTF or G5 Vitrolife. Future research should focus on unravelling to which extent all individual factors contribute to the observed differences in the offspring and what the role of culture medium is in these differences. It is important that research focuses primarily on effects of culture conditions on the outcome of the IVF offspring, since this could possibly be altered and might then improve outcome. Furthermore, keeping structured databases with records of all IVF related factors (stimulation protocols, culture conditions, including the culture medium used, oxygen concentrations, numbers of embryos per drop, number of embryos transferred) should be introduced in daily practice and not only in time-consuming research projects. But most importantly, long-term follow up of children born after IVF should be part of the standard care in every IVF centre worldwide. 


\section{REFERENCES}

Baidal JAW, Locks LM, Cheng ER, Blake-Lamb TL, Perkins ME, Taveras EM. Risk Factors for Childhood Obesity in the First 1,000 Days A Systematic Review. Am J Prev Med 2016;50: 761-779.

Banrezes B, Sainte-Beuve T, Canon E, Schultz RM, Cancela J, Ozil JP. Adult body weight is programmed by a redox-regulated and energy-dependent process during the pronuclear stage in mouse. PLoS One 2011;6: e29388.

Barbuscia A, Mills MC. Cognitive development in children up to age 11 years born after ART-a longitudinal cohort study. Hum Reprod 2017: 1-7.

Barbuscia A, Mills MC. Cognitive development in children up to age 11 years born after ART-a longitudinal cohort study. Hum Reprod 2017;32: 1482-1488.

Barker DJ. Maternal nutrition, fetal nutrition, and disease in later life. Nutrition 1997;13: 807-813.

Barker DJ. Fetal origins of cardiovascular disease. Ann Med 1999;31 Suppl 1: 3-6.

Barker DJ. In utero programming of cardiovascular disease. Theriogenology 2000;53: 555-574.

Barker DJ. Adult consequences of fetal growth restriction. Clin Obstet Gynecol 2006;49: 270-283.

Barker DJP. The origins of the developmental origins theory. J Intern Med 2007;261: 412-417.

Basatemur E, Shevlin M, Sutcliffe A. Growth of children conceived by IVF and ICSI up to 12years of age. Reprod Biomed Online 2010;20: 144-149.

Bay B, Mortensen EL, Kesmodel US. Is subfertility or fertility treatment associated with long-term growth in the offspring? A cohort study. Fertil Steril 2014;102: 1117-1123.

Belfort MB, Gillman MW. Healthy infant growth: what are the trade-offs in the developed world? Nestle Nutr Inst Workshop Ser 2013;71: 171-184.

Belva F, Henriet S, Liebaers I, Van Steirteghem A, Celestin-Westreich S, Bonduelle M. Medical outcome of 8-year-old singleton ICSI children (born >or=32 weeks' gestation) and a spontaneously conceived comparison group. Hum Reprod 2007;22: 506-515.

Belva F, Painter R, Bonduelle M, Roelants M, Devroey P, De Schepper J. Are ICSI adolescents at risk for increased adiposity? Hum Reprod 2012;27: 257-264.

Bergh C, Wennerholm UB. Obstetric outcome and long-term follow up of children conceived through assisted reproduction. Best Pract Res Clin Obstet Gynaecol 2012;26: 841-852.

Berry KA, Baron IS, Weiss BA, Baker R, Ahronovich MD, Litman FR. In vitro fertilization and late preterm preschoolers' neuropsychological outcomes: the PETIT study. Am J Obstet Gynecol 2013;209: 356.e351-356.

Bloise E, Feuer SK, Rinaudo PF. Comparative intrauterine development and placental function of ART concepti: implications for human reproductive medicine and animal breeding. Human reproduction update 2014;20: 822-839.

Bloise E, Lin W, Liu X, Simbulan R, Kolahi KS, Petraglia F, Maltepe E, Donjacour A, Rinaudo P. Impaired placental nutrient transport in mice generated by in vitro fertilization. Endocrinology 2012;153: 3457-3467.

Boivin J, Bunting L, Collins JA, Nygren KG. International estimates of infertility prevalence and treatment-seeking: potential need and demand for infertility medical care. Hum Reprod 2007;22: 1506-1512.

Bouillon C, Léandri R, Desch L, Ernst A, Bruno C, Cerf C, Chiron A, Souchay C, Burguet A, Jimenez C et al. Does Embryo Culture Medium Influence the Health and Development of Children Born after In Vitro Fertilization? PLoS One 2016;11: e0150857

Bowman P, McLaren A. Viability and growth of mouse embryos after in vitro culture and fusion. Journal of embryology and experimental morphology 1970;23: 693-704.

Brandes RP. Endothelial dysfunction and hypertension. Hypertension 2014;64: 924-928.

Calle A, Fernandez-Gonzalez R, Ramos-Ibeas P, Laguna-Barraza R, Perez-Cerezales S, Bermejo-Alvarez P, Ramirez MA, Gutierrez-Adan A. Long-term and transgenerational effects of in vitro culture on mouse embryos. Theriogenology 2012;77: 785-793. 
Carling SJ, Demment MM, Kjolhede CL, Olson CM. Breastfeeding duration and weight gain trajectory in infancy. Pediatrics 2015;135: 111-119.

Carrasco B, Boada M, Rodríguez I, Coroleu B, Barri PN, Veiga A. Does culture medium influence offspring birth weight? Fertil Steril 2013;100: 1283-1288.

CBS. https://www.onderwijsincijfers.n//kengetallen/sectoroverstijgend/nederlands-onderwijsstelsel/hoogst-behaalde-onderwijsniveau.

Ceelen M, van Weissenbruch MM, Prein J, Smit J, Vermeiden JP, Spreeuwenberg M, van Leeuwen FE, Delemarre-van de Waal HA. Growth during infancy and early childhood in relation to blood pressure and body fat measures at age 8-18 years of IVF children and spontaneously conceived controls born to subfertile parents. Hum Reprod 2009;24: 2788-2795.

Ceelen M, van Weissenbruch MM, Roos JC, Vermeiden JP, van Leeuwen FE, Delemarre-van de Waal HA. Body composition in children and adolescents born after in vitro fertilization or spontaneous conception. J Clin Endocrinol Metab 2007;92: 3417-3423.

Ceelen M, van Weissenbruch MM, Vermeiden JP, van Leeuwen FE, Delemarre-van de Waal HA. Cardiometabolic differences in children born after in vitro fertilization: follow-up study. J Clin Endocrino/ Metab 2008;93: 1682-1688.

Ceelen M, van Weissenbruch MM, Vermeiden JP, van Leeuwen FE, Delemarre-van de Waal HA. Growth and development of children born after in vitro fertilization. Fertil Steril 2008;90: 1662-1673.

Chen M, Wu L, Zhao J, Wu F, Davies MJ, Wittert GA, Norman RJ, Robker RL, Heilbronn LK. Altered glucose metabolism in mouse and humans conceived by IVF. Diabetes 2014;63: 3189-3198.

Chin-Lun Hung G, Hahn J, Alamiri B, Buka SL, Goldstein JM, Laird N, Nelson CA, Smoller JW, Gilman SE. Socioeconomic disadvantage and neural development from infancy through early childhood. Int J Epidemiol 2015;44: 1889-1899.

Chiolero A, Kaufman JS, Paradis G. Why adjustment for current weight can bias the estimate of the effect of birth weight on blood pressure: shedding light using causal graphs. J Hypertens 2012;30: 1042-1045.

Chronopoulou E, Harper JC. IVF culture media: past, present and future. Hum Reprod Update 2015;21: 39-55.

CITO. 2016, http://www.onderwijsadvies.n//media/889059/vaardigheidsscores-en-niveau-a-e-v8.pdf.

Cole TJ, Lobstein T. Extended international (IOTF) body mass index cut-offs for thinness, overweight and obesity. Pediatr Obes 2012;7: 284-294

Cracowski JL, Minson CT, Salvat-Melis M, Halliwill JR. Methodological issues in the assessment of skin microvascular endothelial function in humans. Trends Pharmacol Sci 2006;27: 503-508.

De Vos A, Janssens R, Van de Velde H, Haentjens P, Bonduelle M, Tournaye H, Verheyen G. The type of culture medium and the duration of in vitro culture do not influence birthweight of ART singletons. Hum Reprod 2015;30: 20-27.

Delle Piane L, Lin W, Liu X, Donjacour A, Minasi P, Revelli A, Maltepe E, Rinaudo PF. Effect of the method of conception and embryo transfer procedure on mid-gestation placenta and fetal development in an IVF mouse model. Human reproduction 2010;25: 2039-2046

Doherty AS, Mann MRW, Tremblay KD, Bartolomei MS, Schultz RM. Differential effects of culture on imprinted H19 expression in the preimplantation mouse embryo. Biol Reprod 2000;62: 1526-1535.

Donjacour A, Liu X, Lin W, Simbulan R, Rinaudo PF. In vitro fertilization affects growth and glucose metabolism in a sex-specific manner in an outbred mouse model. Biol Reprod 2014;90: 80.

Draper ES, Kurinczuk J, Abrams KR, Clarke M. Assessment of separate contributions to perinatal mortality of infertility history and treatment: a case-control analysis. Lancet 1999;353: 1746-1749.

Dumoulin JC, Land JA, Van Montfoort AP, Nelissen EC, Coonen E, Derhaag JG, Schreurs IL, Dunselman GA, Kester AD, Geraedts JP et al. Effect of in vitro culture of human embryos on birthweight of newborns. Hum Reprod 2010;25: 605-612. 
Dyrlund TF, Kirkegaard K, Poulsen ET, Sanggaard KW, Hindkjaer JJ, Kjems J, Enghild JJ, Ingerslev HJ. Unconditioned commercial embryo culture media contain a large variety of non-declared proteins: a comprehensive proteomics analysis. Hum Reprod 2014;29: 2421-2430.

Eaton JL, Lieberman ES, Stearns C, Chinchilla M, Racowsky C. Embryo culture media and neonatal birthweight following IVF. Hum Reprod 2012;27: 375-379.

Ecker DJ, Stein P, Xu Z, Williams CJ, Kopf GS, Bilker WB, Abel T, Schultz RM. Long-term effects of culture of preimplantation mouse embryos on behavior. Proceedings of the National Academy of Sciences of the United States of America 2004;101: 1595-1600.

Eckert JJ, Porter R, Watkins AJ, Burt E, Brooks S, Leese HJ, Humpherson PG, Cameron IT, Fleming TP. Metabolic induction and early responses of mouse blastocyst developmental programming following maternal low protein diet affecting life-long health. PLoS One 2012; 7 : e52791.

Eriksson J, Forsén T, Tuomilehto J, Osmond C, Barker D. Size at birth, childhood growth and obesity in adult life. Int J Obes Relat Metab Disord 2001;25: 735-740.

Eriksson JG, Forsén T, Tuomilehto J, Osmond C, Barker DJ. Early adiposity rebound in childhood and risk of Type 2 diabetes in adult life. Diabetologia 2003;46: 190-194.

Eskild A, Monkerud L, Tanbo T. Birthweight and placental weight; do changes in culture media used for IVF matter? Comparisons with spontaneous pregnancies in the corresponding time periods. Hum Reprod 2013;28: 3207 3214.

Evers JL. Female subfertility. Lancet 2002;360: 151-159.

Fabricius-Bjerre S, Jensen RB, Faerch K, Larsen T, Molgaard C, Michaelsen KF, Vaag A, Greisen G. Impact of birth weight and early infant weight gain on insulin resistance and associated cardiovascular risk factors in adolescence. PLoS One 2011;6: e20595.

Faddy MJ, Gosden MD, Gosden RG. A demographic projection of the contribution of assisted reproductive technologies to world population growth. Reprod Biomed Online 2018;36: 455-458.

Feelisch M. The use of nitric oxide donors in pharmacological studies. Naunyn Schmiedebergs Arch Pharmacol 1998;358: 113-122.

Fernández-Gonzalez R, Moreira P, Bilbao A, Jiménez A, Pérez-Crespo M, Ramírez MA, Rodríguez De Fonseca F, Pintado B, Gutiérrez-Adán A. Long-term effect of in vitro culture of mouse embryos with serum on mRNA expression of imprinting genes, development, and behavior. Proc Natl Acad Sci U S A 2004;101: 5880-5885.

Feuer S, Rinaudo P. Preimplantation stress and development. Birth Defects Res C Embryo Today 2012;96: 299-314.

Feuer SK, Liu X, Donjacour A, Lin W, Simbulan RK, Giritharan G, Piane LD, Kolahi K, Ameri K, Maltepe E et al. Use of a mouse in vitro fertilization model to understand the developmental origins of health and disease hypothesis. Endocrinology 2014;155: 1956-1969.

Feuer SK, Liu X, Donjacour A, Lin W, Simbulan RK, Giritharan G, Piane LD, Kolahi K, Ameri K, Maltepe E et al. Use of a Mouse in Vitro Fertilization Model to Understand the Developmental Origins of Health and Disease Hypothesis. Endocrinology 2014: en20132081.

Fleming TP, Kwong WY, Porter R, Ursell E, Fesenko I, Wilkins A, Miller DJ, Watkins AJ, Eckert JJ. The embryo and its future. Biol Reprod 2004;71: 1046-1054.

Fleming TP, Watkins AJ, Velazquez MA, Mathers JC, Prentice AM, Stephenson J, Barker M, Saffery R, Yajnik CS, Eckert $\mathrm{J}$ et al. Origins of lifetime health around the time of conception: causes and consequences. Lancet 2018.

Gardner DK, Hamilton R, McCallie B, Schoolcraft WB, Katz-Jaffe MG. Human and mouse embryonic development, metabolism and gene expression are altered by an ammonium gradient in vitro. Reproduction 2013;146: 49-61.

Gardner DK, Kelley RL. Impact of the IVF laboratory environment on human preimplantation embryo phenotype. J Dev Orig Health Dis 2017;8: 418-435.

Gardner DK, Lane M. Culture and selection of viable blastocysts: a feasible proposition for human IVF? Hum Reprod Update 1997;3: 367-382. 
Gardner DK, Leese HJ. Concentrations of nutrients in mouse oviduct fluid and their effects on embryo development and metabolism in vitro. J Reprod Fertil 1990;88: 361-368.

Gibbons WE, Cedars M, Ness RB, Society for Assisted Reproductive Technologies Writing G. Toward understanding obstetrical outcome in advanced assisted reproduction: varying sperm, oocyte, and uterine source and diagnosis. Fertility and sterility 2011;95: 1645-1649 e1641.

Gillman MW, Barker D, Bier D, Cagampang F, Challis J, Fall C, Godfrey K, Gluckman P, Hanson M, Kuh D et al. Meeting report on the 3rd International Congress on Developmental Origins of Health and Disease (DOHaD). Pediatric research 2007;61: 625-629.

Giritharan G, Talbi S, Donjacour A, Di Sebastiano F, Dobson AT, Rinaudo PF. Effect of in vitro fertilization on gene expression and development of mouse preimplantation embryos. Reproduction 2007;134: 63-72.

Gluckman PD, Hanson MA, Bateson P, Beedle AS, Law CM, Bhutta ZA, Anokhin KV, Bougneres P, Chandak GR, Dasgupta $\mathrm{P}$ et al. Towards a new developmental synthesis: adaptive developmental plasticity and human disease. Lancet 2009;373: 1654-1657.

Godfrey KM, Gluckman PD, Hanson MA. Developmental origins of metabolic disease: life course and intergenerational perspectives. Trends in endocrinology and metabolism: TEM 2010;21: 199-205.

Green MP, Mouat F, Miles HL, Hopkins SA, Derraik JG, Hofman PL, Peek JC, Cutfield WS. Phenotypic differences in children conceived from fresh and thawed embryos in in vitro fertilization compared with naturally conceived children. Fertil Steril 2013;99: 1898-1904.

Griesinger G, Kolibianakis EM, Diedrich K, Ludwig M. Ovarian stimulation for IVF has no quantitative association with birthweight: a registry study. Human reproduction 2008;23: 2549-2554.

Gruber I, Klein M. Embryo culture media for human IVF: which possibilities exist? J Turk Ger Gynecol Assoc 2011;12: 110-117.

Gu F, Deng M, Gao J, Wang Z, Ding C, Xu Y, Zhou C. The effects of embryo culture media on the birthweight of singletons via fresh or frozen-thawed embryo transfer: a large-scale retrospective study. BMC Pregnancy Childbirth 2016;16: 270.

Guo XY, Liu XM, Jin L, Wang TT, Ullah K, Sheng JZ, Huang HF. Cardiovascular and metabolic profiles of offspring conceived by assisted reproductive technologies: a systematic review and meta-analysis. Fertil Steril 2017;107: 622-631 e625.

Han HD, Kiessling AA. In vivo development of transferred mouse embryos conceived in vitro in simple and complex media. Fertility and sterility 1988;50: 159-163.

Hart R, Norman RJ. The longer-term health outcomes for children born as a result of IVF treatment: Part I--General health outcomes. Hum Reprod Update 2013;19: 232-243.

Hart R, Norman RJ. The longer-term health outcomes for children born as a result of IVF treatment. Part II--Mental health and development outcomes. Hum Reprod Update 2013;19: 244-250.

Hassani F, Eftekhari-Yazdi P, Karimian L, Rezazadeh Valojerdi M, Movaghar B, Fazel M, Fouladi HR, Shabani F, Johansson L. The Effects of ISM1 Medium on Embryo Quality and Outcomes of IVF/ICSI Cycles. International journal of fertility \& sterility 2013;7: 108-115.

Helmerhorst FM, Perquin DA, Donker D, Keirse MJ. Perinatal outcome of singletons and twins after assisted conception: a systematic review of controlled studies. Bmj 2004;328: 261.

Henningsen AK, Pinborg A. Birth and perinatal outcomes and complications for babies conceived following ART. Semin Fetal Neonatal Med 2014;19: 234-238.

Henningsen AK, Pinborg A, Lidegaard O, Vestergaard C, Forman JL, Andersen AN. Perinatal outcome of singleton siblings born after assisted reproductive technology and spontaneous conception: Danish national sibling-cohort study. Fertil Steril 2011;95: 959-963.

Hentemann M, Bertheussen K. New media for culture to blastocyst. Fertil Steril 2009;91: 878-883. 
Hokken-Koelega AC, De Ridder MA, Lemmen RJ, Den Hartog H, De Muinck Keizer-Schrama SM, Drop SL. Children born small for gestational age: do they catch up? Pediatr Res 1995;38: 267-271.

Imudia AN, Awonuga AO, Kaimal AJ, Wright DL, Styer AK, Toth TL. Elective cryopreservation of all embryos with subsequent cryothaw embryo transfer in patients at risk for ovarian hyperstimulation syndrome reduces the risk of adverse obstetric outcomes: a preliminary study. Fertility and sterility 2013;99: 168-173.

Jackson RA, Gibson KA, Wu YW, Croughan MS. Perinatal outcomes in singletons following in vitro fertilization: a meta-analysis. Obstet Gynecol 2004;103: 551-563.

Johnson TS, Engstrom JL, Warda JA, Kabat M, Peters B. Reliability of length measurements in full-term neonates. J Obstet Gynecol Neonatal Nurs 1998;27: 270-276.

Kagura J, Adair LS, Munthali RJ, Pettifor JM, Norris SA. Association Between Early Life Growth and Blood Pressure Trajectories in Black South African Children. Hypertension 2016;68: 1123-1131.

Khosla S, Dean W, Reik W, Feil R. Culture of preimplantation embryos and its long-term effects on gene expression and phenotype. Human reproduction update 2001;7: 419-427.

Khuc K, Blanco E, Burrows R, Reyes M, Castillo M, Lozoff B, Gahagan S. Adolescent metabolic syndrome risk is increased with higher infancy weight gain and decreased with longer breast feeding. Int J Pediatr 2012;2012: 478610.

Kleijkers SH, Mantikou E, Slappendel E, Consten D, van Echten-Arends J, Wetzels AM, van Wely M, Smits LJ, van Montfoort AP, Repping S et al. Influence of embryo culture medium (G5 and HTF) on pregnancy and perinatal outcome after IVF: a multicenter RCT. Hum Reprod 2016;31: 2219-2230.

Kleijkers SH, van Montfoort AP, Bekers O, Coonen E, Derhaag JG, Evers JL, Dumoulin JC. Ammonium accumulation in commercially available embryo culture media and protein supplements during storage at 2-8 degrees C and during incubation at 37 degrees C. Hum Reprod 2016;31: 1192-1199.

Kleijkers SH, van Montfoort AP, Smits LJ, Coonen E, Derhaag JG, Evers JL, Dumoulin JC. Age of G-1 PLUS v5 embryo culture medium is inversely associated with birthweight of the newborn. Hum Reprod 2015;30: 1352-1357.

Kleijkers SH, van Montfoort AP, Smits LJ, Viechtbauer W, Roseboom TJ, Nelissen EC, Coonen E, Derhaag JG, Bastings

$L$, Schreurs IE et al. IVF culture medium affects post-natal weight in humans during the first 2 years of life. Hum Reprod 2014;29: 661-669.

Koivurova S, Hartikainen AL, Sovio U, Gissler M, Hemminki E, Jarvelin MR. Growth, psychomotor development and morbidity up to 3 years of age in children born after IVF. Hum Reprod 2003;18: 2328-2336.

Krishnaveni GV, Veena SR, Wills AK, Hill JC, Karat SC, Fall CH. Adiposity, insulin resistance and cardiovascular risk factors in 9-10-year-old Indian children: relationships with birth size and postnatal growth. J Dev Orig Health Dis 2010;1: 403-411.

Kuiper D, Hoek A, la Bastide-van Gemert S, Seggers J, Mulder DJ, Haadsma M, Heineman MJ, Hadders-Algra M. Cardiovascular health of 9-year-old IVF offspring: no association with ovarian hyperstimulation and the in vitro procedure. Hum Reprod 2017;32: 2540-2548.

Lane M, Gardner DK. Ammonium induces aberrant blastocyst differentiation, metabolism, pH regulation, gene expression and subsequently alters fetal development in the mouse. Biol Reprod 2003;69: 1109-1117.

Lawitts JA, Biggers JD. Optimization of mouse embryo culture media using simplex methods. J Reprod Fertil 1991;91: 543-556.

Lee SH, Lee MY, Chiang TL, Lee MS, Lee MC. Child growth from birth to 18 months old born after assisted reproductive technology--results of a national birth cohort study. Int J Nurs Stud 2010;47: 1159-1166.

Lei $X$, Chen Y, Ye J, Ouyang F, Jiang F, Zhang J. The optimal postnatal growth trajectory for term small for gestational age babies: a prospective cohort study. J Pediatr 2015;166: 54-58.

Lemmen JG, Pinborg A, Rasmussen S, Ziebe S. Birthweight distribution in ART singletons resulting from embryo culture in two different culture media compared with the national population. Human reproduction 2014;29: 2326-2332. 
Leunissen RW, Kerkhof GF, Stijnen T, Hokken-Koelega A. Timing and tempo of first-year rapid growth in relation to cardiovascular and metabolic risk profile in early adulthood. JAMA 2009;301: 2234-2242.

Leunissen RW, Stijnen T, Hokken-Koelega AC. Influence of birth size on body composition in early adulthood: the programming factors for growth and metabolism (PROGRAM)-study. Clin Endocrinol (Oxf) 2009;70: 245-251.

Lim D, Bowdin SC, Tee L, Kirby GA, Blair E, Fryer A, Lam W, Oley C, Cole T, Brueton LA et al. Clinical and molecular genetic features of Beckwith-Wiedemann syndrome associated with assisted reproductive technologies. Human reproduction 2009;24: 741-747.

Lin S, Li M, Lian Y, Chen L, Liu P. No effect of embryo culture media on birthweight and length of newborns. Hum Reprod 2013;28: 1762-1767.

Lv PP, Meng Y, Lv M, Feng C, Liu Y, Li JY, Yu DQ, Shen Y, Hu XL, Gao Q et al. Altered thyroid hormone profile in offspring after exposure to high estradiol environment during the first trimester of pregnancy: a cross-sectional study. BMC Med 2014;12: 240.

MacLaughlin SM, Walker SK, Roberts CT, Kleemann DO, McMillen IC. Periconceptional nutrition and the relationship between maternal body weight changes in the periconceptional period and feto-placental growth in the sheep. J Physiol 2005;565: 111-124

Mains L, Zimmerman M, Blaine J, Stegmann B, Sparks A, Ansley T, Van Voorhis B. Achievement test performance in children conceived by IVF. Hum Reprod 2010;25: 2605-2611.

Makinen S, Soderstrom-Anttila V, Vainio J, Suikkari AM, Tuuri T. Does long in vitro culture promote large for gestational age babies? Hum Reprod 2013;28: 828-834

Malchau SS, Loft A, Larsen EC, Aaris Henningsen AK, Rasmussen S, Andersen AN, Pinborg A. Perinatal outcomes in 375 children born after oocyte donation: a Danish national cohort study. Fertility and sterility 2013;99: 1637-1643.

Marino JL, Moore VM, Willson KJ, Rumbold A, Whitrow MJ, Giles LC, Davies MJ. Perinatal outcomes by mode of assisted conception and sub-fertility in an Australian data linkage cohort. PloS one 2014;9: e80398.

Market-Velker BA, Fernandes AD, Mann MR. Side-by-side comparison of five commercial media systems in a mouse model: suboptimal in vitro culture interferes with imprint maintenance. Biol Reprod 2010;83: 938-950.

Matte TD, Bresnahan M, Begg MD, Susser E. Influence of variation in birth weight within normal range and within sibships on IQ at age 7 years: cohort study. BMJ 2001;323: 310-314.

McDonald SD, Han Z, Mulla S, Murphy KE, Beyene J, Ohlsson A, Knowledge Synthesis G. Preterm birth and low birth weight among in vitro fertilization singletons: a systematic review and meta-analyses. Eur J Obstet Gynecol Reprod Biol 2009;146: 138-148.

Messerlian C, Maclagan L, Basso O. Infertility and the risk of adverse pregnancy outcomes: a systematic review and meta-analysis. Human reproduction 2013;28: 125-137.

Meyer JS, Novak MA. Minireview: Hair cortisol: a novel biomarker of hypothalamic-pituitary-adrenocortical activity. Endocrinology 2012;153: 4120-4127.

Morbeck DE, Krisher RL, Herrick JR, Baumann NA, Matern D, Moyer T. Composition of commercial media used for human embryo culture. Fertil Steril 2014;102: 759-766 e759.

Morbeck DE, Paczkowski M, Fredrickson JR, Krisher RL, Hoff HS, Baumann NA, Moyer T, Matern D. Composition of protein supplements used for human embryo culture. J Assist Reprod Genet 2014;31: 1703-1711.

Murray E, Pearson R, Fernandes M, Santos IS, Barros FC, Victora CG, Stein A, Matijasevich A. Are fetal growth impairment and preterm birth causally related to child attention problems and ADHD? Evidence from a comparison between high-income and middle-income cohorts. J Epidemiol Community Health 2016;70: 704-709.

Nakashima A, Araki R, Tani H, Ishihara O, Kuwahara A, Irahara M, Yoshimura Y, Kuramoto T, Saito H, Nakaza A et al. Implications of assisted reproductive technologies on term singleton birth weight: an analysis of 25,777 children in the national assisted reproduction registry of Japan. Fertility and sterility 2013;99: 450-455. 
National. High Blood Pressure Education Program Working Group on High Blood Pressure in Children and Adolescents. The fourth report on the diagnosis, evaluation, and treatment of high blood pressure in children and adolescents. Pediatrics 2004;114: 555-576.

Nelissen EC, Van Montfoort AP, Coonen E, Derhaag JG, Geraedts JP, Smits LJ, Land JA, Evers JL, Dumoulin JC. Further evidence that culture media affect perinatal outcome: findings after transfer of fresh and cryopreserved embryos. Human reproduction 2012;27: 1966-1976.

Nelissen EC, Van Montfoort AP, Smits LI, Menheere PP, Evers JL, Coonen E, Derhaag JG, Peeters LL, Coumans AB, Dumoulin JC. IVF culture medium affects human intrauterine growth as early as the second trimester of pregnancy. Hum Reprod 2013;28: 2067-2074.

Onal H, Ercan O, Adal E, Ersen A, Onal Z. Subclinical hypothyroidism in in vitro fertilization babies. Acta Paediatr 2012;101: e248-252.

Palermo G, Joris H, Devroey P, Van Steirteghem AC. Pregnancies after intracytoplasmic injection of single spermatozoon into an oocyte. Lancet 1992;340: 17-18.

Pandey S, Shetty A, Hamilton M, Bhattacharya S, Maheshwari A. Obstetric and perinatal outcomes in singleton pregnancies resulting from IVF/ICSI: a systematic review and meta-analysis. Hum Reprod Update 2012;18: 485-503.

Patro-Golab B, Zalewski BM, Kouwenhoven SM, Karas J, Koletzko B, Bernard van Goudoever J, Szajewska H. Protein Concentration in Milk Formula, Growth, and Later Risk of Obesity: A Systematic Review. J Nutr 2016;146: 551-564.

Pelinck MJ, Hadders-Algra M, Haadsma ML, Nijhuis WL, Kiewiet SM, Hoek A, Heineman MJ, Middleburg KJ. Is the birthweight of singletons born after IVF reduced by ovarian stimulation or by IVF laboratory procedures? Reproductive biomedicine online 2010;21: 245-251.

Pinborg A, Henningsen AA, Loft A, Malchau SS, Forman J, Andersen AN. Large baby syndrome in singletons born after frozen embryo transfer (FET): is it due to maternal factors or the cryotechnique? Hum Reprod 2014;29: 618-627.

Pinborg A, Wennerholm UB, Romundstad LB, Loft A, Aittomaki K, Söderström-Anttila V, Nygren KG, Hazekamp J, Bergh C. Why do singletons conceived after assisted reproduction technology have adverse perinatal outcome? Systematic review and meta-analysis. Hum Reprod Update 2013;19: 87-104.

Pontesilli M, Painter RC, Grooten IJ, van der Post JA, Mol BW, Vrijkotte TG, Repping S, Roseboom TJ. Subfertility and assisted reproduction techniques are associated with poorer cardiometabolic profiles in childhood. Reprod Biomed Online 2015;30: 258-267.

Pourteymour Fard Tabrizi F, Alipoor B, Mehrzad Sadaghiani M, Ostadrahimi A, Malek Mahdavi A. Metabolic Syndrome and Its Characteristics among Reproductive-Aged Women with Polycystic Ovary Syndrome: A Cross-sectional Study in Northwest Iran. Int J Fertil Steril 2013;6: 244-249.

Puissant C, Abraham P, Durand S, Humeau-Heurtier A, Faure S, Leftheriotis G, Rousseau P, Mahe G. Reproducibility of non-invasive assessment of skin endothelial function using laser Doppler flowmetry and laser speckle contrast imaging. PLoS One 2013;8: e61320.

Rexhaj E, Paoloni-Giacobino A, Rimoldi SF, Fuster DG, Anderegg M, Somm E, Bouillet E, Allemann Y, Sartori C, Scherrer U. Mice generated by in vitro fertilization exhibit vascular dysfunction and shortened life span.J Clin Invest 2013;123: 5052-5060.

Rimoldi SF, Sartori C, Rexhaj E, Bailey DM, de Marchi SF, McEneny J, Arx R, Cerny D, Duplain H, Germond M et al. Antioxidants improve vascular function in children conceived by assisted reproductive technologies: A randomized double-blind placebo-controlled trial. EurJ Prev Cardiol 2015;22: 1399-1407.

Rimoldi SF, Sartori C, Rexhaj E, Cerny D, Von Arx R, Soria R, Germond M, Allemann Y, Scherrer U. Vascular dysfunction in children conceived by assisted reproductive technologies: underlying mechanisms and future implications. Swiss Med Wkly 2014;144: w13973.

Roberts SA, Vail A. On the appropriate interpretation of evidence: the example of culture media and birth weight. Hum Reprod 2017;32: 1151-1154. 
Romundstad LB, Romundstad PR, Sunde A, von During V, Skjaerven R, Gunnell D, Vatten LJ. Effects of technology or maternal factors on perinatal outcome after assisted fertilisation: a population-based cohort study. Lancet 2008;372: 737-743.

Rooke JA, McEvoy TG, Ashworth CJ, Robinson JJ, Wilmut I, Young LE, Sinclair KD. Ovine fetal development is more sensitive to perturbation by the presence of serum in embryo culture before rather than after compaction. Theriogenology 2007;67: 639-647.

Roseboom T, de Rooij S, Painter R. The Dutch famine and its long-term consequences for adult health. Early Hum Dev 2006;82: 485-491

Roseboom TJ, Painter RC, van Abeelen AF, Veenendaal MV, de Rooij SR. Hungry in the womb: what are the consequences? Lessons from the Dutch famine. Maturitas 2011;70: 141-145.

Roseboom TJ, van der Meulen JH, van Montfrans GA, Ravelli AC, Osmond C, Barker DJ, Bleker OP. Maternal nutrition during gestation and blood pressure in later life. J Hypertens 2001;19: 29-34.

Ruijsbroek A, Wijga AH, Gehring U, Kerkhof M, Droomers M. School Performance: A Matter of Health or Socio-Economic Background? Findings from the PIAMA Birth Cohort Study. PLoS One 2015;10: e0134780.

Rumball CW, Bloomfield FH, Oliver MH, Harding JE. Different periods of periconceptional undernutrition have different effects on growth, metabolic and endocrine status in fetal sheep. Pediatr Res 2009;66: 605-613.

Rumbold AR, Moore VM, Whitrow MJ, Oswald TK, Moran LJ, Fernandez RC, Barnhart KT, Davies MJ. The impact of specific fertility treatments on cognitive development in childhood and adolescence: a systematic review. Hum Reprod 2017;32: 1489-1507.

Sakka SD, Loutradis D, Kanaka-Gantenbein C, Margeli A, Papastamataki M, Papassotiriou I, Chrousos GP. Absence of insulin resistance and low-grade inflammation despite early metabolic syndrome manifestations in children born after in vitro fertilization. Fertil Steril 2010;94: 1693-1699.

Sauvé B, Koren G, Walsh G, Tokmakejian S, Van Uum SH. Measurement of cortisol in human hair as a biomarker of systemic exposure. Clin Invest Med 2007;30: E183-191.

Savage JS, Birch LL, Marini M, Anzman-Frasca S, Paul IM. Effect of the INSIGHT Responsive Parenting Intervention on Rapid Infant Weight Gain and Overweight Status at Age 1 Year: A Randomized Clinical Trial. JAMA Pediatr 2016;170: 742-749.

Sazonova A, Kallen K, Thurin-Kjellberg A, Wennerholm UB, Bergh C. Obstetric outcome in singletons after in vitro fertilization with cryopreserved/thawed embryos. Human reproduction 2012;27: 1343-1350.

Schellong K, Schulz S, Harder T, Plagemann A. Birth weight and long-term overweight risk: systematic review and a meta-analysis including 643,902 persons from 66 studies and 26 countries globally. PLoS One 2012;7: e47776.

Schendelaar P, La Bastide-Van Gemert S, Heineman MJ, Middelburg KJ, Seggers J, Van den Heuvel ER, Hadders-Algra M. Subfertility factors rather than assisted conception factors affect cognitive and behavioural development of 4-year-old singletons. Reprod Biomed Online 2016;33: 752-762.

Scherrer U, Rimoldi SF, Rexhaj E, Stuber T, Duplain H, Garcin S, de Marchi SF, Nicod P, Germond M, Allemann Y et al. Systemic and pulmonary vascular dysfunction in children conceived by assisted reproductive technologies. Circulation 2012;125: 1890-1896.

Schieve LA, Meikle SF, Ferre C, Peterson HB, Jeng G, Wilcox LS. Low and very low birth weight in infants conceived with use of assisted reproductive technology. The New England journal of medicine 2002;346: 731-737.

Schonbeck Y, Talma H, van Dommelen P, Bakker B, Buitendijk SE, HiraSing RA, van Buuren S. The world's tallest nation has stopped growing taller: the height of Dutch children from 1955 to 2009. Pediatr Res 2013;73: 371-377.

Schönbeck Y, Talma H, van Dommelen P, Bakker B, Buitendijk SE, Hirasing RA, van Buuren S. Increase in prevalence of overweight in Dutch children and adolescents: a comparison of nationwide growth studies in 1980, 1997 and 2009. PLoS One 2011;6: e27608. 
Schwarzer C, Esteves TC, Arauzo-Bravo MJ, Le Gac S, Nordhoff V, Schlatt S, Boiani M. ART culture conditions change the probability of mouse embryo gestation through defined cellular and molecular responses. Human reproduction 2012;27: 2627-2640.

Scott KA, Yamazaki Y, Yamamoto M, Lin Y, Melhorn SJ, Krause EG, Woods SC, Yanagimachi R, Sakai RR, Tamashiro KL. Glucose parameters are altered in mouse offspring produced by assisted reproductive technologies and somatic cell nuclear transfer. Biol Reprod 2010;83: 220-227.

Sinclair KD, McEvoy TG, Carolan C, Maxfield EK, Maltin CA, Young LE, Wilmut I, Robinson J, Broadbent PJ. Conceptus growth and development following in vitro culture of ovine embryos in media supplemented with bovine sera. Theriogenology 1998;49: 218-218.

Singhal A. Long-Term Adverse Effects of Early Growth Acceleration or Catch-Up Growth. Ann Nutr Metab 2017;70: 236-240.

Singhal A, Cole TJ, Fewtrell M, Deanfield J, Lucas A. Is slower early growth beneficial for long-term cardiovascular health? Circulation 2004;109: 1108-1113.

Singhal A, Lucas A. Early origins of cardiovascular disease: is there a unifying hypothesis? Lancet 2004;363: 16421645.

Skovlund E, Fenstad GU. Should we always choose a nonparametric test when comparing two apparently nonnormal distributions? J Clin Epidemio/ 2001;54: 86-92.

Spangmose AL, Malchau SS, Schmidt L, Vassard D, Rasmussen S, Loft A, Forman J, Pinborg A. Academic performance in adolescents born after ART-a nationwide registry-based cohort study. Hum Reprod 2017;32: 447-456.

Stalder T, Kirschbaum C, Alexander N, Bornstein SR, Gao W, Miller R, Stark S, Bosch JA, Fischer JE. Cortisol in hair and the metabolic syndrome. J Clin Endocrino/ Metab 2013;98: 2573-2580.

Steptoe PC, Edwards RG. Birth after the reimplantation of a human embryo. Lancet 1978;2: 366.

Summers MC, Biggers JD. Chemically defined media and the culture of mammalian preimplantation embryos: historical perspective and current issues. Hum Reprod Update 2003;9: 557-582.

Sunde A, Brison D, Dumoulin J, Harper J, Lundin K, Magli MC, Van den Abbeel E, Veiga A. Time to take human embryo culture seriously. Hum Reprod 2016;31: 2174-2182.

Taine M, Stengel B, Forhan A, Carles S, Botton J, Charles MA, Heude B, Group EM-CCS. Rapid Early Growth May Modulate the Association Between Birth Weight and Blood Pressure at 5 Years in the EDEN Cohort Study. Hypertension 2016;68: 859-865.

Thompson JG, Gardner DK, Pugh PA, McMillan WH, Tervit HR. Lamb birth weight is affected by culture system utilized during in vitro pre-elongation development of ovine embryos. Biol Reprod 1995;53: 1385-1391.

Thouas GA, Jones GM, Trounson AO. The 'GO' system--a novel method of microculture for in vitro development of mouse zygotes to the blastocyst stage. Reproduction 2003;126: 161-169.

Touwslager RN, Gerver WJ, Tan FE, Gielen M, Zeegers MP, Zimmermann LJ, Houben AJ, Blanco CE, Stehouwer CD, Mulder AL. Influence of growth during infancy on endothelium-dependent vasodilatation at the age of 6 months. Hypertension 2012;60: 1294-1300.

Touwslager RN, Houben AJ, Gielen M, Zeegers MP, Stehouwer CD, Zimmermann LJ, Kessels AG, Gerver WJ, Blanco CE, Mulder AL. Endothelial vasodilatation in newborns is related to body size and maternal hypertension. $J$ Hypertens 2012;30: 124-131.

Touwslager RN, Houben AJ, Tan FE, Gielen M, Zeegers MP, Stehouwer CD, Gerver WJ, Westerterp KR, Wouters L, Blanco CE et al. Growth and endothelial function in the first 2 years of life. J Pediatr 2015;166: 666-671 e661.

Turner J, Belch J, Khan F. Current concepts in assessment of microvascular endothelial function using laser Doppler imaging and iontophoresis. Trends Cardiovasc Med 2008;18: 109-116.

Twilhaar ES, de Kieviet JF, Aarnoudse-Moens CS, van Elburg RM, Oosterlaan J. Academic performance of children born preterm: a meta-analysis and meta-regression. Arch Dis Child Fetal Neonatal Ed 2017. 
Valenzuela-Alcaraz B, Crispi F, Bijnens B, Cruz-Lemini M, Creus M, Sitges M, Bartrons J, Civico S, Balasch J, Gratacos E. Assisted reproductive technologies are associated with cardiovascular remodeling in utero that persists postnatally. Circulation 2013;128: 1442-1450.

Veena SR, Hegde BG, Ramachandraiah S, Krishnaveni GV, Fall CH, Srinivasan K. Relationship between adiposity and cognitive performance in 9-10-year-old children in South India. Arch Dis Child 2014;99: 126-134.

Velazquez MA, Sheth B, Smith SJ, Eckert JJ, Osmond C, Fleming TP. Insulin and branched-chain amino acid depletion during mouse preimplantation embryo culture programmes body weight gain and raised blood pressure during early postnatal life. Biochim Biophys Acta 2017;1864: 590-600.

Vergouw CG, Kostelijk EH, Doejaaren E, Hompes PG, Lambalk CB, Schats R. The influence of the type of embryo culture medium on neonatal birthweight after single embryo transfer in IVF. Hum Reprod 2012;27: 2619-2626.

Vermeiden JP, Bernardus RE. Are imprinting disorders more prevalent after human in vitro fertilization or intracytoplasmic sperm injection? Fertil Steril 2013;99: 642-651.

Vidal M, Vellve K, Gonzalez-Comadran M, Robles A, Prat M, Torne M, Carreras R, Checa MA. Perinatal outcomes in children born after fresh or frozen embryo transfer: a Catalan cohort study based on 14,262 newborns. Fertil Steril 2017;107: 940-947.

Visser GH, Eilers PH, Elferink-Stinkens PM, Merkus HM, Wit JM. New Dutch reference curves for birthweight by gestational age. Early Hum Dev 2009;85: 737-744.

Vrooman LA, Bartolomei MS. Can assisted reproductive technologies cause adult-onset disease? Evidence from human and mouse. Reprod Toxicol 2017;68: 72-84.

Watkins AJ, Lucas ES, Wilkins A, Cagampang FR, Fleming TP. Maternal periconceptional and gestational low protein diet affects mouse offspring growth, cardiovascular and adipose phenotype at 1 year of age. PLoS One 2011;6: e28745.

Watkins AJ, Platt D, Papenbrock T, Wilkins A, Eckert J], Kwong WY, Osmond C, Hanson M, Fleming TP. Mouse embryo culture induces changes in postnatal phenotype including raised systolic blood pressure. Proc Natl Acad Sci U S A 2007; 104: 5449-5454.

Wennerholm UB, Henningsen AK, Romundstad LB, Bergh C, Pinborg A, Skjaerven R, Forman J, Gissler M, Nygren KG, Tiitinen A. Perinatal outcomes of children born after frozen-thawed embryo transfer: a Nordic cohort study from the CONARTaS group. Human reproduction 2013;28: 2545-2553.

Woldringh GH, Hendriks JC, van Klingeren J, van Buuren S, Kollee LA, Zielhuis GA, Kremer JA. Weight of in vitro fertilization and intracytoplasmic sperm injection singletons in early childhood. Fertil Steril 2011;95: 2775-2777.

Woo JG. Fast, Slow, High, and Low: Infant and Childhood Growth as Predictors of Cardiometabolic Outcomes. J Pediatr 2017;186: 14-16.

Wunder D, Ballabeni P, Roth-Kleiner M, Primi MP, Senn A, Chanson A, Germond M, Leyvraz C. Effect of embryo culture media on birthweight and length in singleton term infants after IVF-ICSI. Swiss medical weekly 2014;144: w14038.

http://www.openepi.com.

Yeung EH, Sundaram R, Bell EM, Druschel C, Kus C, Xie Y, Buck Louis GM. Infertility treatment and children's longitudinal growth between birth and 3 years of age. Hum Reprod 2016;31: 1621-1628.

Yin TL, Zhang Y, Li SJ, Zhao M, Ding JL, Xu WM, Yang J. Culture media influenced laboratory outcomes but not neonatal birth weight in assisted reproductive technology. J Huazhong Univ Sci Technolog Med Sci 2015;35: 932-937.

Young LE, Fernandes K, McEvoy TG, Butterwith SC, Gutierrez CG, Carolan C, Broadbent PJ, Robinson J, Wilmut I, Sinclair KD. Epigenetic change in IGF2R is associated with fetal overgrowth after sheep embryo culture. Nature genetics 2001;27: 153-154.

Zander DL, Thompson JG, Lane M. Perturbations in mouse embryo development and viability caused by ammonium are more severe after exposure at the cleavage stages. Biol Reprod 2006;74: 288-294. 
Zandstra H, Brentjens L, Spauwen B, Touwslager RNH, Bons JAP, Mulder AL, Smits LJM, van der Hoeven M, van Golde RJT, Evers JLH et al. Association of culture medium with growth, weight and cardiovascular development of IVF children at the age of 9 years. Hum Reprod 2018.

Zandstra H, Van Montfoort AP, Dumoulin JC. Does the type of culture medium used influence birthweight of children born after IVF? Hum Reprod 2015;30: 2693.

Zegers-Hochschild F, Masoli D, Schwarze JE, laconelli A, Borges E, Pacheco IM. Reproductive performance in oocyte donors and their recipients: comparative analysis from implantation to birth and lactation. Fertility and sterility 2010;93: 2210-2215.

Zhan QT, Pan PP, Xu XR, Lou HY, Lou YY, Jin F. An overview of studies on psychological well-being in children born following assisted reproductive technologies. J Zhejiang Univ Sci B 2013;14: 947-960.

Zhu J, Li M, Chen L, Liu P, Qiao J. The protein source in embryo culture media influences birthweight: a comparative study between G1 v5 and G1-PLUS v5. Human reproduction 2014;29: 1387-1392.

Zhu J, Lin S, Li M, Chen L, Lian Y, Liu P, Qiao J. Effect of in vitro culture period on birthweight of singleton newborns. Hum Reprod 2014;29: 448-454.

Ziebe S, Loft A, Povlsen BB, Erb K, Agerholm I, Aasted M, Gabrielsen A, Hnida C, Zobel DP, Munding B et al. A randomized clinical trial to evaluate the effect of granulocyte-macrophage colony-stimulating factor (GM-CSF) in embryo culture medium for in vitro fertilization. Fertility and sterility 2013;99: 1600-1609. 


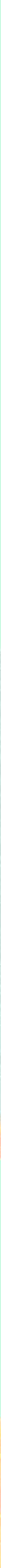




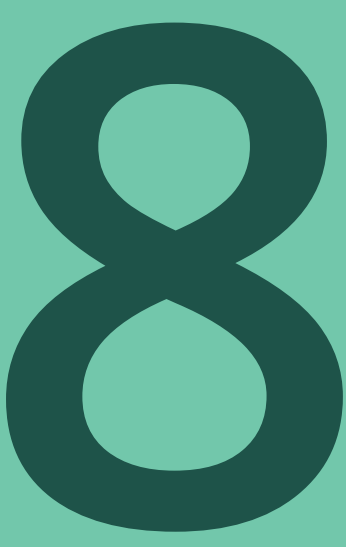

\section{Valorisation}

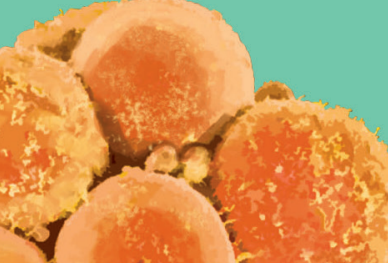





\section{VALORISATION}

In this valorisation paragraph the potential societal impact of the research presented in this thesis will be discussed. This thesis presents the results of a follow-up study in 9-year-old children born after IVF. During an IVF treatment, embryos are cultured in culture medium that may contain a variety of different ingredients. We showed in a previous study that this culture medium can affect the birthweight of children born after IVF. It is also known that birthweight is an important parameter predicting future health risks, and therefore it is important to perform structured follow-up. In this thesis, we present the results of such a follow-up study. We showed that the group of children who were heavier at birth, were on average still heavier and had a higher BMI at 9 years of age if compared to the other group. Cardiovascular development and cognitive development were comparable. Our study was the first to show that culture medium can have an effect on postnatal development.

\section{Relevance}

Since the first successful IVF treatment in 1978, the use of this treatment has increased significantly worldwide. In 2018, it was reported that already more than 8 million babies conceived after IVF/ICSI treatments were born. Research focusing on long-term health and development of the IVF offspring is still scarce. However, there is increasing evidence that pregnancies after ART are associated with a higher risk of adverse outcomes such as low birthweight and also that children born after ART show different outcomes with possible long-term health risks. We have shown that culture medium can affect not only birthweight, but also postnatal weight and growth patterns. Lower birthweight and accelerated postnatal growth are known to be associated with a higher risk for cardiovascular diseases in later life, which underlines the importance of structured and long-term follow up of the children born after IVF.

Embryo culture media are an essential part of the IVF treatment and all cells within the embryo (and thus, indirectly, also all cells within the childs' body) are exposed to the culture medium. At present, in contrast to the drugs used in IVF, there is no uniform regulation or statutory oversight of the composition of embryo culture media. In Europe, the majority of media carry the CE mark, but non-CE media can still be used. Overall, fertility centers choose the media they want to use according to their own preferences. Manufacturers differ on whether and how they give information on the composition of the culture media and the scientific rationale. They are allowed to change their composition without informing their end-users (embryologists) about the changes. In the EU covenant of 2009 it was agreed that the EU would regulate ART culture media and consider them medical devices (Class III). According to the EU medical device regulations, manufacturers are supposed 
to perform structured and long-term follow-up of the offspring who as an embryo were cultured in their culture media, however this is not performed. It is worrisome that the exact quantitative composition is unknown, because this limits the option to correlate specific culture medium ingredients to IVF outcome. The strong financial aspect of this field has led to a lack of transparency regarding composition, which does not facilitate the scientific effort to refine IVF culture media and reach a consensus on which one to use in which circumstances.

\section{Target groups}

The results of this thesis are interesting mostly for IVF clinicians, embryologists and embryo culture medium manufacturers. In the future, it could also be potentially useful for health economists, insurance companies and politicians. The results are only to a limited extent interesting for couples that are currently undergoing fertility treatment or already have a child conceived via IVF. Since it is still very unclear to what extent culture media can influence outcome, and moreover, which culture medium (ingredient) would be better, it is too early to inform patients about this. Patients should be informed that the general health outcome of children born after IVF is reassuring, but that all techniques that are applied during the embryonic phase are possibly capable of creating an increase in risks in later life.

\section{Activities/Innovations}

All chapters in this thesis have been or will be published in high-ranked scientific journals. Also, we have discussed our findings at numerous national and international conferences to increase awareness and attention for this important topic. Also the findings were discussed in popular scientific journals and newspapers (New Scientist and Daily Mail) that are easy accessible to patients and other people interested in IVF. We are currently performing a new multicenter randomized controlled trial to compare pregnancy and perinatal outcome between a sequential culture medium and a single-step culture medium to increase the knowledge and corroborate the evidence on their effects.

The European Society of Human Reproduction and Embryology (ESHRE) has appointed a working group on culture media with members from the special interest groups Embryology, Safety and Quality in ART, and Genetics. This working group encourages constructive co-operations with IVF culture media manufacturers regarding transparency, composition and quality control parameters. Also, in 2016 the Oxford University Press published a press release in Human Reproduction from the working group that received worldwide attention. The results of our study will be presented to this working group and will add valuable information and therefore enforce the working groups' position in the lobby with manufacturers to become transparent on the composition of their culture media. 
It is very important to acknowledge the possibility that IVF culture media composition, other culture conditions and techniques applied during the IVF treatment can affect postnatal development of the offspring. However, the extent of the adverse outcome is still uncertain and large basic research studies and randomized clinical studies are essential to investigate the aetiology of the adverse outcome.

\section{Schedule and implementation}

Introduction of new techniques, changes in culture media composition and laboratory protocols should be preceded by thorough and structured research upon introduction into daily practice. In reproductive medicine not infrequently a treatment forces itself upon the practitioner long before a suitable theory of the mechanism of action has been developed. Too often, new techniques are introduced into daily practice after just some appealing findings in a few small, underpowered, mostly observational studies. Well-designed and properly powered research is indispensible for improving the techniques used in IVF. With regard to culture media development, changes should be made to the existing regulatory system to achieve transparency and improve monitoring of outcomes to the long-term benefit of ART children. At the moment, only basic quality control and a proof-of-principle from mouse embryo testing is necessary before a new culture medium can be applied in the human IVF clinic. This is not enough to safeguard the health of future IVF children.

An important step forward would be that researchers are allowed to culture embryos just for research purposes. Animal models are in many ways a very good first step for research in the field of reproductive medicine, but the results might not always translate well to the human situation. The most important advantage of animal research is the shorter lifespan of for example mice, with a timespan of a couple of months "Iong-term" effects of culture medium can be investigated. However, humans have a fecundity that is not at all comparable to that of mice and sheep and also there are differences in the nutritional requirements of the embryos. Therefore animal models should not be the only way to gain more insight in the (patho)physiology and to test effectiveness of new techniques, but it should also be performed in human embryos. Unfortunately in The Netherlands, it is not allowed to culture embryos for research and in the latest political coalition agreement, in October 2017, the new Government announced that this will not be allowed for at least the next 4 years. This is a great set-back for research in the field of ART and it should be carefully reconsidered. In comparison to culture media for mouse embryos, media for human embryos are not truly optimized and probably will never be without proper research in human embryos. In order to optimize basic culture parameters for mice, to optimize mouse embryo culture medium, several thousand mouse embryos were used. To achieve similar experiments in humans, the number 
of human embryos would be very high and numerous ethical issues would be raised. However the consequence of this is the introduction of embryo culture media without proper testing in the human.

Challenges involved in the field of reproductive medicine

Although ART has changed the life of many people in a positive way, it is also an increasingly commercial industry. Couples who are trying to conceive are willing to try anything to achieve their biggest wish, i.e. becoming parents. The field of ART is rapidly changing and improving, but many new technologies and methods have been introduced in clinical practice without appropriate evidence to show that the procedure is beneficial to the patient and moreover that the benefits outweigh its potential harms. The biggest difference with other fields in medicine is that fertility patients attend clinics with a "wish" instead of a "complaint". Their attitude is therefore quite different from patients who attend their doctor for example for high blood pressure. Infertility patients are often well informed, either by reading on the internet but also by taking in a lot of (not always correct) advice from their friends and family. Professionals involved in IVF treatments should inform their patients carefully and the main objective should be to be honest about the (im)possibility and usefulness of adding extra techniques. Shared decision making together with well-informed couples is one of the challenges of IVF clinicians.

We hope that the results described in this thesis will increase awareness for the existing legislation and most importantly more strict regulations concerning the clinical implementation of culture media. More stringent regulation on the production of IVF culture media and more flexible legislation on embryo research is required to keep moving forward with the best interest of the patients and their offspring in mind. It is the duty of healthcare professionals and researchers to focus on unravelling the aetiology of the observed differences to be able to improve the techniques in order to reduce the potential lifetime health risks of the IVF offspring. 



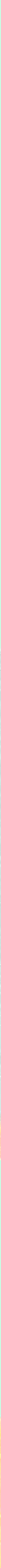




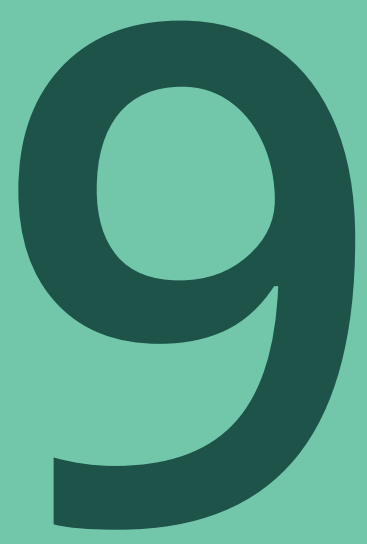

Summary

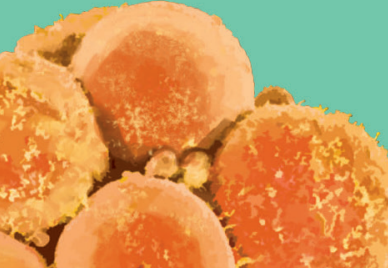





\section{SUMMARY}

Chapter 1 provides a general introduction, as well as an outline of this thesis. It is known that children born after an IVF treatment are at increased risk for adverse perinatal outcome such as low birthweight (LBW) and preterm birth (PTB) compared with naturally conceived children. The etiology behind the observed differences is still to be elucidated. Several possible explanations have been proposed such as differences in patient characteristics, effects induced as a result of the ovarian hyperstimulation or several technical factors involved with the handeling and culture of the gametes and embryos necessary for the IVF treatment. In animal studies, it has been repeatedly observed that changes in the culture medium can cause perinatal and postnatal differences affecting long-term health of the offspring. Evidence from human studies is not yet as convincing, which can be partially explained by the many challenges that are involved in research with effects of culture medium on postnatal development of the offspring. In 2010, we published the results of a study where we compared outcome of children born after embryo culture in two different commercially available culture media. Surprisingly the children born as a result of IVF culture in Vitrolife G1, version 3 (Göteborg, Sweden) culture medium were significantly heavier at birth than the children born after embryo culture in K-SICM (Cook Medical, Brisbane Australie) culture medium. From the Barker hypothesis and the DOHaD hypothesis, it is known that low birthweight is a precursor of an increased risk for (cardiac) diseases in adult life. This thesis focuses on the effects of culture medium on birthweight and on long-term postnatal development of the children born after an IVF/ICSI treatment.

In Chapter 2 we performed a structured literature review on the relationship between culture medium in human IVF and birthweight published before October $15^{\text {th }} 2014$. We included a total of 11 studies that were relevant for the research question. Conventional meta-analysis was not possible since almost all studies compared different culture media. This makes it impossible to provide one answer since all comparisons are different. In the 11 papers, a total of 22 comparisons were done with 19 different culture media couples. Five of the included studies found significant differences in birthweight when offspring born after culture in different culture media were compared and the remaining studies did not find any differences in birthweight. All studies had several methodological issues, such as a retrospective design in most studies, small sample sizes in the randomized or alternating studies and often a comparison of culture media that were used over a consecutive period of time. Also, the exact composition of the culture media is not publically accessible. As a result, although in both animal and human studies there is increasing evidence that culture medium can affect perinatal outcome like birthweight, the actual effect of culture medium on growth of IVF offspring is still not 
definitively elucidated. More research might also have to focus on effects of different ingredients of the culture media, on long-term effects of culture medium on health and epigenetic (de)regulation of IVF offspring.

In Chapter 3, 4 and 5 we discuss the results of our prospective observational cohort study (MEDIUM-KIDS). To assess how the children from our first culture medium study are developing we performed a prospective observational cohort study. In this study, parents of all singletons from our first culture medium study published in 2010 were approached, to participate in a follow-up study (MEDIUM-KIDS) after the ninth birthday of their child. It concerned all singletons born after an IVF treatment between July 2003-December 2006, when two culture media (either G1 version 3 (Vitrolife AB, Goteborg, Sweden) or K-SICM (Cook Medical, Brisbane, Australia)) were used strictly alternating in our laboratory. A total of 136 children (75 Vitrolife and 61 Cook) were included in the analysis. All follow-up measurements were performed between March 2014 and December 2016.

In Chapter 3, we evaluated the weight and body composition of these children at 9 years of age and compared the two culture medium groups mutually. Height and height corrected for age and gender (SDS scores) were similar in both groups. Weight and BMI of the Cook children was significantly lower than the average weight and BMI of the Vitrolife children. After correction for confounders, the difference in weight and BMI attributable to culture medium was $1.58 \mathrm{~kg}[95 \% \mathrm{Cl} 0.01$ to 3.14$]$ and $0.84 \mathrm{~kg} / \mathrm{m}^{2}[95 \% \mathrm{Cl}$ 0.02 to 1.67 ] respectively with the Vitrolife children being heavier. Furthermore, waist circumference was significantly higher in the Vitrolife group with a corrected difference of $3.21 \mathrm{~cm}$ [95\% Cl 0.60 to 5.81 ] leading to a 0.03 increase [95\% Cl 0.01 to 0.05 ] in waist/ hip ratio. Subscapular skinfolds combined with suprailiacal skinfolds, (defined as truncal adiposity) was also significantly higher in Vitrolife children (adjusted difference $3.44 \mathrm{~cm}$ [95\% Cl 0.27 to 6.62$])$.

This study showed that in vitro culture medium used for human embryos still affects body weight, BMI, truncal adiposity, waist circumference and waist/hip ratio even at the age of 9. These differences may predispose to future (cardiovascular) health risks.

In Chapter 4, the impact of culture medium on the cardiovascular development of the children at 9 years of age was investigated. We compared several markers of (early) cardiovascular dysfunction (such as bloodpressure and endothelial function) and took a blood sample from most children after an overnight fast. For the blood samples, we also included a control group using residual blood stored in our hospitals laboratory from children who had their blood tested for several benign reasons not influencing 
the outcome parameters of our study. Both systolic (adj. beta 0.364 [95\% Cl -2.129 to 2.856]) and diastolic (adj. beta 0.275 [95\% Cl -2.105 to 2.654]) blood pressures ( $\mathrm{mmHg}$ ) were comparable for the two groups. After an overnight fast, TSH, cholesterol, glucose, insulin, LDL, HDL and triglycerides were normal and similar between the two groups. Comparison with the control group showed that HDL was significantly lower in the control group and the triglycerides were significantly higher in the control group compared to the IVF/ICSI groups. Endothelial function in the microcirculation was compared using skin laser-Doppler with iontophoresis by using maximum perfusion units corrected for the baseline value as a measure for vasodilatory capacity. There were no significant differences between the two groups. Cortisol and cortisone concentration in hair samples were also comparable between the two groups. We showed reassuring results on the cardiovascular development of the children in our cohort at this moment. However, as weight and body composition do differ between the groups it is important that long-term health will be closely monitored as cardiovascular alterations might emerge at later age.

In Chapter $\mathbf{5}$ we analysed the cognitive development of the children from the two culture medium groups at 9 years of age. To investigate and compare their cognitive development, we used the Dutch pupil monitoring system called CITO (Dutch Central Institute for Test Development). This monitoring system involves nationwide independent, standardized, academic achievement score-tests to monitor the child's school performance twice a year at fixed time points from $3^{\text {rd }}$ grade onwards. The tests include language skills (vocabulary and orthography), math and reading capability and comprehension. Results from the tests performed between $3^{\text {rd }}$ and $6^{\text {th }}$ grade, expressed as ability scores, were obtained from the school the child went to. To investigate school performance development over the years, we used a mixed effects multilevel model. All analyses showed comparable results for the two culture medium groups. No significant differences were observed for any of the cognitive development domains. Parental level of education was higher in the IVF group if compared to the national average level of education, which most likely explains the higher CITO scores for the IVF children if compared to the National ability scores. Although further research on long-term academic skills and also on behavior is essential, these results are reassuring and should make parents of children born after IVF feel comfortable with their children's cognitive development.

In Chapter 6 we performed a multivariable linear regression analysis to analyse whether growth velocity in early childhood is associated with cardiovascular outcome in the IVF children from the two culture medium groups at 9 years of age. Growth in multiple growth windows were positively associated with blood pressure for example growth from 2-6 years: $B=4.13, P=0.005$. Maximum skin perfusion after acetylcholine was negatively associated with height-adjusted weight gain from 2-6 years: $B=-0.09$ (log scale), $P=0.03$. Also 
several growth windows were positively linked with total adiposity. Lipids, glucose tolerance indices and cortisone were not related to growth. In conclusion, neonatal, infant and childhood growth are positively related to blood pressure in IVF/ ICSI offspring. Growth in childhood was negatively associated with endothelial function. For reasons of generalizability, we only included healthy children, born at term in this study.

Chapter 7 presents a general discussion of the findings addressed in this thesis within the context of the recent literature. Also a short conclusion, wider implications and future prospects are given. 



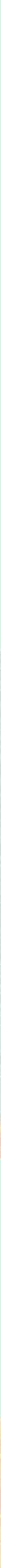



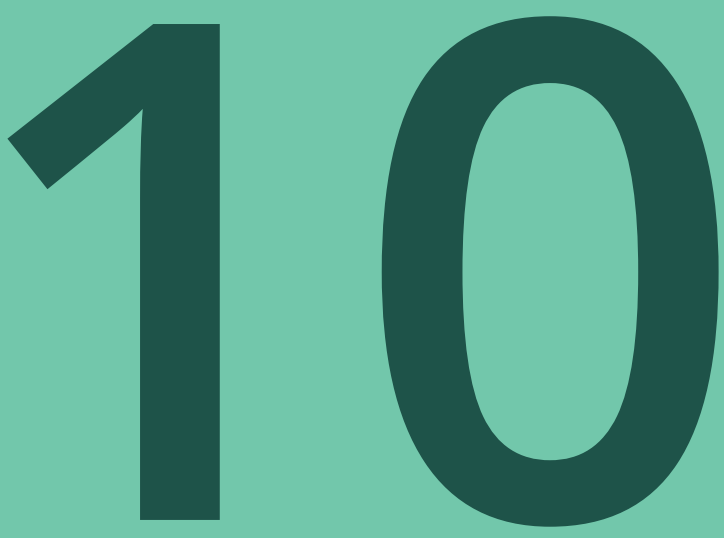

Samenvatting 



\section{SAMENVATTING}

Hoofdstuk 1 geeft een algemene introductie van het onderwerp van dit proefschrift en daarnaast een kort overzicht van de inhoud van dit proefschrift. Het is bekend dat vrouwen die zwanger zijn geworden na een IVF behandeling een hoger risico hebben op complicaties tijdens de zwangerschap zoals een vroeggeboorte of pre-eclampsie. Daarnaast hebben kinderen die geboren zijn na een IVF zwangerschap een verhoogd risico op een lager geboortegewicht vergeleken met kinderen die geboren zijn na natuurlijke bevruchting. Het is nog niet duidelijk wat precies de verklaring is voor de geobserveerde verschillen. Als mogelijke verklaring wordt gedacht aan verschillen in patiëntkenmerken die gepaard gaan met de subfertiliteit, een effect van de ovariële hyperstimulatie of factoren die gepaard gaan met de technische aspecten van de IVF/ICSI behandeling. In dierstudies is het al meermaals aangetoond dat geboortegewicht en postnatale ontwikkeling beïnvloed kunnen worden door het kweekmedium dat gebruikt wordt tijdens de embryokweek. In humane studies is deze relatie nog niet zo duidelijk aangetoond. Dit kan gedeeltelijk verklaard worden door de vele uitdagingen die gepaard gaan met het doen van wetenschappelijk onderzoek naar embryokweek bij humane embryo's. In 2010 publiceerde onze groep de resultaten van een studie waarbij retrospectief de resultaten werden geanalyseerd van een periode waarin we twee verschillende commercieel verkrijgbare kweekmedia alternerend gebruikten. Het bleek dat kinderen die geboren waren na embryokweek in Vitrolife G1, versie 3 (Göteborg, Sweden) kweekmedium een significant hoger geboortegewicht hadden dan de kinderen die als embryo gekweekt waren in K-SICM kweekmedium (Cook Medical, Brisbane Australie). Studies die leidden tot de Barker-hypothese en later de DOHaD-hypothese hebben laten zien dat kinderen die geboren worden met een lager geboortegewicht op latere leeftijd een hoger risico hebben op onder andere cardiovasculaire ziekten. Dit proefschrift focust op het effect van kweekmedium op postnatale ontwikkeling van kinderen die geboren zijn na IVF om te onderzoeken wat de langere termijn gevolgen zijn van het verschil in geboortegewicht.

In Hoofdstuk 2 bespreken we de resultaten van een gestructureerde literatuurstudie over het effect van kweekmedium op geboortegewicht van de kinderen die geboren worden na een IVF/ICSI behandeling. Alle literatuur die gepubliceerd werd voor 15 oktober 2014 werd opgenomen in dit review, waarmee we in totaal 11 studies konden includeren voor de analyse. Het was niet mogelijk om een conventionele meta-analyse te verrichten, omdat bijna alle studies andere kweekmedia vergeleken. In totaal waren er 22 vergelijkingen met 19 verschillende mediakoppels. Vijf van de geïncludeerde studies rapporteerden een significant verschil in geboortegewicht tussen kinderen die als embryo gekweekt waren in verschillende kweekmedia, maar de resterende 6 studies rapporteerden geen verschil in geboortegewicht. Alle studies die geïncludeerd werden 
in de studie hadden methodologische tekortkomingen zoals een retrospectief studie design. Daarnaast vergeleken veel studies kweekmedia die ze in opeenvolgende periodes gebruikt hadden en de studies die de kweekmedia gerandomiseerd (of alternerend) vergeleken, hadden relatief kleine patiëntaantallen. Daarnaast wordt de exacte samenstelling van de verschillende kweekmedia niet openbaar gemaakt door de fabrikanten omdat dit beschouwd wordt als commercieel geheim. Hoewel er dus uit dierstudies en humane studies veel aanwijzingen zijn dat kweekmedium een effect kan hebben op de perinatale uitkomst zoals geboortegewicht, is er nog te weinig overtuigend bewijs in humane studies om dit definitief te kunnen concluderen. Er is nog meer onderzoek nodig naar deze relatie bij humane embryo's. Hierbij zouden ook de effecten van specifieke ingrediënten van de kweekmedia op de lange termijn gezondheid van de IVF kinderen moeten worden meegenomen.

In Hoofdstuk 3, 4 en 5 bespreken we de resultaten van onze prospectieve cohortstudie (MEDIUM-KIDS) die uitgevoerd werd tussen maart 2014 en december 2016. Het doel van deze studie was om te onderzoeken hoe de kinderen uit onze eerste kweekmedium studie (waarbij een verschil in geboortegewicht gevonden werd) zich ontwikkelen. Alle eenlingen die geboren waren na een IVF/ICSI behandeling tussen juli 2003 en december 2006 kwamen in aanmerking voor deelname. In deze periode werd in ons laboratorium gebruik gemaakt van 2 verschillende kweekvloeistoffen (Vitrolife G1, versie 3 (Göteborg, Sweden) en K-SICM (Cook Medical, Brisbane Australie)) op een strikt alternerende manier. Alle ouders van de kinderen die in aanmerking kwamen werden na de ge verjaardag van hun kind benaderd voor deelname aan het onderzoek. In totaal namen 136 kinderen (75 Vitrolife en 61 Cook) deel aan het onderzoek.

Hoofdstuk 3 vergelijkt de antropometrische resultaten van de kinderen uit de twee kweekmedium groepen met betrekking tot hun groei, gewicht en vetgehalte op 9-jarige leeftijd. Lengte en lengte gecorrigeerd voor leeftijd en geslacht was vergelijkbaar tussen de twee groepen. Echter, het gemiddelde gewicht en BMI van de Cook kinderen was significant lager dan het gemiddelde gewicht en BMI van de Vitrolife kinderen. Na correctie voor confounders, was het verschil in gewicht en BMI dat toe te schrijven is aan het kweekmedium respectievelijk $1.58 \mathrm{~kg}$ [95\% Cl 0.01 - 3.14] en $0.84 \mathrm{~kg} / \mathrm{m}^{2}[95 \% \mathrm{Cl} 0.02$ - 1.67]. Daarnaast was de taille-omvang significant hoger in de Vitrolife groep, met een gecorrigeerd verschil van $3.21 \mathrm{~cm}$ [95\%Cl 0.60 - 5.81]. Dit leidde tot een toename van 0.03 [95\% Cl $0.01-0.05$ ] in taille/heup ratio. Deze studie laat zien dat kweekmedium dat gebruikt wordt voor in vitro kweek van embryo's nog altijd effect heeft op het gewicht en BMI van de kinderen op 9 jarige leeftijd. Deze verschillen zouden op latere leeftijd kunnen leiden tot hogere (cardiovasculaire) risico's. 
In Hoofdstuk 4 worden de cardiovasculaire resultaten van de kinderen uit de MEDIUM-KIDS studie besproken. Om te onderzoeken of kweekmedium op 9-jarige leeftijd al een effect zou kunnen hebben op de cardiovasculaire ontwikkeling van de kinderen hebben we meerdere cardiovasculaire parameters onderzocht, zoals bloeddruk en endotheelfunctie. Daarnaast hebben we nuchter bloed afgenomen bij de meeste kinderen. De resultaten van de bloedmonsters hebben we daarnaast ook nog vergeleken met een controlegroep van kinderen die in ons ziekenhuis bloed hadden geprikt voor andere redenen die geen invloed hadden op de waarden waarnaar wij gekeken hebben. De systolische bloeddruk (adj. beta 0.364 [95\%Cl-2.129 - 2.856]) en diastolische bloeddruk (adj. beta 0.275 [95\%Cl -2.105 - 2.654]) (mmHg) waren vergelijkbaar voor de twee groepen. In de bloedmonsters werd gekeken naar TSH, cholesterol, glucose, insuline, LDL, HDL en triglycerides. Deze waarden waren binnen de normaalwaarden en vergelijkbaar voor de twee groepen. Bij de vergelijking met de controlegroep bleek dat HDL significant hoger was in de IVF groepen en dat triglyceriden significant hoger waren in de controlegroep. Endotheelfunctie in de microcirculatie (een vroege marker voor cardiovasculaire dysfunctie) werd geanalyseerd met laser-doppler en iontophorese. Er werd gekeken naar maximale perfusie eenheden en dit was niet verschillend tussen de twee kweekmedium groepen. Cortisol en cortison concentraties in de haarsamples waren niet verschillend tussen de twee groepen. Deze studie laat zien dat de cardiovasculaire ontwikkeling van de kinderen uit ons cohort op 9 jaar normaal is en vergelijkbaar tussen de twee kweekmedium groepen. Het is echter wel belangrijk om de kinderen op langere termijn te vervolgen, zeker omdat gebleken is dat de kinderen in de Vitrolife groep zwaarder waren en een hoger BMI hadden.

In Hoofdstuk 5 beschrijven we de cognitieve ontwikkeling van de kinderen uit de twee kweekmedium groepen. Om dit te onderzoeken hebben we gebruik gemaakt van het CITO leerling volgsysteem. Dit Nederlandse volgsysteem is ontwikkeld om de leerprestaties van leerlingen over de tijd te kunnen volgen. Er wordt gekeken naar taalvaardigheid (woordenschat en spelling), rekenen, begrijpend lezen en technisch lezen. We hebben alle beschikbare resultaten van de kinderen meegenomen van groep 3 tot en met groep 6. Daarbij is gekeken naar de 'vaardigheidsscore' die als een gestandaardiseerde maat kan worden gezien waarmee de kinderen onderling maar ook met een landelijk gemiddelde kunnen worden vergeleken. Om de ontwikkeling over de tijd te kunnen vergelijken hebben we gebruik gemaakt van een mixed effects multilevel model. Alle analyses van de verschillende domeinen lieten zien dat de cognitieve ontwikkeling van de twee kweekmedium groepen vergelijkbaar was. Er waren geen significante verschillen tussen de twee groepen. Als de IVF/ICSI kinderen echter vergeleken werden met de Nederlandse gemiddelden dan scoorde de IVF groep op bijna alle domeinen significant beter. Dit is meest waarschijnlijk (gedeeltelijk) toe te schrijven aan een gemiddeld hoger opleidingsniveau van de IVF ouders 
vergeleken met het gemiddelde nationale opleidingsniveau in dezelfde leeftijdscategorie. De resultaten van deze studie zijn geruststellend met betrekking tot de cognitieve ontwikkeling van de IVF kinderen.

Hoofdstuk 6 beschrijft een multivariabele lineaire regressie analyse om te ontdekken of groeisnelheid op de vroege kinderleeftijd geassocieerd is met de cardiovasculaire uitkomsten op 9-jarige leeftijd van de IVF kinderen uit de eerder genoemde prospectieve cohortstudie. Het bleek dat groeisnelheid in meerdere groei-windows positief geassocieerd was met de bloeddruk, bijvoorbeeld groei van 2-6 jaar: $B=4.13, P=0.005$. Maximale doorbloeding van de microvascularisatie in de huid na toediening van acetylcholine was juist negatief geassocieerd met een snelle gewichtstoename tussen 2-6 jaar ( $B=0.09$ (log scale), $P=0.03$ ). Groeisnelheid in meerdere groei windows waren positief gecorreleerd met het totale vetgehalte. Lipidenprofiel, glucose tolerantie en cortison concentraties waren niet gerelateerd aan groeisnelheid. Concluderend, neonatale groei en groei in de vroege jeugd waren positief gecorreleerd met bloeddruk in kinderen die geboren zijn na IVF/ ICSI. Groei in de vroege jeugd was negatief geassocieerd met endotheelfunctie. Om de resultaten van deze studie ook te kunnen extrapoleren naar andere populaties hebben we ervoor gekozen prematuur geboren kinderen te excluderen.

In Hoofdstuk 7 wordt tenslotte een algemene discussie en beschouwing van de resultaten gepresenteerd in de context van de huidige literatuur. Daarnaast wordt er kort ingegaan op de toekomstige implicaties van de resultaten van dit onderzoek. 



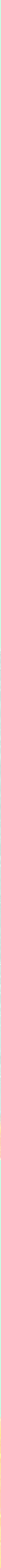



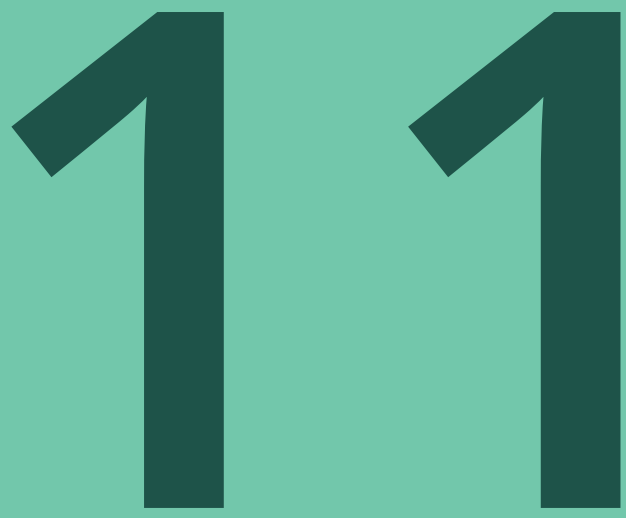

Appendices

Co-authors and their affiliations

List of publications

Dankwoord

Curriculum Vitae 



\section{CO-AUTHORS AND THEIR AFFILIATIONS}

Prof. Dr.JLH Evers, Department of Obstetrics \& Gynaecology, Maastricht University Medical Centre, Maastricht, The Netherlands.

Dr. JCM Dumoulin, IVF department, Maastricht University Medical Centre, Maastricht, The Netherlands

Dr. APA van Montfoort, IVF department, Maastricht University Medical Centre, Maastricht, The Netherlands

LBPM Brentjens, IVF Department, Obstetrics \& Gynaecology, Maastricht University Medical Centre, Maastricht, The Netherlands

B. Spauwen, Department of Obstetrics \& Gynaecology, Maastricht University Medical Centre, Maastricht, The Netherlands

Dr. RNH Touwslager, Department of Pediatric Medicine, Maastricht University Medical Centre, Maastricht, The Netherlands

Dr. JAP Bons, Department of Clinical Chemistry and Laboratory Medicine, Maastricht University Medical Centre, Maastricht, The Netherlands

Dr. AL Mulder, Department of Neonatology, University Hospital Antwerp, Antwerp, Belgium

Dr. LJM Smits, Department of Epidemiology, Faculty of Health, Medicine and Life Sciences of Maastricht University, Maastricht, The Netherlands

Dr. MAHBM van der Hoeven, Department of Pediatric Medicine, Maastricht University Medical Centre, Maastricht, The Netherlands

Dr. RJT van Golde, Department of Obstetrics \& Gynaecology, Maastricht University Medical Centre, Maastricht, The Netherlands

Dr. SMJ van Kuijk, Department of Epidemiology, Faculty of Health, Medicine and Life Sciences of Maastricht University, Maastricht, The Netherlands

Dr. LJ Zimmerman, Department of Pediatrics, GROW School for Oncology and Developmental Biology, Maastricht University Medical Center, Maastricht, The Netherlands 



\section{LIST OF PUBLICATIONS}

Zandstra H., Van Montfoort APA, Dumoulin JCM

Does the type of culture medium used influence birthweight of children born after IVF? Human Reproduction. 2015 March; 30(3):530-42

Zandstra H, Brentjens LBPM, Spauwen B, Touwslager RNH, Bons JAP, Mulder AL, Smits LJM, van der Hoeven MAHBM, van Golde RJT, Evers JLH, Dumoulin JCM, Van Montfoort APA. IVF culture medium still affects weight and body composition of child at age 9 Human Reproduction, 2018 Sep 1;33(9):1645-1656

Zandstra H, Smits LJ, van Kuijk SMJ, van Golde RJT, Evers JLH, Dumoulin JCM, van Montfoort APA.

No effect of culture medium on cognitive development of 9-year-old children Human Reproduction Open, 2018 Sep; Issue 4, pp 1-8

Zandstra $\mathrm{H}$, Van Montfoort AP, Zimmermann LJ, and Touwslager RN Growth and weight trajectories of IVF children compared to naturally conceived children. Submitted September 2018

H. Zandstra, H.J.M.M. Mertens.

Improving external cephalic version for foetal breech presentation.

FW in ObGyn, 2013, 5 (2): 85-90

Nicole M.L. Veldhorst-Janssen, Audrey A.A. Fiddelers, H. Zandstra, Alfons G.H. Kessels, Marco A.E. Marcus, Cees Neef, Paul-Hugo M. van der Kuy Patient satisfaction with intranasal Fentanyl for breakthrough pain. Journal of Palliative Medicine, 2012 Jun;15(6):631-2 


\section{ORAL PRESENTATIONS}

Effect of culture medium on cardiovascular characteristics of 9-year-old IVF/ICSI singletons. ESHRE Annual meeting Geneva. July 2017

Weight and waist circumference of IVF children at the age of 9 years still affected by embryo culture medium.

ESHRE Annual meeting Geneva. July 2017 (Clinical Science Award for best oral presentation.)

Weight and waist circumference of IVF children at the age of 9 years still affected by embryo culture medium.

VFS annual meeting, Antwerp, November 2017

Effect van kweekmedium op de ontwikkeling van kinderen geboren na IVF.

Zuyderland wetenschapssymposium. June 2018 


\section{POSTER PRESENTATIONS}

IVF culture medium still affects growth, weight and body composition of child at age 9 GROW science day. November 2017 and Zuyderland Wetenschapssymposium June 2017.

Significantly more pain during intercourse and impaired sexual functioning in women with endometriosis.

ESHRE annual meeting, Londen. July 2013 



\section{DANKWOORD}

Een promotietraject heeft verrassend genoeg een heel aantal overeenkomsten met het voltooien van een Ironman. Het duurt lang, het is vaak leuk en interessant maar soms ook zwaar. op sommige momenten zie je het even niet meer zitten, maar zolang je niet opgeeft kom je uiteindelijk wel bij de eindstreep. Net zoals je bij het voorbereiden voor een Ironman hulp, begeleiding en gezelschap nodig hebt is dit ook zeker het geval bij een promotietraject. Alleen kom je er echt niet. De eerste periode van het promotietraject zou je kunnen vergelijken met het zwemonderdeel in de triathlon. De mensen op kop weten precies welke richting ze op moeten, ze zien de boeien en zien dus gemakkelijk de beste en snelste route. De beginnende zwemmers daarentegen, moeten al veel moeite doen om hun hoofd boven water te houden en zijn soms de richting even kwijt. De leiders zijn dan onmisbaar om de beginners te laten zien waar ze op de juiste plek uit het water kunnen komen. In de midden- en eindfase van de wedstrijd (en van het promotietraject) helpt het om te zien dat de leiders altijd blijven doorgaan (ondanks tegenslagen) en dat zij de juiste 'lijn' blijven vasthouden tot het eind. Ik ben ontzettend blij dat ik tijdens mijn gehele promotietraject, het geluk heb gehad met zulke fantastische (bege) leiders te hebben mogen samenwerken. Zonder jullie was het me nooit gelukt en had ik ook zeker niet zoveel geleerd.

Prof. Dr. J.L.H. Evers, geachte promotor, beste Prof. Evers. Met uw enthousiasme, altijd extreem snelle reacties en goede inhoudelijke feedback hebt u vooral in de laatste fase van mijn promotietraject een heel belangrijke bijdrage geleverd aan dit proefschrift. Met een record van slechts drie uur reactietijd voor revisies op een compleet artikel (helaas wel op vrijdagavond terwijl ik dacht even lekker weekend te kunnen vieren) kon ik altijd rekenen op inhoudelijk sterke en opbouwende feedback op de manuscripten. Vooral op het eind heeft u mij aangemoedigd "de vaart erin te houden". Ook toen u nog als gynaecoloog werkzaam was heb ik nog enkele jaren het genoegen gehad om met u samen te mogen werken en veel van u te kunnen leren. Ik vind het een eer uw laatste promovenda te zijn en wens $u$ een fijn en ontspannen pensioen zonder deadlines. $U$ zult nu wel iets anders moeten zoeken om uw tijd door te komen.

Dr. A.P.A. van Montfoort, beste Aafke. Mijn altijd gestructureerde rots in de branding! Zonder jouw enorme enthousiasme, kennis, georganiseerdheid en positieve instelling was me dit nooit gelukt! Bedankt dat je mij de kans bood om dit onderzoek samen met jou op te zetten en uit te voeren. Altijd had jij weer een verhelderende en gestructureerde kijk als ik er even niet uitkwam. Je leerde me om gestructureerd te denken ("Maak nou toch eens lijstjes!") en met jou voerde ik altijd interessante inhoudelijke discussies. Je kritische, maar altijd nuttige opmerkingen op de manuscripten maakten dat ik aanvankelijk nog met enige angst mijn email opende als er weer een volledig rood gekleurde verbeterde 
versie terugkwam. Jouw input was echter altijd opbouwend, haarscherp en nuttig. Ik had me echt geen betere copromotor kunnen wensen. Bedankt voor alles wat ik van je heb mogen leren en heel erg veel succes in je toekomstige carrière.

Dr. J.C.M. Dumoulin, beste John. De rustige en altijd weloverwogen tegenhanger van Aafke. Bedankt voor de bijdrage die je geleverd hebt aan dit proefschrift. Natuurlijk eerst aan de Mediumstudies die vooraf gingen aan dit onderzoek, maar ook bij de resultaten van de MEDIUM-KIDS studie was je altijd betrokken. Het was een eer om met deze onderzoekslijn verder te mogen gaan. Bedankt ook voor alles wat ik naast het onderzoek over embryologie en IVF van je geleerd heb in de kliniek. Over de vorm van de manuscripten werd nog wel eens wat gediscussieerd ("Zou je dat niet wat voorzichtiger kunnen omschrijven?"), maar jij maakte dit dreamteam helemaal compleet.

Geachte leden van de leescommissie: prof. dr. M.E.A. Spaanderman, prof. dr. S. Repping, prof. dr. C.D. Dirksen, prof. dr. D. Brison en dr. W.J.M. Gerver: bedankt voor het lezen en beoordelen van dit proefschrift

Een ander onmisbaar onderdeel van de triathlon is het "materiaal". Zoals je niet kunt fietsen zonder fiets, kun je ook geen onderzoek doen zonder proefpersonen.

Grote dank aan alle ouders van de kinderen die de moeite hebben genomen om deel te nemen aan het onderzoek. Jullie trotseerden belangeloos de (toen nog) lange files bij de Geusselt en namen vrij van het werk om een bijdrage te leveren aan dit onderzoek. Het was mooi en vaak indrukwekkend om jullie verhalen te horen over hoe jullie de IVF-behandeling destijds beleefd hadden. Hoewel jullie je geen leven meer zonder kind konden voorstellen was niemand vergeten hoeveel impact de onzekere IVF-periode destijds had gehad. Het was een ontzettend waardevolle toevoeging op mijn klinische werk als IVF-arts. Veel dank ben ik natuurlijk ook verschuldigd aan de kids die mee hebben gedaan aan dit onderzoek. Jullie hadden allemaal zo je eigen motieven om mee te doen. De een omdat ze zelf ook dokter wilde worden, de ander deed het voor een dagje vrij van school of voor het beloofde cadeautje van ons. Ik was aangenaam verrast dat de meesten van jullie het uiteindelijk aandurfden om bloed te laten prikken ondanks dat jullie dat vooraf allemaal eng vonden. Jullie ontwapenende vragen maakten elke meetochtend weer leuk en het is toch onverwachte winst dat ik nu ook alle liedjes van 'Frozen' en 'Verschrikkelijke Ikke' letterlijk mee kan zingen. 
Het fietsonderdeel is het grootste en langste onderdeel van de triathlon. Zeker in de Ironman lijkt er soms geen eind aan te komen. Op sommige momenten doen je benen zoveel pijn dat je denkt dat je niet meer kunt. Wat is het dan fijn als de mede-fietsers om je heen je af en toe even bemoedigend toespreken. Ook tijdens de trainingen is het heerlijk om met gelijkgestemden samen te rijden en om van meer ervaren triatleten slimme trucs kunt leren om beter te worden. Tijdens het promotieonderzoek is de "middenfase" waarin je de metingen uitvoert ook een erg belangrijke fase. Je komt obstakels tegen op de weg waar je overheen moet en ook hier heb ik het genoegen gehad met heel veel mensen samen te mogen werken die me tips en trucs leerden, bemoedigend toespraken en me hielpen deze obstakels te overwinnen.

Brenda. Als researchnurse én moeder van twee IVF kinderen heb jij een dubbele bijdrage geleverd aan de MEDIUM-KIDS studie. Met Lara heb je meegedaan aan de studie, maar daarnaast deed je de metingen als ik ze niet kon doen vanwege de IVF-poli. Heel erg bedankt voor al je hulp en handige tips voor het overwinnen van de CTCM- en METC-bureaucratie.

Veronica, Eva, Eri en Eefje. Mijn Glycocheck maatjes. Bedankt voor de flexibiliteit en prettige samenwerking bij het delen van de GlycoCheck en voor het delen van frustraties over dit ingewikkelde systeem.

Suhreta. Hoewel het vormen en overdragen van de grote GGD-database tot ons beider frustratie erg lang duurde is het ons uiteindelijk toch gelukt om deze waardevolle database met groeigegevens van ruim 20.000 Limburgse controlekinderen toe te voegen aan ons databestand. Bedankt voor je hulp en vasthoudendheid. Helaas heeft het artikel over deze database dit proefschrift niet gehaald, maar uiteindelijk gaat het er zeker komen!

Ewka en Sander. Mijn voorgangers op de MEDIUM-studies onderzoekslijn. Jullie hebben de basis gelegd voor deze onderzoekslijn en ik heb veel gehad aan de data die jullie tijdens jullie promotieonderzoek verzameld hebben.

Agnes Scheffers en Ivo Thijssen bedankt voor jullie hulp bij het interpreteren van de CITO testscores die toch ingewikkelder bleken te zijn dan we dachten.

Dank aan alle Sponsoren: Vitrolife, Origio, Goodlife, Merck, Ferring en Birr die financieel hebben bijgedragen aan de druk van dit proefschrift. 
Collega IVF-artsen: Guusje en Dees, jullie hebben me ingewerkt en wegwijs gemaakt in de wondere wereld van de IVF. Naast jullie verschillende benaderingen en persoonlijkheden kreeg ik de ruimte om mijn eigen stijl te ontwikkelen. Je kunt je eerste stappen als nieuwbakken dokter maar 1 keer zetten en ik ben heel blij dat ik die mede dankzij jullie in alle rust heb kunnen zetten. Bedankt voor de altijd fijne samenwerking, de vele kopjes koffie samen en het spuien over werk- en onderzoekfrustraties. Het was heel fijn om dit samen met jullie te kunnen delen. Het is extra bijzonder dat we elkaar nu weer tegenkomen in de opleiding. Elena, hoewel we dachten een timide meisje binnen te hebben gehaald, bleek je (gelukkig!) al gauw een pittige tante die wist waar ze voor stond. Je bent een goede dokter en met jouw vastberadenheid gaat jouw proefschrift en die opleidingsplaats er zeker ook komen. Inger, mijn opvolgster, ik heb je leren kennen als een lieve en betrokken dokter. Je bent in het afgelopen jaar gegroeid in je rol als dokter en ik weet zeker dat je een grote aanwinst bent voor de afdeling en de patiënten.

Alle IVF-verpleegkundigen en doktersassistenten met wie ik samen heb mogen werken: Carla, Tonny, Germaine, Katinka, Erna, Mahasin, Danielle, Silvia, Lilian, Elena, Cecile, Marlie en Sandra. Ik zal de gezellig lunches, de goede sfeer en de fijne samenwerking met jullie nooit vergeten! Altijd in voor een praatje, altijd geïnteresseerd in mij als persoon en mijn rare sportieve uitspattingen die jullie nooit snapten (“Wanneer ga je nou eens uitrusten op vakantie?!"). We deelden lief en leed. Pas later heb ik me gerealiseerd hoe speciaal het samenwerken in zo'n klein, hecht team is. De verhuizing van de afdeling die we samen hebben neergezet heeft voor veel stress gezorgd, maar we kunnen trots zijn op wat er nu staat, het is prachtig geworden!

Janneke, Ron, Olivier en dr. Dunselman. Bedankt voor de jarenlange fijne samenwerking op de IVF-afdeling en voor alles wat ik van jullie heb mogen leren. Ik kijk ernaar uit om straks ook op het gebied van de algemene gynaecologie en de verloskunde weer van jullie te kunnen leren. Ron, jouw altijd ontnuchterende manier om naar problemen te kijken was soms frustrerend (als ik het probleem weer eens veel groter vond dan jij) maar meestal ook lekker relativerend. Jouw tips voor het voeren van een goed gesprek over moeilijke onderwerpen komen nog vaak van pas ("Leun om te beginnen eens achterover in plaats van bijna bij de mensen op schoot te kruipen en probeer eens langer dan 1 seconde je mond te houden.") Het blijft verrassend wat voor leuke wending in het gesprek dit soms kan opleveren. Olivier, jouw "randstedelijke directheid" toen je binnenkwam op onze afdeling was soms even schrikken, maar meestal heerlijk verfrissend. Bedankt voor alles wat ik van je geleerd heb, je frisse blik en de inhoudelijke discussies die ik met je kon voeren. Janneke, jouw betrokkenheid bij de afdeling en patiëntenzorg is ongekend. Jouw stiptheid en enorme hoeveelheid kennis is iets dat ik hoop ooit een klein beetje te mogen hebben. Bedankt voor alles wat je ook naast het werk voor mij gedaan hebt en 
ook voor je lieve aanbod om de laatste versie van mijn proefschrift te checken op typ- en schoonheidsfoutjes. Je bent echt een topper.

Alle collega's van het IVF-laboratorium: Edith C, Josien, Inge, Myriam, Sabina, Marion, Marijke, Edith L, Marij, Danielle bedankt voor de fijne samenwerking tijdens mijn tijd als IVF-arts.

Bob, het stuk dat we samen hebben geschreven is een heel waardevolle toevoeging aan mijn proefschrift geworden en stond binnen recordtijd op papier dankzij jouw inspanningen. Ik ben je echt eeuwig dankbaar! Bedankt ook voor je hulp bij het opstarten van de iontoforese metingen in het begin van de studie en voor je inhoudelijke bijdrage later tijdens het schrijven. Naast jou voelde ik me altijd een beetje dom door jouw enorme hoeveelheid parate kennis en je zorgvuldigheid.

Prof. Feron, bedankt voor uw hulp met het verzamelen van de GGD groeicurves van alle kinderen. De afspraken met $u$ duurden altijd langer dan gepland en uw altijd goede humeur werkte aanstekelijk. $U$ dacht altijd alleen maar in mogelijkheden i.p.v. problemen en dat was heerlijk en verfrissend.

Dr. Mertens, bedankt dat u mij enthousiast hebt gemaakt voor het vak van gynaecoloog en ook voor het doen van onderzoek. Tevens dank voor de kans die u mij geboden heeft om als ANIOS te komen werken in het Zuyderland waarna ik mee kon solliciteren voor de opleiding.

Gaby, bedankt dat jouw deur altijd open stond en nog steeds staat voor een luchtig praatje (bijvoorbeeld over onze katten), maar ook voor alle minder luchtige praatjes waarvoor ik altijd bij jou terecht kon. Bedankt voor je steun de afgelopen jaren en ook voor je super lieve aanbod om mijn dankwoord te checken, omdat het meest gelezen hoofdstuk van dit boekje natuurlijk grammaticaal gewoon perfect moet zijn.

Maatschap Gynaecologie \& Obstetrie van het Zuyderland ziekenhuis. Bedankt voor de ruimte en de steun die ik van jullie heb gekregen om dit proefschrift zoveel mogelijk af te kunnen ronden voordat ik zou starten met de opleiding. Doordat ik drie maanden vrij kon nemen voordat ik startte met de opleiding, kon ik de belangrijkste stappen in de afronding van dit proefschrift zetten. Bedankt ook voor de fijne opleidingssfeer waarbinnen ik mijn eerste stappen heb kunnen zetten in mijn opleiding tot gynaecoloog. Ik kijk ernaar uit nog veel meer van jullie te kunnen leren. Zeker nu ik me volledig op de opleiding kan richten zonder de tijd te hoeven verdelen tussen het proefschrift en de opleiding. 
Collega arts-assistenten en klinisch verloskundigen van het Zuyderland ziekenhuis. Bedankt voor jullie stoomcursus verloskunde toen ik in maart 2017 startte als groentje op de verloskamers. Het was een behoorlijke overgang van de IVF waarvan ik voor mijn gevoel "alles" wist, naar de verloskamers waar ik "helemaal niks" wist. Ik heb in korte tijd heel veel van jullie geleerd en kreeg van jullie het vertrouwen dat ik het uiteindelijk allemaal wel onder de knie zou krijgen. Bedankt voor het aanhoren van mijn geklaag af en toe in de afrondende fase van dit proefschrift en bedankt voor de fijne samenwerking elke dag weer opnieuw. Op naar nog heel veel mooie en leerzame jaren samenwerking én gezelligheid op en buiten het werk!

Als het fietsonderdeel achter de rug is, dan is het tijd voor het laatste onderdeel: het lopen. Tijdens het looponderdeel staan de fans en supporters al langs de kant aan te moedigen en de finish met het thuisfront is in zicht. Wat gaf het een fijn gevoel om te weten dat er tijdens deze hele periode zoveel lieve mensen langs de kant stonden die mij aanmoedigden.

Lieve vriendinnen van Rowzee en EJD2007. Hoewel we elkaar tegenwoordig vaak langere tijd niet zien is het altijd weer fijn om samen te zijn en bij te kunnen praten. Stiekem is er toch wel veel veranderd sinds we elkaar leerden kennen als eerstejaars studentjes. Wat is het leuk om te zien hoe iedereen zich heeft ontwikkeld. In het bijzonder Marlies, Nathalie en Sofie, bedankt voor de onvoorwaardelijke vriendschap en dat jullie er altijd waren en zijn. Echte vriendschap is onbetaalbaar.

Lieve vriendinnen van de Sushi-Club. Samenwerken met jullie maakt het werk nog leuker dan het al is. Bedankt voor jullie steun, heerlijke humor en voor de vele gezellige en relaxte avondjes bijpraten en hard lachen onder het genot van de beste sushi die er bestaat.

Farah en Ilja. Onze tijd samen in Zuid-Afrika heeft de basis gelegd voor een mooie vriendschap. Ondanks de afstand en de drukke agenda's blijven we toch af en toe tijd vinden om elkaar weer te zien én stonden jullie bij mijn Ironman finish met het mooiste spandoek langs de kant.

Carcia, Kim en Vero. Wat begon als trainingsmaatjes bij de triathlonvereniging groeide uit tot een mooie en waardevolle vriendschap, waarbij samen trainen al lang niet meer het belangrijkste is. Nu dit proefschrift klaar is komt er snel weer tijd voor samen taartjes eten en koffie drinken, bijpraten onder het hardlopen en die barista-workshop. 
Lieve Linda, wat begon als een slimme en gezellige WESP student op het MEDIUM-KIDS onderzoek, groeide uit tot een goede vriendschap en later tot mijn grote blijdschap ook collega IVF-arts. Tijdens jouw stage periode op de MEDIUM-KIDS studie heb jij mij enorm geholpen met dit onderzoek. Samen konden we ook nadat je al als IVF arts werkte discussiëren over de resultaten en je was net zo enthousiast als ik toen het gewichtsverschil significant bleek te zijn. Je bent een lieve en kundige dokter, was een super collega, maar bovenal ben ik blij dat je zo'n goede vriendin geworden bent. Ik ben blij dat jij vandaag mijn paranimf wilde zijn!

Lieve "50's", Danne en Eef. Zonder jullie was het leven naast het werk zoveel minder leuk geweest. Onze altijd avontuurlijke vakanties, weekendjes weg en trainingsstages, vele avondjes lachen en praten en ontelbare avondrondjes samen op de racefiets zijn onbetaalbaar. Op naar nog heel veel meer mooie herinneringen samen! Danne, ondanks dat we de afgelopen twee jaar samenwerkten, sportten, vakanties, etentjes en weekendjes weg deden én naast elkaar woonden, zijn we elkaar nog steeds niet zat. Al vaker stonden we samen voor hete vuren en kreeg jij mij rustig en zelfverzekerd als ik weer eens zenuwachtig was voor de start van een wedstrijd. Ik ben heel blij dat jij vandaag naast mij staat als paranimf.

Lieve Suus, we zijn al zo lang vriendinnen dat ik niet beter weet dan dat jij er altijd bent. Hoewel jouw promotieonderzoek in een totaal andere hoek zit konden we toch af en toe lekker wat onderzoek-frustraties delen doordat onze trajecten gelijk liepen. Wat fijn dat ik in september de kunst even heb kunnen afkijken bij jouw verdediging! Ik kijk uit naar nog heel veel mooie weekendjes weg en fijne avonden wijndrinken samen.

Lieve Judith, lief "klein" zusje. Hoewel we vroeger door het leeftijdsverschil wat minder samen de(el)den, is dat verschil nu we allemaal volwassen zijn helemaal niet meer merkbaar. Ik vind het mooi om te zien wat een mooie, zelfstandige en volwassen vrouw je geworden bent. Doordat jij me hielp met de DNA extracties in het lab waren deze lange en saaie dagen een stuk beter te verdragen. Mooi om te zien hoe enthousiast jij kon worden van het lab-werk dat ik alleen maar ontzettend saai vond.

Lieve Dorien, mijn lieve tweelingzus. Al ons hele leven bewandelen we nagenoeg hetzelfde pad en ik zou oprecht niet weten wat ik zonder jou zou moeten. Hoewel we vast van plan waren niet óók nog hetzelfde specialisme te kiezen is dit toch niet gelukt. Het is mooi om nu ook de opleiding tot gynaecoloog samen te kunnen delen. Deze promotie is zo ongeveer het enige dat we anders gedaan hebben in ons leven. Wie weet komt die gynaecologische privékliniek met veel continuïteit van zorg er ooit nog wel (" $U$ ziet dag en nacht dezelfde dokter"). 
Papa en mama. Jullie hebben ons altijd gestimuleerd het beste uit onszelf te halen en door te gaan als er even iets tegenzat. Daarnaast leerde ik van jullie om verantwoordelijkheid te nemen en meerdere dingen naast elkaar te doen. Hoewel jullie vonden dat ik daarin de laatste jaren wat overdreven heb (wellicht hebben jullie daar een punt) hebben jullie me altijd gesteund in wat ik doe en weet ik dat ik altijd op jullie kan rekenen. Bedankt daarvoor!

Lieve Vin. Zonder jou is het leven echt een heel stuk minder leuk. Hoewel je wellicht af en toe gedacht moet hebben dat mijn laptop belangrijker was dan jij, ben je me altijd blijven steunen en probeerde je te zorgen dat ik af en toe nog wat anders deed dan typen of werken. Ik kijk uit naar onze toekomst samen, naar nog heel veel slechte woordgrappen, fijne ritjes en loopjes en heerlijke vakanties waarin ik niet eigenlijk nog even wil werken aan dat proefschrift. 




\section{CURRICULUM VITAE}

Heleen Zandstra werd geboren op 24 maart 1988 te Geleen als tweede van een eeneiige tweeling en groeide op in Sittard. In 2006 behaalde zij haar VWO diploma aan de Trevianum Scholengroep te Sittard en verhuisde ze naar Maastricht om daar Geneeskunde te gaan studeren aan de Universiteit van Maastricht. Gedurende haar studie bracht zij driemaal een periode door in het buitenland voor een (keuze)coschap, waaronder een keuze-coschap obstetrie in Oeganda. Het laatste jaar van haar studie was zij als semiarts gynaecologie \& obstetrie werkzaam in het Orbis Medisch Centrum te Sittard waar zij kennis maakte met het doen van wetenschappelijk onderzoek en waar ze definitief besloot zich in deze richting te willen specialiseren. Direct na het behalen van haar artsexamen startte zij als IVF-arts in het Maastricht Universitair Medisch Centrum. Deze functie zou zij gedurende 4.5 jaar combineren met het doen van wetenschappelijk onderzoek dat uiteindelijk leidde tot dit proefschrift. Vanaf maart 2017 was zij werkzaam als ANIOS gynaecologie \& obstetrie in het Zuyderland Medisch Centrum te Heerlen/Sittard en op 1 januari 2018 is zij gestart met de opleiding tot gynaecoloog in ditzelfde ziekenhuis. Heleen woont samen met Vincent Harder in Maastricht. 